\title{
Single-Step Formation of Pyrimido[4,5-d]pyridazines by a Pyrimidine-Tetrazine Tandem Reaction
}

\author{
Juraj Galeta, Michal Šála, Martin Dračínský, Milan Vrábel, Zdeněk Havlas, and \\ Radim Nencka
}

Institute of Organic Chemistry and Biochemistry, Academy of Sciences of the Czech Republic, Flemingovo nám. 2, 16610 Prague, Czech Republic

\section{Table of Contents}

1. General procedures and spectral data (a. Pyrimidines; b. Tetrazines; c. Final products)

2. Side products and intermediates of fluoropyrimidine $\mathbf{S} \mathbf{4}$ with tetrazines

3. Determination of regioselectivity

4. Following the reaction by HPLC-MS and NMR measurements

5. Computational methods and supplemental data

6. NMR Spectra

General Information. Unless otherwise noted, all reagents were purchased from commercial suppliers and used as received. All reactions leading to products 6 were carried out under a dry argon atmosphere. Melting points were determined in open capillaries on a Büchi B-540 apparatus. NMR spectra were recorded at 400 $\mathrm{MHz}\left({ }^{1} \mathrm{H}\right)$ and $100 \mathrm{MHz}\left({ }^{13} \mathrm{C}\right)$ with $\mathrm{CDCl}_{3}$ as solvent. Data are presented as follows: chemical shift (in ppm), multiplicity ( $\mathrm{s}=$ singlet, $\mathrm{bs}=$ broad singlet, $\mathrm{d}=$ doublet, $\mathrm{t}=$ triplet, $\mathrm{q}=$ quartet, sept = septet, $\mathrm{m}=$ multiplet), coupling constant $(\mathrm{J} / \mathrm{Hz})$ and integration. Residual proton $(\delta 7.26 \mathrm{ppm})$ served as an internal standard for ${ }^{1} \mathrm{H}$ $\mathrm{NMR}$ and $\mathrm{CDCl}_{3}$ ( $\delta 77.23 \mathrm{ppm}$ ) for ${ }^{13} \mathrm{C}$ NMR spectra. High-resolution mass spectra (HRMS) were recorded in the positive ESI mode on a LTQ Orbitrap XL (Thermo Fisher Scientific). Column chromatography was performed on Silica gel 60 (Fluka) and thin-layer chromatography (TLC) on Silica gel $60 \mathrm{~F}_{254}$ foils (Merck). HPLC-MS measurements were performed in acetonitrile on Shimadzu HPLC-system coupled to a single quadrupole LC-MS 2020 system equipped with ESI ionization source. The column used was Luna $3 \mu \mathrm{m} \mathrm{C18}(100 \times 4.6 \mathrm{~mm})$ and gradient: $5 \%$ B to $95 \%$ B in 5 min and keeping $95 \%$ B for $7 \mathrm{~min}$. The absorbance was measured at $254 \mathrm{~nm}$. The diffraction data were collected at $120 \mathrm{~K}$ using Rigaku diffractometer, equipped with MicroMax-007HF DW rotating anode $X$-ray source $\left(\lambda=0.71075 \AA\right.$, Mo $K_{\alpha}$ radiation), multilayer optic VariMax DW, partial $\chi$ geometry goniometer, Saturn 724+ HG CCD detector and a Cryostream cooling device. CrystalClear a software was used for data collection. Data reduction was carried out using the CrysAlisPro ${ }^{b}$ software. The crystal was a nonmerohedral twin and the twinning was taken into account during the data reduction (computed twin ratio: 58.6 $\%$ and $41.4 \%$ ). Primary model was obtained from major twin component data by SHELXT $T^{c}$ program and the structure was refined on data from both twin components using $S H E L X L^{c}$ program. All hydrogen atoms were placed to calculated positions (confirmed by maxima in the difference Fourier map) and were refined as riding, with their $U_{\text {iso }}$ values set to $1.2 U_{\text {eq }}$ of parent atom. After the refinement, the twin ratio remains unchanged.

\footnotetext{
${ }^{a}$ Rigaku (2014). CrystalClear-SM Expert 2.1 b32

${ }^{\mathrm{b}}$ Agilent Technologies (2013). CrysAlisPro Software system, Version 1.171.37.35

${ }^{\mathrm{c}}$ Acta Cryst. 2008, A64, 112-122
} 


\section{1) General procedures and spectral data (a. Pyrimidines; b. Tetrazines; $c$. Final products)}

\section{a. Pyrimidines}

General procedure for starting pyrimidines S4-S6 preparation: 2,4-Dichloro-5-halopyrimidine S1-S3 $(3 \mathrm{~g})$ was dissolved in acetonitrile $(70 \mathrm{~mL})$ and $\mathrm{Et}_{3} \mathrm{~N}$ (1.25 eq.) and $\mathrm{EtSH}(1.25$ eq.) were added dropwise. After $1 \mathrm{~h}$ at RT, the mixture was filtered and extracted with AcOEt (3x), dried over $\mathrm{Na}_{2} \mathrm{SO}_{4}$ and filtered. Organics were evaporated and the residue was purified by column chromatography $\left(\mathrm{CHCl}_{3}, \mathrm{SiO}_{2}\right)$.

Representative procedure for preparation of compound S4: 2,4-Dichloro-5-flouropyrimidine S1 (3 g, $18 \mathrm{mmol}$ ) was dissolved in acetonitrile $(70 \mathrm{~mL})$ and $\mathrm{Et}_{3} \mathrm{~N}(3.14 \mathrm{~mL}, 22.5 \mathrm{mmol}, 1.25$ eq.) and $\mathrm{EtSH}$ $(1.67 \mathrm{~mL}, 22.5 \mathrm{mmol}, 1.25$ eq.) were added dropwise. After $1 \mathrm{~h}$ at $\mathrm{RT}$, the mixture was filtered and extracted with AcOEt (3x), dried over $\mathrm{Na}_{2} \mathrm{SO}_{4}$ and filtered. Organics were evaporated and the residue was purified by column chromatography $\left(\mathrm{CHCl}_{3}, \mathrm{SiO}_{2}\right)$.

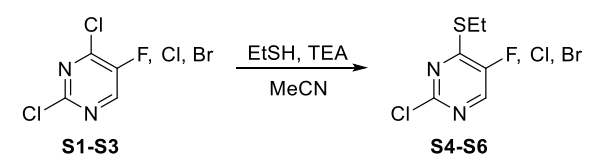

\section{2-Chloro-4-(ethylthio)-5-fluoropyrimidine (S4)}

Yield: $2.77 \mathrm{~g}(80 \%)$ as colorless solid;

${ }^{1} \mathrm{H}$ NMR $\left(400 \mathrm{MHz}, \mathrm{CDCl}_{3}\right): \delta_{\mathrm{H}} 8.06\left(\mathrm{~d},{ }^{3} \mathrm{~F}_{\mathrm{F}, \mathrm{H}}=1.5,1 \mathrm{H}, \mathrm{CH}\right), 3.22\left(\mathrm{q},{ }^{3} \mathrm{~J}_{\mathrm{H}, \mathrm{H}}=7.4,2 \mathrm{H}, \mathrm{CH}_{2}\right), 1.39$ $\left(\mathrm{t},{ }^{3} \mathrm{~J}_{\mathrm{H}, \mathrm{H}}=7.4,3 \mathrm{H}, \mathrm{CH}_{3}\right)$;

$\left.{ }^{13} \mathrm{CNMR}(100 \mathrm{MHz}, \mathrm{CDCl})_{3}\right): \delta_{\mathrm{C}} 162.2\left(\mathrm{~d},{ }^{2} J_{\mathrm{F}, \mathrm{C}}=17.2, \mathrm{C}\right), 154.9\left(\mathrm{~d},{ }^{4} \mathrm{~J}_{\mathrm{F}, \mathrm{C}}=3.6, \mathrm{C}\right), 154.5\left(\mathrm{~d},{ }^{1} J_{\mathrm{F}, \mathrm{C}}=261.5, \mathrm{C}\right)$, $141.9\left(\mathrm{~d},{ }^{2} \mathrm{~J}_{\mathrm{F}, \mathrm{C}}=22.3, \mathrm{CH}\right), 24.0\left(\mathrm{~d},{ }^{4} \mathrm{~J}_{\mathrm{F}, \mathrm{C}}=1.3, \mathrm{CH}_{2}\right), 14.1\left(\mathrm{~s}, \mathrm{CH}_{3}\right)$;

HRMS (EI) m/z calculated for $\mathrm{C}_{6} \mathrm{H}_{6} \mathrm{ClFN}_{2} \mathrm{~S}: 191.9924$, found: 191.9925 .

\section{2,5-Dichloro-4-(ethylthio)pyrimidine (S5)}

Yield: $3.28 \mathrm{~g}(96 \%)$ as colorless solid; ${ }^{1} \mathrm{H} \mathrm{NMR}\left(400 \mathrm{MHz}, \mathrm{CDCl}_{3}\right): \delta_{\mathrm{H}} 8.18(\mathrm{~s}, 1 \mathrm{H}, \mathrm{CH}), 3.20\left(\mathrm{q},{ }^{3} \mathrm{~J}_{\mathrm{H}, \mathrm{H}}=7.4,2 \mathrm{H}, \mathrm{CH}_{2}\right), 1.38\left(\mathrm{t},{ }^{3} \mathrm{~J}_{\mathrm{H}, \mathrm{H}}=\mathrm{Cl}^{\left.7.4,3 \mathrm{H}, \mathrm{CH}_{3}\right) \text {; }}\right.$

${ }^{13} \mathrm{C} \mathrm{NMR}\left(100 \mathrm{MHz}, \mathrm{CDCl}_{3}\right): \delta_{\mathrm{C}} 171.4(\mathrm{C}), 158.0(\mathrm{C}), 154.4(\mathrm{CH}), 127.2(\mathrm{C}), 25.0\left(\mathrm{CH}_{2}\right), 13.8\left(\mathrm{CH}_{3}\right)$;

HRMS (EI) m/z calculated for $\mathrm{C}_{6} \mathrm{H}_{6} \mathrm{Cl}_{2} \mathrm{~N}_{2} \mathrm{~S}: 207.9629$, found: 207.9627 .

\section{5-Bromo-2-chloro-4-(ethylthio)pyrimidine (S6)}

Yield: $3.14 \mathrm{~g}(94 \%)$ as colorless solid (contains $\sim 10 \%$ of disubstituted product);

Major product:

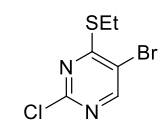

${ }^{1} \mathrm{H}$ NMR $\left(400 \mathrm{MHz}, \mathrm{CDCl}_{3}\right): \delta_{\mathrm{H}} 8.29(\mathrm{~s}, 1 \mathrm{H}, \mathrm{CH}), 3.20\left(\mathrm{q}, 3^{3} \mathrm{~J}_{\mathrm{H}, \mathrm{H}}=7.4,2 \mathrm{H}, \mathrm{CH}_{2}\right), 1.39\left(\mathrm{t}, 3^{3} \mathrm{H}_{\mathrm{H}}=7.4,3 \mathrm{H}\right.$, $\mathrm{CH}_{3}$ );

${ }^{13} \mathrm{CNMR}\left(100 \mathrm{MHz}, \mathrm{CDCl}_{3}\right): \delta_{\mathrm{C}} 172.9$ (C), $158.9(\mathrm{C}), 157.2(\mathrm{CH}), 116.9(\mathrm{C}), 25.7\left(\mathrm{CH}_{2}\right), 13.8\left(\mathrm{CH}_{3}\right)$;

HRMS (EI) m/z calculated for $\mathrm{C}_{6} \mathrm{H}_{6} \mathrm{BrClN}_{2} \mathrm{~S}: 251.9124$, found: 251.9129. 
Pyrimidine S8 preparation: 2,4,6-Trichloropyrimidine S7 (3 g, $16.4 \mathrm{mmol})$ was dissolved in acetonitrile $(50 \mathrm{~mL})$ and $\mathrm{Et}_{3} \mathrm{~N}(2.86 \mathrm{~mL}, 20.5 \mathrm{mmol}, 1.25$ eq.) and $\mathrm{EtSH}$ (1.34 mL, $18.0 \mathrm{mmol}, 1.1$ eq.) were added dropwise at $0{ }^{\circ} \mathrm{C}$. After $10 \mathrm{~min}$, the ice bath was removed and the mixture was left to heat up to $\mathrm{RT}(1 \mathrm{~h})$. Then, $\mathrm{Et}_{2} \mathrm{O}(50 \mathrm{~mL})$ was added and after $10 \mathrm{~min}$ of stirring the salt was filtered. Organics were evaporated and the residue was purified by column chromatography $\left(\mathrm{CHCl}_{3} /\right.$ pentan $1: 1, \mathrm{SiO}_{2}$ ). Surprisingly, we obtained two products in $1: 2$ molar ratio (see below).

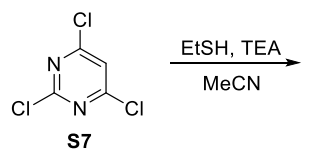

S7

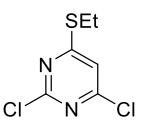

s8

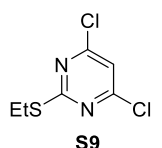

s9

\section{2,4-Dichloro-6-(ethylthio)pyrimidine (S8)}

Yield: $1.95 \mathrm{~g}(57 \%)$ as colorless solid $\left(\mathrm{R}_{\mathrm{f}}=0.44, \mathrm{CHCl}_{3} /\right.$ pentan 1:1);
${ }^{1} \mathrm{H}$ NMR $\left(400 \mathrm{MHz}, \mathrm{CDCl}_{3}\right): \delta_{\mathrm{H}} 7.06(\mathrm{~s}, 1 \mathrm{H}, \mathrm{CH}), 3.16\left(\mathrm{q},{ }^{3} \mathrm{~J}_{\mathrm{H}, \mathrm{H}}=7.4,2 \mathrm{H}, \mathrm{CH}_{2}\right), 1.35\left(\mathrm{t},{ }^{3} \mathrm{~J}_{\mathrm{H}, \mathrm{H}}=\mathrm{Cl}{ }^{\text {SEt }}{ }_{\mathrm{N}}\right.$ 7.4, $\left.3 \mathrm{H}, \mathrm{CH}_{3}\right)$;

${ }^{13} \mathrm{C} \mathrm{NMR}\left(100 \mathrm{MHz}, \mathrm{CDCl}_{3}\right): \delta_{\mathrm{C}} 175.2(\mathrm{~s}, \mathrm{C}), 160.1(\mathrm{~s}, \mathrm{C}), 159.9(\mathrm{~s}, \mathrm{C}), 116.5(\mathrm{~s}, \mathrm{CH}), 24.8\left(\mathrm{~s}, \mathrm{CH}_{2}\right), 14.0$ (s, $\mathrm{CH}_{3}$ );

HRMS (EI) m/z calculated for $\mathrm{C}_{6} \mathrm{H}_{6} \mathrm{Cl}_{2} \mathrm{~N}_{2} \mathrm{~S}: 207.9629$, found: 207.9633 .

\section{4,6-Dichloro-2-(ethylthio)pyrimidine (S9)}

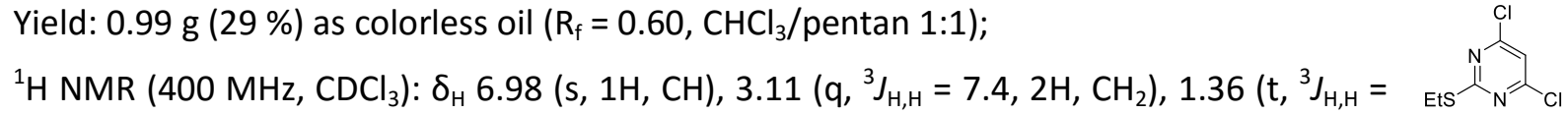
7.4, $\left.3 \mathrm{H}, \mathrm{CH}_{3}\right)$;

${ }^{13} \mathrm{C} \mathrm{NMR}\left(100 \mathrm{MHz}, \mathrm{CDCl}_{3}\right): \delta_{\mathrm{C}} 174.3(\mathrm{~s}, \mathrm{C}), 161.4(\mathrm{~s}, 2 \times \mathrm{C}), 115.8(\mathrm{~s}, \mathrm{CH}), 25.9\left(\mathrm{~s}, \mathrm{CH}_{2}\right), 14.1\left(\mathrm{~s}, \mathrm{CH}_{3}\right)$;

HRMS (EI) m/z calculated for $\mathrm{C}_{6} \mathrm{H}_{6} \mathrm{Cl}_{2} \mathrm{~N}_{2} \mathrm{~S}: 207.9629$, found: 207.9630 . 


\section{b. Tetrazines}

Following tetrazines are known and were prepared according to published procedure - 3,6-bis(3,5dimethyl-1H-pyrazol-1-yl)-1,2,4,5-tetrazine $\quad(\mathbf{S 1 1})^{1}$, 4-(6-chloro-1,2,4,5-tetrazin-3-yl)morpholine $(\mathbf{S 2 5})^{2}$, 3,6-dichloro-1,2,4,5-tetrazine (S28) ${ }^{1}$, 3,6-dimethoxy-1,2,4,5-tetrazine (S29) $)^{3}$, dimethyl 1,2,4,5tetrazine-3,6-dicarboxylate $\quad(\mathbf{S 3 0})^{4}, \quad 3,6$-diisopropyl-1,2,4,5-tetrazine $\quad(\mathbf{S 3 1})^{5}$ and 3,6 -bis(4(trifluoromethyl)phenyl-1,2,4,5-tetrazine (S32) ${ }^{6}$.

\section{3-Isopropyl-6-phenyl-1,2,4,5-tetrazine (S10)}

To a mixture of benzonitrile ( $3 \mathrm{~mL}, 29 \mathrm{mmol})$ and isobutyronitrile $(26.1 \mathrm{~mL}, 290 \mathrm{mmol})$ with hydrazine monohydrate $(64-65 \%$ solution, $14 \mathrm{~mL})$ was carefully added $\mathrm{ZnI}_{2}(0.93$

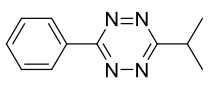
g, $0.29 \mathrm{mmol}$ ) with cooling (cold water). Reaction mixture was then heated to $65^{\circ} \mathrm{C}$ for $24 \mathrm{~h}$, cooled down and evaporated. The residue was suspended in $\mathrm{CH}_{2} \mathrm{Cl}_{2}(40 \mathrm{~mL})$ and solution of sodium nitrite $(5.8 \mathrm{~g})$ in water $(110 \mathrm{~mL})$ was added. The reaction mixture was cooled down to $0{ }^{\circ} \mathrm{C}$ and acetic acid $(4.3 \mathrm{~mL})$ was added dropwise during a period of 30 minutes and stirring was continued at $0{ }^{\circ} \mathrm{C}$ for next $12 \mathrm{~h}$. Organic layer was separated and aqueous layer was extracted with $\mathrm{CH}_{2} \mathrm{Cl}_{2}(2 \times 100 \mathrm{~mL})$. Combined organic phases were dried, evaporated and the product was isolated by column chromatography $\left(\mathrm{SiO}_{2}, \mathrm{CH}_{2} \mathrm{Cl}_{2} /\right.$ pentane 1:2).

Yield: $654 \mathrm{mg}$ (11\%, not optimized) as violet oil;

${ }^{1} \mathrm{H}$ NMR $\left(400 \mathrm{MHz}, \mathrm{CDCl}_{3}\right): \delta_{\mathrm{H}} 8.57-8.64(\mathrm{~m}, 2 \mathrm{H}, \mathrm{Ph}), 7.56-7.65(\mathrm{~m}, 3 \mathrm{H}, \mathrm{PH}), 3.69\left(\mathrm{sept},{ }^{3} \mathrm{~J}_{\mathrm{H}, \mathrm{H}}=6.9\right.$ $\mathrm{Hz}, 1 \mathrm{H}, \mathrm{CH}), 1.57\left(\mathrm{~d},{ }^{3} \mathrm{~J}_{\mathrm{H}, \mathrm{H}}=7.0 \mathrm{~Hz}, 6 \mathrm{H}, 2 \times \mathrm{CH}_{3}\right)$;

${ }^{13} \mathrm{C} \mathrm{NMR}\left(100 \mathrm{MHz}, \mathrm{CDCl}_{3}\right): \delta_{\mathrm{C}} 173.9$ (C), 164.5 (C), $132.7(\mathrm{CH}), 132.1(\mathrm{C}), 129.4(2 \times \mathrm{CH}), 128.1(2 \times \mathrm{CH})$, $34.5(\mathrm{CH}), 21.5\left(2 \times \mathrm{CH}_{3}\right)$;

HRMS (ESI) $\mathrm{m} / \mathrm{z}$ calculated for $\mathrm{C}_{11} \mathrm{H}_{13} \mathrm{~N}_{4}(\mathrm{M}+\mathrm{H})^{+}:$: 201.1135, found: 201.1134 .

\section{Synthesis of tetrazines S12-S27}

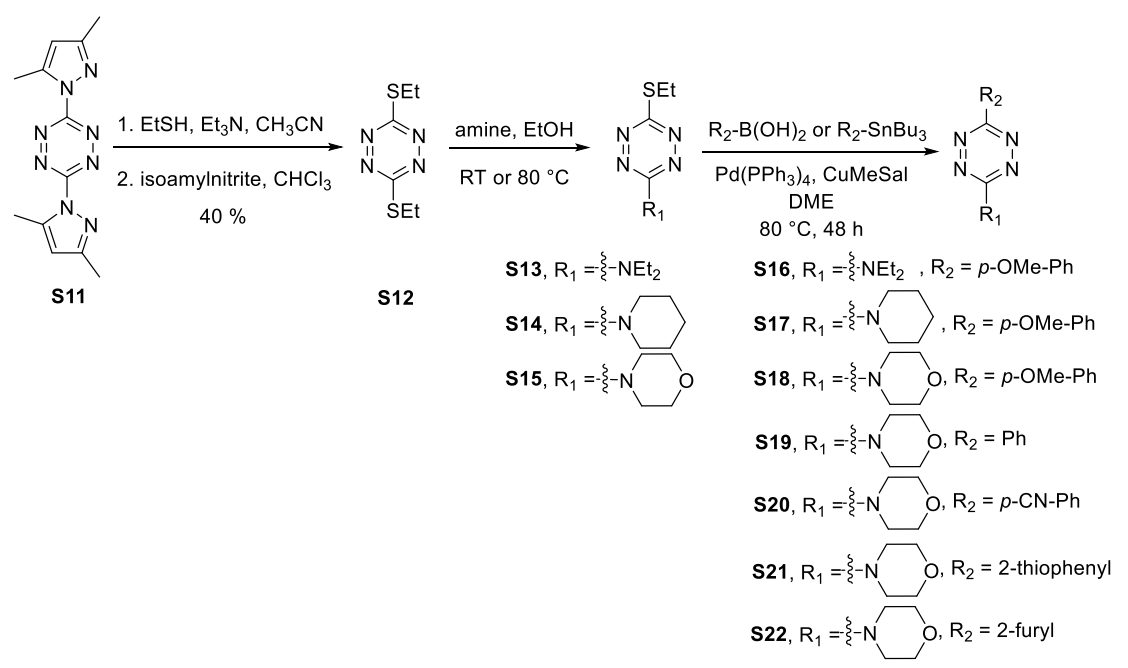

\section{3,6-Bis(ethylthio)-1,2,4,5-tetrazine (S12)}

To a solution of S11 $(3 \mathrm{~g}, 11.1 \mathrm{mmol})$ in acetonitrile $(100 \mathrm{~mL})$ was added $\mathrm{Et}_{3} \mathrm{~N}\left(3.9 \mathrm{~mL}, \quad \mathrm{Ets}-\widehat{N}_{\mathrm{N}-\mathrm{N}}^{\mathrm{N}=\mathrm{N}}-\mathrm{SEt}\right.$
$27.8 \mathrm{mmol})$ followed by EtSH $(2 \mathrm{~mL}, 27.8 \mathrm{mmol})$ and the reaction mixture was stirred for $30 \mathrm{~min}$ at RT and evaporated. Residue was co-evaporated with chloroform $(2 \times 50 \mathrm{~mL})$ and redissolved in chloroform $(50 \mathrm{~mL})$. Isoamylnitrite $(2 \mathrm{~mL})$ was added at RT and the reaction mixture was 
stirred for $1 \mathrm{~h}$. Volatiles were evaporated and product was purified by column chromatography $\left(\mathrm{SiO}_{2}\right.$, $\mathrm{CHCl}_{3}$ ).

Yield: $909 \mathrm{mg}$ (40\%) as red solid;

${ }^{1} \mathrm{H} \mathrm{NMR}\left(400 \mathrm{MHz}, \mathrm{CDCl}_{3}\right): \delta_{\mathrm{H}} 3.30\left(\mathrm{q},{ }^{3} \mathrm{~J}_{\mathrm{H}, \mathrm{H}}=7.3,4 \mathrm{H}, \mathrm{CH}_{2}\right), 1.47\left(\mathrm{t},{ }^{3} \mathrm{~J}_{\mathrm{H}, \mathrm{H}}=7.3,6 \mathrm{H}, \mathrm{CH}_{3}\right)$;

${ }^{13} \mathrm{C} \mathrm{NMR}\left(100 \mathrm{MHz}, \mathrm{CDCl}_{3}\right): \delta_{\mathrm{C}} 172.7$ (C), $25.1\left(\mathrm{CH}_{2}\right), 14.2\left(\mathrm{CH}_{3}\right)$;

HRMS (EI) m/z calculated for $\mathrm{C}_{6} \mathrm{H}_{10} \mathrm{~N}_{4} \mathrm{~S}_{2}(\mathrm{M})^{+}:$202.0347, found: 202.0349.

General procedure for products S13, S14 and S15 formation: Compound S12 (1 g, $4.95 \mathrm{mmol}$ ) was dissolved in ethanol $(16 \mathrm{~mL}$ ) and appropriate amine was added (1.5 eq.) at RT. The reaction mixture was stirred either at RT (for diethylamine, $48 \mathrm{~h}$ ) or at $80^{\circ} \mathrm{C}$ (piperidine - $2 \mathrm{~h}$, morpholine $-14 \mathrm{~h}$ ). The mixture was evaporated and products were isolated by column chromatography $\left(\mathrm{SiO}_{2}\right)$.

Representative procedure for preparation of the compound S13: Compound $\mathbf{S 1 2}(1 \mathrm{~g}, 4.95 \mathrm{mmol})$ was dissolved in ethanol $(16 \mathrm{~mL})$ and diethylamine $(0.77 \mathrm{~mL}, 7.43 \mathrm{mmol}, 1.5$ eq.) was added at RT. The reaction mixture was stirred at RT for $48 \mathrm{~h}$. The mixture was evaporated and products were isolated by column chromatography $\left(\mathrm{SiO}_{2}, \mathrm{CH}_{2} \mathrm{Cl}_{2}\right)$.

\section{$N, N$-Diethyl-6-(ethylthio)-1,2,4,5-tetrazin-3-amine (S13)}

Purification: $\mathrm{CH}_{2} \mathrm{Cl}_{2}$;

Yield: $370 \mathrm{mg}(35 \%)$ as red oil;

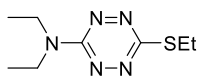

${ }^{1} \mathrm{H}$ NMR $\left(400 \mathrm{MHz}, \mathrm{CDCl}_{3}\right): \delta_{\mathrm{H}} 3.74\left(\mathrm{q},{ }^{3} \mathrm{~J}_{\mathrm{H}, \mathrm{H}}=7.1 \mathrm{~Hz}, 4 \mathrm{H}, \mathrm{NCH}_{2}\right), 3.23\left(\mathrm{q},{ }^{3} J_{\mathrm{H}, \mathrm{H}}=7.3 \mathrm{~Hz}, 2 \mathrm{H}, \mathrm{SCH}_{2}\right), 1.43$ $\left(\mathrm{t},{ }^{3} \mathrm{H}_{\mathrm{H}, \mathrm{H}}=7.3 \mathrm{~Hz}, 3 \mathrm{H}, \mathrm{CH}_{3}\right), 1.26\left(\mathrm{t},{ }^{3} \mathrm{H}_{\mathrm{H}, \mathrm{H}}=7.1 \mathrm{~Hz}, 6 \mathrm{H}, 2 \times \mathrm{CH}_{3}\right)$;

${ }^{13} \mathrm{C} \mathrm{NMR}\left(100 \mathrm{MHz}, \mathrm{CDCl}_{3}\right): \delta_{\mathrm{C}} 164.9$ (C), $159.5(\mathrm{C}), 42.4\left(\mathrm{NCH}_{2}\right), 25.3\left(\mathrm{SCH}_{2}\right), 14.8\left(\mathrm{CH}_{3}\right), 12.8\left(2 \times \mathrm{CH}_{3}\right)$;

HRMS (ESI) $\mathrm{m} / \mathrm{z}$ calculated for $\mathrm{C}_{8} \mathrm{H}_{16} \mathrm{~N}_{5} \mathrm{~S}(\mathrm{M}+\mathrm{H})^{+}: 214.1121$, found: 214.1121 .

\section{3-(Ethylthio)-6-(piperidin-1-yl)-1,2,4,5-tetrazine (S14)}

Purification: $\mathrm{CHCl}_{3}$;

Yield: $903 \mathrm{mg}(81 \%)$ as red solid;

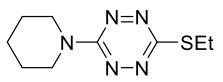

${ }^{1} \mathrm{H}$ NMR $\left(400 \mathrm{MHz}, \mathrm{CDCl}_{3}\right): \delta_{\mathrm{H}} 3.86-3.93\left(\mathrm{~m}, 4 \mathrm{H}, \mathrm{NCH}_{2}\right), 3.23\left(\mathrm{q},{ }^{3} \mathrm{~J}_{\mathrm{H}, \mathrm{H}}=7.3,2 \mathrm{H}, \mathrm{CH}_{2}\right), 1.64-1.78(\mathrm{~m}$, $\left.6 \mathrm{H}, 3 \times \mathrm{CH}_{2}\right), 1.42\left(\mathrm{t},{ }^{3} \mathrm{~J}_{\mathrm{H}, \mathrm{H}}=7.3,3 \mathrm{H}, \mathrm{CH}_{3}\right)$;

${ }^{13} \mathrm{C} \mathrm{NMR}\left(100 \mathrm{MHz}, \mathrm{CDCl}_{3}\right): \delta_{\mathrm{C}} 165.0(\mathrm{C}), 160.2(\mathrm{C}), 44.7\left(\mathrm{NCH}_{2}\right), 25.6\left(2 \times \mathrm{CH}_{2}\right), 25.3\left(\mathrm{CH}_{2}\right), 24.7\left(\mathrm{CH}_{2}\right)$, $14.8\left(\mathrm{CH}_{3}\right)$;

HRMS (EI) $\mathrm{m} / \mathrm{z}$ calculated for $\mathrm{C}_{9} \mathrm{H}_{15} \mathrm{~N}_{5} \mathrm{~S}(\mathrm{M})^{+}: 225.1048$, found: 225.1049 .

\section{4-(6-(Ethylthio)-1,2,4,5-tetrazin-3-yl)morpholine (S15)}

Purification: $\mathrm{AcOEt} / \mathrm{CHCl}_{3}$ 1:10;

Yield: $844 \mathrm{mg}$ (75\%) as red solid;

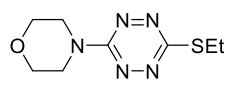

${ }^{1} \mathrm{H}$ NMR $\left(400 \mathrm{MHz}, \mathrm{CDCl}_{3}\right): \delta_{\mathrm{H}} 3.89-3.94\left(\mathrm{~m}, 4 \mathrm{H}, \mathrm{OCH}_{2}\right), 3.81-3.86\left(\mathrm{~m}, 4 \mathrm{H}, \mathrm{NCH}_{2}\right), 3.24\left(\mathrm{q},{ }^{3} \mathrm{~J}_{\mathrm{H}, \mathrm{H}}=7.3\right.$ $\left.\mathrm{Hz}, 2 \mathrm{H}, \mathrm{CH}_{2}\right), 1.43\left(\mathrm{t}^{3} \mathrm{~J}_{\mathrm{H}, \mathrm{H}}=7.3 \mathrm{~Hz}, 3 \mathrm{H}, \mathrm{CH}_{3}\right)$;

${ }^{13} \mathrm{CNMR}\left(100 \mathrm{MHz}, \mathrm{CDCl}_{3}\right): \delta_{\mathrm{C}} 166.5(\mathrm{C}), 160.5(\mathrm{C}), 66.6\left(\mathrm{OCH}_{2}\right), 43.9\left(\mathrm{NCH}_{2}\right), 25.3\left(\mathrm{CH}_{2}\right), 14.7\left(\mathrm{CH}_{3}\right)$; 
HRMS (EI) $\mathrm{m} / \mathrm{z}$ calculated for $\mathrm{C}_{8} \mathrm{H}_{13} \mathrm{~N}_{5} \mathrm{OS}(\mathrm{M})^{+}: 227.0841$, found: 227.0842 .

General procedure for products S16-\$22 preparation, compounds were prepared according to literature : Tetrazine $\mathbf{S 1 3}, \mathbf{S 1 4}$ or $\mathbf{S 1 5}(2.1 \mathrm{mmol})$ and appropriate boronic acid or stannyl derivative (2.5 eq) were mixed with copper(I) 3-methylsalicylate $(992 \mathrm{mg}, 4.6 \mathrm{mmol}$ ) and to this mixture was added dimethoxyethane $(25 \mathrm{~mL})$ and the mixture was three times rinsed with argon. $\mathrm{Pd}\left(\mathrm{PPh}_{3}\right)_{4}(121$ $\mathrm{mg}, 5 \mathrm{~mol} \%$ ) was added and the reaction mixture was heated to $85{ }^{\circ} \mathrm{C}$ and stirred for $48 \mathrm{~h}$. Then, it was cooled down and diluted with saturated aqueous solution of sodium bicarbonate $(100 \mathrm{~mL})$, extracted with dichloromethane $(3 \times 100 \mathrm{~mL})$ and collected organics were dried over $\mathrm{Na}_{2} \mathrm{SO}_{4}$. After filtration and evaporation the residue was purified by column chromatography $\left(\mathrm{SiO}_{2}\right)$.

Representative procedure for preparation of compound S16: Tetrazine S13 (448 mg, $2.1 \mathrm{mmol}$ ) and p-methoxyphenyl boronic acid (798 $\mathrm{mg}, 5.25 \mathrm{mmol}, 2.5 \mathrm{eq})$ were mixed with copper(I) 3methylsalicylate $(992 \mathrm{mg}, 4.6 \mathrm{mmol})$ and to this mixture dimethoxyethane $(25 \mathrm{~mL})$ was added and the mixture was three times rinsed with argon. $\mathrm{Pd}\left(\mathrm{PPh}_{3}\right)_{4}(121 \mathrm{mg}, 5 \mathrm{~mol} \%)$ was added and the reaction mixture was heated to $85^{\circ} \mathrm{C}$ and stirred for $48 \mathrm{~h}$. Then, it was cooled down and diluted with saturated aqueous solution of sodium bicarbonate $(100 \mathrm{~mL})$, extracted with dichloromethane $(3 \times 100$ $\mathrm{mL}$ ) and collected organics were dried over $\mathrm{Na}_{2} \mathrm{SO}_{4}$. After filtration and evaporation the residue was purified by column chromatography $\left(\mathrm{SiO}_{2}\right)$.

\section{N,N-Diethyl-6-(4-methoxyphenyl)-1,2,4,5-tetrazin-3-amine (S16)}

Purification: 1 . $\mathrm{CHCl}_{3} /$ toluene 10:1; 2. $\mathrm{CHCl}_{3} / \mathrm{AcOEt} 20: 1$;

Yield: $223 \mathrm{mg}$ (41\%) as red solid;

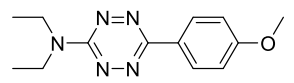

${ }^{1} \mathrm{H} \mathrm{NMR}\left(400 \mathrm{MHz}, \mathrm{CDCl}_{3}\right): \delta_{\mathrm{H}} 8.28-8.37(\mathrm{~m}, 2 \mathrm{H}, 2 \times \mathrm{CH}), 6.99-7.07(\mathrm{~m}, 2 \mathrm{H}, 2 \times \mathrm{CH}), 3.88\left(\mathrm{~s}, 3 \mathrm{H}, \mathrm{OCH}_{3}\right)$, $3.83\left(\mathrm{q},{ }^{3} \mathrm{~J}_{\mathrm{H}, \mathrm{H}}=7.1,4 \mathrm{H}, \mathrm{CH}_{2}\right), 1.42\left(\mathrm{t},{ }^{3} \mathrm{~J}_{\mathrm{H}, \mathrm{H}}=7.1,6 \mathrm{H}, \mathrm{CH}_{3}\right)$;

${ }^{13} \mathrm{C} \mathrm{NMR}\left(100 \mathrm{MHz}, \mathrm{CDCl}_{3}\right): \delta_{\mathrm{C}} 161.7(\mathrm{C}), 159.7$ (C), $158.6(\mathrm{C}), 127.8(2 \times \mathrm{CH}), 125.8(\mathrm{C}), 114.6(2 \times \mathrm{CH})$, $55.6\left(\mathrm{OCH}_{3}\right), 42.5\left(2 \times \mathrm{CH}_{2}\right), 13.0\left(2 \times \mathrm{CH}_{3}\right)$;

HRMS (EI) m/z calculated for $\mathrm{C}_{13} \mathrm{H}_{17} \mathrm{~N}_{5} \mathrm{O}(\mathrm{M})^{+}: 259.1433$, found: 259.1435 .

\section{3-(4-Methoxyphenyl)-6-(piperidin-1-yl)-1,2,4,5-tetrazine (S17)}

Purification: petrolether/AcOEt 10:1;

Yield: $324 \mathrm{mg}(57 \%)$ as red solid;

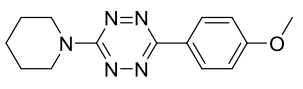

${ }^{1} \mathrm{H}$ NMR $\left(400 \mathrm{MHz}, \mathrm{CDCl}_{3}\right): \delta_{\mathrm{H}} 8.29-8.36(\mathrm{~m}, 2 \mathrm{H}, 2 \times \mathrm{CH}), 7.00-7.07(\mathrm{~m}, 2 \mathrm{H}, 2 \times \mathrm{CH}), 3.97-4.03(\mathrm{~m}$, $\left.4 \mathrm{H}, \mathrm{NCH}_{2}\right), 3.88\left(\mathrm{~s}, 3 \mathrm{H}, \mathrm{OCH}_{3}\right), 1.68-1.83\left(\mathrm{~m}, 6 \mathrm{H}, 3 \times \mathrm{CH}_{2}\right)$;

${ }^{13} \mathrm{C} \mathrm{NMR}\left(100 \mathrm{MHz}, \mathrm{CDCl}_{3}\right): \delta_{\mathrm{C}} 161.7(\mathrm{C}), 160.4(\mathrm{C}), 158.7$ (C), $127.8(2 \times \mathrm{CH}), 125.7(\mathrm{C}), 114.5(2 \times \mathrm{CH})$, $55.6\left(\mathrm{OCH}_{3}\right), 44.7\left(2 \times \mathrm{CH}_{2}\right), 25.7\left(2 \times \mathrm{CH}_{2}\right), 24.7\left(\mathrm{CH}_{2}\right)$;

HRMS (EI) $\mathrm{m} / \mathrm{z}$ calculated for $\mathrm{C}_{14} \mathrm{H}_{17} \mathrm{~N}_{5} \mathrm{O}(\mathrm{M})^{+}: 271.1433$, found: 271.1432 .

\section{4-(6-(4-Methoxyphenyl)-1,2,4,5-tetrazin-3-yl)morpholine (S18)}

Yield: $316 \mathrm{mg}(55 \%)$ as red solid.

All the characteristics match to those previously reported. ${ }^{7}$

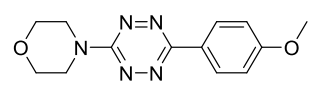




\section{4-(6-Phenyl-1,2,4,5-tetrazin-3-yl)morpholine (S19)}

Purification: Isolation of this compound needed a removal of the starting tetrazine prior to chromatography. The residue after extraction was re-dissolved in

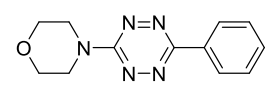
dichloromethane $\left(40 \mathrm{~mL}\right.$ ) and cooled down to $0{ }^{\circ} \mathrm{C}$. To this mixture $m C P B A$ ( $434 \mathrm{mg}, 2.5 \mathrm{mmol}$ ) was added and the mixture was stirred at $0{ }^{\circ} \mathrm{C}$ for $0.5 \mathrm{~h}$. Organic phase was diluted with dichloromethane $(100 \mathrm{~mL})$, washed with saturated solution of sodium bicarbonate $(3 \times 100 \mathrm{~mL})$, dried over $\mathrm{Na}_{2} \mathrm{SO}_{4}$, filtered and evaporated. The residue was purified by column chromatography $\left(\mathrm{SiO}_{2}\right.$, petrolether/AcOEt 4:1).

Yield: $153 \mathrm{mg}(30 \%)$ as red solid;

${ }^{1} \mathrm{H}$ NMR $\left(400 \mathrm{MHz}, \mathrm{CDCl}_{3}\right): \delta_{\mathrm{H}} 8.35-8.45(\mathrm{~m}, 2 \mathrm{H}, \mathrm{Ph}), 7.48-7.59(\mathrm{~m}, 3 \mathrm{H}, \mathrm{Ph}), 4.02-4.10(\mathrm{~m}, 4 \mathrm{H}$, $\left.\mathrm{OCH}_{2}\right), 3.85-3.91\left(\mathrm{~m}, 4 \mathrm{H}, \mathrm{NCH}_{2}\right)$;

${ }^{13} \mathrm{C} \mathrm{NMR}\left(100 \mathrm{MHz}, \mathrm{CDCl}_{3}\right): \delta_{\mathrm{C}} 160.7(\mathrm{C}), 159.7(\mathrm{C}), 132.8(\mathrm{C}), 130.9(\mathrm{CH}), 129.2(2 \times \mathrm{CH}), 126.5(2 \times \mathrm{CH})$, $66.7\left(\mathrm{OCH}_{2}\right), 43.9\left(\mathrm{NCH}_{2}\right)$;

HRMS (EI) $\mathrm{m} / \mathrm{z}$ calculated for $\mathrm{C}_{12} \mathrm{H}_{13} \mathrm{~N}_{5} \mathrm{O}(\mathrm{M})^{+}: 243.1120$, found: 243.1121 .

\section{4-(6-Morpholino-1,2,4,5-tetrazin-3-yl)benzonitrile (S20)}

Purification: 1. petrolether/AcOEt 4:1; 2. petrolether/AcOEt 2:1;

Yield: $254 \mathrm{mg}(45 \%)$ as red solid;

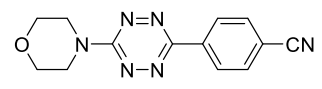

${ }^{1} \mathrm{H}$ NMR $\left(400 \mathrm{MHz}, \mathrm{CDCl}_{3}\right): \delta_{\mathrm{H}} 8.47-8.55(\mathrm{~m}, 2 \mathrm{H}, 2 \times \mathrm{CH}), 7.77-7.85(\mathrm{~m}, 2 \mathrm{H}, 2 \times \mathrm{CH}), 4.05-4.13(\mathrm{~m}$, $\left.4 \mathrm{H}, \mathrm{OCH}_{2}\right), 3.85-3.94\left(\mathrm{~m}, 4 \mathrm{H}, \mathrm{NCH}_{2}\right)$;

${ }^{13} \mathrm{C} \mathrm{NMR}\left(100 \mathrm{MHz}, \mathrm{CDCl}_{3}\right): \delta_{\mathrm{C}} 160.2$ (C), 158.2 (C), $137.0(\mathrm{C}), 133.0(2 \times \mathrm{CH}), 126.7(2 \times \mathrm{CH}), 118.7(\mathrm{C})$, $114.2(\mathrm{C}), 66.6\left(\mathrm{OCH}_{2}\right), 44.0\left(\mathrm{NCH}_{2}\right)$;

HRMS (EI) $\mathrm{m} / \mathrm{z}$ calculated for $\mathrm{C}_{13} \mathrm{H}_{12} \mathrm{~N}_{6} \mathrm{O}(\mathrm{M})^{+}: 268.1073$, found: 268.1074 .

\section{4-(6-(Thiophen-2-yl)-1,2,4,5-tetrazin-3-yl)morpholine (S21)}

2-(Tributylstannyl)thiophene was used as a coupling agent.

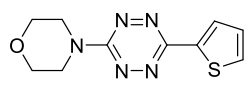

Yield: $225 \mathrm{mg}(43 \%)$ as red solid.

All the characteristics match to those previously reported. ${ }^{7}$

\section{4-(6-(Furan-2-yl)-1,2,4,5-tetrazin-3-yl)morpholine (S22)}

2-(Tributylstannyl)furan was used as a coupling agent.

Purification: 1. petrolether/AcOEt 3:1; 2. toluene/AcOEt 8:1;

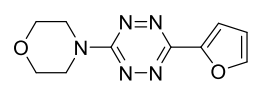

Yield: $203 \mathrm{mg}$ (42\%) as red solid;

${ }^{1} \mathrm{H}$ NMR (400 MHz, CDCl 3 ): $\delta_{\mathrm{H}} 7.67\left(\mathrm{dd},{ }^{3} J_{\mathrm{H}, \mathrm{H}}=1.8,{ }^{4} J_{\mathrm{H}, \mathrm{H}}=0.9 \mathrm{~Hz}, 1 \mathrm{H}, \mathrm{CH}\right), 7.30\left(\mathrm{dd},{ }^{3} J_{\mathrm{H}, \mathrm{H}}=3.4,{ }^{4} J_{\mathrm{H}, \mathrm{H}}=\right.$ $0.9 \mathrm{~Hz}, 1 \mathrm{H}, \mathrm{CH}), 6.61\left(\mathrm{dd},{ }^{3} J_{\mathrm{H}, \mathrm{H}}=3.4,{ }^{4} J_{\mathrm{H}, \mathrm{H}}=1.8 \mathrm{~Hz}, 1 \mathrm{H}, \mathrm{CH}\right), 4.01-4.07\left(\mathrm{~m}, 4 \mathrm{H}, \mathrm{OCH}_{2}\right), 3.84-3.90(\mathrm{~m}$, $4 \mathrm{H}, \mathrm{NCH}_{2}$ );

${ }^{13} \mathrm{C} \mathrm{NMR}\left(100 \mathrm{MHz}, \mathrm{CDCl}_{3}\right): \delta_{\mathrm{C}} 160.1$ (C), 154.7 (C), 148.0 (C), $145.3(\mathrm{CH}), 112.4(\mathrm{CH}), 111.9(\mathrm{CH}), 66.7$ $\left(\mathrm{OCH}_{2}\right), 43.9\left(\mathrm{NCH}_{2}\right)$;

HRMS (EI) $\mathrm{m} / \mathrm{z}$ calculated for $\mathrm{C}_{10} \mathrm{H}_{11} \mathrm{~N}_{5} \mathrm{O}_{2}(\mathrm{M})^{+}:$:233.0913, found: 233.0912 . 


\section{6-Morpholino-1,2,4,5-tetrazin-3-yl benzoate (S24)}

To a solution of $\mathbf{S} 23^{2}$ (955 mg, $3.7 \mathrm{mmol}$ ) in 2-propanol $(12 \mathrm{~mL}$ ) was added in three portions potassium tert-butoxide $(452 \mathrm{mg}, 4 \mathrm{mmol})$ and the reaction mixture was stirred at RT for $1 \mathrm{~h}$. The precipitated salt was filtered and washed with 2-propanol and dried in air. The intermediate was dissolved in water $(7 \mathrm{~mL})$ and acidified to $\mathrm{pH} \approx 3$. The reaction mixture was evaporated to dryness (co-evaporated with ethanol $(2 \times 25 \mathrm{~mL})$ and pyridine $(2 \times 25 \mathrm{~mL}))$. The residue was

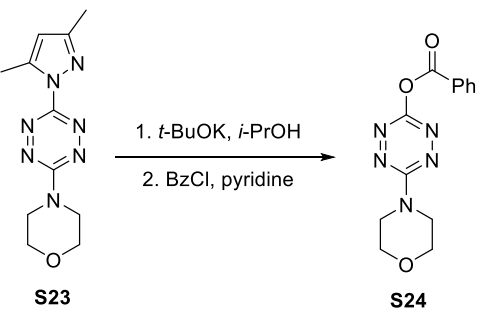
suspended in pyridine $(20 \mathrm{~mL})$ and benzoyl chloride $(0.52 \mathrm{~mL}, 4.5 \mathrm{mmol})$ was added. After an overnight stirring, the reaction was quenched with water $(10 \mathrm{~mL})$ and evaporated. The residue was partitioned between ethylacetate $(200 \mathrm{~mL})$ and water $(100 \mathrm{~mL})$. Organic phase was washed with water $(100 \mathrm{~mL}), 5 \%$ hydrochloric acid $(100 \mathrm{~mL})$, water $(100 \mathrm{~mL})$ and sat. aq. solution of sodium bicarbonate $(2 \times 100 \mathrm{~mL})$, dried over $\mathrm{Na}_{2} \mathrm{SO}_{4}$ and evaporated. The residue was purified by column chromatography $\left(\mathrm{SiO}_{2}\right.$, toluene/AcOEt 20:1).

Yield: $330 \mathrm{mg}(31 \%)$ as orange solid;

${ }^{1} \mathrm{H}$ NMR $\left(400 \mathrm{MHz}, \mathrm{CDCl}_{3}\right): \delta_{\mathrm{H}} 8.24-8.30(\mathrm{~m}, 2 \mathrm{H}, \mathrm{Ph}), 7.67-7.73(\mathrm{~m}, 1 \mathrm{H}, \mathrm{Ph}), 7.52-7.60(\mathrm{~m}, 2 \mathrm{H}, \mathrm{Ph})$, $4.00-4.06\left(\mathrm{~m}, 4 \mathrm{H}, \mathrm{OCH}_{2}\right), 3.86-3.91\left(\mathrm{~m}, 4 \mathrm{H}, \mathrm{NCH}_{2}\right)$;

${ }^{13} \mathrm{C} \mathrm{NMR}\left(100 \mathrm{MHz}, \mathrm{CDCl}_{3}\right): \delta_{\mathrm{C}} 164.4(\mathrm{C}), 161.8(\mathrm{C}), 161.6(\mathrm{C}), 134.8(\mathrm{CH}), 131.0(2 \times \mathrm{CH}), 129.0(2 \times \mathrm{CH})$, $127.8(\mathrm{C}), 66.6\left(\mathrm{OCH}_{2}\right), 44.3\left(\mathrm{NCH}_{2}\right)$;

HRMS (ESI) $\mathrm{m} / \mathrm{z}$ calculated for $\mathrm{C}_{13} \mathrm{H}_{14} \mathrm{~N}_{5} \mathrm{O}_{3}(\mathrm{M}+\mathrm{H})^{+}:$:288.1091, found: 288.1092 .

\section{6-Morpholino-1,2,4,5-tetrazine-3-carbonitrile (S26)}

To a solution of $\mathbf{S 2 5 ^ { 2 }}$ (150 mg, $0.74 \mathrm{mmol}$ ) in DMF ( $3 \mathrm{~mL}$ ) was added KCN $(58 \mathrm{mg}$, $0.89 \mathrm{mmol}$ ) and the reaction mixture was stirred at RT for 2 hours. Then, it was diluted with water $(30 \mathrm{~mL})$ and extracted with $\mathrm{Et}_{2} \mathrm{O}(2 \times 60 \mathrm{~mL})$. Organics were dried over $\mathrm{Na}_{2} \mathrm{SO}_{4}$ and evaporated. The residue was purified by column chromatography $\left(\mathrm{SiO}_{2}\right.$, petrolether/ AcOEt 4:1).

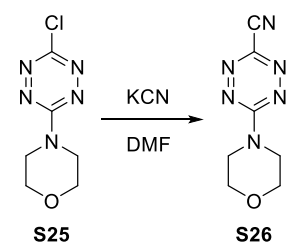

Yield: $107 \mathrm{mg}$ (75\%) as red solid;

${ }^{1} \mathrm{H} N M R\left(400 \mathrm{MHz}, \mathrm{CDCl}_{3}\right): \delta_{\mathrm{H}} 4.11-4.16\left(\mathrm{~m}, 4 \mathrm{H}, \mathrm{OCH}_{2}\right), 3.86-3.91\left(\mathrm{~m}, 4 \mathrm{H}, \mathrm{NCH}_{2}\right)$;

${ }^{13} \mathrm{CNMR}\left(100 \mathrm{MHz}, \mathrm{CDCl}_{3}\right): \delta_{\mathrm{C}} 157.4$ (C), 144.2 (C), 114.0 (C), $66.5\left(\mathrm{OCH}_{2}\right), 44.2\left(\mathrm{NCH}_{2}\right)$;

HRMS (ESI) $\mathrm{m} / \mathrm{z}$ calculated for $\mathrm{C}_{7} \mathrm{H}_{8} \mathrm{~N}_{6} \mathrm{NaO}(\mathrm{M}+\mathrm{Na})^{+}: 215.0652$, found: 215.0653 .

\section{4-(6-(1H-Pyrrol-1-yl)-1,2,4,5-tetrazin-3-yl)morpholine (S27)}

Solution of $\mathbf{S} 25^{2}(250 \mathrm{mg}, 0.91 \mathrm{mmol})$ and pyrrole $(126 \mu \mathrm{L}, 1.8 \mathrm{mmol})$ in dry THF $(15 \mathrm{~mL})$ was cooled down to $0{ }^{\circ} \mathrm{C}$. Sodium hydride $(60 \%$ in mineral oil, $73 \mathrm{mg}$, $1.8 \mathrm{mmol}$ ) was added in three portions at $0{ }^{\circ} \mathrm{C}$ and the reaction mixture was allowed to warm to RT and stirred for $2 \mathrm{~h}$. Then, it was quenched with sat. aq. solution of $\mathrm{NH}_{4} \mathrm{Cl}(20 \mathrm{~mL})$ and extracted with $\mathrm{Et}_{2} \mathrm{O}(2 \times 40 \mathrm{~mL})$. Organic phases were dried over $\mathrm{Na}_{2} \mathrm{SO}_{4}$ and evaporated. The residue was purified by column chromatography $\left(\mathrm{SiO}_{2}\right.$, petrolether/AcOEt 4:1).

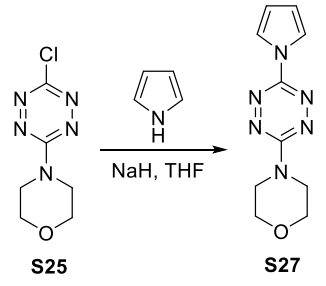

Yield: $243 \mathrm{mg}$ (84\%) as red solid;

${ }^{1} \mathrm{H}$ NMR $\left(400 \mathrm{MHz}, \mathrm{CDCl}_{3}\right): \delta_{\mathrm{H}} 7.71-7.79(\mathrm{~m}, 2 \mathrm{H}, 2 \times \mathrm{CH}), 6.40-6.47(\mathrm{~m}, 2 \mathrm{H}, 2 \times \mathrm{CH}), 3.95-4.01(\mathrm{~m}$, $\left.4 \mathrm{H}, \mathrm{OCH}_{2}\right), 3.85-3.91\left(\mathrm{~m}, 4 \mathrm{H}, \mathrm{NCH}_{2}\right)$; 
${ }^{13} \mathrm{C} \mathrm{NMR}\left(100 \mathrm{MHz}, \mathrm{CDCl}_{3}\right): \delta_{\mathrm{C}} 161.7$ (C), $155.4(\mathrm{C}), 118.3(2 \times \mathrm{CH}), 112.9(2 \times \mathrm{CH}), 66.6\left(\mathrm{OCH}_{2}\right), 44.2$ $\left(\mathrm{NCH}_{2}\right)$;

HRMS (EI) m/z calculated for $\mathrm{C}_{10} \mathrm{H}_{12} \mathrm{~N}_{6} \mathrm{O}(\mathrm{M})^{+}: 232.1073$, found: 232.1074 . 


\section{c. Final Products}

General Procedure for the products 6a-n formation: To the solution of functionalized pyrimidine $(0.55 \mathrm{mmol})$ and substituted tetrazine $(0.5 \mathrm{mmol})$ in anhydrous toluene $(5 \mathrm{~mL})$ under argon atmosphere was added dropwise $0.5 \mathrm{M}$ solution of KHMDS in toluene $(0.6 \mathrm{mmol})$ over a period of 10 min. After $12 \mathrm{~h}$ at $50{ }^{\circ} \mathrm{C}(6 \mathrm{a}-\mathrm{d})$ or RT $(6 \mathrm{e}-\mathrm{n})$, the reaction mixture was quenched with sat. aq. $\mathrm{NH}_{4} \mathrm{Cl}$, extracted with AcOEt $(3 \times)$ and dried over $\mathrm{Na}_{2} \mathrm{SO}_{4}$. After filtration, organics were evaporated and the residue was purified by column chromatography $\left(\mathrm{SiO}_{2}\right)$ and crystallized from acetone.

Representative procedure for preparation of compound 6a: To the solution of 5-bromopyrimidine ( $87 \mathrm{mg}, 0.55 \mathrm{mmol}, 1.1 \mathrm{eq}$ ) and diphenyltetrazine (117 mg, $0.5 \mathrm{mmol}, 1$ eq.) in anhydrous toluene (5 $\mathrm{mL}$ ) under argon atmosphere, $0.5 \mathrm{M}$ solution of KHMDS in toluene $(0.6 \mathrm{mmol}, 1.2$ eq.) was added dropwise over a period of $10 \mathrm{~min}$. After $12 \mathrm{~h}$ at $50{ }^{\circ} \mathrm{C}$, the reaction mixture was quenched with sat. aq. $\mathrm{NH}_{4} \mathrm{Cl}$, extracted with AcOEt $(3 \times)$ and dried over $\mathrm{Na}_{2} \mathrm{SO}_{4}$. After filtration, organics were evaporated and the residue was purified by column chromatography $\left(\mathrm{SiO}_{2}, \mathrm{AcOEt} / \mathrm{CHCl}_{3} 1: 5\right)$ and crystallized from acetone.

\section{Table S1. Solvent screening of the pyrimidine-tetrazine reaction}

\begin{tabular}{|c|c|c|c|}
\hline Entry & Dry Solvent & Yield & Additional Info \\
\hline 1. & 1,4-Dioxane & $21 \%(29 \%)^{a}$ & $50^{\circ} \mathrm{C}$ \\
\hline 2. & DCM & - & $\mathrm{SM}^{\mathrm{a}}$ \\
\hline 3. & DMF & - & SM + oxadiazole ${ }^{a, b}$ \\
\hline 4. & DMSO & - & oxadiazole ${ }^{a, b}$ \\
\hline 5. & HMPA & - & oxadiazole $^{a, b}$ \\
\hline 6. & $\mathrm{MeCN}$ & - & oxadiazole $^{\mathrm{a}, \mathrm{b}}$ \\
\hline 7. & $\mathrm{~m}$-Xylene & $50 \%$ & $50^{\circ} \mathrm{C}$ \\
\hline 8. & $\mathrm{PhCl}$ & $33 \%$ & $50^{\circ} \mathrm{C}$ \\
\hline 9. & $\mathrm{PhCN}$ & $<10 \%$ & oxadiazole $^{\mathrm{a}, \mathrm{b}}$ \\
\hline 10. & $\mathrm{PhH}$ & $46 \%$ & $50^{\circ} \mathrm{C}$ \\
\hline 11. & PhMe & $50 \%$ & $50^{\circ} \mathrm{C}$ \\
\hline 12. & PhOMe & $39 \%$ & $50^{\circ} \mathrm{C}$ \\
\hline 13. & Pyridine & no product & oxadiazole + triazine $e^{a, b, c}$ \\
\hline 14. & THF & $<10 \%$ & oxadiazole + triazine $\mathrm{e}^{\mathrm{a}, \mathrm{b}, \mathrm{c}}$ \\
\hline
\end{tabular}

${ }^{a}$ carried out at RT. ${ }^{b}$ oxadiazole $=2,5$-diphenyl-1,3,4-oxadiazole. ${ }^{c}$ triazine $=2,4,6$-triphenyl-1,3,5-triazine. $\mathrm{RT}=$ room temperature. $\mathrm{SM}=$ starting material.

\section{5,8-Diphenylpyrimido[4,5- $d$ ]pyridazine (6a)}

Purification: $\mathrm{AcOEt} / \mathrm{CHCl}_{3}$ 1:5 $\left(\mathrm{R}_{\mathrm{f}}=0.43\right)$;

Yield: $71 \mathrm{mg}(50 \%)$ as bright yellow powder;

MP: $215.0-215.5^{\circ} \mathrm{C}$;

${ }^{1} \mathrm{H} \mathrm{NMR}\left(400 \mathrm{MHz}, \mathrm{CDCl}_{3}\right): \delta_{\mathrm{H}} 9.77(\mathrm{~s}, 2 \mathrm{H}, 2 \times \mathrm{CH}), 8.29-8.34(\mathrm{~m}, 2 \mathrm{H}, \mathrm{Ph}), 7.86-7.91(\mathrm{~m}$,<smiles>c1ccc(-c2nnc(-c3ccccc3)c3[nH]nnc23)cc1</smiles>
$2 \mathrm{H}, \mathrm{Ph}), 7.58-7.69(\mathrm{~m}, 6 \mathrm{H}, \mathrm{Ph})$;

${ }^{13} \mathrm{C} \mathrm{NMR}\left(100 \mathrm{MHz}, \mathrm{CDCl}_{3}\right): \delta_{\mathrm{C}} 160.6(\mathrm{CH}), 160.3(\mathrm{CH}), 158.5$ (C), 157.8 (C), $143.8(\mathrm{C}), 134.1$ (C), 133.9 (C), $131.4(2 \times \mathrm{CH}), 130.8(\mathrm{CH}), 130.6(3 \times \mathrm{CH}), 129.4(2 \times \mathrm{CH}), 128.6(2 \times \mathrm{CH}), 117.5(\mathrm{C})$;

HRMS (ESI) $\mathrm{m} / \mathrm{z}$ calculated for $\mathrm{C}_{18} \mathrm{H}_{13} \mathrm{~N}_{4}(\mathrm{M}+\mathrm{H})^{+}: 285.1135$, found: 285.1135 . 


\section{2-(Methylthio)-5,8-diphenylpyrimido[4,5- $d$ ]pyridazine (6b)}

Purification: $\mathrm{AcOEt} / \mathrm{CHCl}_{3} 1: 9\left(\mathrm{R}_{\mathrm{f}}=0.40\right)$;

Yield: $71 \mathrm{mg}(43 \%)$ as yellow solid;

MP: $186.5-187.0^{\circ} \mathrm{C}$;

${ }^{1} \mathrm{H}$ NMR $\left(400 \mathrm{MHz}, \mathrm{CDCl}_{3}\right): \delta_{\mathrm{H}} 9.41(\mathrm{~s}, 1 \mathrm{H}, \mathrm{CH}), 8.33-8.39(\mathrm{~m}, 2 \mathrm{H}, \mathrm{Ph}), 7.81-7.87(\mathrm{~m}$,

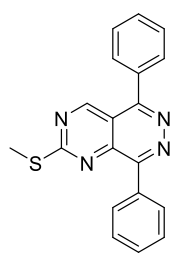

$2 \mathrm{H}, \mathrm{Ph}), 7.54-7.66(\mathrm{~m}, 6 \mathrm{H}, \mathrm{Ph}), 2.66\left(\mathrm{~s}, 3 \mathrm{H}, \mathrm{CH}_{3}\right)$;

${ }^{13} \mathrm{C}$ NMR (100 MHz, CDCl $)$ ): $\delta_{\mathrm{C}} 176.5$ (C), $160.0(\mathrm{CH}), 157.7$ (C), 157.1 (C), 144.2 (C), 134.3 (C), 134.1 (C), $131.2(2 \times \mathrm{CH}), 130.6(\mathrm{CH}), 130.5(2 \times \mathrm{CH}), 130.3(\mathrm{CH}), 129.3(2 \times \mathrm{CH}), 128.3(2 \times \mathrm{CH}), 114.6(\mathrm{C}), 14.9$ $\left(\mathrm{CH}_{3}\right)$;

HRMS (ESI) $\mathrm{m} / \mathrm{z}$ calculated for $\mathrm{C}_{19} \mathrm{H}_{15} \mathrm{~N}_{4} \mathrm{~S}(\mathrm{M}+\mathrm{H})^{+}: 331.1012$, found: 331.1013.

\section{2-Chloro-4-methoxy-5,8-diphenylpyrimido[4,5- $d]$ pyridazine (6c)}

Purification: $\mathrm{AcOEt} / \mathrm{CHCl}_{3}$ 1:8 $\left(\mathrm{R}_{\mathrm{f}}=0.44\right)$;

Yield: $80 \mathrm{mg}$ (46\%) as bright yellow solid;

MP: $174.0-174.5^{\circ} \mathrm{C}$;

${ }^{1} \mathrm{H}$ NMR $\left(400 \mathrm{MHz}, \mathrm{CDCl}_{3}\right): \delta_{\mathrm{H}} 8.25-8.29(\mathrm{~m}, 2 \mathrm{H}, \mathrm{Ph}), 7.59-7.63(\mathrm{~m}, 2 \mathrm{H}, \mathrm{Ph}), 7.55-7.58$

(m, 3H, Ph), $7.49-7.53(\mathrm{~m}, 3 \mathrm{H}, \mathrm{Ph}), 3.95\left(\mathrm{~s}, 3 \mathrm{H}, \mathrm{CH}_{3}\right)$;

${ }^{13} \mathrm{C} \mathrm{NMR}\left(100 \mathrm{MHz}, \mathrm{CDCl}_{3}\right): \delta_{\mathrm{C}} 168.3$ (C), 161.8 (C), 156.8 (C), 156.7 (C), 147.1 (C), 137.7 (C), 134.1 (C), $131.3(2 \times \mathrm{CH}), 130.4(\mathrm{CH}), 129.6(2 \times \mathrm{CH}), 129.5(\mathrm{CH}), 128.4(2 \times \mathrm{CH}), 128.0(2 \times \mathrm{CH}), 108.9(\mathrm{C}), 56.0\left(\mathrm{CH}_{3}\right)$;

HRMS (ESI) $\mathrm{m} / \mathrm{z}$ calculated for $\mathrm{C}_{19} \mathrm{H}_{14} \mathrm{CIN}_{4} \mathrm{~S}(\mathrm{M}+\mathrm{H})^{+}$: 349.0851 , found: 349.0852 .

\section{2-Chloro- $N, N$-dimethyl-5,8-diphenylpyrimido[4,5-d]pyridazin-4-amine (6d)}

Purification: $\mathrm{AcOEt} / \mathrm{CHCl}_{3}$ 1:4 $\left(\mathrm{R}_{\mathrm{f}}=0.40\right)$;

Yield: $60 \mathrm{mg}(33 \%)$ as yellow solid;

MP: $258.5-259.0^{\circ} \mathrm{C}$;

${ }^{1} \mathrm{H}$ NMR $\left(400 \mathrm{MHz}, \mathrm{CDCl}_{3}\right): \delta_{\mathrm{H}} 8.22-8.28(\mathrm{~m}, 2 \mathrm{H}, \mathrm{Ph}), 7.60-7.95$ (bs, $\left.2 \mathrm{H}, \mathrm{Ph}\right), 7.48-7.58$ $(\mathrm{m}, 6 \mathrm{H}, \mathrm{Ph}), 2.25-3.45$ (bs, $\left.6 \mathrm{H}, 2 \times \mathrm{CH}_{3}\right)$;

${ }^{13} \mathrm{C} \mathrm{NMR}\left(100 \mathrm{MHz}, \mathrm{CDCl}_{3}\right): \delta_{\mathrm{C}} 162.6$ (C), 160.3 (C), 155.4 (C), 155.1 (C), 149.1 (C), 137.7 (C), 134.6 (C), $131.0(2 \times \mathrm{CH}), 130.6(\mathrm{CH}), 130.1(\mathrm{CH}), 129.3(2 \times \mathrm{CH}), 129.0(2 \times \mathrm{CH}), 128.4(2 \times \mathrm{CH}), 105.1(\mathrm{C})$, dimethylamino group is not visible at RT but is resolved at $238 \mathrm{~K}$ (42.2 and $38.9 \mathrm{ppm}$, see spectra);

HRMS (ESI) $\mathrm{m} / \mathrm{z}$ calculated for $\mathrm{C}_{20} \mathrm{H}_{17} \mathrm{CIN}_{5}(\mathrm{M}+\mathrm{H})^{+}: 362.1167$, found: 362.1165 .

\section{2-Chloro-4-(ethylthio)-5,8-diphenylpyrimido[4,5-d]pyridazine (6e)}

Purification: $\mathrm{AcOEt} / \mathrm{CHCl}_{3} 1: 8\left(\mathrm{R}_{\mathrm{f}}=0.47\right)$;

Yield: $138 \mathrm{mg}(73 \%)$ as yellow solid;

MP: $154.0-155.0^{\circ} \mathrm{C}$;

${ }^{1} \mathrm{H}$ NMR $\left(400 \mathrm{MHz}, \mathrm{CDCl}_{3}\right): \delta_{\mathrm{H}} 8.21-8.26(\mathrm{~m}, 2 \mathrm{H}, \mathrm{Ph}), 7.53-7.63(\mathrm{~m}, 8 \mathrm{H}, \mathrm{Ph}), 3.13(\mathrm{q}$,

$\left.{ }^{3} J_{H, H}=7.4,2 \mathrm{H}, \mathrm{CH}_{2}\right), 1.26\left(\mathrm{t},{ }^{3} \mathrm{H}_{\mathrm{H}, \mathrm{H}}=7.4,3 \mathrm{H}, \mathrm{CH}_{3}\right)$;

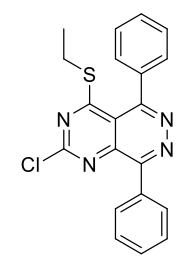


${ }^{13} \mathrm{C} \mathrm{NMR}\left(100 \mathrm{MHz}, \mathrm{CDCl}_{3}\right): \delta_{\mathrm{C}} 178.0$ (C), 160.7 (C), 158.1 (C), 156.9 (C), 144.8 (C), 136.5 (C), 134.2 (C), $131.4(2 \times \mathrm{CH}), 130.5(\mathrm{CH}), 130.4(\mathrm{CH}), 130.2(2 \times \mathrm{CH}), 128.9(2 \times \mathrm{CH}), 128.5(2 \times \mathrm{CH}), 116.4(\mathrm{C}), 27.2\left(\mathrm{CH}_{2}\right)$, $13.1\left(\mathrm{CH}_{3}\right)$;

HRMS (ESI) m/z calculated for $\mathrm{C}_{20} \mathrm{H}_{16} \mathrm{CIN}_{4} \mathrm{~S}(\mathrm{M}+\mathrm{H})^{+}: 379.0779$, found: 379.0777 .

\section{2-Chloro-4-(ethylthio)-5,8-diisopropylpyrimido[4,5- $d$ ]pyridazine (6f)}

Purification: $D C M\left(R_{f}=0.32\right)$;

Yield: $95 \mathrm{mg}(61 \%)$ as light orange solid;

MP: $80.5-81.0^{\circ} \mathrm{C}$;

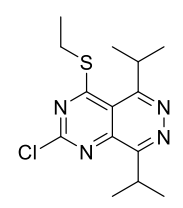

${ }^{1} \mathrm{H} \mathrm{NMR}\left(400 \mathrm{MHz}, \mathrm{CDCl}_{3}\right): \delta_{\mathrm{H}} 4.29\left(\mathrm{sept},{ }^{3} \mathrm{~J}_{\mathrm{H}, \mathrm{H}}=6.6,1 \mathrm{H}, \mathrm{CH}\right), 4.17$ (sept, $\left.{ }^{3} \mathrm{~J}_{\mathrm{H}, \mathrm{H}}=6.9,1 \mathrm{H}, \mathrm{CH}\right), 3.40$ (q, $\left.{ }^{3} J_{\mathrm{H}, \mathrm{H}}=7.4,2 \mathrm{H}, \mathrm{CH}_{2}\right), 1.51\left(\mathrm{~d},{ }^{3} J_{\mathrm{H}, \mathrm{H}}=6.6,6 \mathrm{H}, 2 \times \mathrm{CH}_{3}\right), 1.48\left(\mathrm{t},{ }^{3} J_{\mathrm{H}, \mathrm{H}}=7.4,3 \mathrm{H}, \mathrm{CH}_{3}\right), 1.45\left(\mathrm{~d},{ }^{3} J_{\mathrm{H}, \mathrm{H}}=6.9,6 \mathrm{H}\right.$, $\left.2 \times \mathrm{CH}_{3}\right)$;

${ }^{13} \mathrm{C} \mathrm{NMR}\left(100 \mathrm{MHz}, \mathrm{CDCl}_{3}\right): \delta_{\mathrm{C}} 176.3$ (C), 165.1 (C), 161.8 (C), $159.8(\mathrm{C}), 145.1$ (C), $116.0(\mathrm{C}), 33.8(\mathrm{CH})$, $29.3(\mathrm{CH}), 27.3\left(\mathrm{CH}_{2}\right), 22.8\left(2 \times \mathrm{CH}_{3}\right), 21.8\left(2 \times \mathrm{CH}_{3}\right), 13.2\left(\mathrm{CH}_{3}\right)$;

HRMS (ESI) $\mathrm{m} / \mathrm{z}$ calculated for $\mathrm{C}_{14} \mathrm{H}_{20} \mathrm{ClN}_{4} \mathrm{~S}(\mathrm{M}+\mathrm{H})^{+}: 311.1092$, found: 311.1092 .

\section{2-Chloro-4-(ethylthio)-5-isopropyl-8-phenylpyrimido[4,5-d]pyridazine (6g)}

Purification: $\mathrm{CHCl}_{3} \rightarrow \mathrm{AcOEt} / \mathrm{CHCl}_{3}$ 1:30 $\left(\mathrm{R}_{\mathrm{f}}=0.35\right)$;

Yield: $103 \mathrm{mg}(55 \%)$ as yellow solid; one regioisomer only;

MP: $124.5-125.5^{\circ} \mathrm{C}$;

${ }^{1} \mathrm{H}$ NMR $\left(400 \mathrm{MHz}, \mathrm{CDCl}_{3}\right): \delta_{\mathrm{H}} 8.14-8.18(\mathrm{~m}, 2 \mathrm{H}, 2 \times \mathrm{CH}), 7.52-7.55(\mathrm{~m}, 3 \mathrm{H}, 3 \times \mathrm{CH}), 4.39$

(sept, $\left.{ }^{3} J_{\mathrm{H}, \mathrm{H}}=6.6,1 \mathrm{H}, \mathrm{CH}\right), 3.43\left(\mathrm{q},{ }^{3} J_{\mathrm{H}, \mathrm{H}}=7.4,2 \mathrm{H}, \mathrm{CH}_{2}\right), 1.57\left(\mathrm{~d},{ }^{3} \mathrm{~J}_{\mathrm{H}, \mathrm{H}}=6.6,6 \mathrm{H}, 2 \times \mathrm{CH}_{3}\right), 1.50\left(\mathrm{t},{ }^{3} J_{\mathrm{H}, \mathrm{H}}=\right.$ 7.4, $\left.3 \mathrm{H}, \mathrm{CH}_{3}\right)$;

${ }^{13} \mathrm{C} \mathrm{NMR}\left(100 \mathrm{MHz}, \mathrm{CDCl}_{3}\right): \delta_{\mathrm{C}} 176.4$ (C), 162.4 (C), 159.9 (C), 157.7 (C), 145.3 (C), 134.5 (C), 131.3 $(2 \times \mathrm{CH}), 130.1(\mathrm{CH}), 128.3(2 \times \mathrm{CH}), 116.9(\mathrm{C}), 33.9(\mathrm{CH}), 27.4\left(\mathrm{CH}_{2}\right), 22.9\left(2 \times \mathrm{CH}_{3}\right), 13.1\left(\mathrm{CH}_{3}\right)$;

HRMS (ESI) $\mathrm{m} / \mathrm{z}$ calculated for $\mathrm{C}_{17} \mathrm{H}_{18} \mathrm{ClN}_{4} \mathrm{~S}(\mathrm{M}+\mathrm{H})^{+}: 345.0935$, found: 345.0935 .

\section{2-Chloro-N,N-diethyl-4-(ethylthio)-5-(4-methoxyphenyl)pyrimido[4,5-d]pyridazin-8-amine (6h)}

Purification: $\mathrm{AcOEt} / \mathrm{CHCl}_{3}$ 1:10 $\left(\mathrm{R}_{\mathrm{f}}=0.38\right)$;

Yield: $107 \mathrm{mg}$ (53\%) as bright yellow solid; one regioisomer only;

MP: $137.5-138.0^{\circ} \mathrm{C}$;

${ }^{1} \mathrm{H}$ NMR $\left(400 \mathrm{MHz}, \mathrm{CDCl}_{3}\right): 7.37-7.42(\mathrm{~m}, 2 \mathrm{H}, 2 \times \mathrm{CH}), 6.95-7.01(\mathrm{~m}, 2 \mathrm{H}, 2 \times \mathrm{CH}), 3.94$ (q, $\left.{ }^{3} J_{\mathrm{H}, \mathrm{H}}=6.9,4 \mathrm{H}, \mathrm{NCH}_{2}\right), 3.89\left(\mathrm{~s}, 3 \mathrm{H}, \mathrm{OCH}_{3}\right), 3.07\left(\mathrm{q},{ }^{3} \mathrm{~J}_{\mathrm{H}, \mathrm{H}}=7.4,2 \mathrm{H}, \mathrm{SCH}_{2}\right), 1.38\left(\mathrm{t},{ }^{3} \mathrm{H}_{\mathrm{H}, \mathrm{H}}=6.9\right.$, $\left.6 \mathrm{H}, 2 \times \mathrm{CH}_{3}\right), 1.23\left(\mathrm{t},{ }^{3} \mathrm{~J}_{\mathrm{H}, \mathrm{H}}=7.4,3 \mathrm{H}, \mathrm{CH}_{3}\right)$;

${ }^{13} \mathrm{C}$ NMR (100 MHz, CDCl $)$ ): 176.2 (C), 160.8 (C), 157.3 (C), 154.3 (C), 148.2 (C), 139.3 (C), 131.7 $(2 \times \mathrm{CH}), 129.8(\mathrm{C}), 116.9(\mathrm{C}), 113.9(2 \times \mathrm{CH}), 55.5\left(\mathrm{OCH}_{3}\right), 46.2\left(\mathrm{NCH}_{2}\right), 26.9\left(\mathrm{SCH}_{2}\right), 13.4\left(\mathrm{CH}_{3}\right), 13.3$ $\left(\mathrm{CH}_{3}\right)$;

HRMS (ESI) $\mathrm{m} / \mathrm{z}$ calculated for $\mathrm{C}_{19} \mathrm{H}_{23} \mathrm{ClN}_{5} \mathrm{OS}(\mathrm{M}+\mathrm{H})^{+}: 404.1306$, found: 404.1310 . 


\section{2-Chloro-4-(ethylthio)-5-(4-methoxyphenyl)-8-(piperidin-1-yl)pyrimido[4,5-d]pyridazine (6i)}

Purification: $\mathrm{AcOEt} \mathrm{CHCl}_{3}$ 1:9 $\left(\mathrm{R}_{\mathrm{f}}=0.29\right)$;

Yield: $133 \mathrm{mg}(64 \%)$ as bright yellow solid; one regioisomer only;

MP: $172.5-173.0^{\circ} \mathrm{C}$;

${ }^{1} \mathrm{H}$ NMR $\left(400 \mathrm{MHz}, \mathrm{CDCl}_{3}\right): \delta_{\mathrm{H}} 7.37-7.42(\mathrm{~m}, 2 \mathrm{H}, 2 \times \mathrm{CH}), 6.97-7.02(\mathrm{~m}, 2 \mathrm{H}, 2 \times \mathrm{CH}), 3.96$ $-4.01\left(\mathrm{~m}, 4 \mathrm{H}, \mathrm{NCH}_{2}\right), 3.90\left(\mathrm{~s}, 3 \mathrm{H}, \mathrm{OCH}_{3}\right), 3.08\left(\mathrm{q},{ }^{3} \mathrm{~J}_{\mathrm{H}, \mathrm{H}}=7.4,2 \mathrm{H}, \mathrm{SCH}_{2}\right), 1.72-1.84(\mathrm{~m}, 6 \mathrm{H}$, $\left.3 \times \mathrm{CH}_{2}\right), 1.23\left(\mathrm{t},{ }^{3} \mathrm{~J}_{\mathrm{H}, \mathrm{H}}=7.4,3 \mathrm{H}, \mathrm{CH}_{3}\right)$;

${ }^{13} \mathrm{C}$ NMR (100 MHz, CDCl $)_{3}$ : $\delta_{\mathrm{C}} 177.1$ (C), 161.0 (C), 158.0 (C), 156.5 (C), 150.3 (C), 140.4 (C), 131.7 $(2 \times \mathrm{CH}), 129.5(\mathrm{C}), 117.1(\mathrm{C}), 114.0(2 \times \mathrm{CH}), 55.6\left(\mathrm{OCH}_{3}\right), 50.7\left(2 \times \mathrm{CH}_{2}\right), 27.0\left(\mathrm{CH}_{2}\right), 26.1\left(2 \times \mathrm{CH}_{2}\right), 25.0$ $\left(\mathrm{CH}_{2}\right), 13.3\left(\mathrm{CH}_{3}\right)$;

HRMS (ESI) $\mathrm{m} / \mathrm{z}$ calculated for $\mathrm{C}_{20} \mathrm{H}_{23} \mathrm{ClN}_{5} \mathrm{OS}(\mathrm{M}+\mathrm{H})^{+}:$: 416.1306, found: 416.1306 .

\section{4-(2-Chloro-4-(ethylthio)-5-(4-methoxyphenyl)pyrimido[4,5-d]pyridazin-8-yl)morpholine (6j)}

Purification: $\mathrm{AcOEt} / \mathrm{CHCl}_{3} 1: 3\left(\mathrm{R}_{\mathrm{f}}=0.26\right)$;

Yield: $106 \mathrm{mg}$ (50\%) as yellow solid; one regioisomer only;

MP: $186.0-186.5^{\circ} \mathrm{C}$;

${ }^{1} \mathrm{H}$ NMR $\left(400 \mathrm{MHz}, \mathrm{CDCl}_{3}\right): \delta_{\mathrm{H}} 7.37-7.41(\mathrm{~m}, 2 \mathrm{H}, 2 \times \mathrm{CH}), 6.98-7.02(\mathrm{~m}, 2 \mathrm{H}, 2 \times \mathrm{CH}), 4.04$

$-4.09\left(\mathrm{~m}, 4 \mathrm{H}, \mathrm{OCH}_{2}\right), 3.92-3.97\left(\mathrm{~m}, 4 \mathrm{H}, \mathrm{NCH}_{2}\right), 3.90\left(\mathrm{~s}, 3 \mathrm{H}, \mathrm{OCH}_{3}\right), 3.08\left(\mathrm{q},{ }^{3} \mathrm{~J}_{\mathrm{H}, \mathrm{H}}=7.4\right.$,

$\left.2 \mathrm{H}, \mathrm{SCH}_{2}\right), 1.23\left(\mathrm{t},{ }^{3} \mathrm{~J}_{\mathrm{H}, \mathrm{H}}=7.4,3 \mathrm{H}, \mathrm{CH}_{3}\right)$;

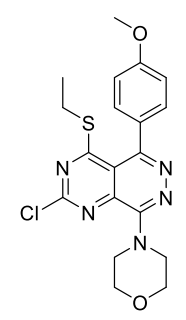

${ }^{13} \mathrm{C} \mathrm{NMR}\left(100 \mathrm{MHz}, \mathrm{CDCl}_{3}\right): \delta_{\mathrm{C}} 177.6$ (C), 161.2 (C), 158.2 (C), 156.0 (C), 151.4 (C), 140.4 (C), 131.6 $(2 \times \mathrm{CH}), 129.2(\mathrm{C}), 117.1(\mathrm{C}), 114.1(2 \times \mathrm{CH}), 67.1\left(\mathrm{OCH}_{2}\right), 55.6\left(\mathrm{OCH}_{3}\right), 49.7\left(\mathrm{NCH}_{2}\right), 27.0\left(\mathrm{SCH}_{2}\right), 13.2$ $\left(\mathrm{CH}_{3}\right)$;

HRMS (ESI) $\mathrm{m} / \mathrm{z}$ calculated for $\mathrm{C}_{19} \mathrm{H}_{21} \mathrm{CIN}_{5} \mathrm{O}_{2} \mathrm{~S}(\mathrm{M}+\mathrm{H})^{+}:$: 418.1099, found: 418.1101.

\section{4-(2-Chloro-4-(ethylthio)-5-phenylpyrimido[4,5-d]pyridazin-8-yl)morpholine (6k)}

Purification: $\mathrm{AcOEt} / \mathrm{CHCl}_{3}$ 1:3 $\left(\mathrm{R}_{\mathrm{f}}=0.43\right)$;

Yield: $95 \mathrm{mg}$ (49\%) as yellow solid; one regioisomer only;

MP: $176.5-177.5^{\circ} \mathrm{C}$;

${ }^{1} \mathrm{H}$ NMR $\left(400 \mathrm{MHz}, \mathrm{CDCl}_{3}\right): \delta_{\mathrm{H}} 7.43-7.54(\mathrm{~m}, 5 \mathrm{H}, \mathrm{Ph}), 4.05-4.10\left(\mathrm{~m}, 4 \mathrm{H}, \mathrm{OCH}_{2}\right), 3.91-$ $3.96\left(\mathrm{~m}, 4 \mathrm{H}, \mathrm{NCH}_{2}\right), 3.06\left(\mathrm{q},{ }^{3} \mathrm{H}_{\mathrm{H}, \mathrm{H}}=7.4,2 \mathrm{H}, \mathrm{SCH}_{2}\right), 1.21\left(\mathrm{t},{ }^{3} \mathrm{~J}_{\mathrm{H}, \mathrm{H}}=7.4,3 \mathrm{H}, \mathrm{CH}_{3}\right)$;

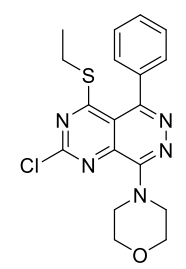

${ }^{13} \mathrm{C} \mathrm{NMR}\left(100 \mathrm{MHz}, \mathrm{CDCl}_{3}\right): \delta_{\mathrm{C}} 177.3$ (C), 158.2 (C), 156.0 (C), 151.5 (C), 140.3 (C), 136.9 (C), 130.2 $(2 \times \mathrm{CH}), 129.8(\mathrm{CH}), 128.6(2 \times \mathrm{CH}), 116.9(\mathrm{C}), 67.0\left(\mathrm{OCH}_{2}\right), 49.7\left(\mathrm{NCH}_{2}\right), 27.0\left(\mathrm{SCH}_{2}\right), 13.1\left(\mathrm{CH}_{3}\right)$;

HRMS (ESI) $\mathrm{m} / \mathrm{z}$ calculated for $\mathrm{C}_{18} \mathrm{H}_{19} \mathrm{CIN}_{5} \mathrm{OS}(\mathrm{M}+\mathrm{H})^{+}: 388.0993$, found: 388.0996 .

\section{4-(2-Chloro-4-(ethylthio)-8-morpholinopyrimido[4,5- $d$ ]pyridazin-5-yl)benzonitrile (6I)}

Purification: $\mathrm{AcOEt} / \mathrm{CHCl}_{3}$ 1:5 $\left(\mathrm{R}_{\mathrm{f}}=0.34\right)$;

Yield: $101 \mathrm{mg}$ (49\%) as pale yellow solid; one regioisomer only;

MP: $222.0-222.5^{\circ} \mathrm{C}$;

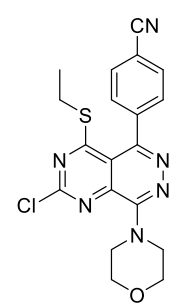


${ }^{1} \mathrm{H}$ NMR $\left(400 \mathrm{MHz}, \mathrm{CDCl}_{3}\right): \delta_{\mathrm{H}} 7.76-7.80(\mathrm{~m}, 2 \mathrm{H}, 2 \times \mathrm{CH}), 7.59-7.63(\mathrm{~m}, 2 \mathrm{H}, 2 \times \mathrm{CH}), 4.13-4.18(\mathrm{~m}$, $\left.4 \mathrm{H}, \mathrm{OCH}_{2}\right), 3.92-3.97\left(\mathrm{~m}, 4 \mathrm{H}, \mathrm{NCH}_{2}\right), 3.13\left(\mathrm{q},{ }^{3} \mathrm{~J}_{\mathrm{H}, \mathrm{H}}=7.4,2 \mathrm{H}, \mathrm{SCH}_{2}\right), 1.25\left(\mathrm{t},{ }^{3} \mathrm{~J}_{\mathrm{H}, \mathrm{H}}=7.4,3 \mathrm{H}, \mathrm{CH}_{3}\right)$;

${ }^{13} \mathrm{C} \mathrm{NMR}\left(100 \mathrm{MHz}, \mathrm{CDCl}_{3}\right): \delta_{\mathrm{C}} 176.3$ (C), 158.4 (C), 156.0 (C), 149.2 (C), 141.7 (C), 140.2 (C), 132.3 $(2 \times \mathrm{CH}), 131.0(2 \times \mathrm{CH}), 118.8(\mathrm{C}), 116.6(\mathrm{C}), 113.6(\mathrm{C}), 67.1\left(\mathrm{OCH}_{2}\right), 49.6\left(\mathrm{NCH}_{2}\right), 27.2\left(\mathrm{SCH}_{2}\right), 13.2\left(\mathrm{CH}_{3}\right)$; HRMS (ESI) $\mathrm{m} / \mathrm{z}$ calculated for $\mathrm{C}_{19} \mathrm{H}_{18} \mathrm{CIN}_{6} \mathrm{OS}(\mathrm{M}+\mathrm{H})^{+}:$: 413.0946, found: 413.0942 .

\section{4-(2-Chloro-4-(ethylthio)-5-(thiophen-2-yl)pyrimido[4,5- $d]$ pyridazin-8-yl)morpholine $(6 \mathrm{~m})$}

Purification: $\mathrm{AcOEt} / \mathrm{CHCl}_{3} 1: 5\left(\mathrm{R}_{\mathrm{f}}=0.40\right)$;

Yield: $98 \mathrm{mg}(50 \%)$ as yellow oil which slowly solidifies upon standing; one regioisomer only;

${ }^{1} \mathrm{H}$ NMR $\left(400 \mathrm{MHz}, \mathrm{CDCl}_{3}\right): \delta_{\mathrm{H}} 7.58\left(\mathrm{dd},{ }^{3} J_{\mathrm{H}, \mathrm{H}}=5.0,{ }^{4} J_{\mathrm{H}, \mathrm{H}}=1.2,1 \mathrm{H}, \mathrm{CH}\right), 7.19\left(\mathrm{dd},{ }^{3} J_{\mathrm{H}, \mathrm{H}}=3.6\right.$, $\left.{ }^{4} J_{\mathrm{H}, \mathrm{H}}=1.2,1 \mathrm{H}, \mathrm{CH}\right), 7.15\left(\mathrm{dd},{ }^{3} \mathrm{~J}_{\mathrm{H}, \mathrm{H}}=5.0,{ }^{3} \mathrm{~J}_{\mathrm{H}, \mathrm{H}}=3.6,1 \mathrm{H}, \mathrm{CH}\right), 4.10-4.15\left(\mathrm{~m}, 4 \mathrm{H}, \mathrm{OCH}_{2}\right)$,

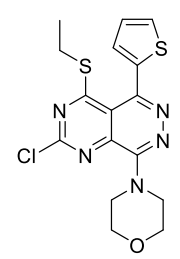
$3.92-3.96\left(\mathrm{~m}, 4 \mathrm{H}, \mathrm{NCH}_{2}\right), 3.12\left(\mathrm{q},{ }^{3} \mathrm{~J}_{\mathrm{H}, \mathrm{H}}=7.4,2 \mathrm{H}, \mathrm{SCH}_{2}\right), 1.27\left(\mathrm{t},{ }^{3} \mathrm{~J}_{\mathrm{H}, \mathrm{H}}=7.4,3 \mathrm{H}, \mathrm{CH}_{3}\right)$;

${ }^{13} \mathrm{C} \mathrm{NMR}\left(100 \mathrm{MHz}, \mathrm{CDCl}_{3}\right): \delta_{\mathrm{C}} 177.3$ (C), 158.1 (C), 156.0 (C), 145.1 (C), 140.0 (C), 136.8 (C), 131.5 $(\mathrm{CH}), 129.1(\mathrm{CH}), 127.5(\mathrm{CH}), 117.8(\mathrm{C}), 67.0\left(\mathrm{OCH}_{2}\right), 49.6\left(\mathrm{NCH}_{2}\right), 27.1\left(\mathrm{SCH}_{2}\right), 13.1\left(\mathrm{CH}_{3}\right)$;

HRMS (ESI) $\mathrm{m} / \mathrm{z}$ calculated for $\mathrm{C}_{16} \mathrm{H}_{17} \mathrm{CIN}_{5} \mathrm{OS}_{2}(\mathrm{M}+\mathrm{H})^{+}$: 394.0558 , found: 394.0559 .

\section{4-(2-Chloro-4-(ethylthio)-5-(furan-2-yl)pyrimido[4,5-d]pyridazin-8-yl)morpholine (6n)}

Purification: $\mathrm{AcOEt} / \mathrm{CHCl}_{3}$ 1:5 $\left(\mathrm{R}_{\mathrm{f}}=0.36\right)$;

Yield: $96 \mathrm{mg}(51 \%)$ as yellow oil which slowly solidifies upon standing; one regioisomer only;

${ }^{1} \mathrm{H}$ NMR $\left(400 \mathrm{MHz}, \mathrm{CDCl}_{3}\right): \delta_{\mathrm{H}} 7.64\left(\mathrm{dd},{ }^{3} \mathrm{~J}_{\mathrm{H}, \mathrm{H}}=1.8 ;{ }^{4} J_{\mathrm{H}, \mathrm{H}}=0.8,1 \mathrm{H}, \mathrm{CH}\right), 6.82\left(\mathrm{dd},{ }^{3} \mathrm{~J}_{\mathrm{H}, \mathrm{H}}=3.4\right.$;

$\left.{ }^{4} J_{\mathrm{H}, \mathrm{H}}=0.8,1 \mathrm{H}, \mathrm{CH}\right), 6.62\left(\mathrm{dd},{ }^{3} \mathrm{~J}_{\mathrm{H}, \mathrm{H}}=3.4 ; 3^{3} \mathrm{H}_{\mathrm{H}}=1.8,1 \mathrm{H}, \mathrm{CH}\right), 4.12-4.16\left(\mathrm{~m}, 4 \mathrm{H}, \mathrm{OCH}_{2}\right)$,

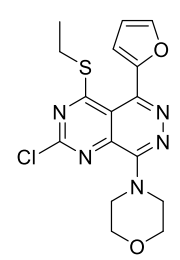

$3.90-3.94\left(\mathrm{~m}, 4 \mathrm{H}, \mathrm{NCH}_{2}\right), 3.17\left(\mathrm{q},{ }^{3} \mathrm{~J}_{\mathrm{H}, \mathrm{H}}=7.4,2 \mathrm{H}, \mathrm{SCH}_{2}\right), 1.30\left(\mathrm{t},{ }^{3} \mathrm{~J}_{\mathrm{H}, \mathrm{H}}=7.4,3 \mathrm{H}, \mathrm{CH}_{3}\right)$;

${ }^{13} \mathrm{C}$ NMR $\left(100 \mathrm{MHz}, \mathrm{CDCl}_{3}\right): \delta_{\mathrm{C}} 177.1$ (C), 158.2 (C), 156.0 (C), 148.5 (C), 143.9 (CH), 141.7 (C), 139.8 (C), $117.9(\mathrm{C}), 112.7(\mathrm{CH}), 112.5(\mathrm{CH}), 67.0\left(\mathrm{OCH}_{2}\right), 49.5\left(\mathrm{NCH}_{2}\right), 26.6\left(\mathrm{SCH}_{2}\right), 13.2\left(\mathrm{CH}_{3}\right)$;

HRMS (ESI) $\mathrm{m} / \mathrm{z}$ calculated for $\mathrm{C}_{16} \mathrm{H}_{17} \mathrm{CIN}_{5} \mathrm{O}_{2} \mathrm{~S}(\mathrm{M}+\mathrm{H})^{+}$: 378.0786 , found: 378.0787 .

General Procedure for the products 6o-u formation: To the solution of pyrimidine $\mathbf{S 8}(0.55 \mathrm{mmol})$ and substituted tetrazine $(0.5 \mathrm{mmol})$ in anhydrous toluene $(5 \mathrm{~mL})$ under argon atmosphere was added dropwise $0.5 \mathrm{M}$ solution of KHMDS in toluene $(0.6 \mathrm{mmol})$ over a period of $10 \mathrm{~min}$. After $12 \mathrm{~h}$ at $\mathrm{RT}$, the reaction mixture was quenched with sat. aq. $\mathrm{NH}_{4} \mathrm{Cl}$, extracted with AcOEt $(3 \times)$ and dried over $\mathrm{Na}_{2} \mathrm{SO}_{4}$. After filtration, organics were evaporated and the residue was purified by column chromatography $\left(\mathrm{SiO}_{2}\right)$ and crystallized from acetone. Here, products 6e-g were isolated after the reaction carried out at $50{ }^{\circ} \mathrm{C}$ producing a bit lower yields instead of RT utilized with pyrimidine $\mathbf{S 4}$.

Representative procedure for preparation of compound $6 \mathrm{p}$ : To the solution of pyrimidine $\mathbf{8 8}$ (115 $\mathrm{mg}, 0.55 \mathrm{mmol}, 1.1 \mathrm{eq}$ ) and substituted tetrazine $\mathbf{S 1 8}(136 \mathrm{mg}, 0.5 \mathrm{mmol}, 1 \mathrm{eq}$.) in anhydrous toluene $(5 \mathrm{~mL})$ under argon atmosphere was added dropwise $0.5 \mathrm{M}$ solution of KHMDS in toluene $(0.6 \mathrm{mmol}$, 1.2 eq.) over a period of $10 \mathrm{~min}$. After $12 \mathrm{~h}$ at RT, the reaction mixture was quenched with sat. aq. $\mathrm{NH}_{4} \mathrm{Cl}$, extracted with AcOEt $(3 \times)$ and dried over $\mathrm{Na}_{2} \mathrm{SO}_{4}$. After filtration, organics were evaporated and the residue was purified by column chromatography $\left(\mathrm{SiO}_{2}\right)$ and crystallized from acetone. 
2-Chloro-N,N-diethyl-4-(ethylthio)-8-(4-methoxyphenyl)pyrimido[4,5-d]pyridazin-5-amine (6o)

Degradation, traces observed only.

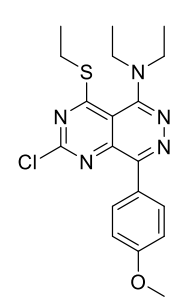

2-Chloro-4-(ethylthio)-8-(4-methoxyphenyl)-5-(piperidin-1-yl)pyrimido[4,5- $d$ ]pyridazine (6p)

Purification: $\mathrm{AcOEt} / \mathrm{CHCl}_{3} 1: 9\left(\mathrm{R}_{\mathrm{f}}=0.51\right)$;

Yield: $117 \mathrm{mg}(56 \%)$ as bright yellow solid; one regioisomer only;

MP: $186.0-187.0^{\circ} \mathrm{C}$;

${ }^{1} \mathrm{H}$ NMR $\left(400 \mathrm{MHz}, \mathrm{CDCl}_{3}\right): \delta_{\mathrm{H}} 8.17-8.22(\mathrm{~m}, 2 \mathrm{H}, 2 \times \mathrm{CH}), 7.02-7.06(\mathrm{~m}, 2 \mathrm{H}, 2 \times \mathrm{CH}), 3.88$ $\left(\mathrm{s}, 3 \mathrm{H}, \mathrm{OCH}_{3}\right), 3.53-3.61\left(\mathrm{~m}, 2 \mathrm{H}, \mathrm{NCH}_{2}\right), 3.28\left(\mathrm{q},{ }^{3} \mathrm{~J}_{\mathrm{H}, \mathrm{H}}=7.4,2 \mathrm{H}, \mathrm{SCH}_{2}\right), 3.17-3.25(\mathrm{~m}, 2 \mathrm{H}$, $\left.\mathrm{NCH}_{2}\right), 1.79-1.98\left(\mathrm{~m}, 5 \mathrm{H}\right.$, piperidine), $1.49-1.61\left(\mathrm{~m}, 1 \mathrm{H}\right.$, piperidine), $1.42\left(\mathrm{t},{ }^{3} \mathrm{~J}_{\mathrm{H}, \mathrm{H}}=7.4\right.$,

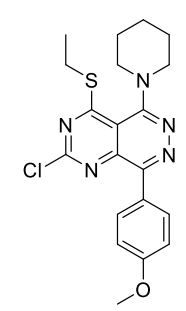
$\left.3 \mathrm{H}, \mathrm{CH}_{3}\right)$;

${ }^{13} \mathrm{C} \mathrm{NMR}\left(100 \mathrm{MHz}, \mathrm{CDCl}_{3}\right): \delta_{\mathrm{C}} 176.3$ (C), 161.0 (C), 159.7 (C), 158.0 (C), 154.0 (C), 146.8 (C), 132.1 $(2 \times \mathrm{CH}), 127.0(\mathrm{C}), 113.8(2 \times \mathrm{CH}), 112.5(\mathrm{C}), 55.5\left(\mathrm{OCH}_{3}\right), 53.4\left(2 \times \mathrm{CH}_{2}\right), 26.0\left(\mathrm{CH}_{2}\right), 25.5\left(2 \times \mathrm{CH}_{2}\right), 24.3$ $\left(\mathrm{CH}_{2}\right), 13.3\left(\mathrm{CH}_{3}\right)$;

HRMS (ESI) m/z calculated for $\mathrm{C}_{20} \mathrm{H}_{23} \mathrm{CIN}_{5} \mathrm{OS}(\mathrm{M}+\mathrm{H})^{+}:$416.1306, found: 416.1307 .

\section{4-(2-Chloro-4-(ethylthio)-8-(4-methoxyphenyl)pyrimido[4,5-d]pyridazin-5-yl)morpholine (6q)}

Purification: $\mathrm{AcOEt} / \mathrm{CHCl}_{3}$ 1:5 $\left(\mathrm{R}_{\mathrm{f}}=0.37\right)$;

Yield: $121 \mathrm{mg}(58 \%)$ as yellow solid; one regioisomer only;

MP: $190.0-191.0^{\circ} \mathrm{C}$;

${ }^{1} \mathrm{H}$ NMR $\left(400 \mathrm{MHz}, \mathrm{CDCl}_{3}\right): \delta_{\mathrm{H}} 8.16-8.22(\mathrm{~m}, 2 \mathrm{H}, 2 \times \mathrm{CH}), 7.02-7.07(\mathrm{~m}, 2 \mathrm{H}, 2 \times \mathrm{CH}), 3.98$

- $4.04\left(\mathrm{~m}, 4 \mathrm{H}, \mathrm{OCH}_{2}\right), 3.89\left(\mathrm{~s}, 3 \mathrm{H}, \mathrm{OCH}_{3}\right), 3.36-3.57\left(\mathrm{~m}, 4 \mathrm{H}, \mathrm{NCH}_{2}\right), 3.30\left(\mathrm{q},{ }^{3} \mathrm{~J}_{\mathrm{H}, \mathrm{H}}=7.4\right.$, $\left.2 \mathrm{H}, \mathrm{SCH}_{2}\right), 1.44\left(\mathrm{t},{ }^{3} \mathrm{~J}_{\mathrm{H}, \mathrm{H}}=7.4,3 \mathrm{H}, \mathrm{CH}_{3}\right)$;

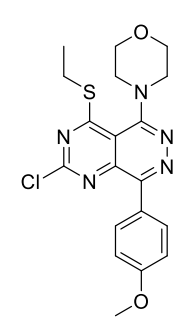

${ }^{13} \mathrm{C} \mathrm{NMR}\left(100 \mathrm{MHz}, \mathrm{CDCl}_{3}\right): \delta_{\mathrm{C}} 175.8$ (C), 161.2 (C), 160.0 (C), 157.2 (C), 155.0 (C), 146.9 (C), 132.2 $(2 \times \mathrm{CH}), 126.7(\mathrm{C}), 113.9(2 \times \mathrm{CH}), 112.5(\mathrm{C}), 66.5\left(\mathrm{OCH}_{2}\right), 55.6\left(\mathrm{OCH}_{3}\right), 52.4\left(\mathrm{NCH}_{2}\right), 26.1\left(\mathrm{SCH}_{2}\right), 13.3$ $\left(\mathrm{CH}_{3}\right)$;

HRMS (ESI) $\mathrm{m} / \mathrm{z}$ calculated for $\mathrm{C}_{19} \mathrm{H}_{21} \mathrm{CIN}_{5} \mathrm{O}_{2} \mathrm{~S}(\mathrm{M}+\mathrm{H})^{+}:$: 418.1099, found: 418.1098.

\section{4-(2-Chloro-4-(ethylthio)-8-phenylpyrimido[4,5-d]pyridazin-5-yl)morpholine (6r)}

Purification: $\mathrm{AcOEt} / \mathrm{CHCl}_{3}$ 1:6 $\left(\mathrm{R}_{\mathrm{f}}=0.42\right)$;

Yield: $82 \mathrm{mg}$ (42\%) as bright yellow solid; one regioisomer only;

MP: $193.5-194.5^{\circ} \mathrm{C}$;

${ }^{1} \mathrm{H}$ NMR $\left(400 \mathrm{MHz}, \mathrm{CDCl}_{3}\right): \delta_{\mathrm{H}} 8.11-8.18(\mathrm{~m}, 2 \mathrm{H}, \mathrm{Ph}), 7.47-7.56(\mathrm{~m}, 3 \mathrm{H}, \mathrm{Ph}), 3.97-4.05$ $\left(\mathrm{m}, 4 \mathrm{H}, \mathrm{OCH}_{2}\right), 3.38-3.61\left(\mathrm{~m}, 4 \mathrm{H}, \mathrm{NCH}_{2}\right), 3.31\left(\mathrm{q},{ }^{3} \mathrm{~J}_{\mathrm{H}, \mathrm{H}}=7.4,2 \mathrm{H}, \mathrm{SCH}_{2}\right), 1.44\left(\mathrm{t},{ }^{3} \mathrm{H}_{\mathrm{H}, \mathrm{H}}=7.4\right.$,

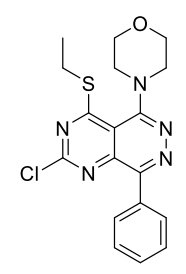
$\left.3 \mathrm{H}, \mathrm{CH}_{3}\right)$; 
${ }^{13} \mathrm{C} \mathrm{NMR}\left(100 \mathrm{MHz}, \mathrm{CDCl}_{3}\right): \delta_{\mathrm{C}} 175.8$ (C), 160.2 (C), 157.5 (C), 155.5 (C), 147.0 (C), 134.2 (C), 130.7 $(2 \times \mathrm{CH}), 129.9(\mathrm{CH}), 128.4(2 \times \mathrm{CH}), 112.4(\mathrm{C}), 66.5\left(\mathrm{OCH}_{2}\right), 52.4\left(\mathrm{NCH}_{2}\right), 26.1\left(\mathrm{SCH}_{2}\right), 13.3\left(\mathrm{CH}_{3}\right)$;

HRMS (ESI) $\mathrm{m} / \mathrm{z}$ calculated for $\mathrm{C}_{18} \mathrm{H}_{19} \mathrm{CIN}_{5} \mathrm{OS}(\mathrm{M}+\mathrm{H})^{+}$: 388.0993, found: 388.0994 .

\section{4-(2-Chloro-4-(ethylthio)-5-morpholinopyrimido[4,5-d]pyridazin-8-yl)benzonitrile (6s)}

Purification: $\mathrm{AcOEt} / \mathrm{CHCl}_{3} 1: 5\left(\mathrm{R}_{\mathrm{f}}=0.32\right)$;

Yield: $81 \mathrm{mg}$ (39\%) as bright yellow solid; one regioisomer only;

MP: $230.0-231.0^{\circ} \mathrm{C}$;

${ }^{1} \mathrm{H}$ NMR $\left(400 \mathrm{MHz}, \mathrm{CDCl}_{3}\right): \delta_{\mathrm{H}} 8.30-8.34(\mathrm{~m}, 2 \mathrm{H}, 2 \times \mathrm{CH}), 7.78-7.83(\mathrm{~m}, 2 \mathrm{H}, 2 \times \mathrm{CH}), 3.97$

$-4.05\left(\mathrm{~m}, 4 \mathrm{H}, \mathrm{OCH}_{2}\right), 3.41-3.67\left(\mathrm{~m}, 4 \mathrm{H}, \mathrm{NCH}_{2}\right), 3.33\left(\mathrm{q},{ }^{3} \mathrm{~J}_{\mathrm{H}, \mathrm{H}}=7.4,2 \mathrm{H}, \mathrm{SCH}_{2}\right), 1.44(\mathrm{t}$,

$\left.{ }^{3} \mathrm{~J}_{\mathrm{H}, \mathrm{H}}=7.4,3 \mathrm{H}, \mathrm{CH}_{3}\right)$;

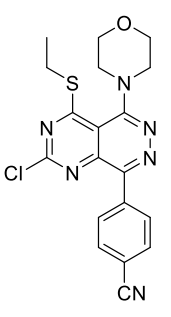

${ }^{13} \mathrm{C} \mathrm{NMR}\left(100 \mathrm{MHz}, \mathrm{CDCl}_{3}\right): \delta_{\mathrm{C}} 176.0$ (C), 160.3 (C), 157.8 (C), 153.2 (C), 147.0 (C), 138.7 (C), 132.1 (2×CH), $131.1(2 \times \mathrm{CH}), 118.9(\mathrm{C}), 113.3(\mathrm{C}), 111.9(\mathrm{C}), 66.4\left(\mathrm{OCH}_{2}\right), 52.3\left(\mathrm{NCH}_{2}\right), 26.2\left(\mathrm{SCH}_{2}\right), 13.3\left(\mathrm{CH}_{3}\right)$; HRMS (ESI) $\mathrm{m} / \mathrm{z}$ calculated for $\mathrm{C}_{19} \mathrm{H}_{18} \mathrm{CIN}_{6} \mathrm{OS}(\mathrm{M}+\mathrm{H})^{+}:$: 413.0946, found: 413.0948 .

\section{4-(2-Chloro-4-(ethylthio)-8-(thiophen-2-yl)pyrimido[4,5-d]pyridazin-5-yl)morpholine (6t)}

\section{Purification: 1. AcOEt/CHCl 3 1:9 $\left(\mathrm{R}_{\mathrm{f}}=0.47\right) ; 2 . \mathrm{AcOEt} / \mathrm{PhCH}_{3}$ 1:9 $\left(\mathrm{R}_{\mathrm{f}}=0.22\right)$;}

Yield: $91 \mathrm{mg}$ (46\%) as orange solid; one regioisomer only;

MP: $160.5-161.5^{\circ} \mathrm{C}$;

${ }^{1} \mathrm{H}$ NMR $\left(400 \mathrm{MHz}, \mathrm{CDCl}_{3}\right): \delta_{\mathrm{H}} 8.60\left(\mathrm{dd},{ }^{3} \mathrm{~J}_{\mathrm{H}, \mathrm{H}}=5.1,{ }^{3} J_{\mathrm{H}, \mathrm{H}}=3.8,1 \mathrm{H}, \mathrm{CH}\right), 7.53\left(\mathrm{dd},{ }^{3} \mathrm{~J}_{\mathrm{H}, \mathrm{H}}=5.1\right.$, $\left.{ }^{4} J_{\mathrm{H}, \mathrm{H}}=1.2,1 \mathrm{H}, \mathrm{CH}\right), 7.20\left(\mathrm{dd},{ }^{3} \mathrm{~J}_{\mathrm{H}, \mathrm{H}}=3.8,{ }^{4} \mathrm{~J}_{\mathrm{H}, \mathrm{H}}=1.2,1 \mathrm{H}, \mathrm{CH}\right), 3.97-4.03\left(\mathrm{~m}, 4 \mathrm{H}, \mathrm{OCH}_{2}\right)$,

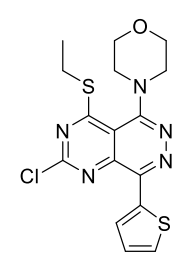
$3.35-3.56\left(\mathrm{~m}, 4 \mathrm{H}, \mathrm{NCH}_{2}\right), 3.31\left(\mathrm{q},{ }^{3} \mathrm{H}_{\mathrm{H}, \mathrm{H}}=7.4,2 \mathrm{H}, \mathrm{SCH}_{2}\right), 1.44\left(\mathrm{t},{ }^{3} \mathrm{~J}_{\mathrm{H}, \mathrm{H}}=7.4,3 \mathrm{H}, \mathrm{CH}_{3}\right)$;

${ }^{13} \mathrm{C}$ NMR $\left(100 \mathrm{MHz}, \mathrm{CDCl}_{3}\right): \delta_{\mathrm{C}} 175.9$ (C), 160.0 (C), 157.1 (C), 150.2 (C), 145.7 (C), 137.5 (C), 131.6 $(\mathrm{CH}), 130.4(\mathrm{CH}), 128.2(\mathrm{CH}), 112.1(\mathrm{C}), 66.4\left(\mathrm{OCH}_{2}\right), 52.4\left(\mathrm{NCH}_{2}\right), 26.2\left(\mathrm{SCH}_{2}\right), 13.3\left(\mathrm{CH}_{3}\right)$;

HRMS (ESI) $\mathrm{m} / \mathrm{z}$ calculated for $\mathrm{C}_{16} \mathrm{H}_{17} \mathrm{CIN}_{5} \mathrm{OS}{ }_{2}(\mathrm{M}+\mathrm{H})^{+}$: 394.0558, found: 394.0558 .

\section{4-(2-Chloro-4-(ethylthio)-8-(furan-2-yl)pyrimido[4,5-d]pyridazin-5-yl)morpholine (6u)}

Purification: $\mathrm{AcOEt} / \mathrm{CHCl}_{3}$ 1:3 $\left(\mathrm{R}_{\mathrm{f}}=0.37\right)$;

Yield: $76 \mathrm{mg}$ ( $40 \%)$ as brownish solid; one regioisomer only;

MP: 197.5 - $198.0^{\circ} \mathrm{C}$;

${ }^{1} \mathrm{H}$ NMR $\left(400 \mathrm{MHz}, \mathrm{CDCl}_{3}\right): \delta_{\mathrm{H}} 7.89-7.92(\mathrm{~m}, 1 \mathrm{H}, \mathrm{CH}), 7.67-7.70(\mathrm{~m}, 1 \mathrm{H}, \mathrm{CH}), 6.62(\mathrm{dd}$, $\left.{ }^{3} J_{H, H}=3.5 ;{ }^{3} J_{H, H}=1.8,1 \mathrm{H}, \mathrm{CH}\right), 3.95-4.02\left(\mathrm{~m}, 4 \mathrm{H}, \mathrm{OCH}_{2}\right), 3.36-3.56\left(\mathrm{~m}, 4 \mathrm{H}, \mathrm{NCH}_{2}\right), 3.30$

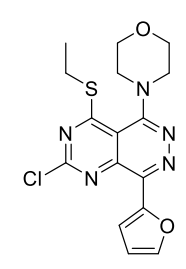
$\left(\mathrm{q},{ }^{3} J_{\mathrm{H}, \mathrm{H}}=7.4,2 \mathrm{H}, \mathrm{SCH}_{2}\right), 1.43\left(\mathrm{t},{ }^{3} \mathrm{~J}_{\mathrm{H}, \mathrm{H}}=7.4,3 \mathrm{H}, \mathrm{CH}_{3}\right)$;

${ }^{13} \mathrm{C}$ NMR $\left(100 \mathrm{MHz}, \mathrm{CDCl}_{3}\right): \delta_{\mathrm{C}} 175.8$ (C), 160.1 (C), 156.8 (C), 147.7 (C), 146.1 (C), 145.6 (C), 145.0 $(\mathrm{CH}), 117.5(\mathrm{CH}), 112.4(\mathrm{CH}), 111.9(\mathrm{C}), 66.4\left(\mathrm{OCH}_{2}\right), 52.3\left(\mathrm{NCH}_{2}\right), 26.1\left(\mathrm{SCH}_{2}\right), 13.3\left(\mathrm{CH}_{3}\right)$;

HRMS (ESI) $\mathrm{m} / \mathrm{z}$ calculated for $\mathrm{C}_{16} \mathrm{H}_{17} \mathrm{CIN}_{5} \mathrm{O}_{2} \mathrm{~S}(\mathrm{M}+\mathrm{H})^{+}$: 378.0786, found: 378.0787 . 


\section{2) Side products and intermediates of fluoropyrimidine $\$ 4$ with tetrazines}

Follow the same protocol as for the products 6 formation. The reaction was carried out at RT with tetrazine S29 leading to $\mathrm{N}$-alkylated product 7, tetrazine S32 produced products $\mathbf{8}$ and $\mathbf{9}$, and tetrazine $\mathbf{S 2 1}$ led to spiro compound $\mathbf{1 0 .}$

\section{1-(2-Chloro-6-(ethylthio)-5-fluoropyrimidin-4-yl)-3,6-dimethoxy-1,2-dihydro-1,2,4,5-tetrazine (7)}

Purification: $\mathrm{AcOEt} / \mathrm{CHCl}_{3} 1: 5\left(\mathrm{R}_{\mathrm{f}}=0.45\right)$;

Yield: $65 \mathrm{mg}(39 \%)$ as yellowish solid;

MP: $125.5-126.5^{\circ} \mathrm{C}$;

${ }^{1} \mathrm{H}$ NMR (400 MHz, $\left.\mathrm{CDCl}_{3}\right): \delta_{\mathrm{H}} 6.33$ (bs, $\left.1 \mathrm{H}, \mathrm{NH}\right), 3.93\left(\mathrm{~s}, 3 \mathrm{H}, \mathrm{OCH}_{3}\right), 3.80\left(\mathrm{~s}, 3 \mathrm{H}, \mathrm{OCH}_{3}\right)$,

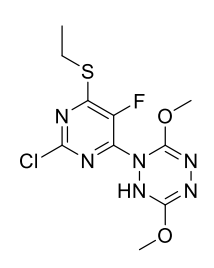
$3.21\left(\mathrm{q},{ }^{3} J_{\mathrm{H}, \mathrm{H}}=7.4,2 \mathrm{H}, \mathrm{CH}_{2}\right), 1.39\left(\mathrm{t},{ }^{3} \mathrm{~J}_{\mathrm{H}, \mathrm{H}}=7.4,3 \mathrm{H}, \mathrm{CH}_{3}\right)$;

${ }^{13} \mathrm{C} \mathrm{NMR}\left(100 \mathrm{MHz}, \mathrm{CDCl}_{3}\right): \delta_{\mathrm{C}} 160.6\left(\mathrm{~s}, \mathrm{C}-\mathrm{OCH}_{3}\right), 159.6\left(\mathrm{~d},{ }^{2} J_{\mathrm{F}, \mathrm{C}}=18.2, \mathrm{C}-\mathrm{S}\right), 153.4\left(\mathrm{~d},{ }^{4} \mathrm{~J}_{\mathrm{F}, \mathrm{C}}=5.0, \mathrm{C}-\mathrm{Cl}\right)$, $149.0\left(\mathrm{~s}, \mathrm{C}-\mathrm{OCH}_{3}\right), 144.8\left(\mathrm{~d},{ }^{2} \mathrm{~J}_{\mathrm{F}, \mathrm{C}}=9.5, \mathrm{C}\right), 144.0\left(\mathrm{~d},{ }^{1} \mathrm{~J}_{\mathrm{F}, \mathrm{C}}=259.7, \mathrm{C}-\mathrm{F}\right), 56.9\left(\mathrm{OCH}_{3}\right), 56.8\left(\mathrm{OCH}_{3}\right), 24.3(\mathrm{~d}$, $\left.{ }^{4} J_{F, C}=1.6, \mathrm{CH}_{2}\right), 14.5\left(\mathrm{CH}_{3}\right)$;

HRMS (ESI) $\mathrm{m} / \mathrm{z}$ calculated for $\mathrm{C}_{10} \mathrm{H}_{13} \mathrm{ClFN}_{6} \mathrm{O}_{2} \mathrm{~S}(\mathrm{M}+\mathrm{H})^{+}: 335.0488$, found: 335.0488 .

\section{3-(2-Chloro-6-(ethylthio)-5-fluoropyrimidin-4-yl)-3,6-bis(4-(trifluoromethyl)phenyl-3,6-dihydro-}

\section{1,2,4,5-tetrazine (8)}

Purification: $\mathrm{CHCl}_{3} /$ pentan 1:1 $\left(\mathrm{R}_{\mathrm{f}}=0.37\right)$;

Yield: $146 \mathrm{mg}(52 \%)$ as yellowish oil;

${ }^{1} \mathrm{H}$ NMR $\left(400 \mathrm{MHz}, \mathrm{CDCl}_{3}\right): \delta_{\mathrm{H}} 8.24-8.29(\mathrm{~m}, 2 \mathrm{H}, \mathrm{Ph}), 7.70-7.77(\mathrm{~m}, 6 \mathrm{H}$, $\mathrm{Ph}), 7.38(\mathrm{~s}, 1 \mathrm{H}, \mathrm{N}-\mathrm{CH}), 3.25\left(\mathrm{q},{ }^{3} \mathrm{~J}_{\mathrm{H}, \mathrm{H}}=7.4,2 \mathrm{H}, \mathrm{CH}_{2}\right), 1.40\left(\mathrm{t},{ }^{3} \mathrm{~J}_{\mathrm{H}, \mathrm{H}}=7.4,3 \mathrm{H}\right.$, $\left.\mathrm{CH}_{3}\right)$;

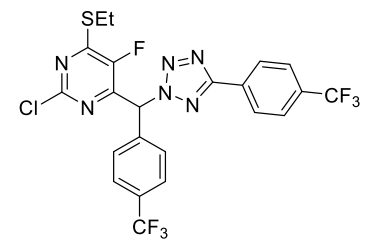

${ }^{13} \mathrm{C}$ NMR $\left(100 \mathrm{MHz}, \mathrm{CDCl}_{3}\right): \delta_{\mathrm{C}} 164.4$ (s, C), $163.9\left(\mathrm{~d},{ }^{2} J_{\mathrm{F}, \mathrm{C}}=17.8, \mathrm{C}-\mathrm{S}\right), 154.9\left(\mathrm{~d},{ }^{4} \mathrm{~J}_{\mathrm{F}, \mathrm{C}}=5.0, \mathrm{C}-\mathrm{Cl}\right), 151.6$ $\left(\mathrm{d},{ }^{1} J_{\mathrm{F}, \mathrm{C}}=265.2, \mathrm{C}-\mathrm{F}\right), 147.8\left(\mathrm{~d},{ }^{2} J_{\mathrm{F}, \mathrm{C}}=12.6, \mathrm{~F}-\mathrm{C}-\mathrm{C}-\mathrm{CH}\right), 136.0(\mathrm{~s}, \mathrm{C}), 132.5\left(\mathrm{q},{ }^{2} \mathrm{~J}_{\mathrm{F}, \mathrm{C}}=32.7, \mathrm{C}\right), 132.3(\mathrm{q}$, $\left.{ }^{2} J_{F, C}=32.9, C\right), 130.5\left(q, J_{F, C}=1.4, C\right), 130.1(s, 2 \times C H), 127.6(s, 2 \times C H), 126.3\left(q, J_{F, C}=3.7,2 \times C H\right)$, $126.1\left(\mathrm{q},{ }^{3} J_{\mathrm{F}, \mathrm{C}}=3.8,2 \times \mathrm{CH}\right), 124.0\left(\mathrm{q},{ }^{1} \mathrm{~J}_{\mathrm{F}, \mathrm{C}}=272.4, \mathrm{C}\right), 123.8\left(\mathrm{q},{ }^{1} \mathrm{~J}_{\mathrm{F}, \mathrm{C}}=272.5, \mathrm{C}\right), 65.2(\mathrm{~s}, \mathrm{~N}-\mathrm{CH}), 24.5(\mathrm{~s}$, $\left.\mathrm{CH}_{2}\right), 14.0\left(\mathrm{~s}, \mathrm{CH}_{3}\right)$;

HRMS (ESI) $\mathrm{m} / \mathrm{z}$ calculated for $\mathrm{C}_{22} \mathrm{H}_{15} \mathrm{ClF}_{7} \mathrm{~N}_{6} \mathrm{~S}(\mathrm{M}+\mathrm{H})^{+}: 563.0650$, found: 563.0649 .

\section{2-Chloro-4-(ethylthio)-5-fluoro-6-(((4-trifluoromethyl)benzylidene)hydrazono)(4-} trifluoromethyl)phenyl)methyl)pyrimidine (9)

Purification: $\mathrm{CHCl}_{3} /$ pentan 1:1 $\left(\mathrm{R}_{\mathrm{f}}=0.40\right)$;

Yield: $13 \mathrm{mg}(5 \%)$ as yellowish oil;

${ }^{1} \mathrm{H}$ NMR $\left(400 \mathrm{MHz}, \mathrm{CDCl}_{3}\right): \delta_{\mathrm{H}} 8.67(\mathrm{~s}, 1 \mathrm{H}, \mathrm{N}=\mathrm{CH}), 7.88-7.92(\mathrm{~m}, 2 \mathrm{H}, \mathrm{Ph}), 7.75-$ $7.79(\mathrm{~m}, 2 \mathrm{H}, \mathrm{Ph}), 7.64-7.72(\mathrm{~m}, 4 \mathrm{H}, \mathrm{Ph}), 3.34\left(\mathrm{q},{ }^{3} \mathrm{~J}_{\mathrm{H}, \mathrm{H}}=7.4,2 \mathrm{H}, \mathrm{CH}_{2}\right), 1.48(\mathrm{t}$, $\left.{ }^{3} \mathrm{~J}_{\mathrm{H}, \mathrm{H}}=7.4,3 \mathrm{H}, \mathrm{CH}_{3}\right)$;

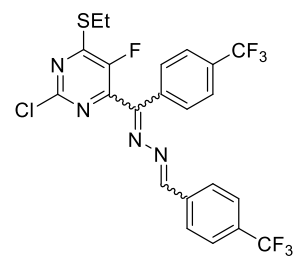

$\left.{ }^{13} \mathrm{C} \mathrm{NMR}(100 \mathrm{MHz}, \mathrm{CDCl})_{3}\right): \delta_{\mathrm{C}} 163.1\left(\mathrm{~d},{ }^{2} \mathrm{~J}_{\mathrm{F}, \mathrm{C}}=18.7, \mathrm{C}-\mathrm{S}\right), 162.0(\mathrm{~s}, \mathrm{~N}=\mathrm{CH}), 158.4\left(\mathrm{~d},{ }^{3} \mathrm{~J}_{\mathrm{F}, \mathrm{C}}=5.0, \mathrm{~F}-\mathrm{C}-\mathrm{C}-\mathrm{C}\right)$, $154.7\left(\mathrm{~d},{ }^{4} J_{\mathrm{F}, \mathrm{C}}=4.2, \mathrm{C}-\mathrm{Cl}\right), 151.4\left(\mathrm{~d},{ }^{1} \mathrm{~J}_{\mathrm{F}, \mathrm{C}}=263.1, \mathrm{C}-\mathrm{F}\right), 147.3\left(\mathrm{~d},{ }^{2} \mathrm{~J}_{\mathrm{F}, \mathrm{C}}=18.8, \mathrm{~F}-\mathrm{C}-\mathrm{C}-\mathrm{C}\right), 137.2\left(\mathrm{q},{ }^{5} \mathrm{~J}_{\mathrm{F}, \mathrm{C}}=\right.$ $1.3, C), 136.9\left(q,{ }^{5} J_{F, C}=1.3, C\right), 133.4\left(q,{ }^{2} J_{F, C}=32.7, C\right), 133.3\left(q,{ }^{2} J_{F, C}=32.7, C\right), 129.4(s, 2 \times C H), 128.9$ 
$(\mathrm{s}, 2 \times \mathrm{CH}), 126.0\left(\mathrm{q},{ }^{3} J_{\mathrm{F}, \mathrm{C}}=3.8,2 \times \mathrm{CH}\right), 125.9\left(\mathrm{q},{ }^{3} J_{\mathrm{F}, \mathrm{C}}=3.8,2 \times \mathrm{CH}\right), 123.9\left(\mathrm{q},{ }^{1} J_{\mathrm{F}, \mathrm{C}}=272.5, \mathrm{C}\right), 123.8(\mathrm{q}$, $\left.{ }^{1} J_{F, C}=272.5, C\right), 24.5\left(\mathrm{~s}, \mathrm{CH}_{2}\right), 14.1\left(\mathrm{~s}, \mathrm{CH}_{3}\right)$;

HRMS (ESI) m/z calculated for $\mathrm{C}_{22} \mathrm{H}_{15} \mathrm{ClF}_{7} \mathrm{~N}_{4} \mathrm{~S}(\mathrm{M}+\mathrm{H})^{+}:$535.0589, found: 535.0590 .

4-(7-Chloro-9-(ethylthio)-10-fluoro-4-(thiophen-2-yl)-2,3,6,8-tetraazaspiro[4.5]deca-1,3,7,9tetraen-1-yl)morpholine (10)
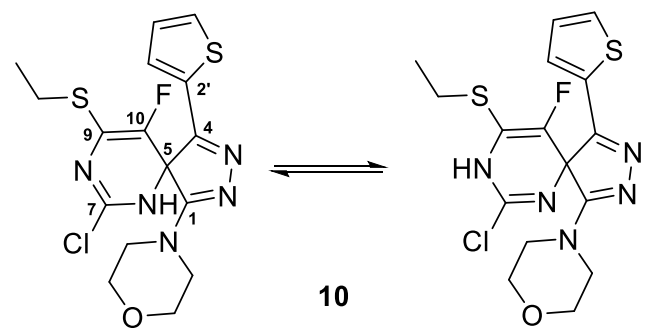<smiles>CCSc1nc(Cl)nc2c(N3CCOCC3)nnc(-c3cccs3)c12</smiles>

Compound $\mathbf{1 0}$ is a mixture of two tautomers - some signals are doubled in NMR spectra and some are broad indicating slow exchange in the NMR time scale to the intermediate tautomer. The ratio of the rotamers is ca 7:3. The major tautomer has the NH signal at $10.3 \mathrm{ppm}$ in ${ }^{1} \mathrm{H}$ NMR spectrum with HMBC crosspeaks to $C 10\left(140.1 \mathrm{ppm}\right.$, doublet, $\left.J_{C-F}=252 \mathrm{~Hz}\right)$ and $C 5\left(73.0 \mathrm{ppm}\right.$, doublet, $\left.J_{\mathrm{C}-\mathrm{C}-\mathrm{F}}=35 \mathrm{~Hz}\right)$. The signal of C9 is also split by the coupling with fluorine $\left(J_{C-C-F}=19 \mathrm{~Hz}\right)$. Small couplings $(2 \mathrm{~Hz})$ of fluorine with $\mathrm{C} 1$ and $\mathrm{C} 4$ are also apparent in ${ }^{13} \mathrm{C}$ NMR spectrum of the major form. The NH proton of the minor tautomer has only one crosspeak in $\mathrm{HMBC}$ spectrum with $\mathrm{C} 10$. Carbon-fluorine couplings in the minor form are similar to those in the major one. The spiro compound in DMSO solution slowly transforms into compound $6 \mathrm{~m}$. The ratio of the two forms of the starting spiro compound is constant during the reaction.

Major tautomer:

${ }^{1} \mathrm{H}$ NMR $\left(500 \mathrm{MHz}, \mathrm{DMSO}-d_{6}\right): \delta_{\mathrm{H}} 10.80$ (bs, $\left.1 \mathrm{H}, \mathrm{H} 6\right), 7.71\left(\mathrm{~m}, 1 \mathrm{H}, \mathrm{H} 5^{\prime}\right), 7.28\left(\mathrm{~m}, 1 \mathrm{H}, \mathrm{H} 3^{\prime}\right), 7.20(\mathrm{~m}, 1 \mathrm{H}$, $\left.\mathrm{H} 4^{\prime}\right), 3.53-3.74\left(\mathrm{~m}, 8 \mathrm{H}, \mathrm{NCH}_{2}\right.$ and $\left.\mathrm{OCH}_{2}\right), 2.86\left(\mathrm{~m}, 2 \mathrm{H}, \mathrm{SCH}_{2}\right), 1.17\left(\mathrm{t},{ }^{3} \mathrm{~J}_{\mathrm{H}, \mathrm{H}}=7.3,3 \mathrm{H}, \mathrm{CH}_{3}\right)$;

${ }^{13} \mathrm{C}$ NMR (126 MHz, DMSO- $\left.d_{6}\right): \delta_{\mathrm{C}} 168.7\left(\mathrm{~d},{ }^{3} \mathrm{~J}_{\mathrm{F}, \mathrm{C}}=1.6, \mathrm{C} 1\right), 158.0\left(\mathrm{~d},{ }^{3} \mathrm{~J}_{\mathrm{F}, \mathrm{C}}=1.7, \mathrm{C} 4\right), 142.4(\mathrm{C} 7), 140.1$ $\left(d,{ }^{1} J_{F, C}=251.9, C 10\right), 132.4\left(C 2^{\prime}\right), 129.4\left(C 5^{\prime}\right), 128.7\left(C 4^{\prime}\right), 126.3\left(C 3^{\prime}\right), 124.6\left(d,{ }^{2} J_{F, C}=19.3, C 9\right), 73.0$ $\left(d,{ }^{2} J_{F, C}=35.0, C 5\right), 65.9\left(\mathrm{OCH}_{2}\right), 46.5\left(\mathrm{NCH}_{2}\right), 23.8\left(d,{ }^{4} J_{\mathrm{F}, \mathrm{C}}=1.7, \mathrm{SCH}_{2}\right), 16.3\left(\mathrm{CH}_{3}\right)$.

Minor tautomer:

${ }^{1} \mathrm{H}$ NMR (500 MHz, DMSO- $\left.d_{6}\right): \delta_{H} 10.29\left(\mathrm{~d},{ }^{4} \mathrm{~J}_{\mathrm{F}, \mathrm{H}}=3.3,1 \mathrm{H}, \mathrm{H6}\right), 7.76\left(\mathrm{~m}, 1 \mathrm{H}, \mathrm{H} 5^{\prime}\right), 7.31\left(\mathrm{~m}, 1 \mathrm{H}, \mathrm{H} 3^{\prime}\right)$, $7.23\left(\mathrm{dd},{ }^{3} \mathrm{~J}_{\mathrm{H}, \mathrm{H}}=5.0\right.$ and $\left.3.8,1 \mathrm{H}, \mathrm{H} 4^{\prime}\right), 3.53-3.74\left(\mathrm{~m}, 8 \mathrm{H}, \mathrm{NCH}_{2}\right.$ and $\left.\mathrm{OCH}_{2}\right), 2.89\left(\mathrm{q},{ }^{3} \mathrm{~J}_{\mathrm{H}, \mathrm{H}}=7.3,2 \mathrm{H}\right.$, $\left.\mathrm{SCH}_{2}\right), 1.17\left(\mathrm{t},{ }^{3} \mathrm{~J}_{\mathrm{H}, \mathrm{H}}=7.3,3 \mathrm{H}, \mathrm{CH}_{3}\right)$;

${ }^{13} \mathrm{C}$ NMR (126 MHz, DMSO- $\left.d_{6}\right): \delta_{\mathrm{C}} 170.4(\mathrm{C} 1), 159.2(\mathrm{C} 4), 142.9(\mathrm{C} 7), 141.3\left(\mathrm{~d},{ }^{1} \mathrm{~J}_{\mathrm{F}, \mathrm{C}}=253.5, \mathrm{C} 10\right), 133.0$ (C2'), $128.9\left(\mathrm{C}^{\prime}\right), 128.4\left(\mathrm{C} 4^{\prime}\right), 126.0\left(\mathrm{C} 3^{\prime}\right), 117.1\left(\mathrm{~d},{ }^{2} \mathrm{~J}_{\mathrm{F}, \mathrm{C}}=30.1, \mathrm{C} 9\right), 76.9\left(\mathrm{~d},{ }^{2} \mathrm{~J}_{\mathrm{F}, \mathrm{C}}=24.2, \mathrm{C} 5\right), 65.9$ $\left(\mathrm{OCH}_{2}\right), 46.3\left(\mathrm{NCH}_{2}\right), 26.8\left(\mathrm{SCH}_{2}\right), 15.3\left(\mathrm{CH}_{3}\right)$.

Tetrazines S11 - S15, S24, S26, S28 and S30 were unsuccessful in the annulation reaction and led to decomposition only. However, tetrazines $\mathbf{S 2 3}, \mathbf{S 2 5}$ and $\mathbf{S 2 7}$ with fluoropyrimidine $\mathbf{S 4}$ afforded in small amounts red solid 11. As the best starting material for such reaction appeared to be tetrazine S27 with pyrrole substitution ( $15 \%$ yield). 


\section{4-(6-(2-Chloro-6-(ethylthio)-5-fluoropyrimidin-4-yl)-1,2,4,5-tetrazin-3-yl)morpholine (11)}

Purification: $\mathrm{AcOEt} / \mathrm{CHCl}_{3}$ 1:9 $\left(\mathrm{R}_{\mathrm{f}}=0.29\right)$;

${ }^{1} \mathrm{H} \mathrm{NMR}\left(400 \mathrm{MHz}, \mathrm{CDCl}_{3}\right): \delta_{\mathrm{H}} 4.10-4.14\left(\mathrm{~m}, 4 \mathrm{H}, \mathrm{OCH}_{2}\right), 3.85-3.89\left(\mathrm{~m}, 4 \mathrm{H}, \mathrm{NCH}_{2}\right)$, $3.30\left(\mathrm{q},{ }^{3} \mathrm{~J}_{\mathrm{H}, \mathrm{H}}=7.4,2 \mathrm{H}, \mathrm{SCH}_{2}\right), 1.44\left(\mathrm{t},{ }^{3} \mathrm{~J}_{\mathrm{H}, \mathrm{H}}=7.4,3 \mathrm{H}, \mathrm{CH}_{3}\right)$;

${ }^{13} \mathrm{C}$ NMR $\left(100 \mathrm{MHz}, \mathrm{CDCl}_{3}\right): \delta_{\mathrm{C}} 164.8\left(\mathrm{~d},{ }^{2} \mathrm{~J}_{\mathrm{F}, \mathrm{C}}=18.6, \mathrm{C}-\mathrm{S}\right), 158.9\left(\mathrm{~s}, \underline{\mathrm{C}}-\mathrm{N}-\mathrm{CH}_{2}\right), 156.5$

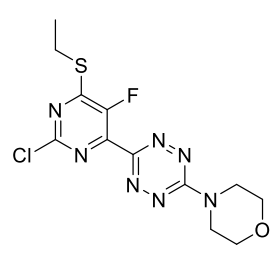
$\left(d,{ }^{3} J_{F, C}=7.7, F-C-C-C\right), 154.8\left(d,{ }^{4} J_{F, C}=4.9, C-C l\right), 152.2\left(d,{ }^{1} J_{F, C}=271.8, C-F\right), 143.5\left(d,{ }^{2} J_{F, C}=9.7, F-C-C-\right.$ C), $66.6\left(\mathrm{~s}, \mathrm{OCH}_{2}\right), 44.0\left(\mathrm{~s}, \mathrm{NCH}_{2}\right), 24.5\left(\mathrm{~d},{ }^{4} \mathrm{~J}_{\mathrm{F}, \mathrm{C}}=1.6, \mathrm{SCH}_{2}\right), 14.1\left(\mathrm{~s}, \mathrm{CH}_{3}\right)$;

HRMS (ESI) $\mathrm{m} / \mathrm{z}$ calculated for $\mathrm{C}_{12} \mathrm{H}_{14} \mathrm{CIFN}_{7} \mathrm{OS}(\mathrm{M}+\mathrm{H})^{+}:$358.0648, found: 358.0649 . 


\section{3) Determination of regioselectivity}

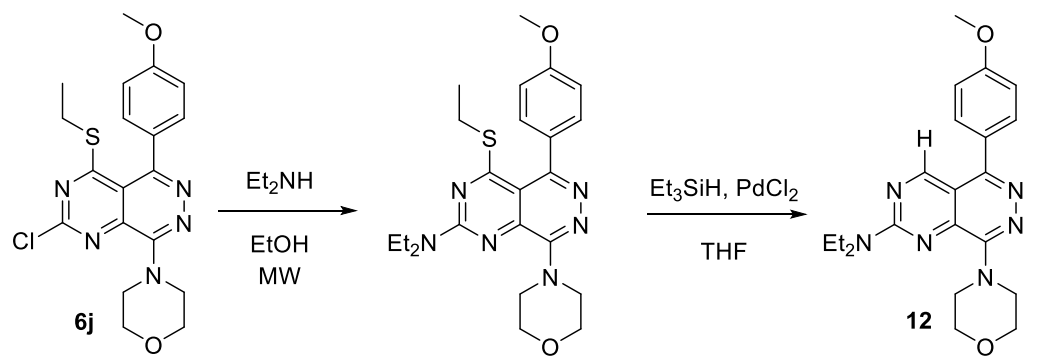

\section{$\mathrm{N}, \mathrm{N}$-Diethyl-5-(4-methoxyphenyl)-8-morpholinopyrimido[4,5- $d$ ]pyridazin-2-amine (12)}

A solution of $6 \mathbf{j}(280 \mathrm{mg}, 0.67 \mathrm{mmol})$ and diethylamine $(0.42 \mathrm{~mL}, 4 \mathrm{mmol})$ in ethanol $(10 \mathrm{~mL})$ was heated in a sealed microwave reactor at $140{ }^{\circ} \mathrm{C}$ for $1 \mathrm{~h}$. The reaction mixture was concentrated in vacuo and purified $\left(\mathrm{SiO}_{2}\right.$, AcOEt/toluene 4:1). The obtained intermediate $(243 \mathrm{mg}, 80 \%)$ confirmed by UPLC measurement was dissolved in dry THF $(7 \mathrm{~mL})$ and palladium(II) chloride $(5 \mathrm{mg})$ was added. To this mixture under argon, triethylsilane $(213 \mu \mathrm{L}, 1.33 \mathrm{mmol})$ was added dropwise at RT and the mixture was stirred for $15 \mathrm{~h}$. Then, water $(2 \mathrm{~mL})$ was carefully added and after gas evolution, the mixture was diluted with water $(30 \mathrm{~mL})$ and extracted with ethylacetate $(3 \times 90 \mathrm{~mL})$. Combined organic phases were dried over $\mathrm{Na}_{2} \mathrm{SO}_{4}$ and evaporated. The residue was purified by column chromatography $\left(\mathrm{SiO}_{2}, \mathrm{AcOEt} /\right.$ toluene $\left.10: 1\right)$ to yield $140 \mathrm{mg}$ (70\%) of 12 as yellow oil which was triturated with diethylether to afford yellow solid.

\section{Region of ROESY spectrum of compound 12 with highlighted indicative cross-peaks.}

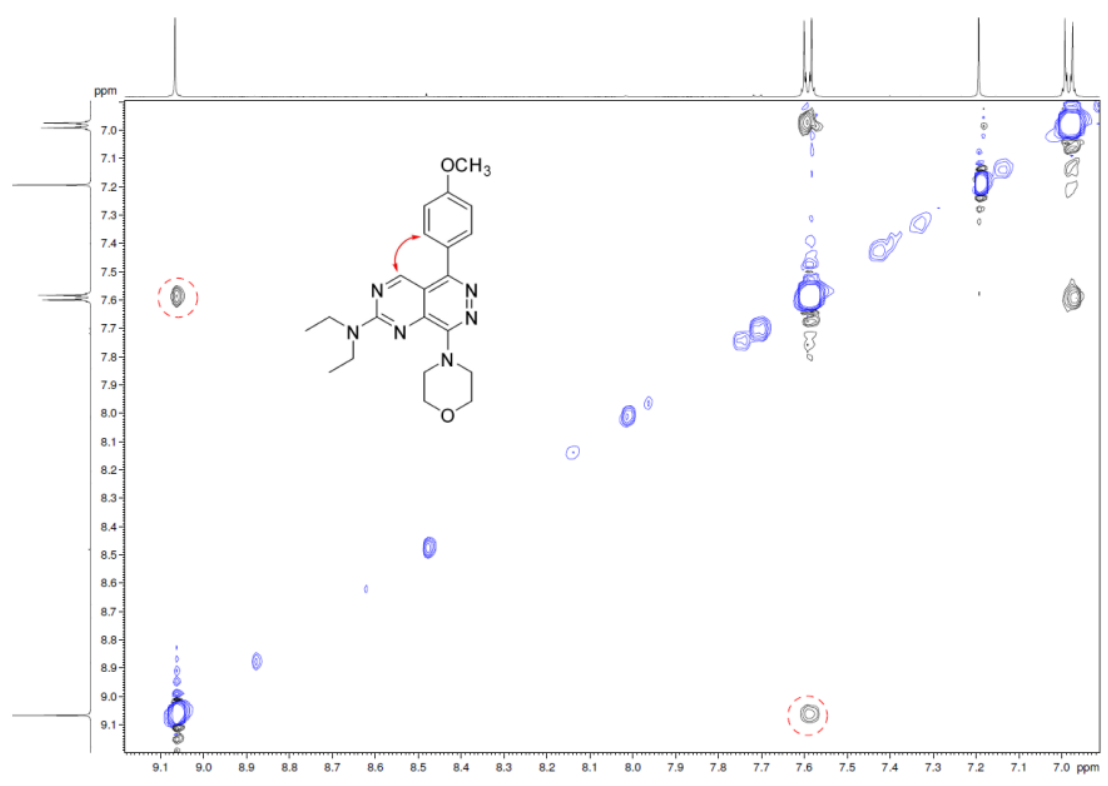

MP: $138.5-139^{\circ} \mathrm{C}$;

${ }^{1} \mathrm{H}$ NMR $\left(400 \mathrm{MHz}, \mathrm{CDCl}_{3}\right): \delta_{\mathrm{H}} 9.13(\mathrm{~s}, 1 \mathrm{H}, \mathrm{H} 4), 7.69-7.63(\mathrm{~m}, 2 \mathrm{H}, 2 \times \mathrm{CH}), 7.09-7.03(\mathrm{~m}, 2 \mathrm{H}, 2 \times \mathrm{CH})$, $4.05-3.99\left(\mathrm{~m}, 4 \mathrm{H}, \mathrm{OCH}_{2}\right), 3.98-3.92\left(\mathrm{~m}, 4 \mathrm{H}, \mathrm{NCH}_{2}\right), 3.89\left(\mathrm{~s}, 3 \mathrm{H}, \mathrm{OCH}_{3}\right), 3.72\left(\mathrm{~m}, 4 \mathrm{H}, 2 \times \mathrm{CH}_{2}\right), 1.30-$ $1.21\left(\mathrm{~m}, 6 \mathrm{H}, 2 \times \mathrm{CH}_{3}\right)$;

${ }^{13} \mathrm{C} \mathrm{NMR}\left(100 \mathrm{MHz}, \mathrm{CDCl}_{3}\right): \delta_{\mathrm{C}} 161.9(\mathrm{CH}), 160.7$ (C), 159.7 (C), 155.9 (C), 152.9 (C), 143.7 (C), 131.3 $(2 \times \mathrm{CH}), 127.8(\mathrm{C}), 114.3(2 \times \mathrm{CH}), 112.0(\mathrm{C}), 67.2\left(\mathrm{OCH}_{2}\right), 55.6\left(\mathrm{OCH}_{3}\right), 49.3\left(\mathrm{NCH}_{2}\right), 43.1$ and 42.8 $\left(\underline{\mathrm{C}} \mathrm{H}_{2} \mathrm{CH}_{3}\right), 13.5$ and $12.6\left(\mathrm{CH}_{2} \underline{\mathrm{C}} \mathrm{H}_{3}\right)$;

HRMS (ESI) $\mathrm{m} / \mathrm{z}$ calculated for $\mathrm{C}_{21} \mathrm{H}_{27} \mathrm{~N}_{6} \mathrm{O}_{2}(\mathrm{M}+\mathrm{H})^{+}: 395.2190$, found: 395.2190 . 


\section{$\mathrm{N}, \mathrm{N}$-Diethyl-8-(4-methoxyphenyl)-5-morpholinopyrimido[4,5-d]pyridazin-2-amine (13)}

A solution of $6 q(100 \mathrm{mg}, 0.24 \mathrm{mmol})$ and diethylamine $(0.12 \mathrm{~mL}, 1.2 \mathrm{mmol})$ in ethanol $(4 \mathrm{~mL})$ was heated in a sealed microwave reactor at $140{ }^{\circ} \mathrm{C}$ for $1 \mathrm{~h}$. The reaction mixture was concentrated in vacuo and purified $\left(\mathrm{SiO}_{2}, \mathrm{AcOEt} /\right.$ toluene $\left.7: 3\right)$. The obtained intermediate (109 mg, quant.) confirmed by UPLC measurement was dissolved in dry THF $(6 \mathrm{~mL})$ and palladium(II) chloride $(2 \mathrm{mg})$ was added. To this mixture under argon, triethylsilane $(100 \mu \mathrm{L}, 0.62 \mathrm{mmol})$ was added dropwise at RT and the mixture was stirred for $15 \mathrm{~h}$. Then, the same amounts of palladium(II) chloride and triethylsilane were added and stirred for additional $15 \mathrm{~h}$. Water $(2 \mathrm{~mL})$ was carefully added and after gas evolution the mixture was diluted with water $(30 \mathrm{~mL})$ and extracted with ethyl acetate $(2 \times 90 \mathrm{~mL})$. Combined organic phases were dried over $\mathrm{Na}_{2} \mathrm{SO}_{4}$ and evaporated. The residue was purified by column chromatography $\left(\mathrm{SiO}_{2}, \mathrm{AcOEt} /\right.$ toluene $\left.2: 1\right)$ to yield $53 \mathrm{mg}(56 \%)$ of $\mathbf{1 3}$ as yellow oil which was triturated with diethylether to afford yellow solid.
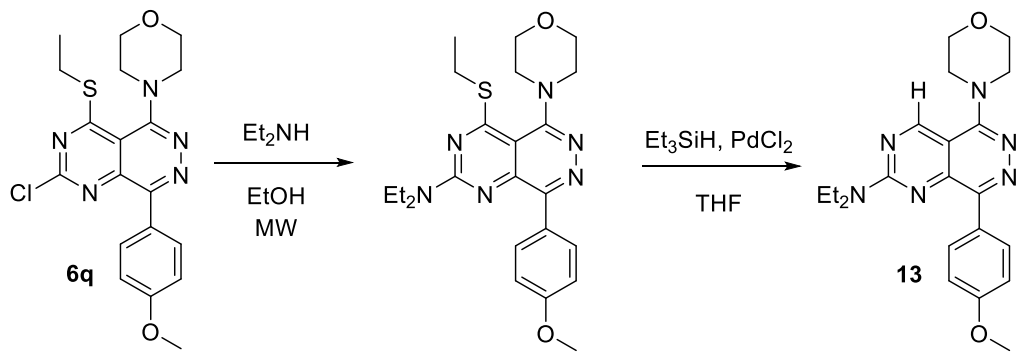

MP: $175-176.5^{\circ} \mathrm{C}$;

${ }^{1} \mathrm{H}$ NMR $\left(400 \mathrm{MHz}, \mathrm{CDCl}_{3}\right): \delta_{\mathrm{H}} 9.17(\mathrm{~s}, 1 \mathrm{H}, \mathrm{H} 4), 8.30-8.33(\mathrm{~m}, 2 \mathrm{H}, 2 \times \mathrm{CH}), 6.98-7.01(\mathrm{~m}, 2 \mathrm{H}, 2 \times \mathrm{CH})$, $3.93-3.97\left(\mathrm{~m}, 4 \mathrm{H}, \mathrm{OCH}_{2}\right), 3.88\left(\mathrm{~s}, 3 \mathrm{H}, \mathrm{OCH}_{3}\right), 3.76\left(\mathrm{q},{ }^{3} \mathrm{~J}_{\mathrm{H}, \mathrm{H}}=7.1,2 \mathrm{H}, \mathrm{CH}_{2} \mathrm{CH}_{3}\right), 3.70\left(\mathrm{q},{ }^{3} \mathrm{~J}_{\mathrm{H}, \mathrm{H}}=7.1,2 \mathrm{H}\right.$, $\left.\mathrm{CH}_{2} \mathrm{CH}_{3}\right), 3.60-3.65\left(\mathrm{~m}, 4 \mathrm{H}, \mathrm{NCH}_{2}\right), 1.22-1.29\left(\mathrm{~m}, 6 \mathrm{H}, 2 \times \mathrm{CH}_{3}\right)$;

${ }^{13} \mathrm{C} \mathrm{NMR}\left(100 \mathrm{MHz}, \mathrm{CDCl}_{3}\right)$ : $\delta_{\mathrm{C}} 160.6$ (C), 160.4 (C), $159.5(\mathrm{CH}), 157.5$ (C), 153.6 (C), 149.0 (C), 131.6 $(2 \times \mathrm{CH}), 127.9(\mathrm{C}), 113.3(2 \times \mathrm{CH}), 106.5(\mathrm{C}), 67.0\left(\mathrm{OCH}_{2}\right), 55.5\left(\mathrm{OCH}_{3}\right), 51.9\left(\mathrm{NCH}_{2}\right), 43.0$ and 42.7 $\left(\mathrm{CH}_{2} \mathrm{CH}_{3}\right), 13.5$ and $12.7\left(\mathrm{CH}_{2} \underline{\mathrm{CH}_{3}}\right)$;

HRMS (ESI) $\mathrm{m} / \mathrm{z}$ calculated for $\mathrm{C}_{21} \mathrm{H}_{27} \mathrm{~N}_{6} \mathrm{O}_{2}(\mathrm{M}+\mathrm{H})^{+}: 395.2190$, found: 395.2190 .

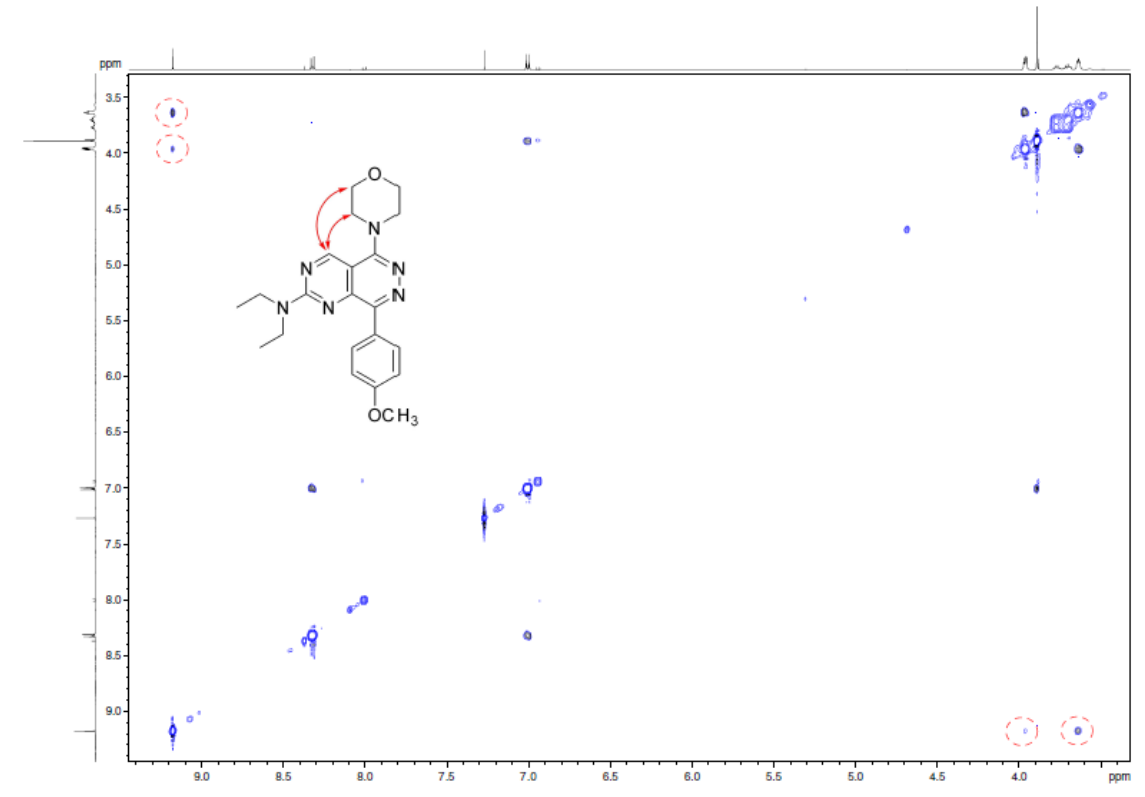




\section{4) Following the reaction by HPLC-MS and NMR measurements}

Reaction of pyrimidine $\mathbf{S 4}$ with diphenyltetrazine 5:

HPLC-MS measurement revealed an intermediate of the reaction with mass corresponding to the sum of the starting pyrimidine and tetrazine. The structure of this intermediate was determined by NMR spectroscopy of the reaction mixture in acetonitrile acquired at $-20{ }^{\circ} \mathrm{C}$ (to slow down the progress of the reaction). The intermediate was identified as 6-(2-chloro-6-(ethylthio)-5fluoropyrimidin-4-yl)-3,6-diphenyl-1,6-dihydro-1,2,4,5-tetrazine (14) with these characteristic NMR signals: $\delta_{\mathrm{C}} 163.0\left(\mathrm{~d},{ }^{2} \mathrm{~J}_{\mathrm{C}, \mathrm{F}}=17.9, \mathrm{C} 6^{\prime}\right), 152.8(\mathrm{C} 3), 152.4\left(\mathrm{~d},{ }^{1} \mathrm{~J}_{\mathrm{C}, \mathrm{F}}=263.2, \mathrm{C} 5^{\prime}\right), 149.6\left(\mathrm{~d},{ }^{2} \mathrm{~J}_{\mathrm{C}, \mathrm{F}}=11.2, \mathrm{C} 4^{\prime}\right)$, $76.8\left(\mathrm{~d},{ }^{3} \mathrm{~J}_{\mathrm{C}, \mathrm{F}}=5.4, \mathrm{C} 6\right) ; \delta_{\mathrm{H}} 8.97(\mathrm{~s}, 1 \mathrm{H}, \mathrm{H} 1)$. The structure was confirmed by an observation of indicative $\mathrm{HMBC}$ crosspeaks, such as $\mathrm{H} 1-\mathrm{C} 6, \mathrm{H} 1-\mathrm{C} 3$ and $\mathrm{SCH}_{2}-\mathrm{C} 6$ '.

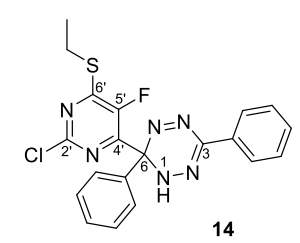

The concentration of compound $\mathbf{1 4}$ gradually decreased and, simultaneously, the concentration of the final product of the reaction (compound 6e) increased. A side-product 15 with structure analogical to that of compound $\mathbf{8}$ was also identified in the reaction mixture.

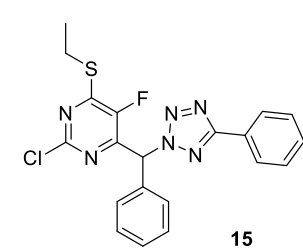

15

Reaction of pyrimidine $\mathbf{S 4}$ with tetrazine $\mathbf{S 1 8}$ :

HPLC-MS measurement revealed a slow formation of the product including a significant presence of the spiro intermediate, which we further verified by NMR spectroscopy. The reaction aliquot was quenched after $20 \mathrm{~min}$ and the sample after quench was followed in time at r.t. 


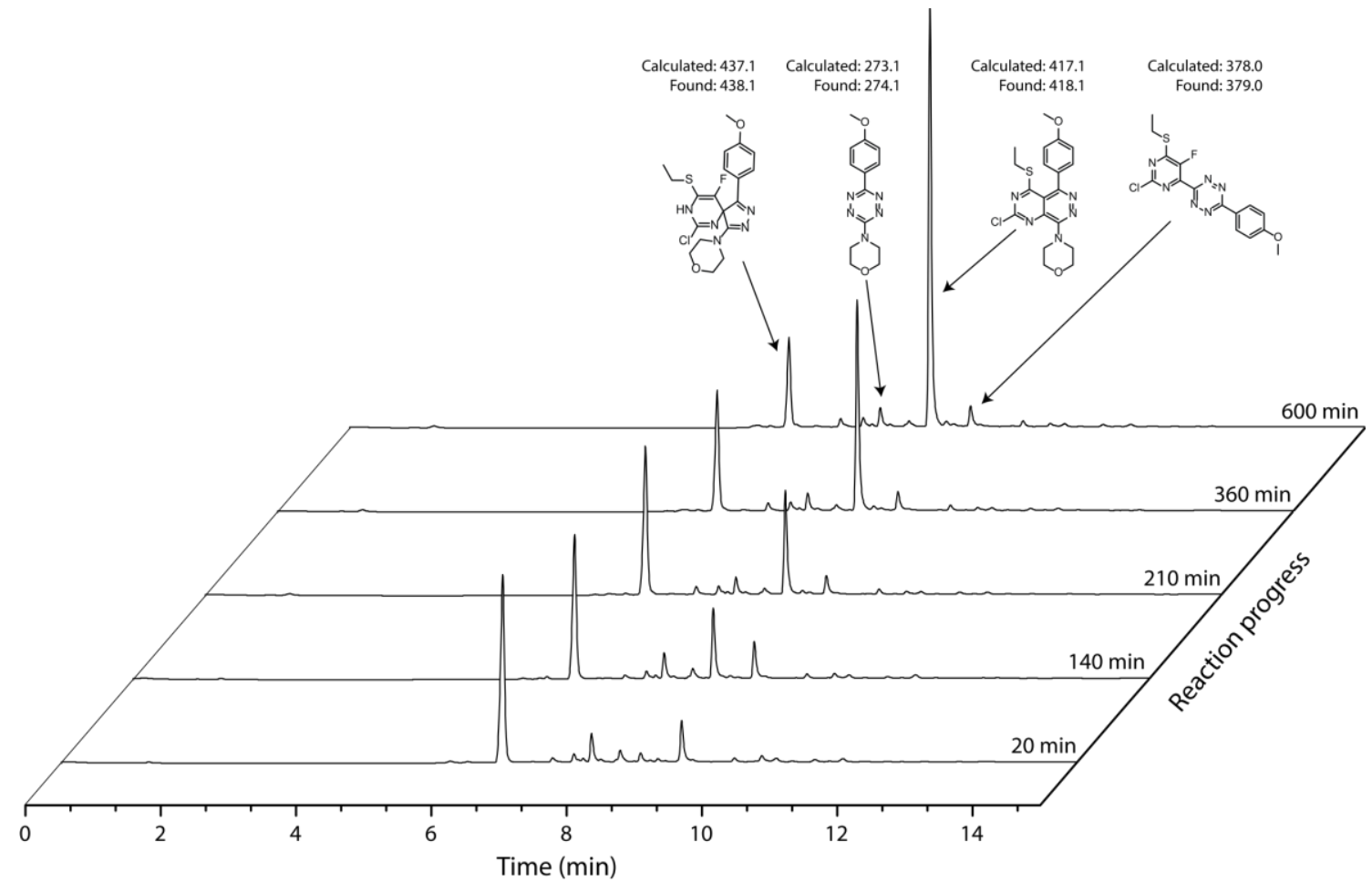

However, when the reaction mixture was quenched after $18 \mathrm{~h}$, it looked almost identical with the previous example (after $20 \mathrm{~min}$ ). Then we heated up the quenched sample and followed in time. The difference is obvious and it implies that the product is rapidly formed only after work-up with water as an accelerator.

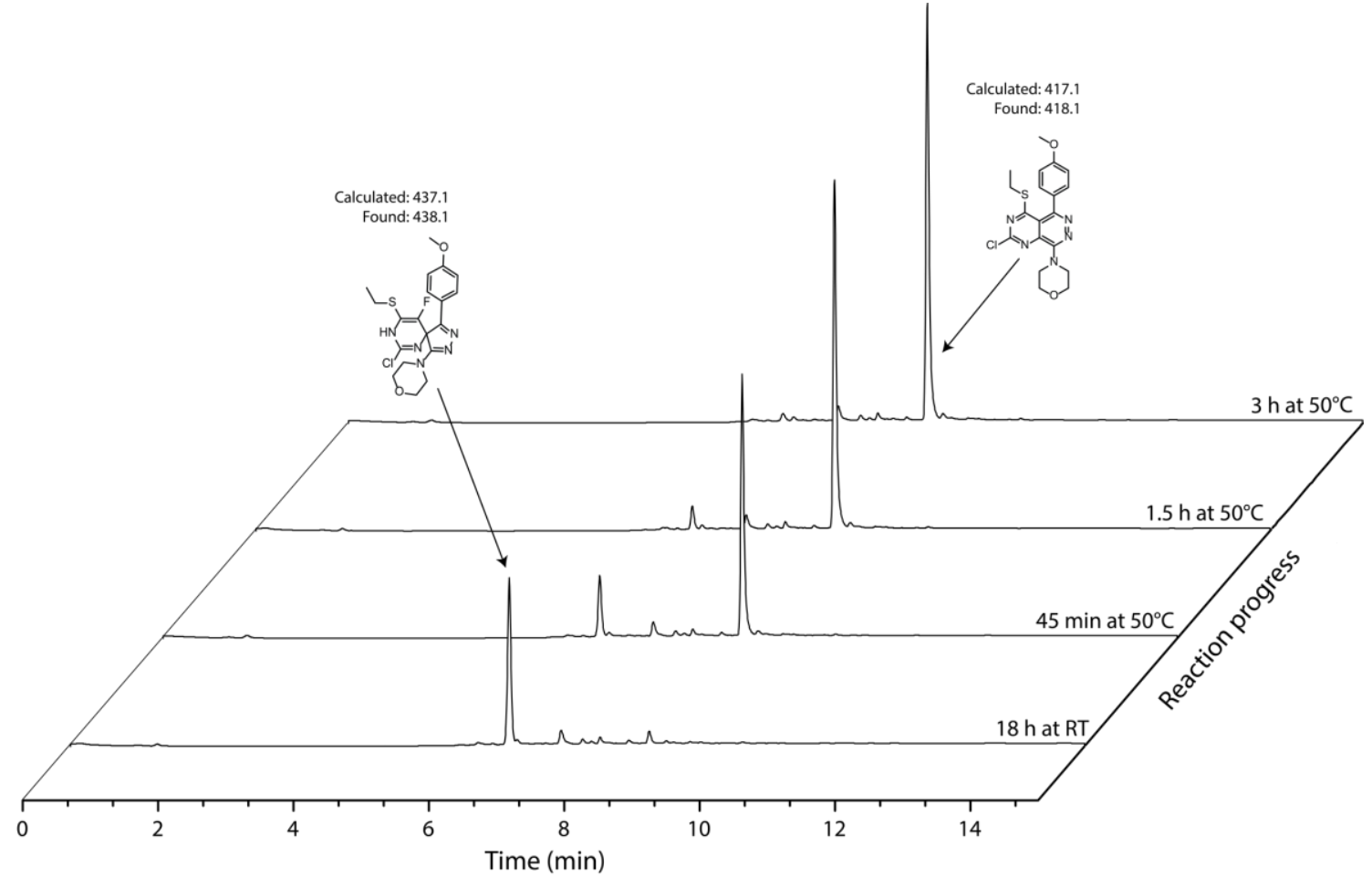


The structure of the spiro intermediate 16 and of the side product $\mathbf{1 7}$ was determined by NMR spectroscopy of the reaction mixture in acetonitrile acquired at $-20^{\circ} \mathrm{C}$ (to slow down the progress of the reaction). The structure of compound 16 is similar to that of 10 and these signals were found to be crucial for the structure determination: $\delta_{\mathrm{C}} 157.6\left(\mathrm{~d},{ }^{3} \mathrm{~J}_{\mathrm{F}, \mathrm{C}}=2.5, \mathrm{C} 4\right), 140.4\left(\mathrm{~d},{ }^{1} J_{\mathrm{F}, \mathrm{C}}=251.6, \mathrm{C} 10\right)$, $124.6\left(d,{ }^{2} J_{F, C}=18.7, C 9\right), 73.1\left(d,{ }^{2} J_{F, C}=34.8, C 5\right)$.<smiles></smiles><smiles>CCSc1nc(Cl)nc(-c2nnc(-c3ccc(OC)cc3)nn2)c1F</smiles> 


\section{5) Computational methods and supplemental data}

All of the structures were optimized at the DFT level of theory, using the B3LYP functional ${ }^{8-9}$ and a standard $6-311+G(d, p)$ basis set in Gaussian $09^{10}$ program package or using PBEh-3c/TZVP(COSMO) level with Turbomole program suite. The calculations were done for the molecules in vacuum. The one-dimensional energy scans along a selected interatomic distance were performed with a smaller 6-31G(d) basis set. The QST3 optimization method ${ }^{11-12}$ was applied in the search for the transition states, that is the structures of the reactants, products, and an estimate of the transition state were used. The vibrational frequencies and free energies were calculated for all of the optimized structures, and the stationary-point character (a minimum or a first-order saddle point) was thus confirmed. The transitions states connect the $\mathrm{vdW}$ structures and the intermediates structure. The connectivity was proved by the optimization starting from the transition structure perturbed in positive and negative directions along the normal mode with negative eigenvalue.

\section{5-1) The hetaryne mechanism}

Table S2. Calculated heat of hydrogenation ( $\mathrm{kcal} / \mathrm{mol})$ of selected pyrimidynes.

\begin{tabular}{c|c|c|c|c} 
Entry & $\mathrm{R}^{1}$ & $\mathrm{R}^{2}$ & $\Delta \mathrm{E}_{\text {calcd }}$ & $\Delta \mathrm{G}_{\text {calcd }}$ \\
\hline 1. & $\mathrm{H}$ & $\mathrm{H}$ & 100.2 & 83.6 \\
2. & $\mathrm{SMe}$ & $\mathrm{H}$ & 101.0 & 84.5 \\
3. & $\mathrm{Cl}$ & $\mathrm{SEt}$ & 102.0 & 85.6 \\
4. & $\mathrm{CN}$ & $\mathrm{H}$ & 103.4 & 86.7 \\
\hline
\end{tabular}

The first step of the supposed hetaryne mechanism is the formation of the hetaryne, which is difficult to model, because an interaction with the base is crucial for the dehydrohalogenation step. An estimation of the pyrimidyne energetics was, however, obtained by comparing calculated free energies of the parent pyrimidine compound with the sum of the hydrogen molecule and the pyrimidyne. The heat of hydrogenation is a well-established means to explore the strains and relative stabilities of alkynes and it has been used recently to predict the likelihood that a given hetaryne can be generated. Using B3LYP level of theory, it was shown that all experimentally confirmed hetarynes had the energy of dehydrogenation between the aryne and its parent heterocycle lower than 115 $\mathrm{kcal} / \mathrm{mol}^{13}$ Our pyrimidyne derivatives have the energy of dehydrogenation close to $100 \mathrm{kcal} / \mathrm{mol}$ (Table S2). The structure and electrostatic potential plots for selected pyrimidynes are depicted in Figure S1. The second step in the supposed hetaryne mechanism is the formation of a product with a new cycle by an iEDDA reaction. Then a nitrogen molecule is expelled from the intermediate yielding the final product. The transition structures for both processes together with the calculated reaction barriers, which were close to $20 \mathrm{kcal} / \mathrm{mol}$ (Figure S2 and Table S3) are also attached.<smiles>c1cncnc1</smiles>
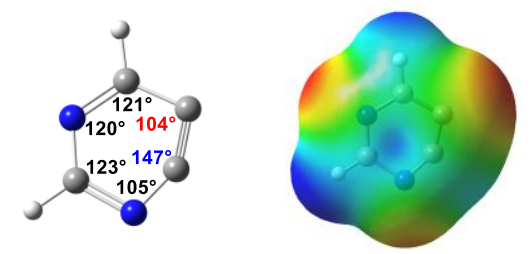

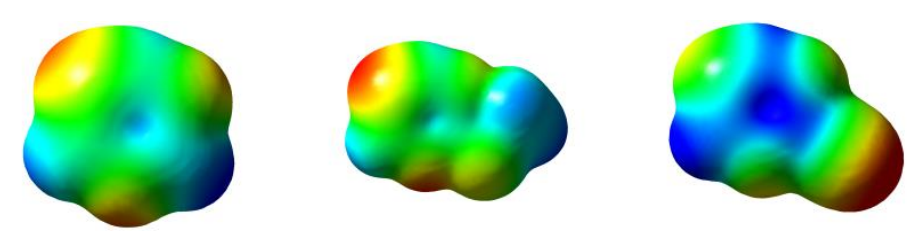<smiles>c1cncnc1</smiles><smiles>CSc1ncccn1</smiles><smiles>N#Cc1ncccn1</smiles>

Figure S1. Valence angles in geometry-optimized pyrimidyne and its electrostatic potential plot. The triple bond in the pyrimidynes is highly polarized with partial negative charge at position 5 of the heterocycle. Electron-donating substituents, such as thiomethyl, increase the polarization, while electron-withdrawing substituents do the opposite.

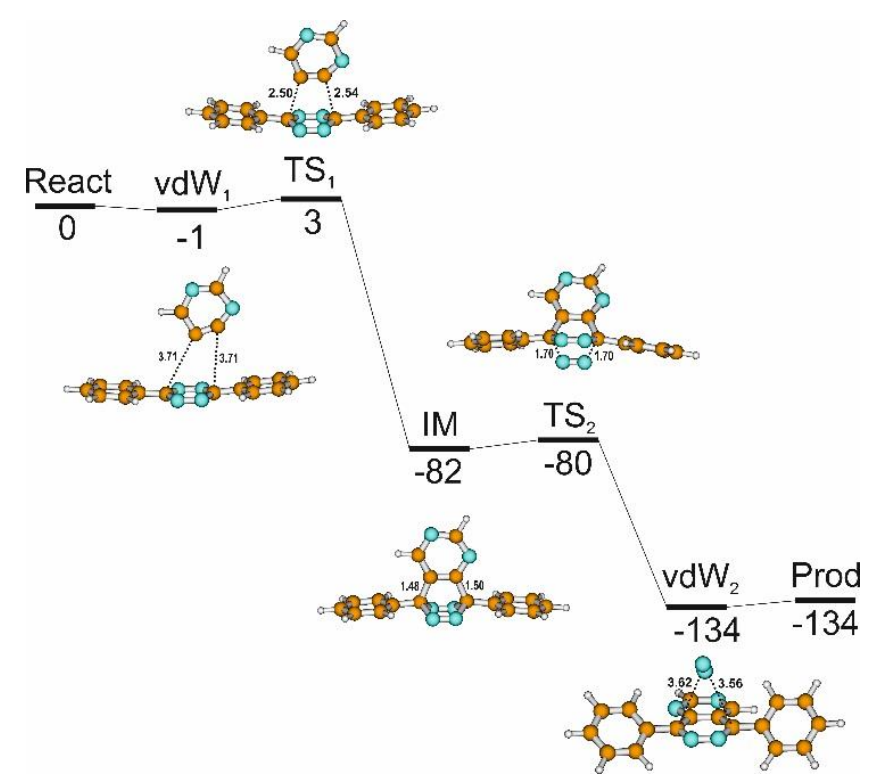

Figure S2. Calculated (PBEO/TZVP) reaction coordinate of the reaction between pyrimidyne and tetrazine. All energies are in $\mathrm{kcal} / \mathrm{mol}$.

Table S3. Calculated barriers of reaction (B3LYP/6-311+g**, electronic and free energies) of pyrimidyne derivatives with diphenyltetrazine.

\begin{tabular}{c|c|c|c|c} 
Entry & $\mathrm{R}^{1}$ & $\mathrm{R}^{2}$ & $\begin{array}{c}\Delta \mathrm{E}^{\#} / \\
\mathrm{kcal} \cdot \mathrm{mol}^{-1}\end{array}$ & $\begin{array}{c}\Delta \mathrm{G}^{\#} / \\
\mathrm{kcal} \cdot \mathrm{mol}^{-1}\end{array}$ \\
\hline 1. & $\mathrm{H}$ & $\mathrm{H}$ & 6.9 & 20.8 \\
2. & $\mathrm{CN}$ & $\mathrm{H}$ & 6.1 & 19.9 \\
3. & $\mathrm{SMe}$ & $\mathrm{H}$ & 7.0 & 20.6 \\
4. & $\mathrm{H}$ & $\mathrm{SEt}$ & 8.3 & 22.4 \\
\hline
\end{tabular}


It is crucial to note that it was recently shown that the degree of regioselectivity one may expect in a reaction involving a hetaryne intermediate is closely related to the distortion of the triple bond and it can be correlated to the difference in bond angles. Differences larger than $4^{\circ}$ suggest synthetically useful regioselectivities. ${ }^{14}$ In our case, DFT calculations revealed enormous angular distortions (Figure 4 in the main text, Table S4) of the pyrimidyne with the difference in bond angles exceeding $40^{\circ}$, which supports the formation of one regioisomer only.

Table S4. Calculated valence angles (degree) of the triple bond in pyrimidyne derivatives

\begin{tabular}{ccccc}
\hline & $\mathrm{R}^{1}$ & $\mathrm{R}^{2}$ & \multicolumn{2}{c}{ B3LYP/6-311+g** } \\
\cline { 3 - 5 } & & & N1-C6-C5 & C6-C5-C4 \\
\cline { 3 - 5 } & $\mathrm{H}$ & $\mathrm{H}$ & 146.6 & 104.2 \\
& $\mathrm{Cl}$ & $\mathrm{SEt}$ & 147.8 & 103.2 \\
& $\mathrm{CN}$ & 146.3 & 104.7 \\
\hline
\end{tabular}

\section{5-2) The ionic mechanism}

The calculations of electrostatic potentials of tetrazine $\mathbf{S 1 8}$ revealed that the tetrazine carbon atom that is attached to the morpholine substituent is the more positive one and hence attacked by the pyrimidine anion.
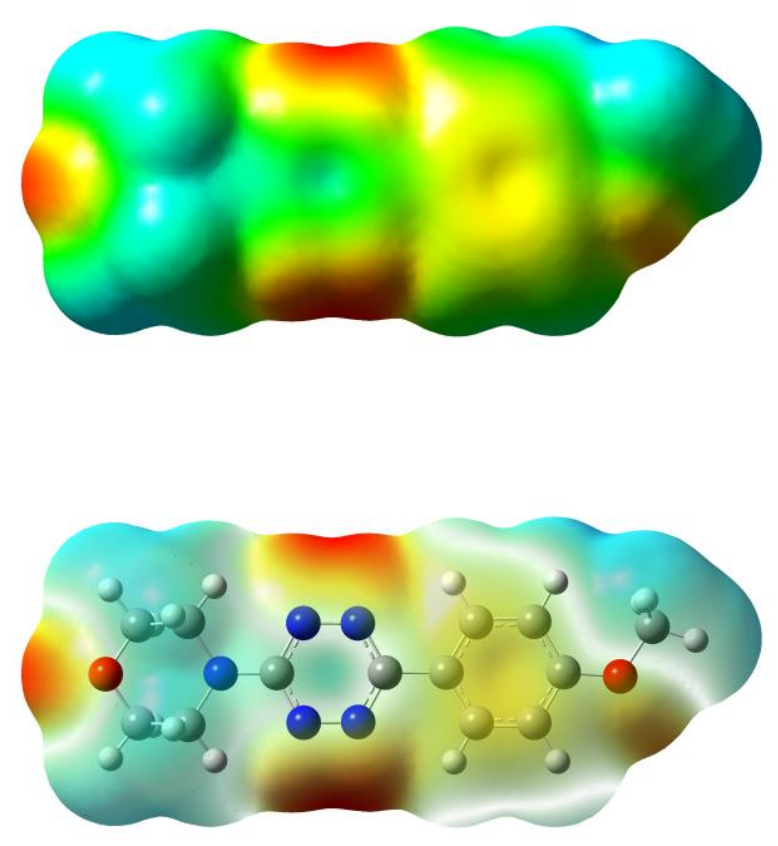
The cyclization step was also evaluated by a one-dimensional energy scan, which showed a modest energy barrier of the reaction of ca $28 \mathrm{kcal} / \mathrm{mol}$. The transition state structure was then optimized and its connectivity with the intermediate and the product was proved by the optimization starting from the transition structure perturbed in positive and negative directions along the normal mode with negative eigenvalue. The geometry optimization on the side of the product led to simultaneous loss of fluoride anion and nitrogen molecule yielding directly the bicyclic final product and an overall energy gain of $52 \mathrm{kcal} / \mathrm{mol}$ (with respect to the starting anion and tetrazine). The overall calculated reaction coordinate is shown in Figure S3. An alternative attack of the negatively charged carbon on C6 of the pyrimidine would explain the formation of the spiro compound $\mathbf{1 0}$ and $\mathbf{1 6 .}$ 


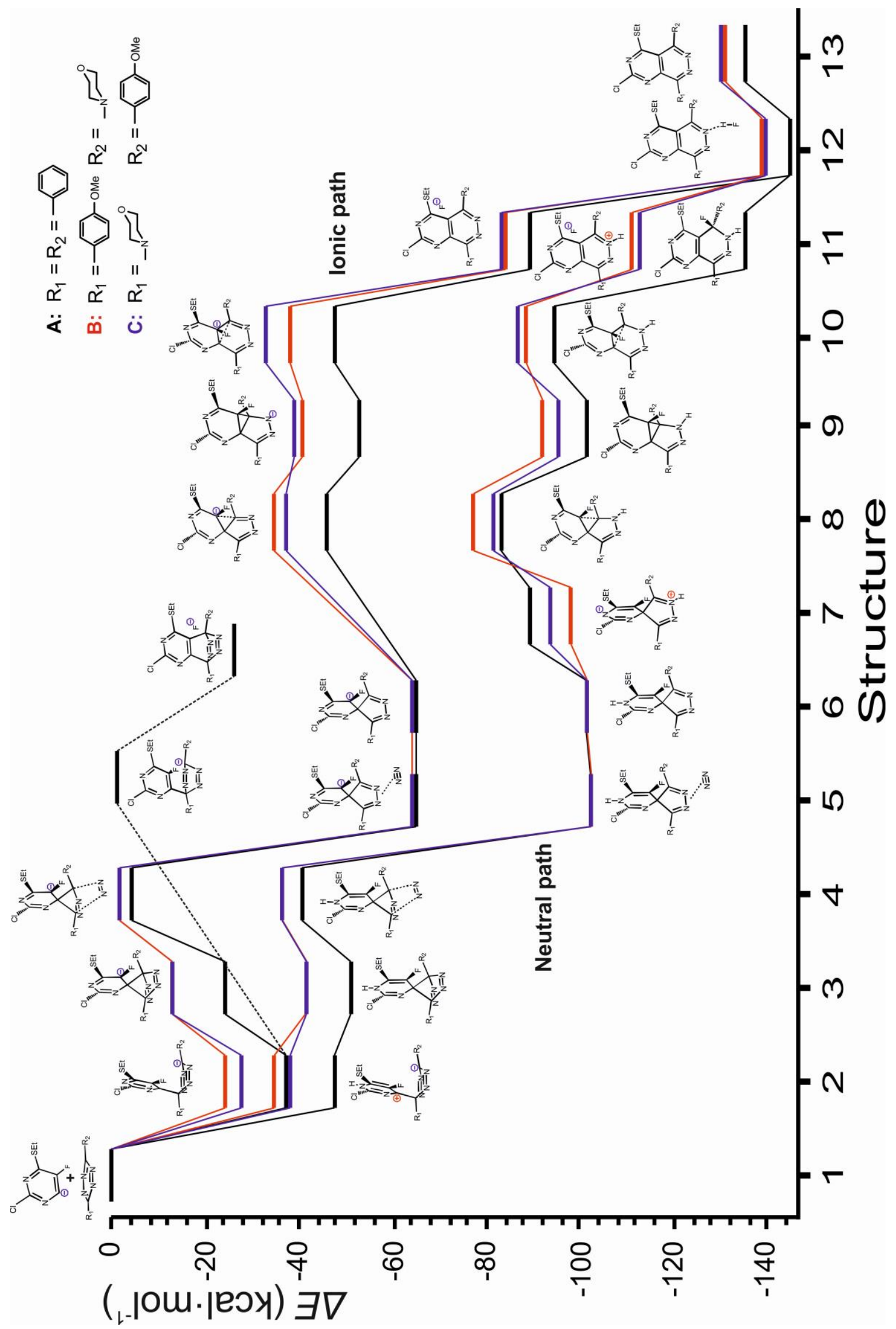

Figure S3. Calculated (PBEh-3c/TZVP(COSMO) ${ }^{15}$ ) reaction coordinate of the reaction between 5fluoropyrimidine anion and tetrazine. 
Reaction of isopropyl-phenyl-tetrazine $\mathbf{S 1 0}$ with 5-fluoropyrimidine $\mathbf{S 4}$ and with 6-chloropyrimidine S8

Since the reaction of the pyrimidine anion with the tetrazine is a downhill reaction, the regioselectivity is driven by the energy of the resulting intermediate. In both cases, lower energy was found for the intermediate that leads after cyclization to the experimentally observed product $\mathbf{6 g}$.<smiles>CCSc1nc(Cl)nc(C2(C(C)C)N=NC(c3ccccc3)=NN2)c1F</smiles>

$17 \mathrm{kcal} / \mathrm{mol}$<smiles>CCSc1nc(Cl)nc(Cl)c1C1(c2ccccc2)N=NC(C(C)C)=NN1</smiles>

$5 \mathrm{kcal} / \mathrm{mol}$<smiles>CCSc1nc(Cl)nc(C2(c3ccccc3)N=NC(C(C)C)=NN2O)c1F</smiles>

$0 \mathrm{kcal} / \mathrm{mol}$<smiles>CCSc1nc(Cl)nc(Cl)c1C1(C(C)C)N=NC(c2ccccc2)=NN1</smiles><smiles>CCC</smiles><smiles>CCCCC</smiles><smiles>CCSc1nc(I)nc2c(-c3ccccc3)nnc(C(C)C)c12</smiles>

$6 \mathrm{~g}$

Figure S4. The structures and relative free energies (B3LYP/6-31 $\mathrm{g}^{*}$ ) of intermediates of the reaction of halopyrimidines with tetrazine $\mathbf{S 1 0 .}$

\section{References}

1. Zhu, J.; Hiltz, J; Lennox, R. B.; Schirrmacher, R. Chem. Comm. 2013, 49, 10275-10277.

2. Novák, Z.; Bostai, B.; Csékei, M.; Lörincz, K.; Kotschy, A. Heterocycles 2003, 60, 2653-2668.

3. Audebert, P.; Miomandre, F.; Clavier, G.; Vernières, M.-C.; Badré, S.; Méallet-Renault, R. Chem. Eur. J. 2005, 11, 5667-5673.

4. Boger, D. L.; Coleman, R. S.; Panek, J. S., Huber, F. X.; Sauer, J.; J. Org. Chem. 1985, 50, 5377-5379.

5. Liu, H.; Wei, Y. Tetrahedon Lett. 2013, 54, 4645-4648.

6. Wang, D.; Chen, W.; Zheng, Y.; Dai, C.; Wang, K.; Ke, B.; Wang, B. Org. Biomol. Chem. 2014, 12, 3950.

7. Leconte, N.; Keromnes-Wuillame, A.; Suzenet, F.; Guillaumet, G. Synlett 2007, 204-210.

8. Becke, A. D. J. Chem. Phys. 1993, 98, 5648-5652.

9. Lee, C. T.; Yang, W. T.; Parr, R. G. Phys. Rev. B 1988, 37, 785-789.

10. Frisch, M. J.; Trucks, G. W.; Schlegel, H. B.; Scuseria, G. E.; Robb, M. A.; Cheeseman, J. R.; Scalmani, G.; Barone, V.; Mennucci, B.; Petersson, G. A.; Nakatsuji, H.; Caricato, X.; Li, X.; Hratchian, H. P.; Izmaylov, A. F.; Bloino, J.; Zheng, G.; Sonnenberg, J. L.; Hada, M.; Ehara, M.; Toyota, K.; Fukuda, R.; Hasegawa, J.; Ishida, M.; Nakajima, T.; Honda, Y.; Kitao, O.; Nakai, H.; Vreven, T.; Montgomery, J., J. A.; Peralta, J. E.; Ogliaro, F.; Bearpark, M.; Heyd, J. J.; Brothers, E.; Kudin, K. N.; Staroverov, V. N.; Kobayashi, R.; Normand, J.; Raghavachari, K.; Rendell, A.; Burant, J. C.; Iyengar, S. S.; Tomasi, J.; Cossi, M.; Rega, N.; Millam, J. M.; Klene, M.; Knox, J. E.; Cross, J. B.; Bakken, V.; Adamo, C.; Jaramillo, J.; Gomperts, R.; Stratmann, R. E.; Yazyev, O.; Austin, A. J.; Cammi, R.; Pomelli, C.; Ochterski, J. W.; Martin, R. L.; Morokuma, K.; Zakrzewski, V. G.; Voth, G. A.; Salvador, P.; Dannenberg, J. J.; Dapprich, S.; Daniels, A. D.; Farkas, O.; Foresman, J. B.; Ortiz, J. V.; Cioslowski, J.; Fox, D. J. Gaussian 09, Revision A.02, Gaussian, Inc.: Wallingford CT, 2009.

11. Peng, C.; Ayala, P. Y.; Schlegel, H. B.; Frisch, M. J. J. Comput. Chem. 1996, 17, 49-56.

12. Peng, C.; Schlegel, H. B. Israel J. Chem. 1994, 33, 449-454.

13. Goetz, A. E.; Bronner, S. M.; Cisneros, J. D.; Melamed, J. M.; Paton, R. S.; Houk, K. N.; Garg, N. K. Angew. Chem., Int. Ed. 2012, 51 , 2758. 14. (a) Cheong, P. H. Y.; Paton, R. S.; Bronner, S. M.; Im, G. Y. J.; Garg, N. K.; Houk, K. N. J. Am. Chem. Soc. 2010, 132, 1267. (b) Im, G. Y. J.; Bronner, S. M.; Goetz, A. E.; Paton, R. S.; Cheong, P. H. Y.; Houk, K. N.; Garg, N. K. J. Am. Chem. Soc. 2010, $132,17933$.

15. Grimme, S.; Antony, J.; Ehrlich, S.; Krieg, H. J. Chem. Phys. 2010, 132, 154104. 
6) ${ }^{1} \mathrm{H}$ and ${ }^{13} \mathrm{C}$ NMR spectra of fluoropyrimidine $\mathbf{S 4}$

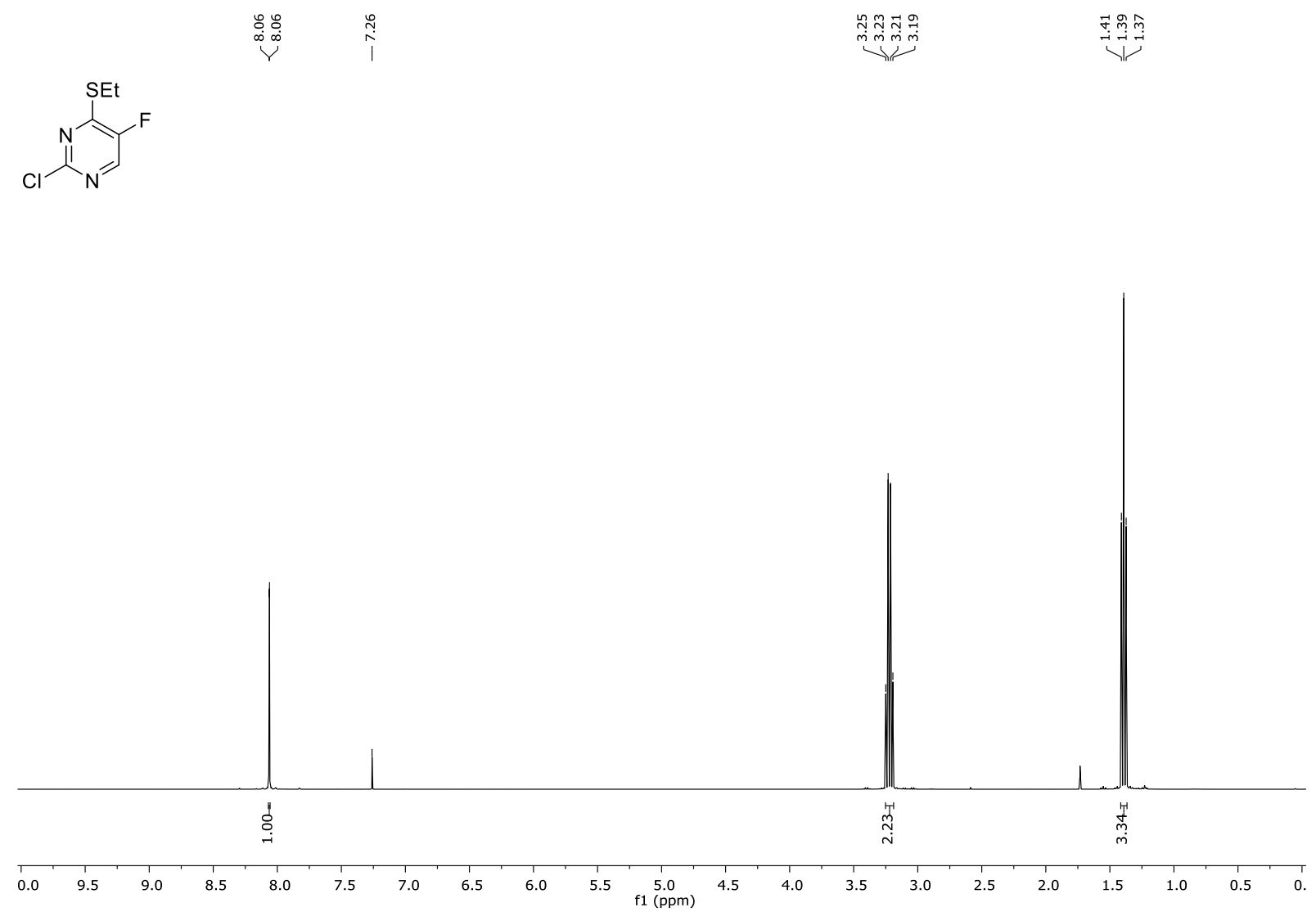

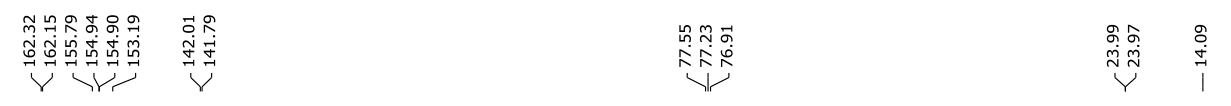

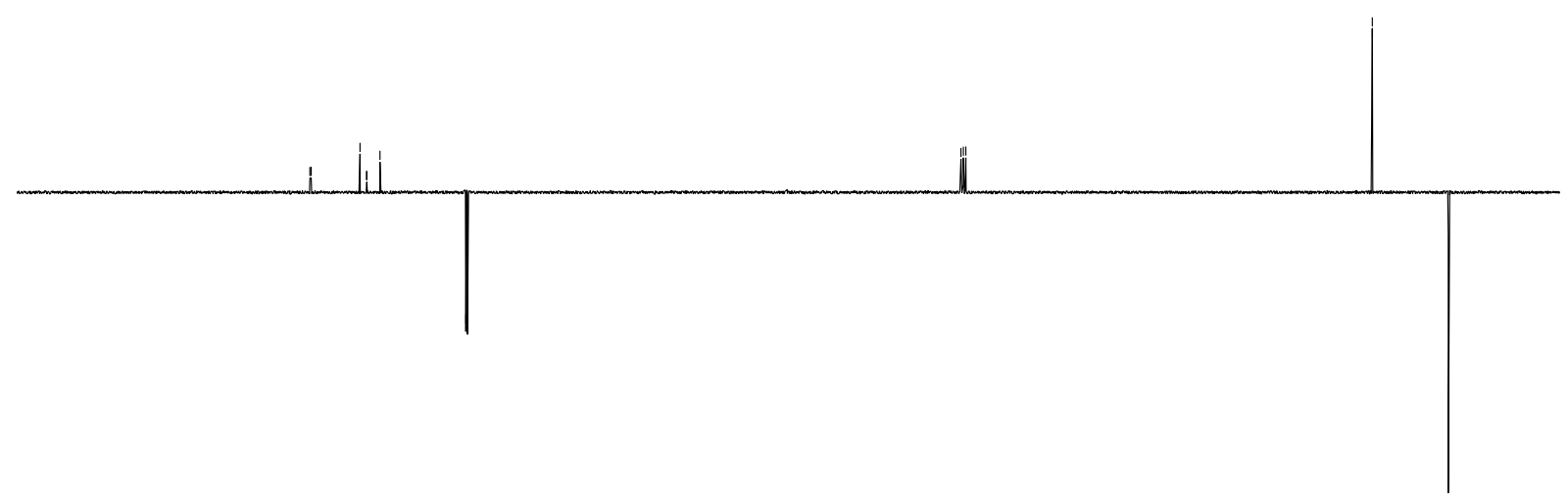

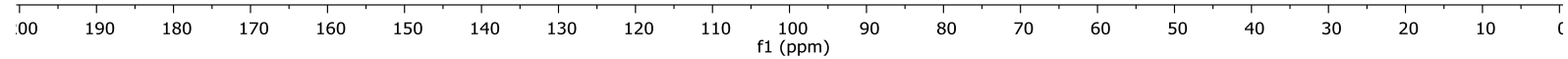


${ }^{1} \mathrm{H}$ and ${ }^{13} \mathrm{C}$ NMR spectra of chloropyrimidine $\mathbf{S 5}$

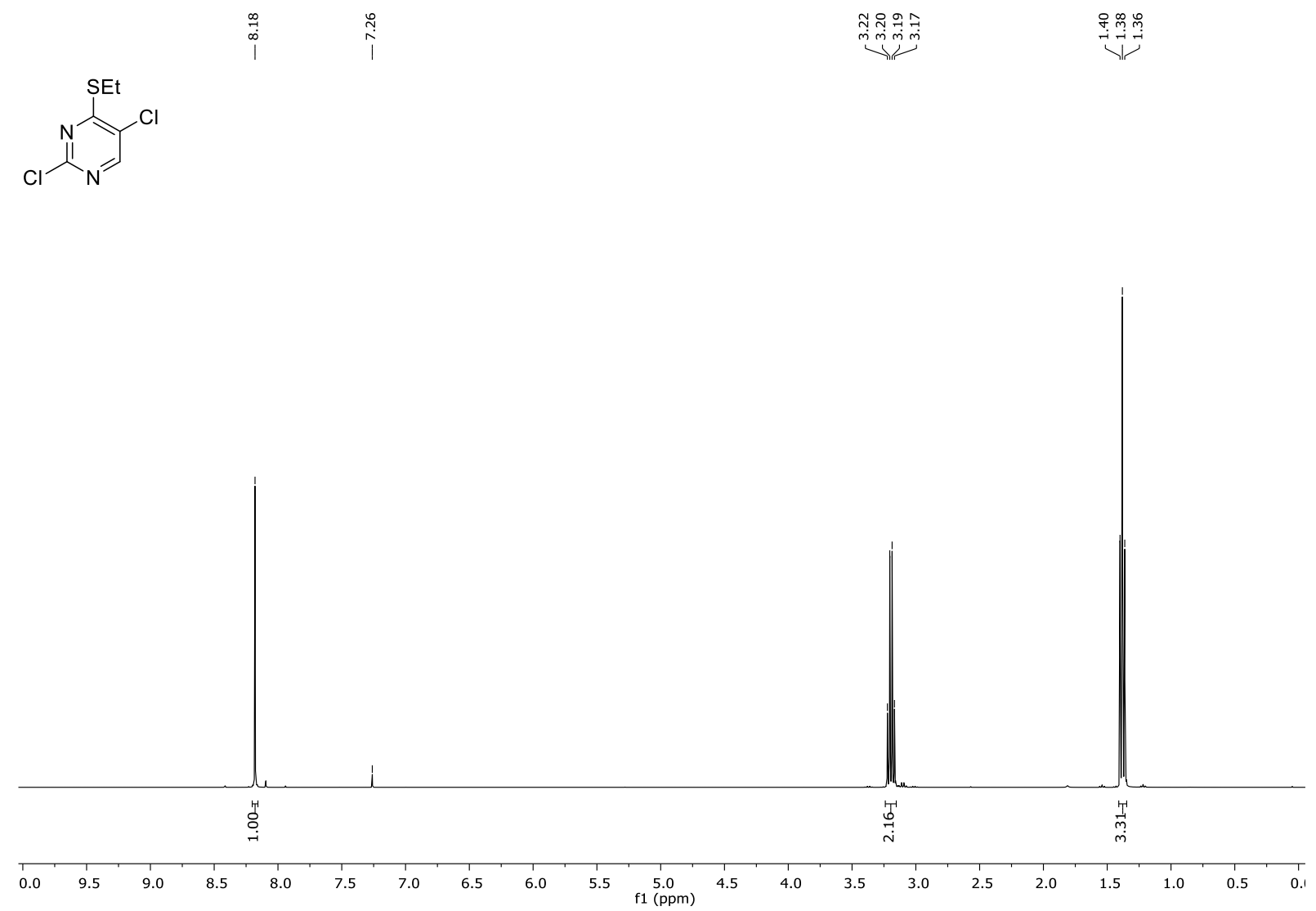

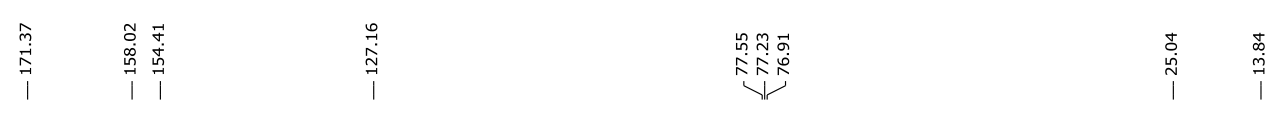

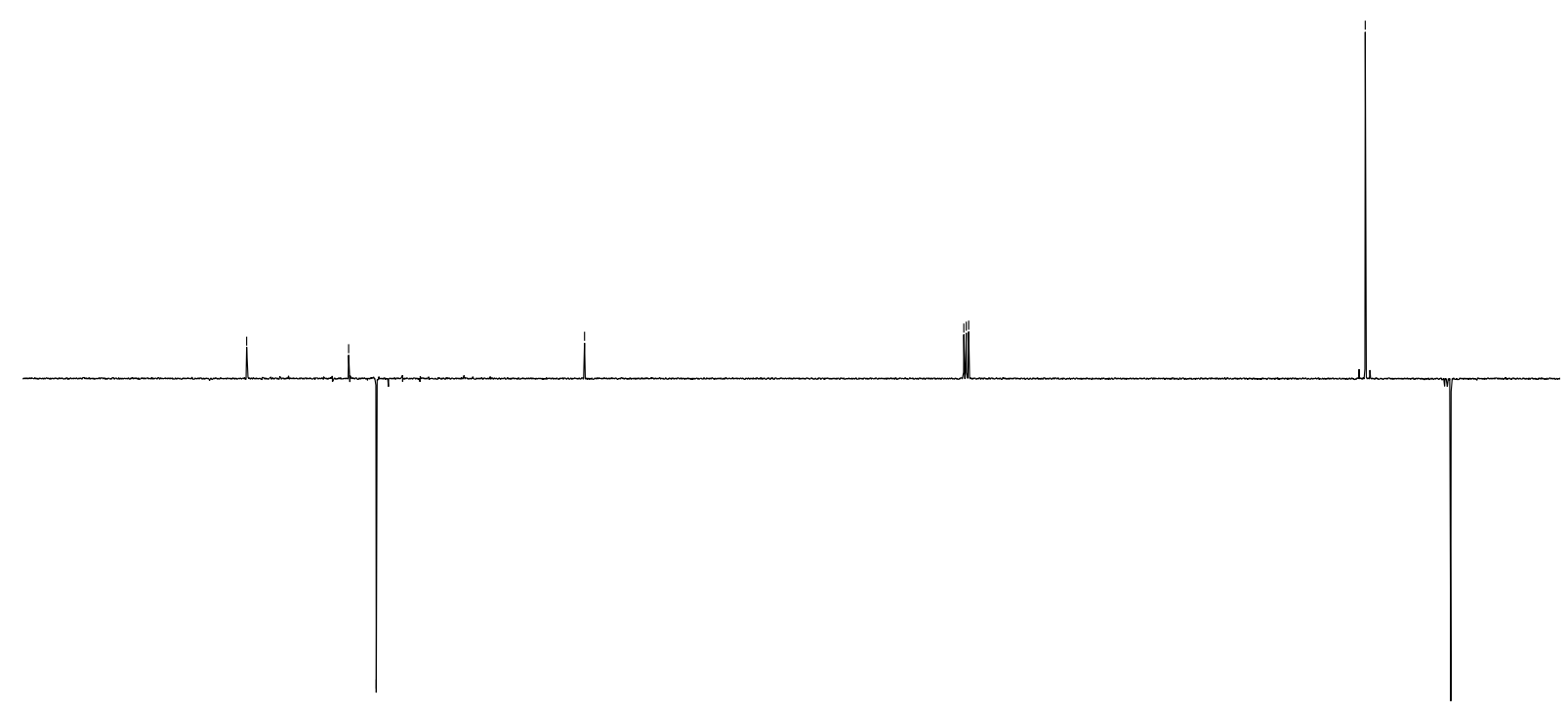

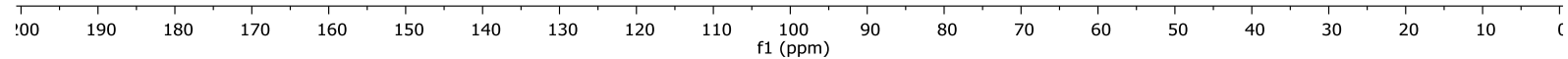


${ }^{1} \mathrm{H}$ and ${ }^{13} \mathrm{C}$ NMR spectra of bromopyrimidine $\mathbf{S 6}$
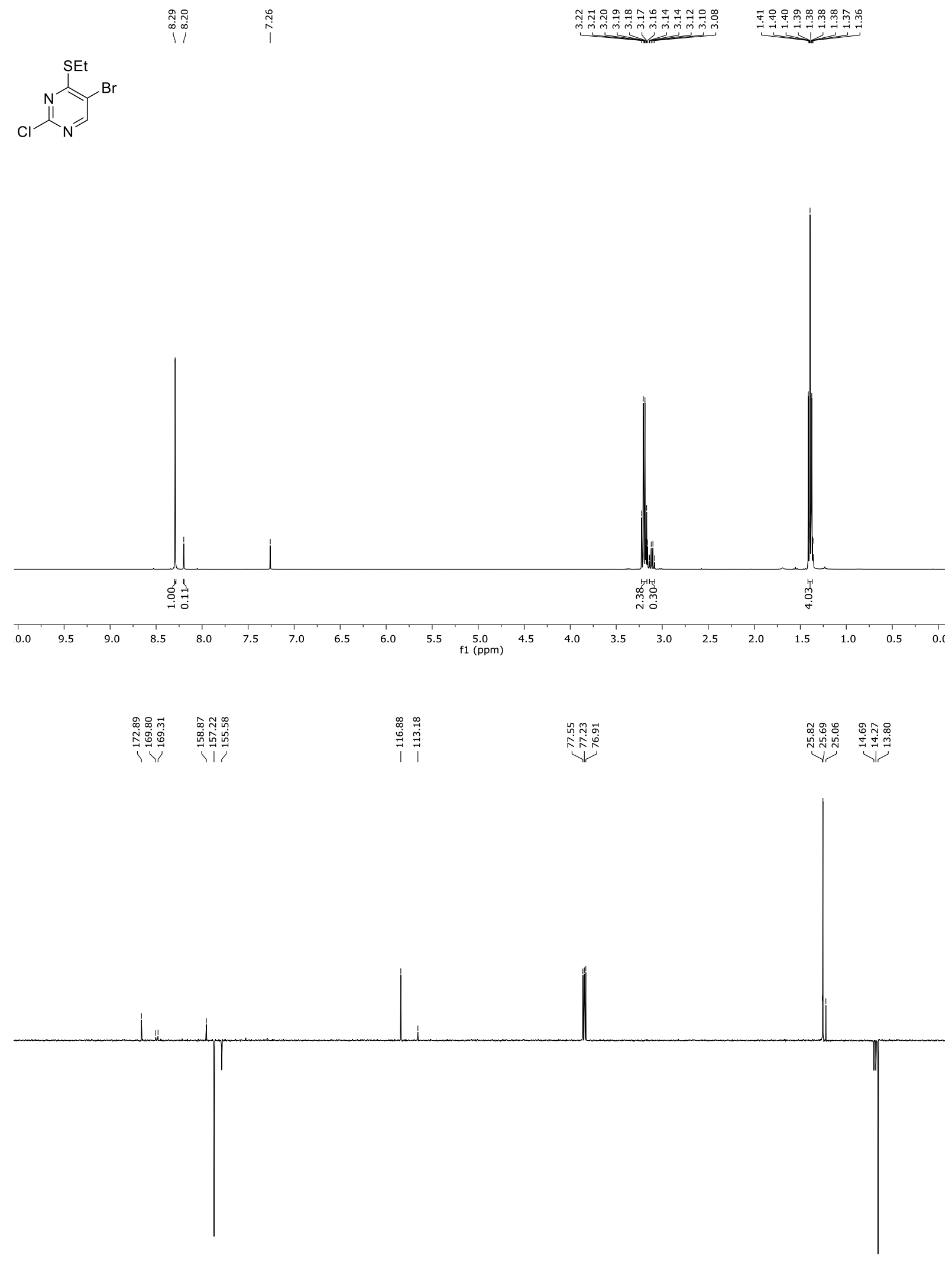

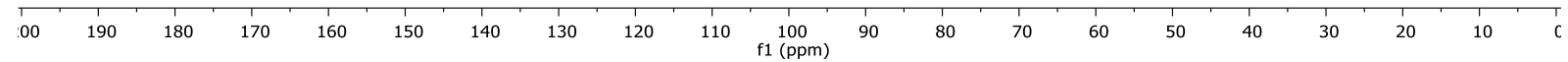


${ }^{1} \mathrm{H}$ and ${ }^{13} \mathrm{C}$ NMR spectra of pyrimidine $\mathbf{S 8}$

$\stackrel{\substack{i \\ i}}{i}$

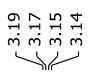

$\overbrace{\mathrm{Cl}}^{\mathrm{SEt}}$

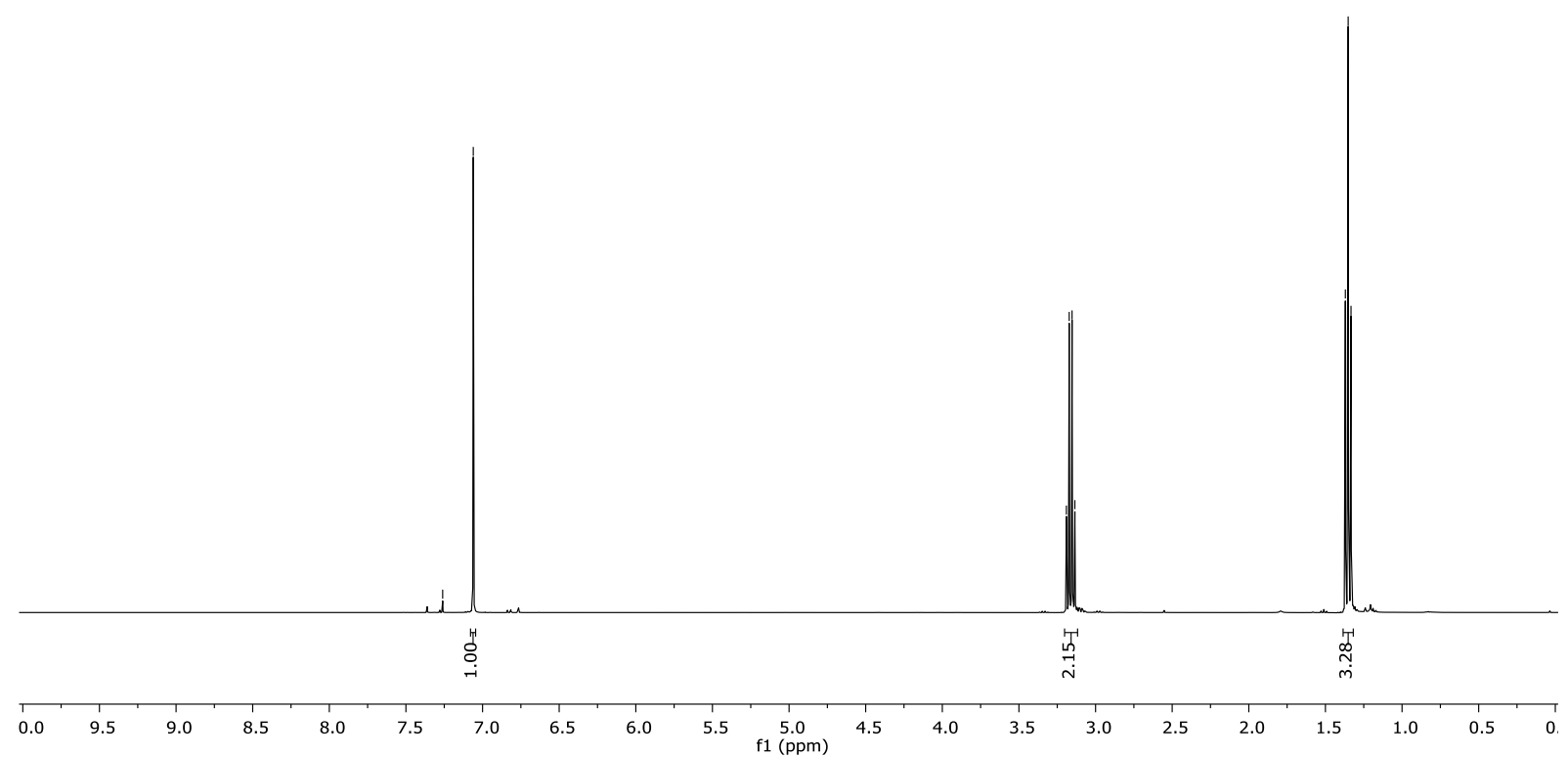

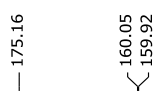

总

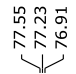

$\underset{\substack{1 \\ \stackrel{d}{+}}}{\stackrel{1}{+}}$

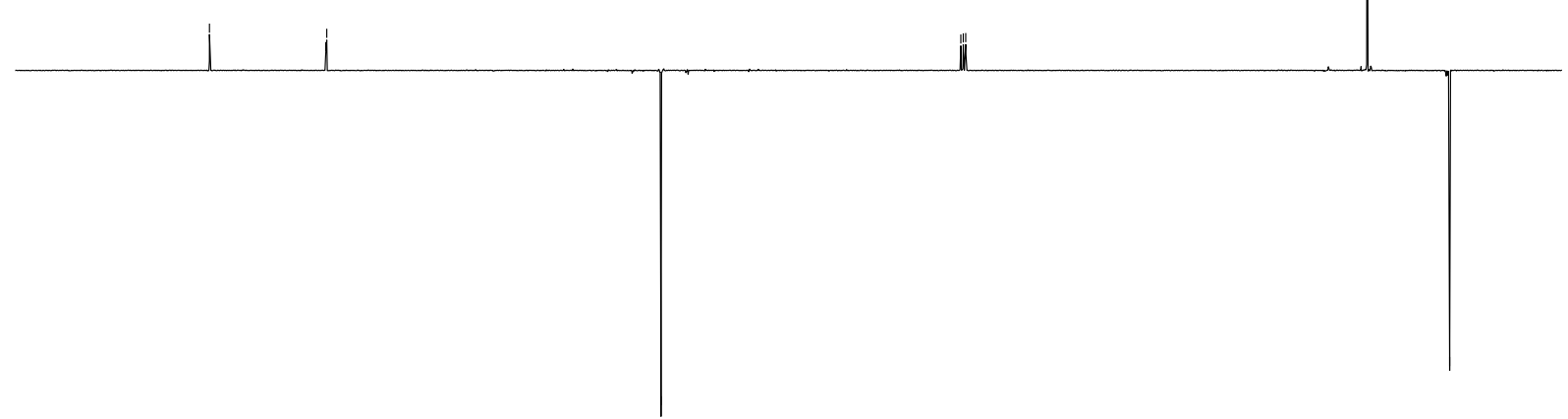

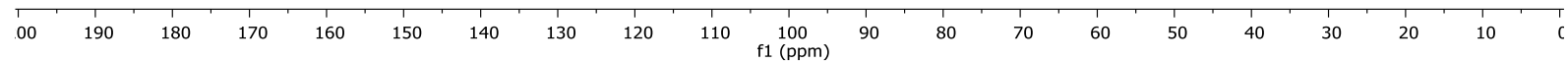


${ }^{1} \mathrm{H}$ and ${ }^{13} \mathrm{C}$ NMR spectra of pyrimidine $\mathbf{S 9}$
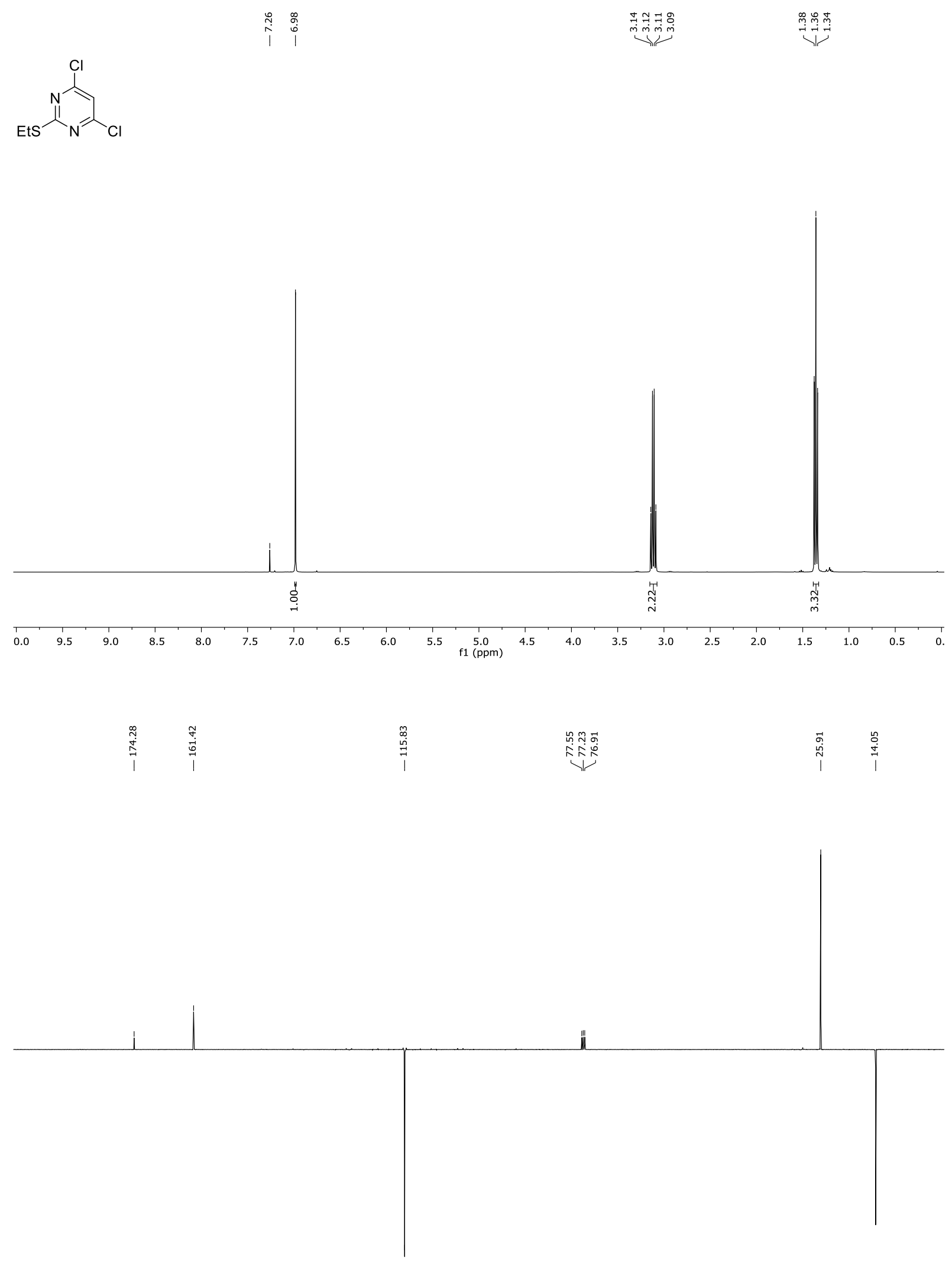

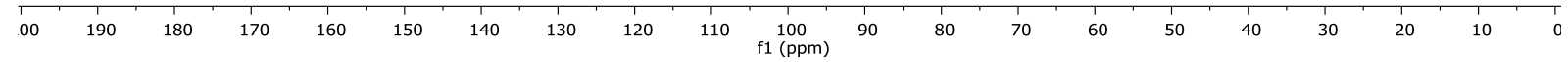


${ }^{1} \mathrm{H}$ and ${ }^{13} \mathrm{C}$ NMR spectra of tetrazine $\mathbf{S 1 0}$

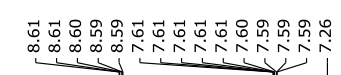

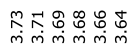

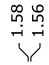
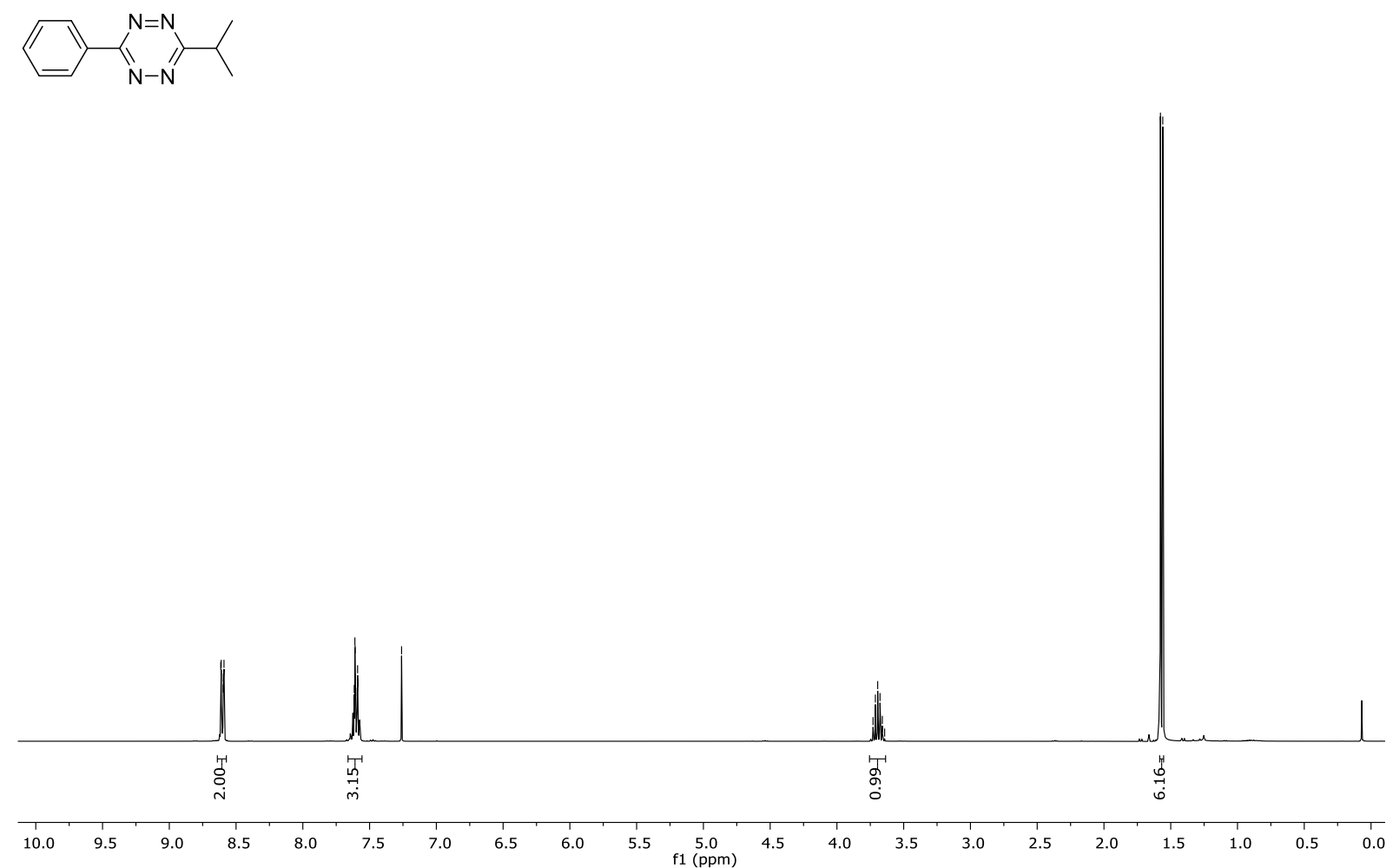

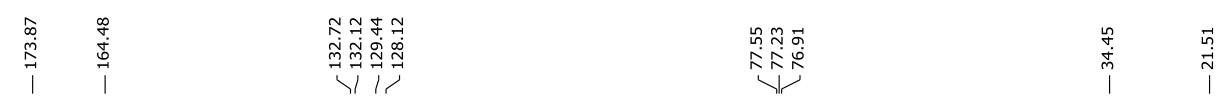

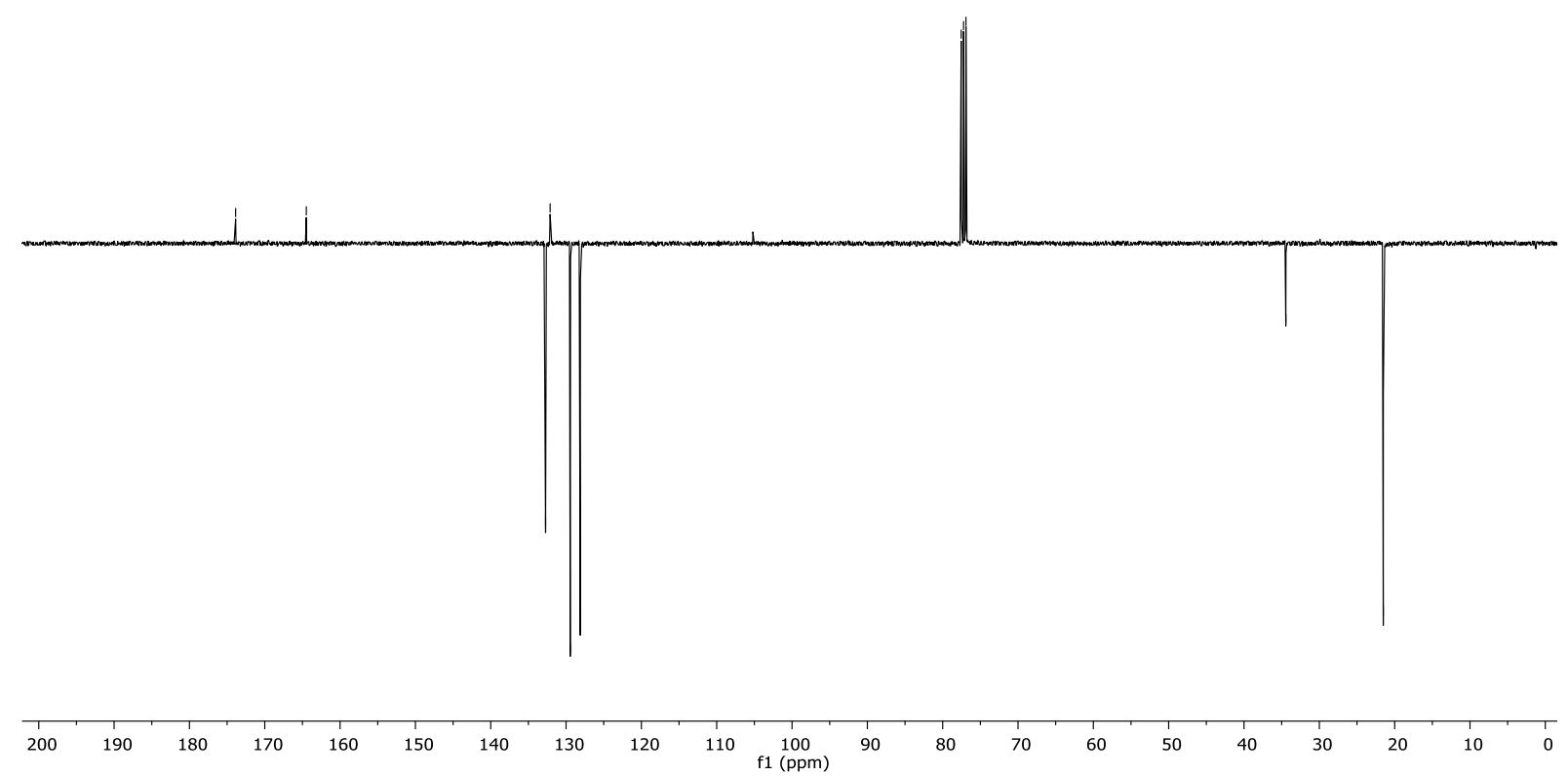


${ }^{1} \mathrm{H}$ and ${ }^{13} \mathrm{C}$ NMR spectra of tetrazine $\mathbf{S 1 2}$
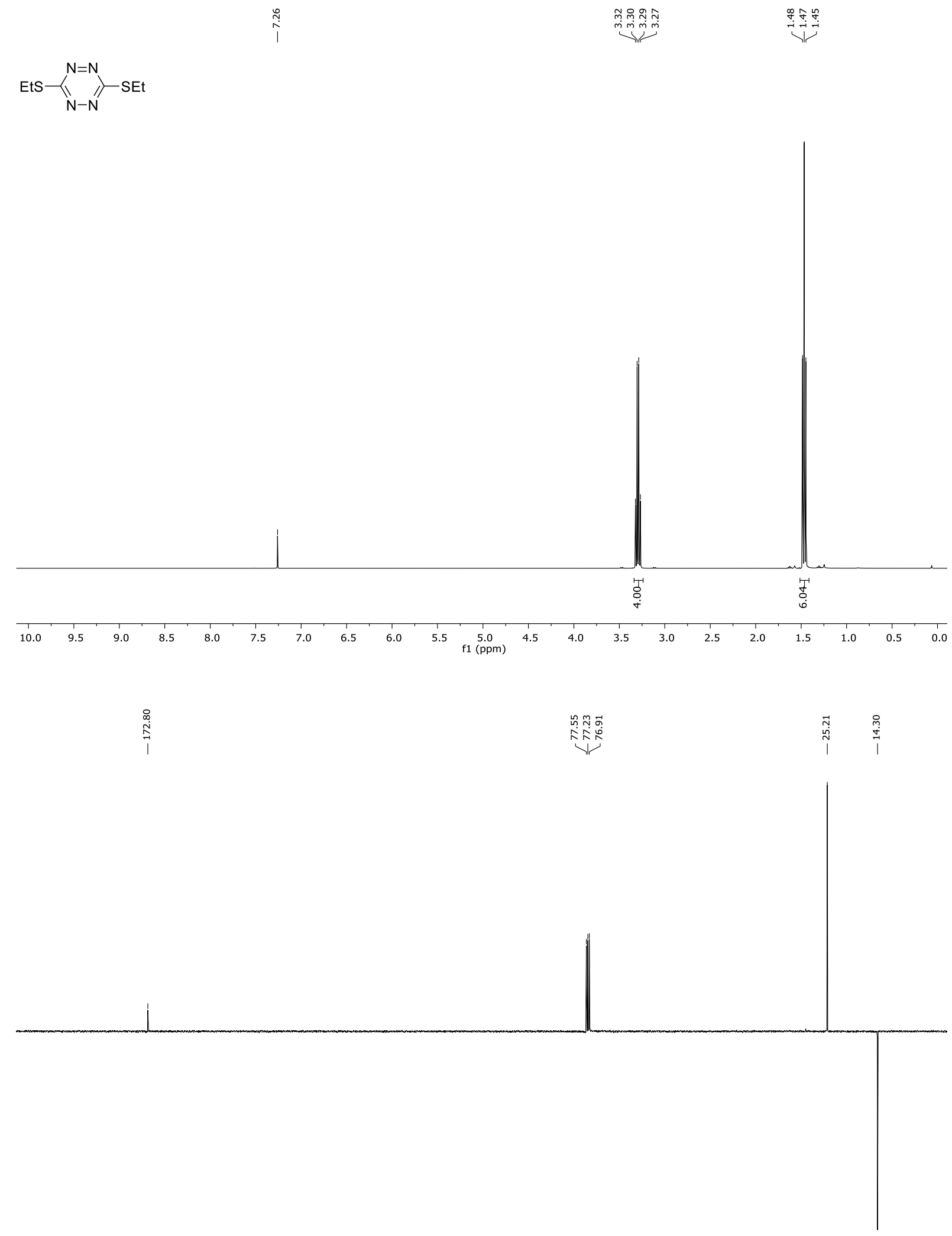

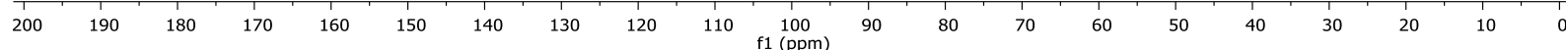


${ }^{1}$ H NMR spectrum of tetrazine $\mathbf{S 1 3}$
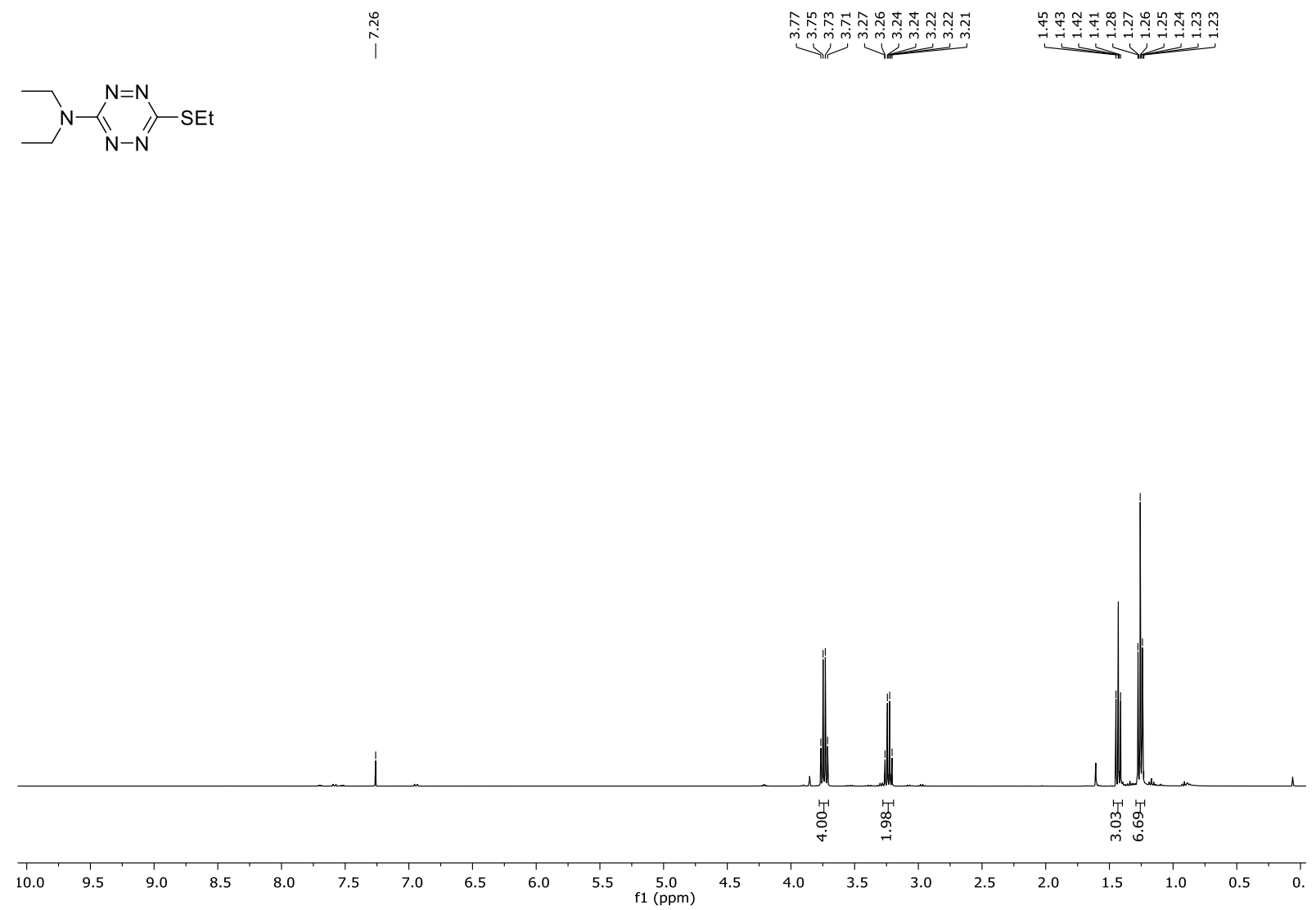

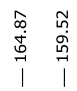

\begin{tabular}{|c|c|c|}
\hline 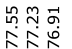 & 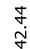 & 空 \\
\hline 4 & I & | \\
\hline
\end{tabular}

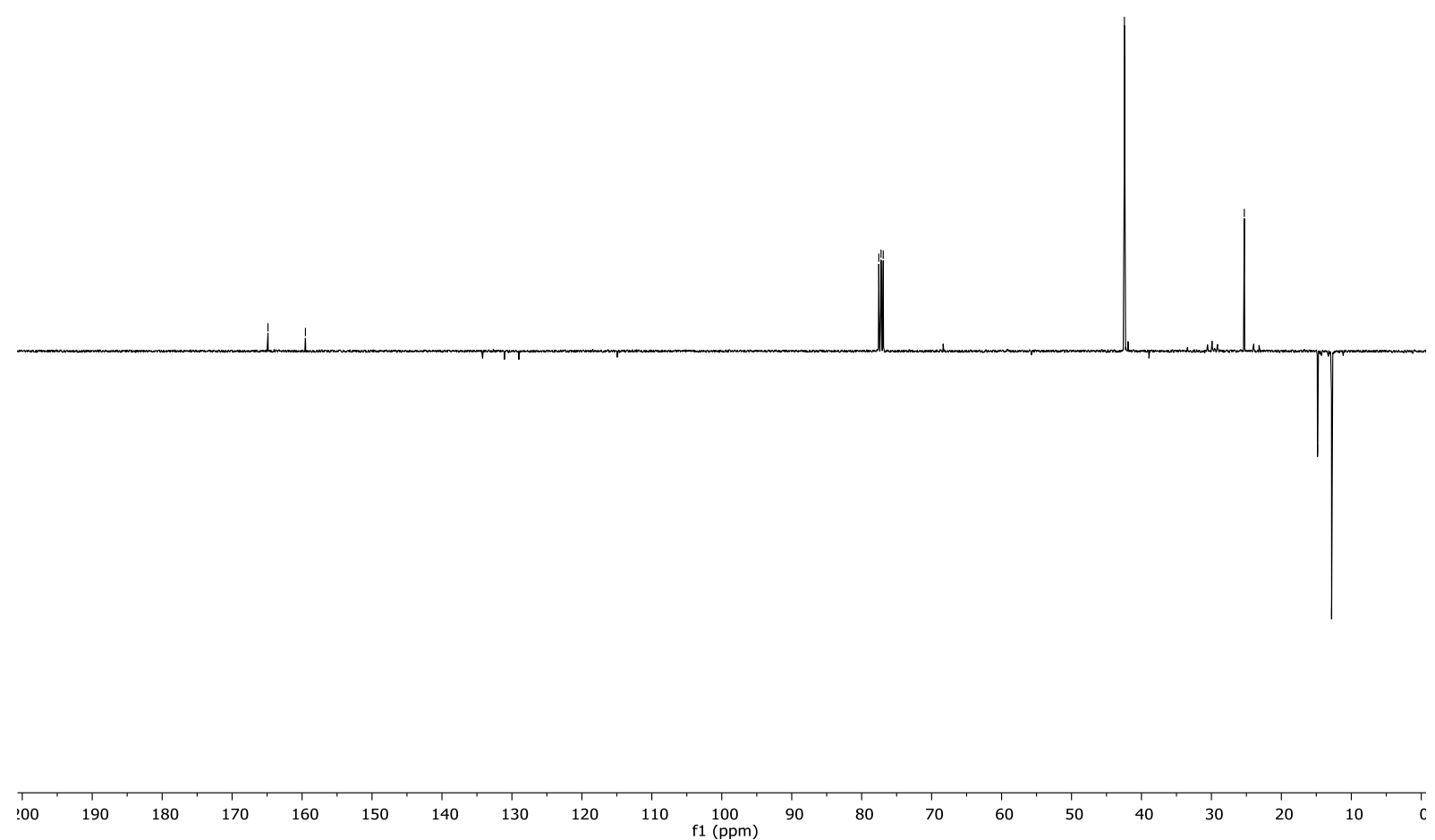


${ }^{1} \mathrm{H}$ and ${ }^{13} \mathrm{C}$ NMR spectra of tetrazine $\mathbf{S 1 4}$
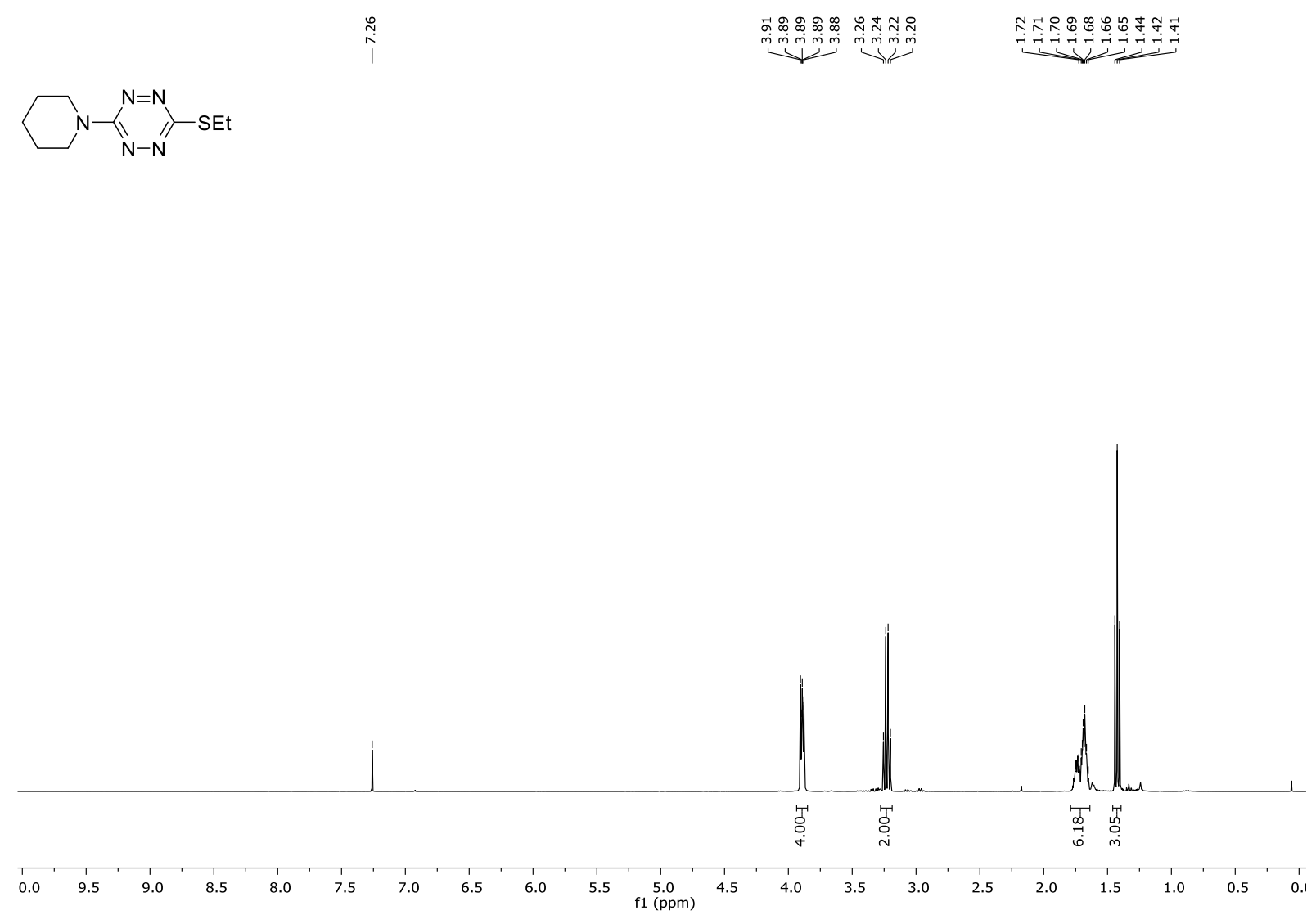

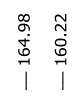

\begin{tabular}{|c|c|c|}
\hline 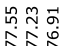 & 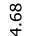 & 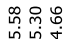 \\
\hline 证 & a & ป \\
\hline
\end{tabular}

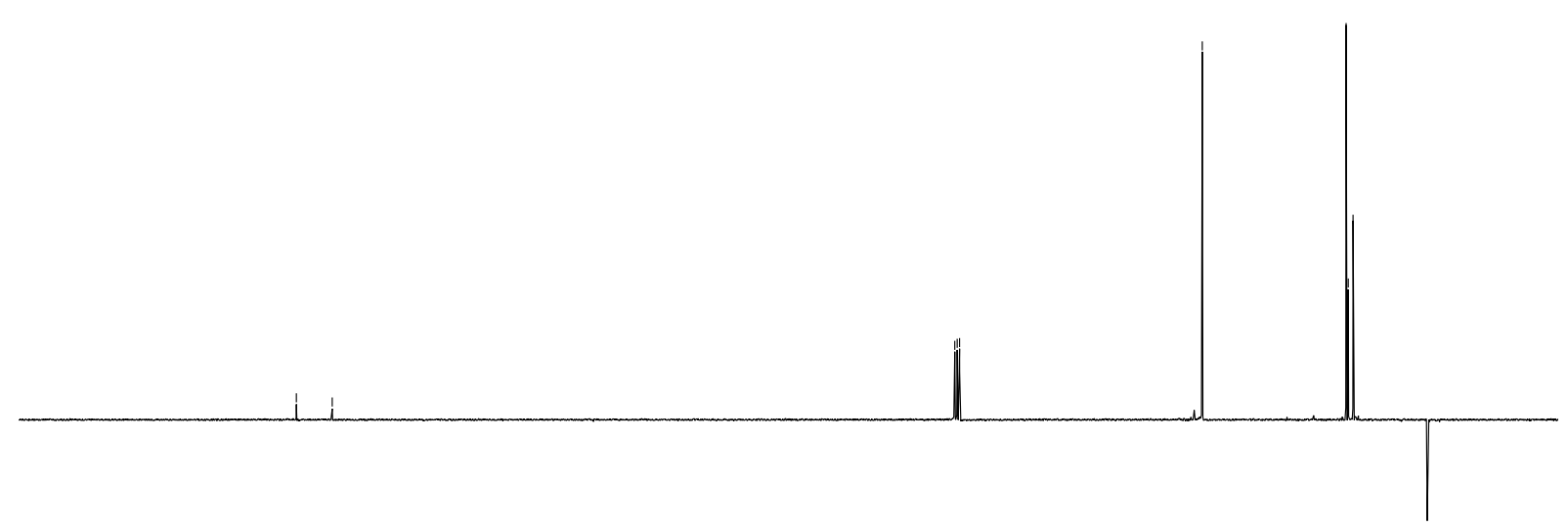

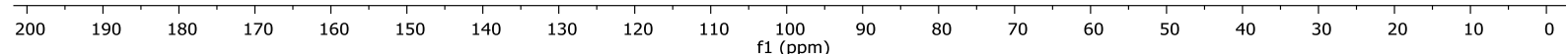


${ }^{1} \mathrm{H}$ and ${ }^{13} \mathrm{C}$ NMR spectra of tetrazine $\mathbf{S 1 5}$

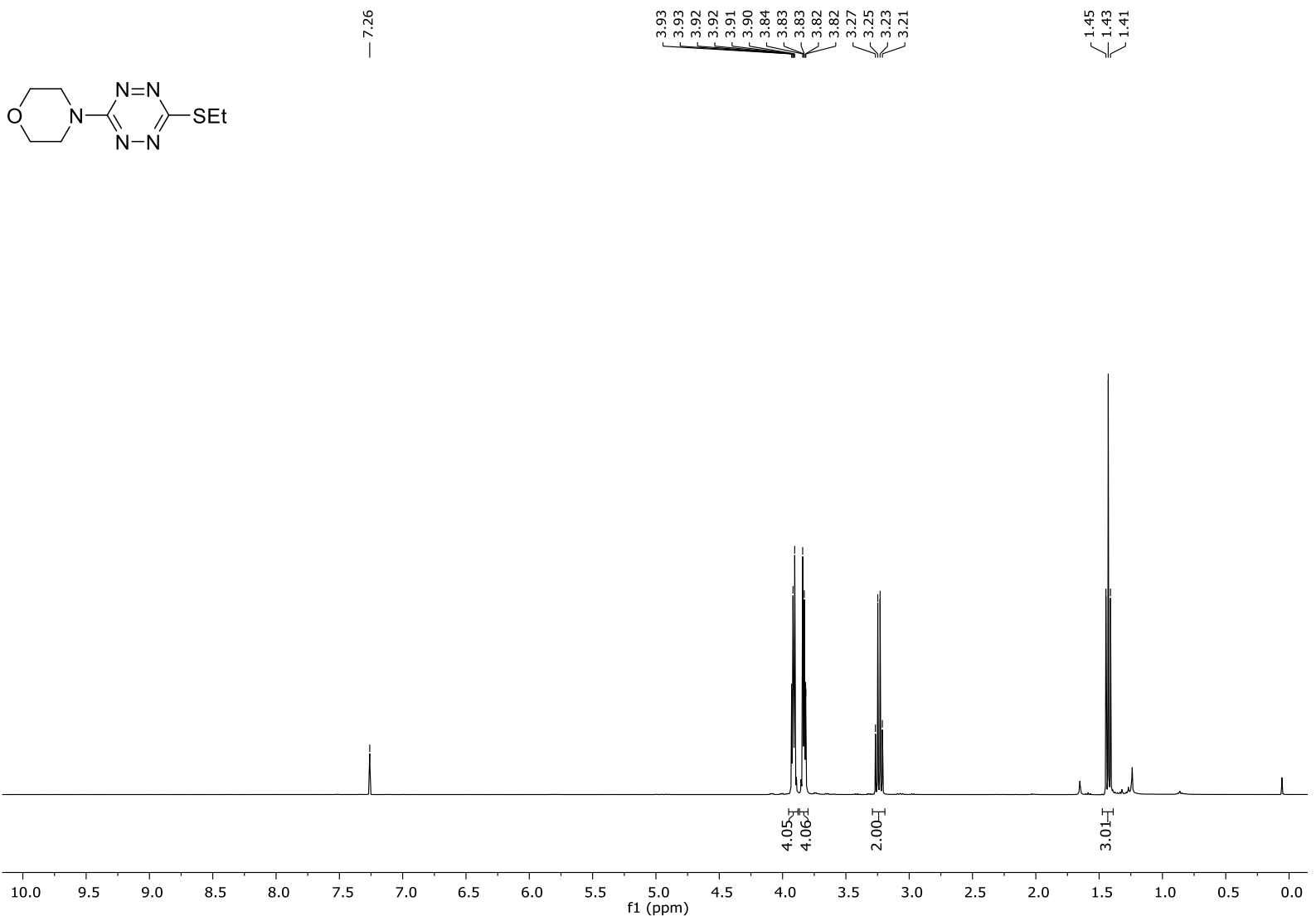

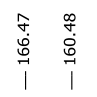

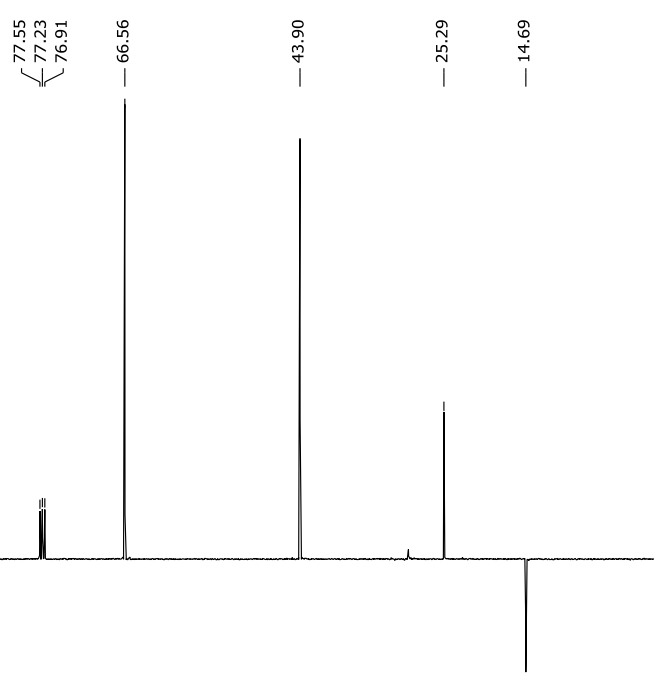

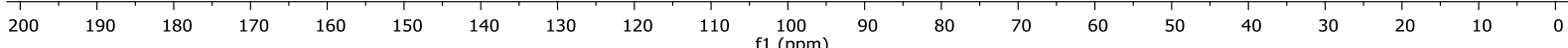


${ }^{1} \mathrm{H}$ and ${ }^{13} \mathrm{C}$ NMR spectra of tetrazine $\mathbf{S 1 6}$
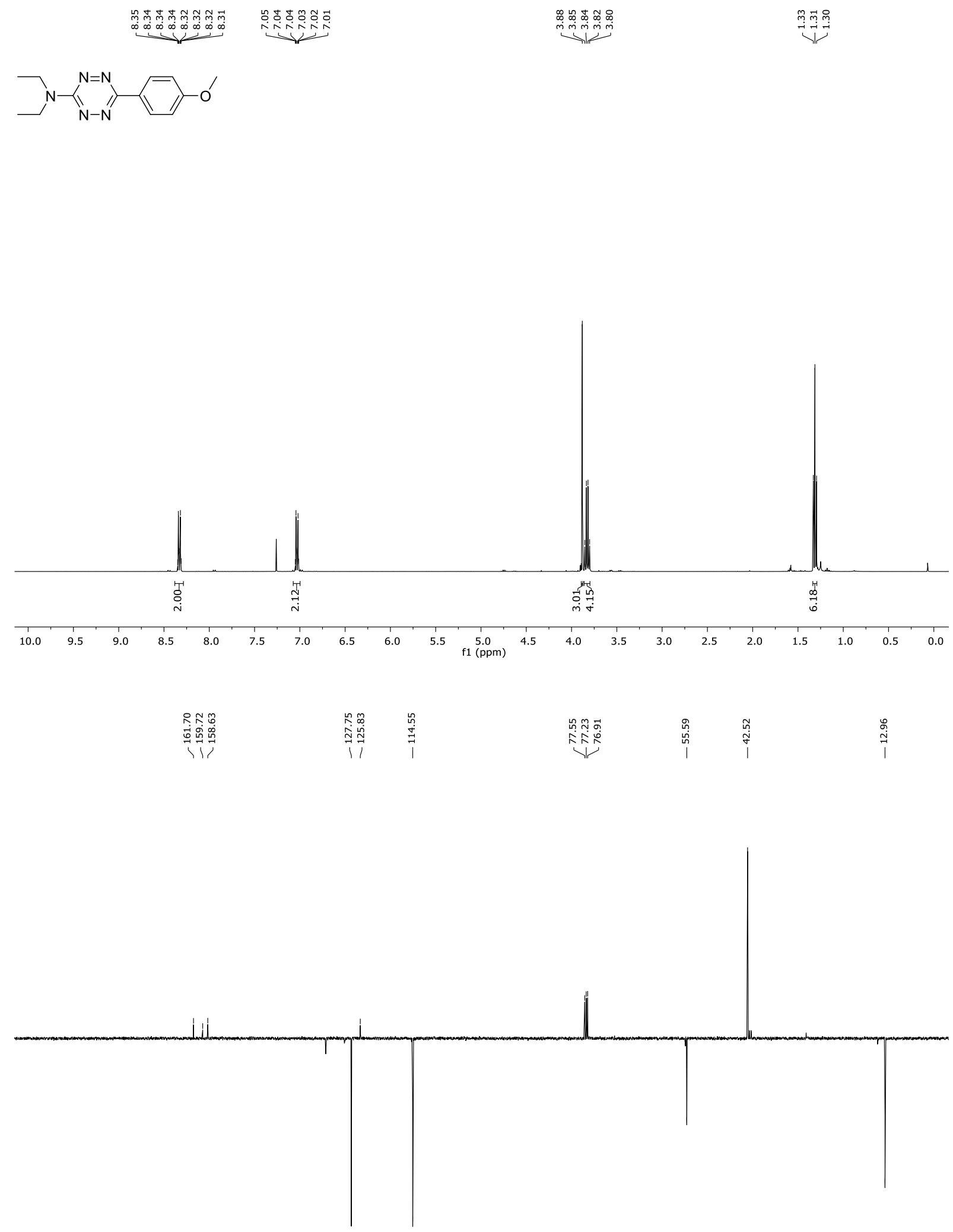

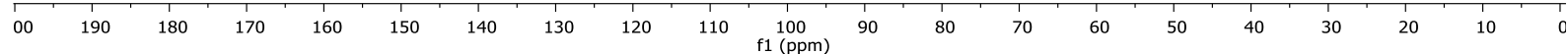


${ }^{1} \mathrm{H}$ and ${ }^{13} \mathrm{C}$ NMR spectra of tetrazine $\mathbf{S 1 7}$
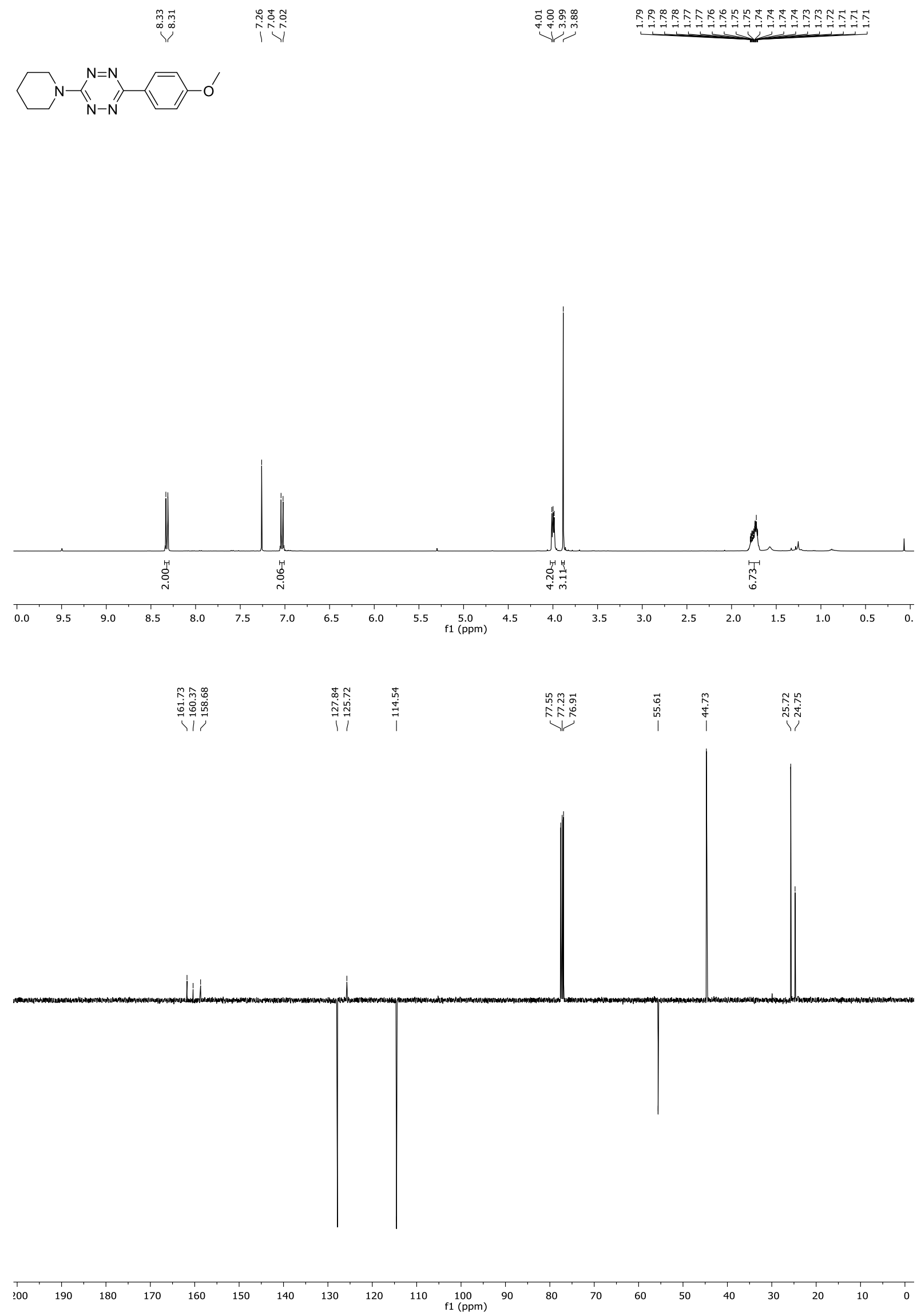
${ }^{1} \mathrm{H}$ and ${ }^{13} \mathrm{C}$ NMR spectra of tetrazine $\mathbf{S 1 9}$

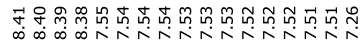

$\underbrace{\infty}{ }^{\infty}$

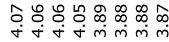

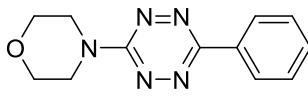
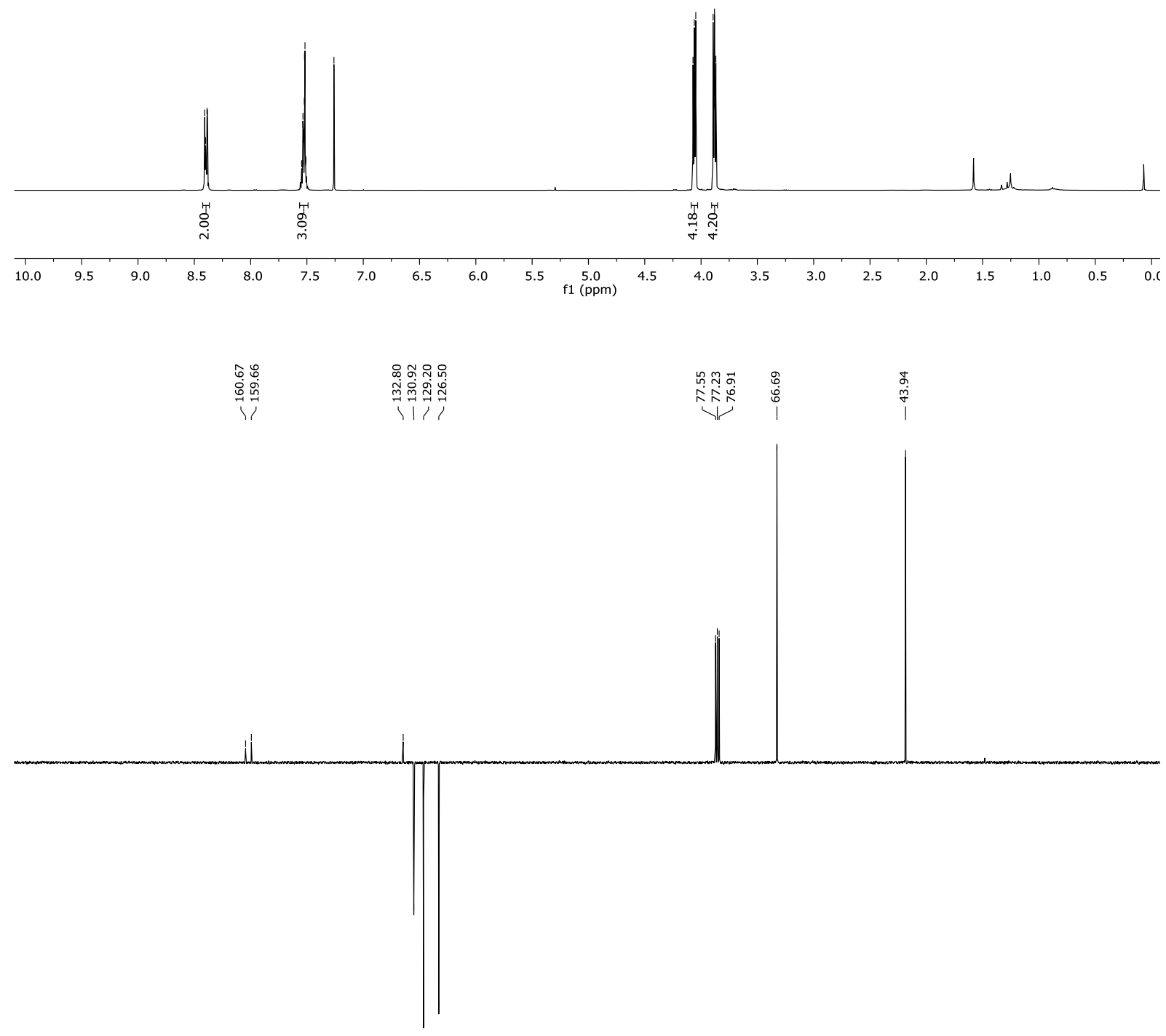

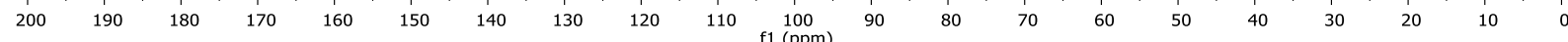


${ }^{1} \mathrm{H}$ and ${ }^{13} \mathrm{C}$ NMR spectra of tetrazine $\mathbf{S 2 0}$
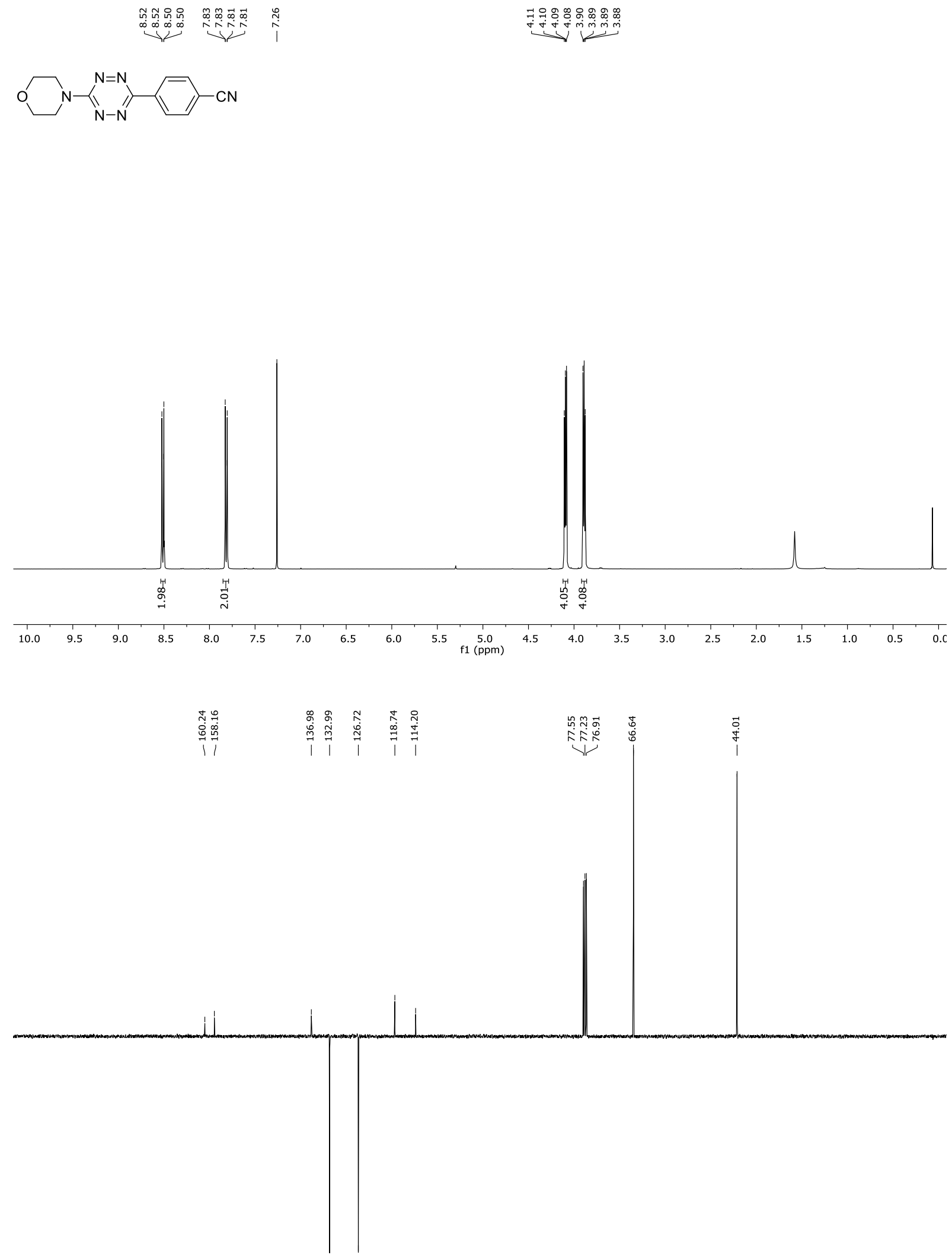

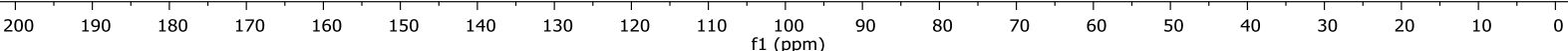


${ }^{1} \mathrm{H}$ and ${ }^{13} \mathrm{C}$ NMR spectra of tetrazine $\mathbf{S 2 2}$
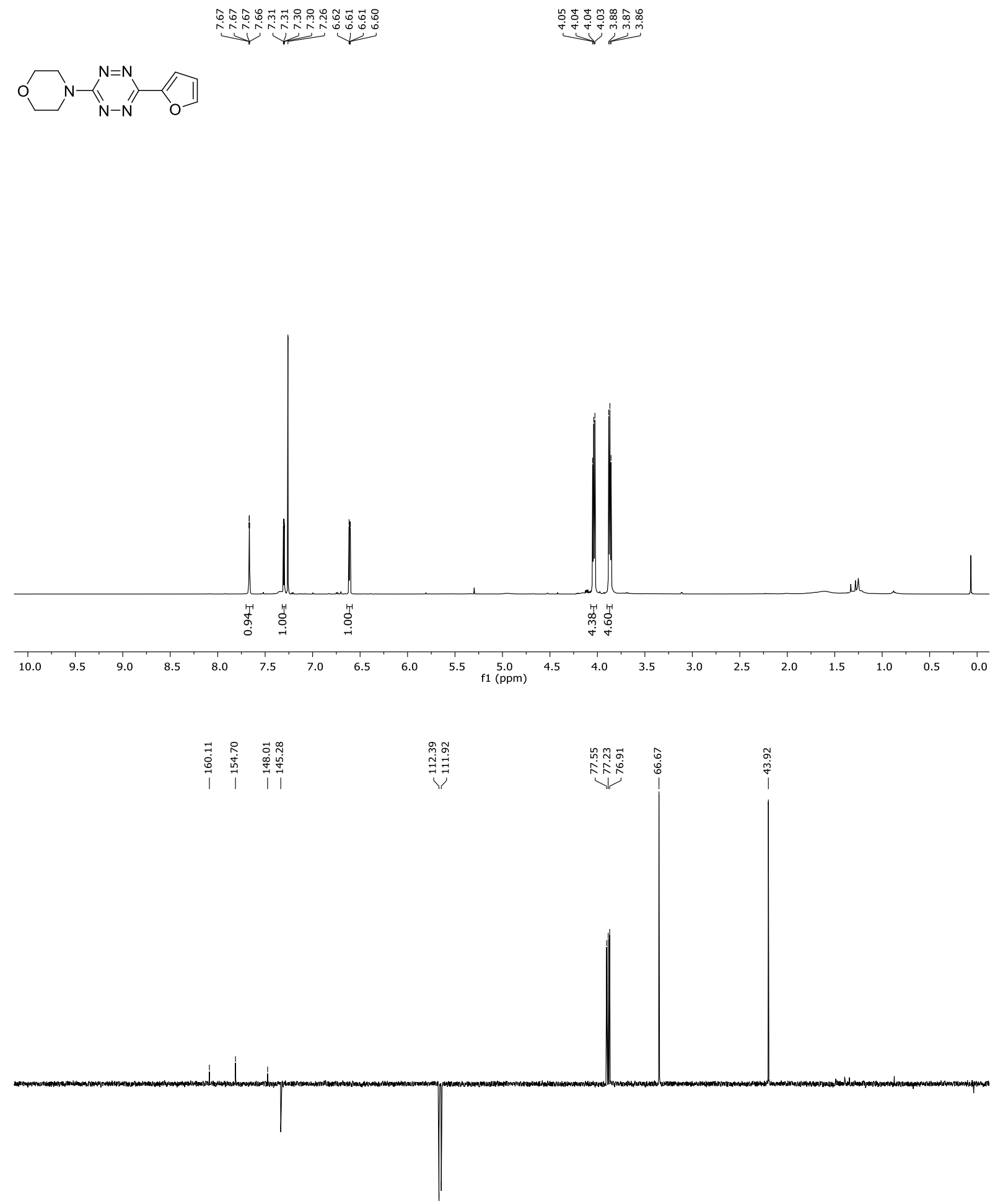

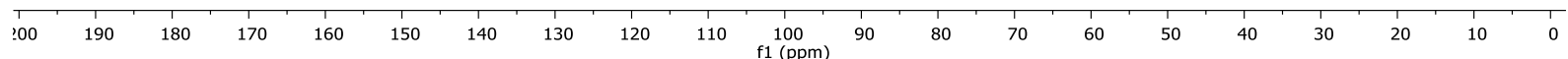


${ }^{1} \mathrm{H}$ and ${ }^{13} \mathrm{C}$ NMR spectra of tetrazine S24

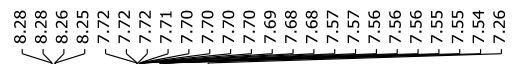

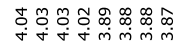

$\overbrace{}^{0}$
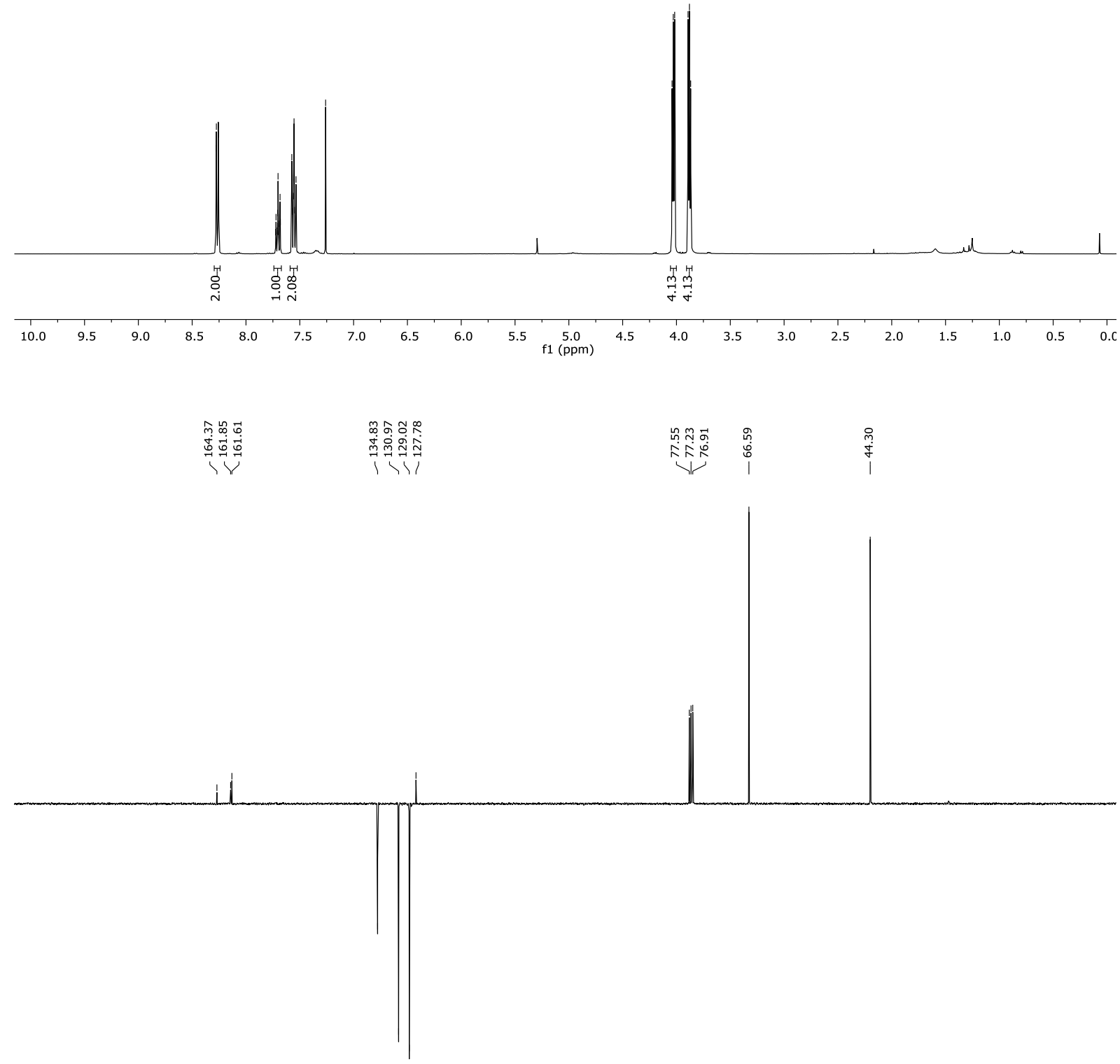

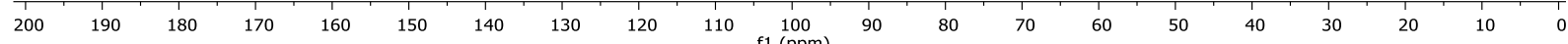


${ }^{1} \mathrm{H}$ and ${ }^{13} \mathrm{C}$ NMR spectra of tetrazine $\mathbf{S 2 6}$
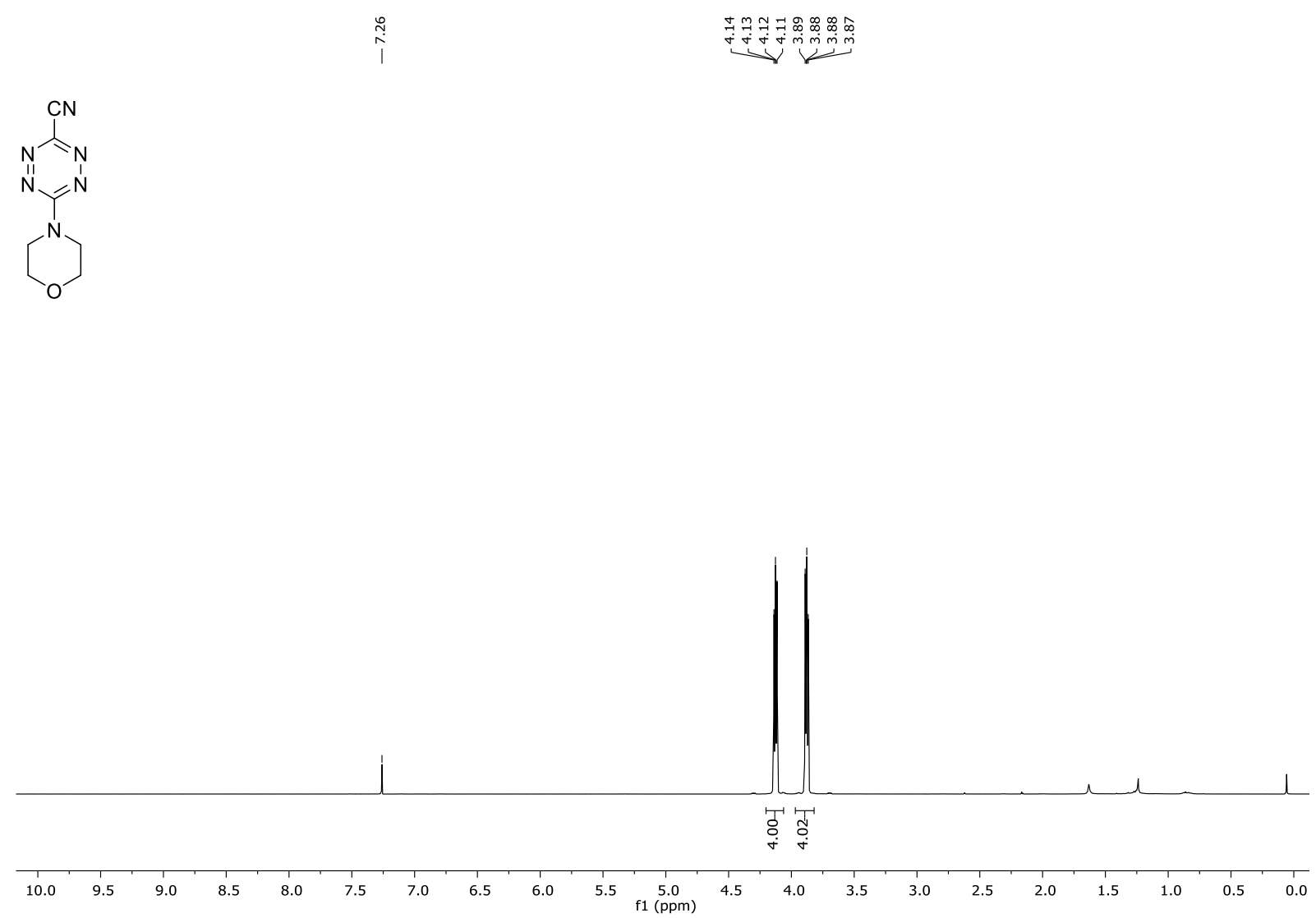

\begin{tabular}{|c|c|c|c|}
\hline 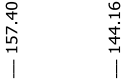 & $\begin{array}{l}\stackrel{+}{a} \\
\stackrel{+}{+} \\
\overrightarrow{1}\end{array}$ & 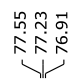 & $\begin{array}{l}q \\
b \\
0 \\
1\end{array}$ \\
\hline
\end{tabular}

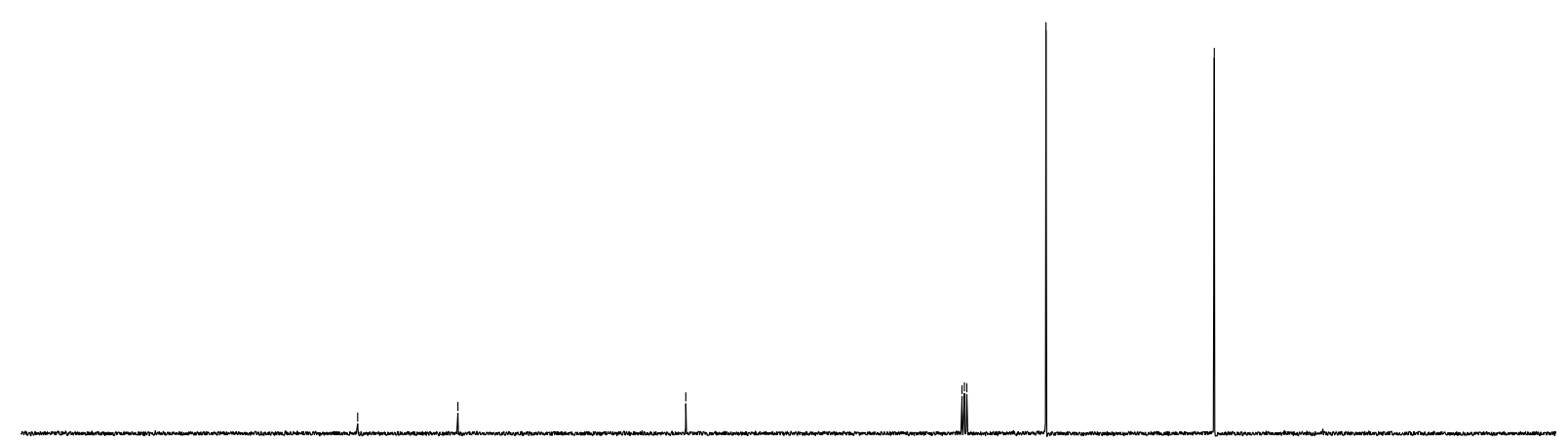

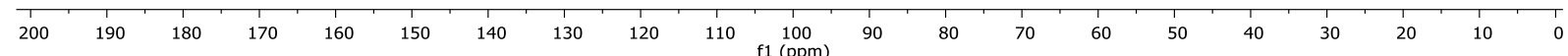


${ }^{1} \mathrm{H}$ and ${ }^{13} \mathrm{C}$ NMR spectra of tetrazine $\mathbf{S 2 7}$

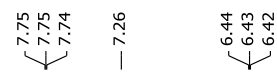

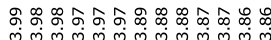

${ }^{0}$

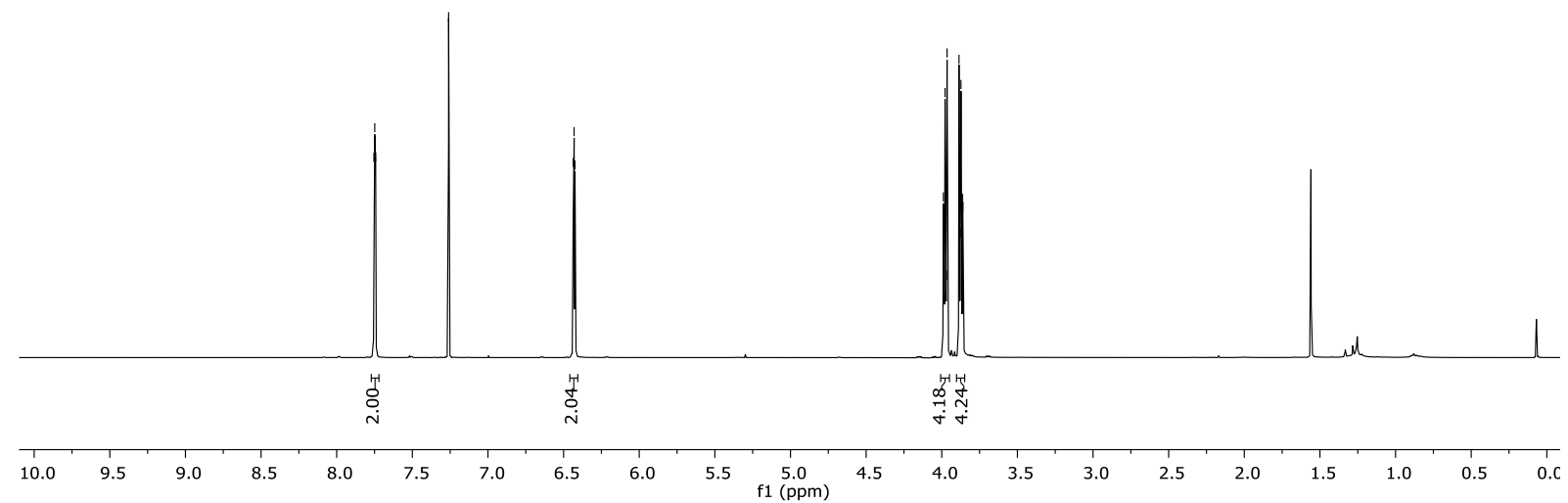

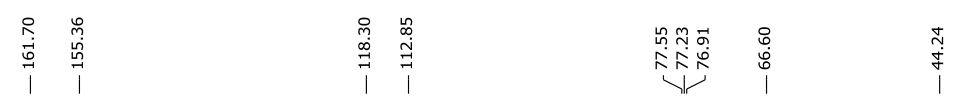

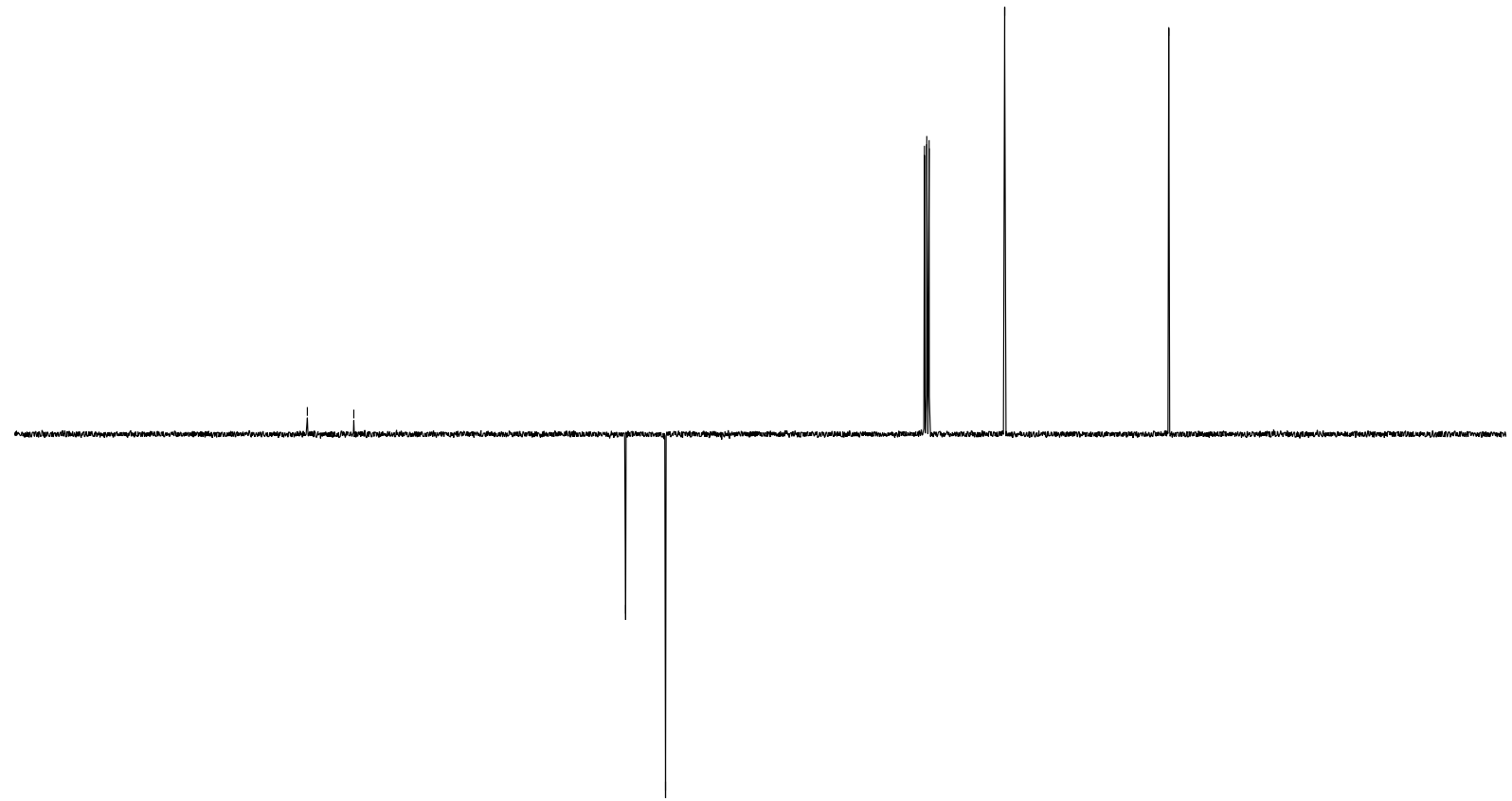

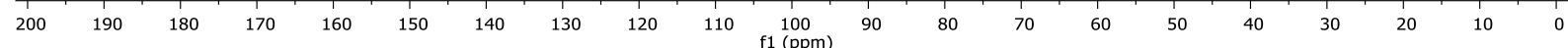




\section{${ }^{1} \mathrm{H}$ and ${ }^{13} \mathrm{C}$ NMR spectra of $\mathbf{6 a}$}

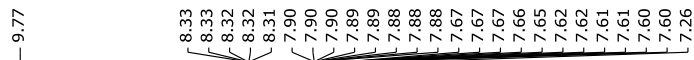
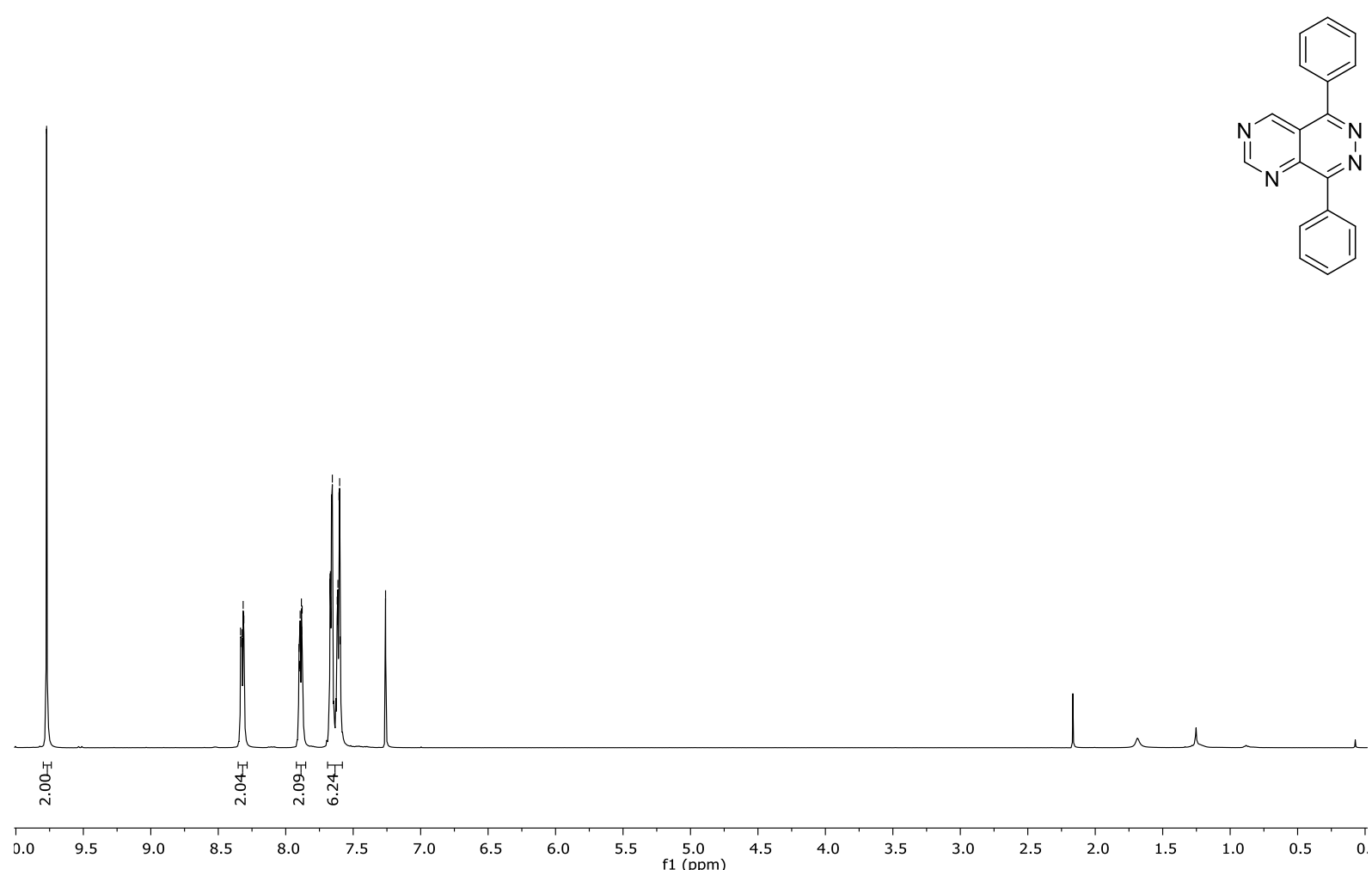

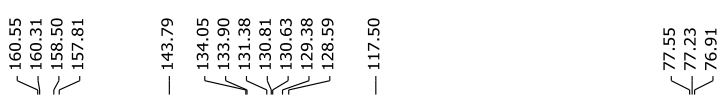

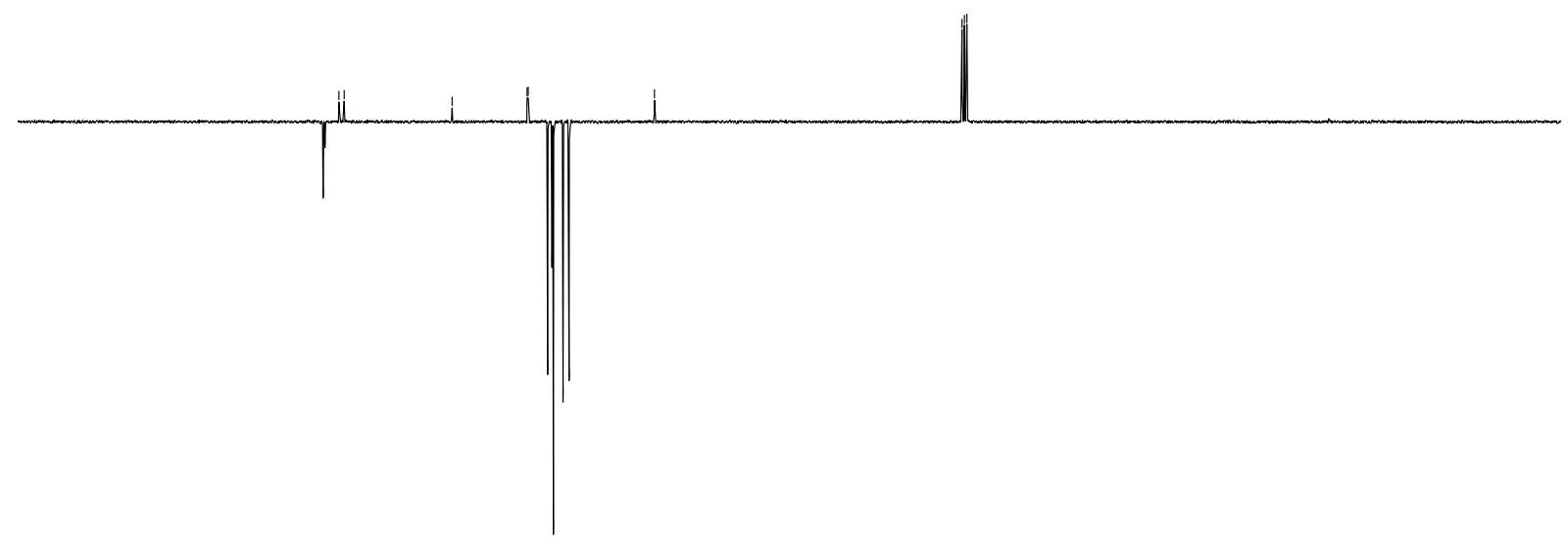

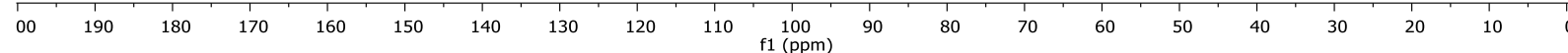




\section{${ }^{1} \mathrm{H}$ and ${ }^{13} \mathrm{C}$ NMR spectra of $\mathbf{6 b}$}

F.
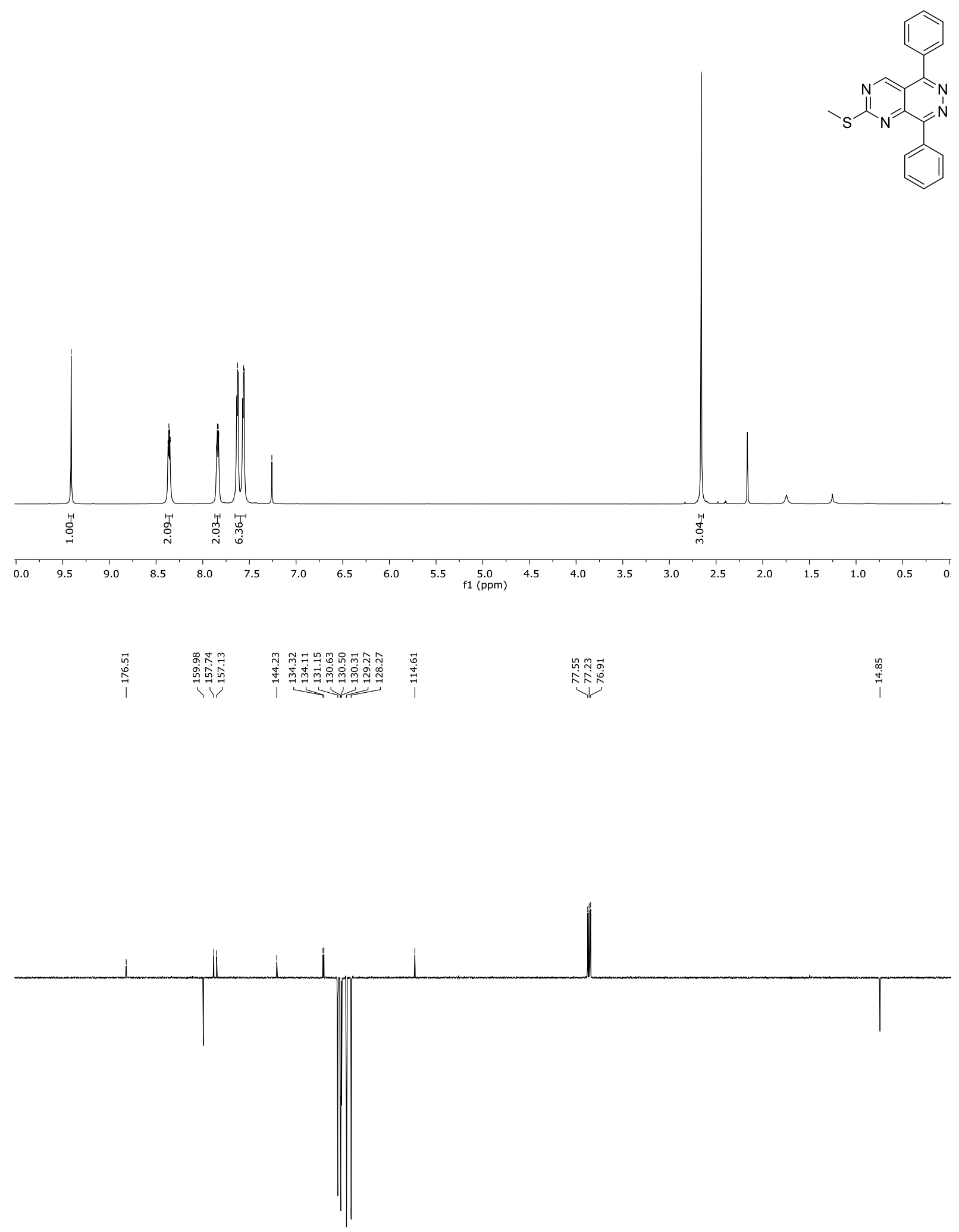

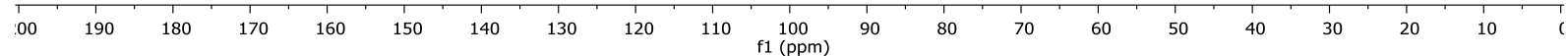


${ }^{1} \mathrm{H}$ and ${ }^{13} \mathrm{C}$ NMR spectra of $6 \mathrm{c}$

$\underbrace{\infty}$

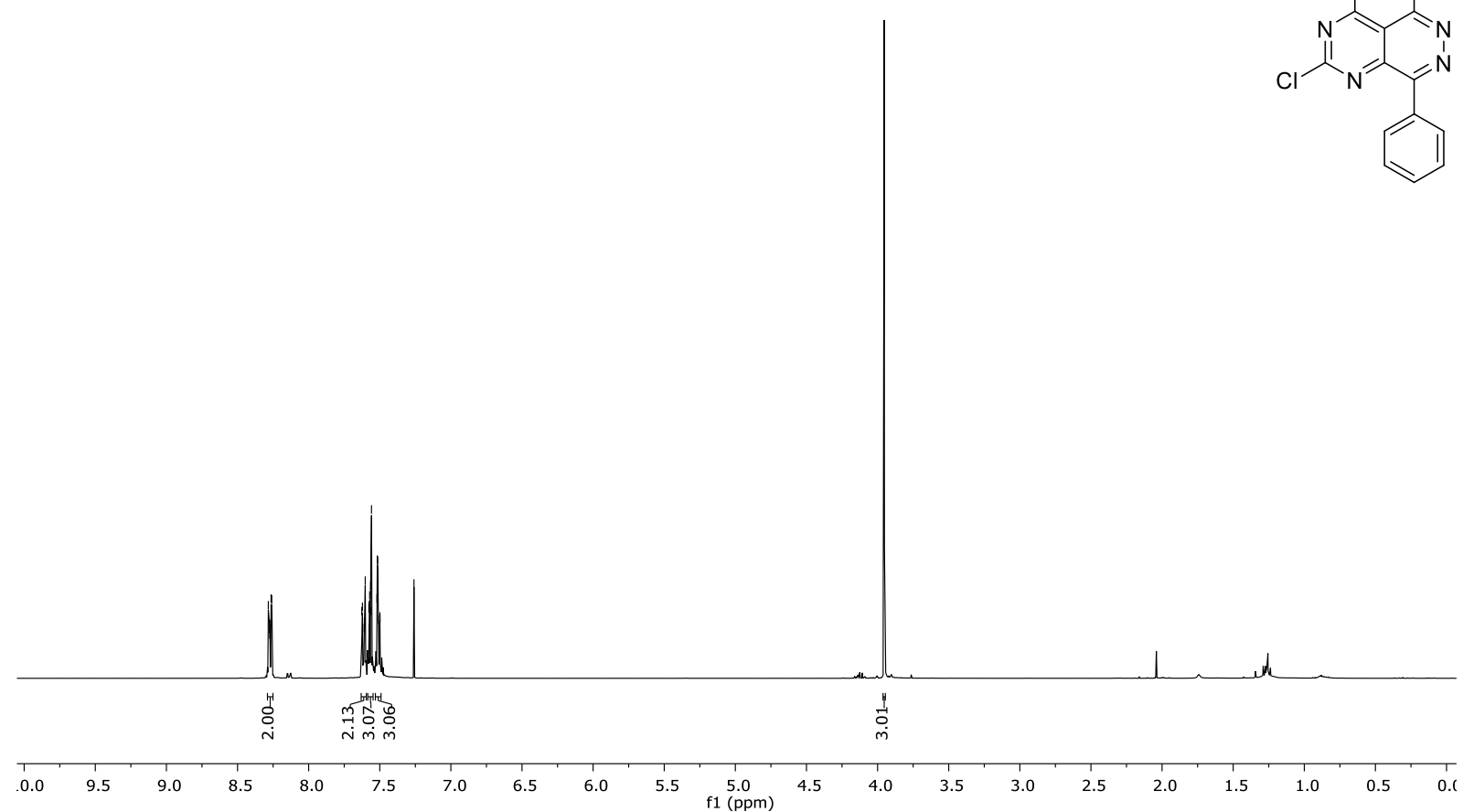

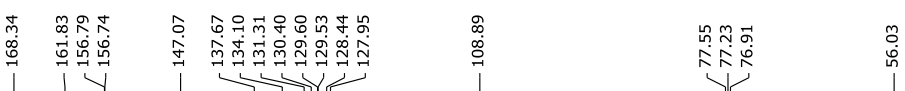

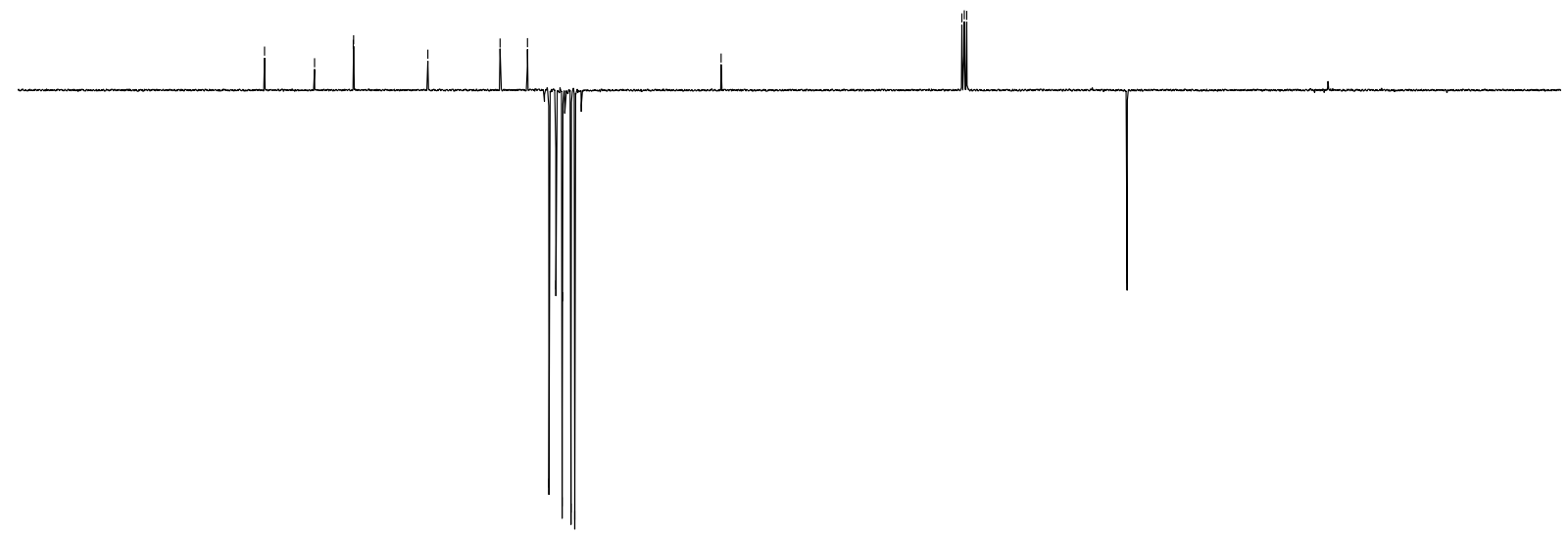

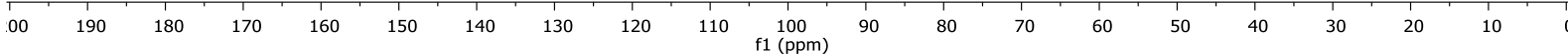


${ }^{1} \mathrm{H}$ and ${ }^{13} \mathrm{C}$ NMR spectra of $6 \mathrm{~d}$ at $298 \mathrm{~K}$

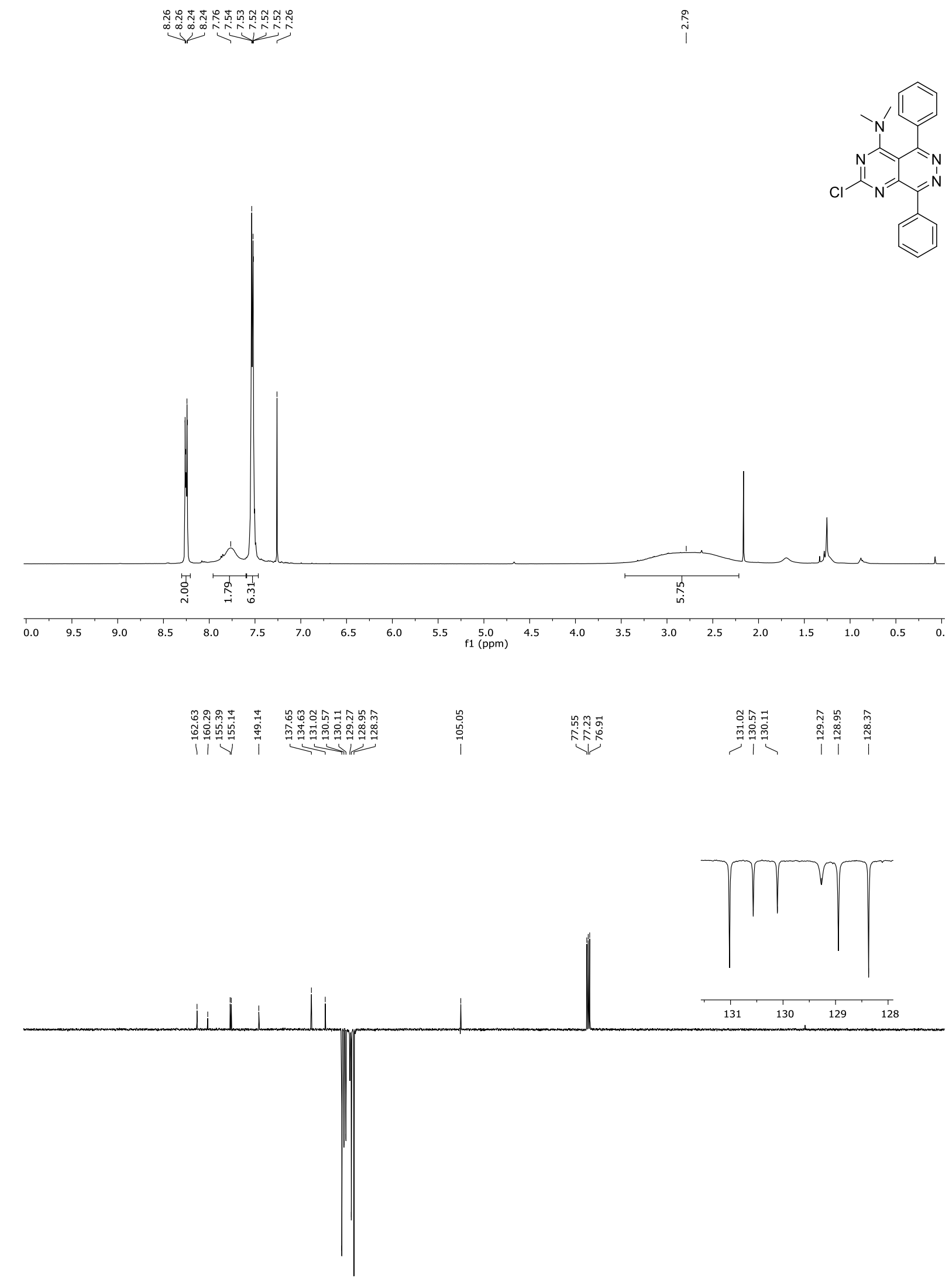

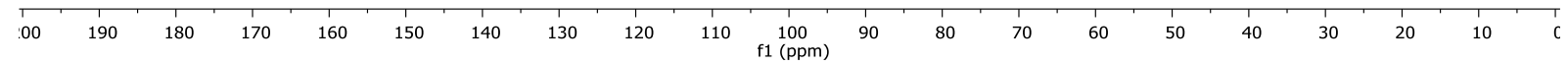


${ }^{1} \mathrm{H}$ and ${ }^{13} \mathrm{C}$ NMR spectra of $6 \mathrm{~d}$ at $238 \mathrm{~K}$

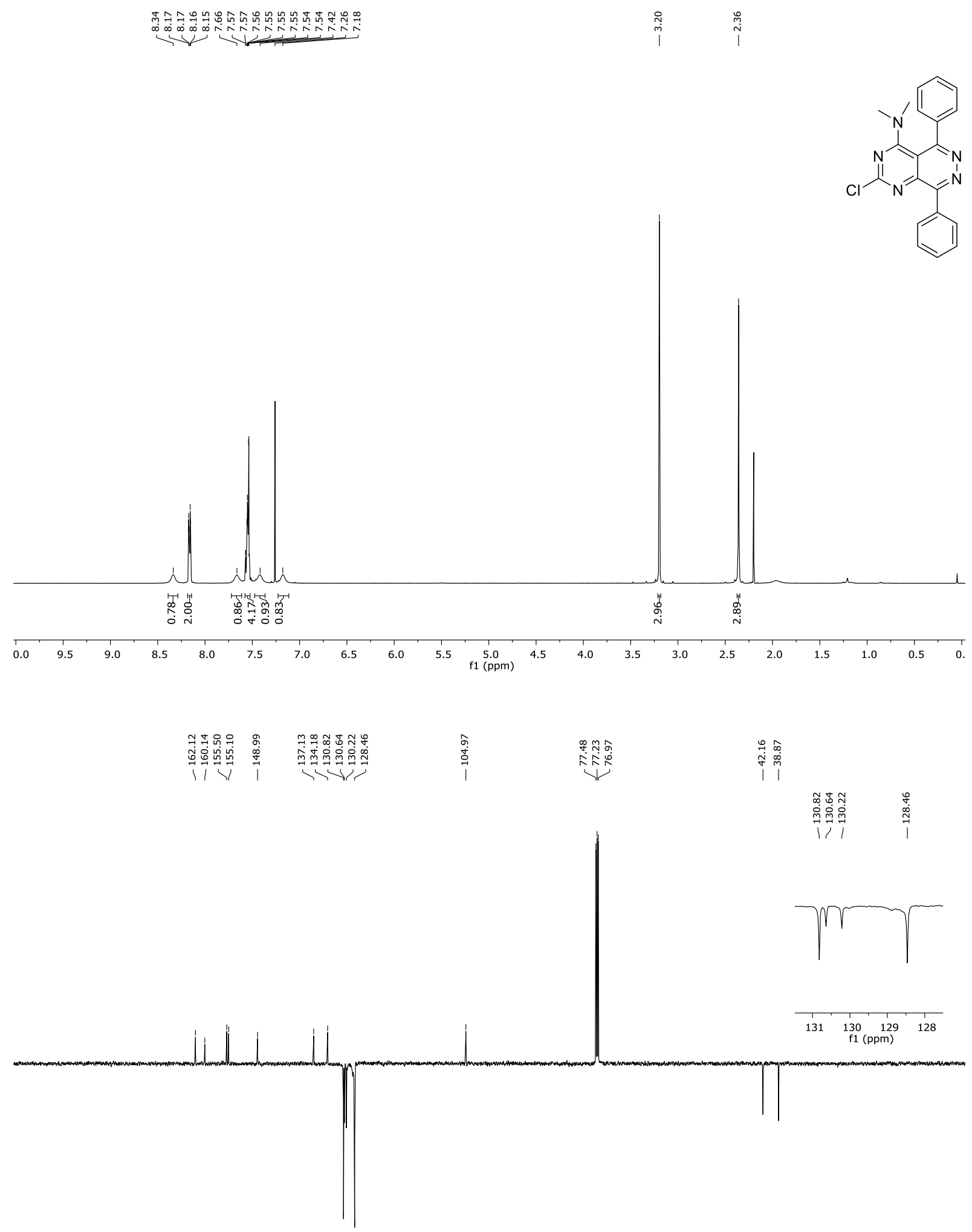

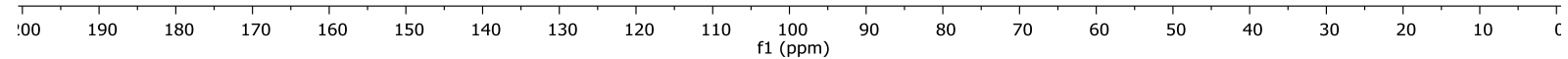


${ }^{1} \mathrm{H}$ and ${ }^{13} \mathrm{C}$ NMR spectra of $6 \mathbf{e}$

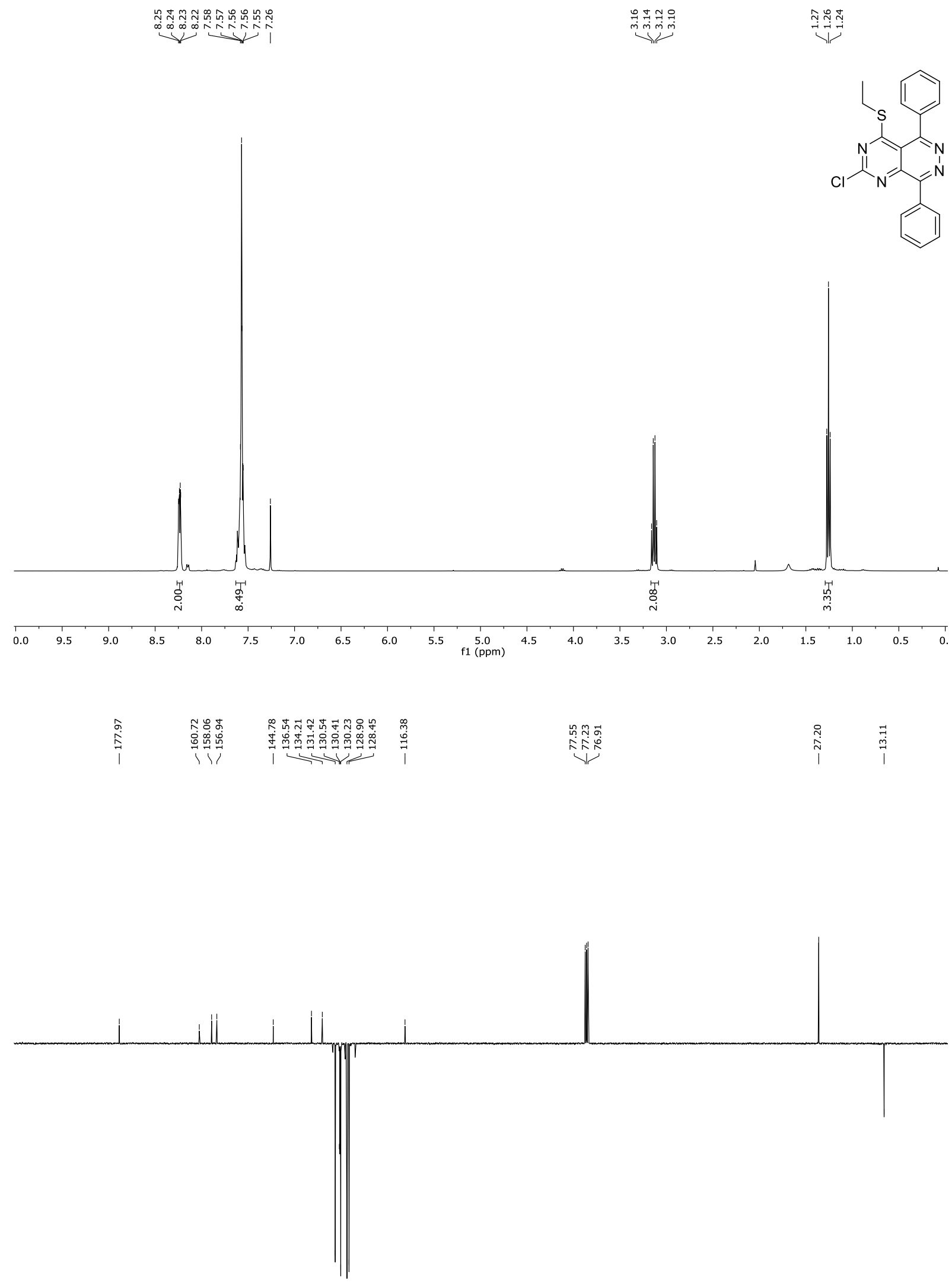

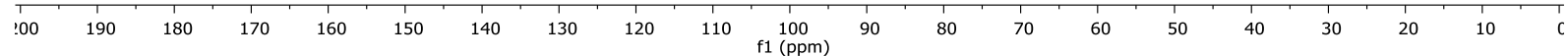


${ }^{1} \mathrm{H}$ and ${ }^{13} \mathrm{C}$ NMR spectra of $6 \mathbf{f}$

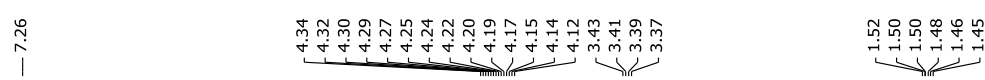
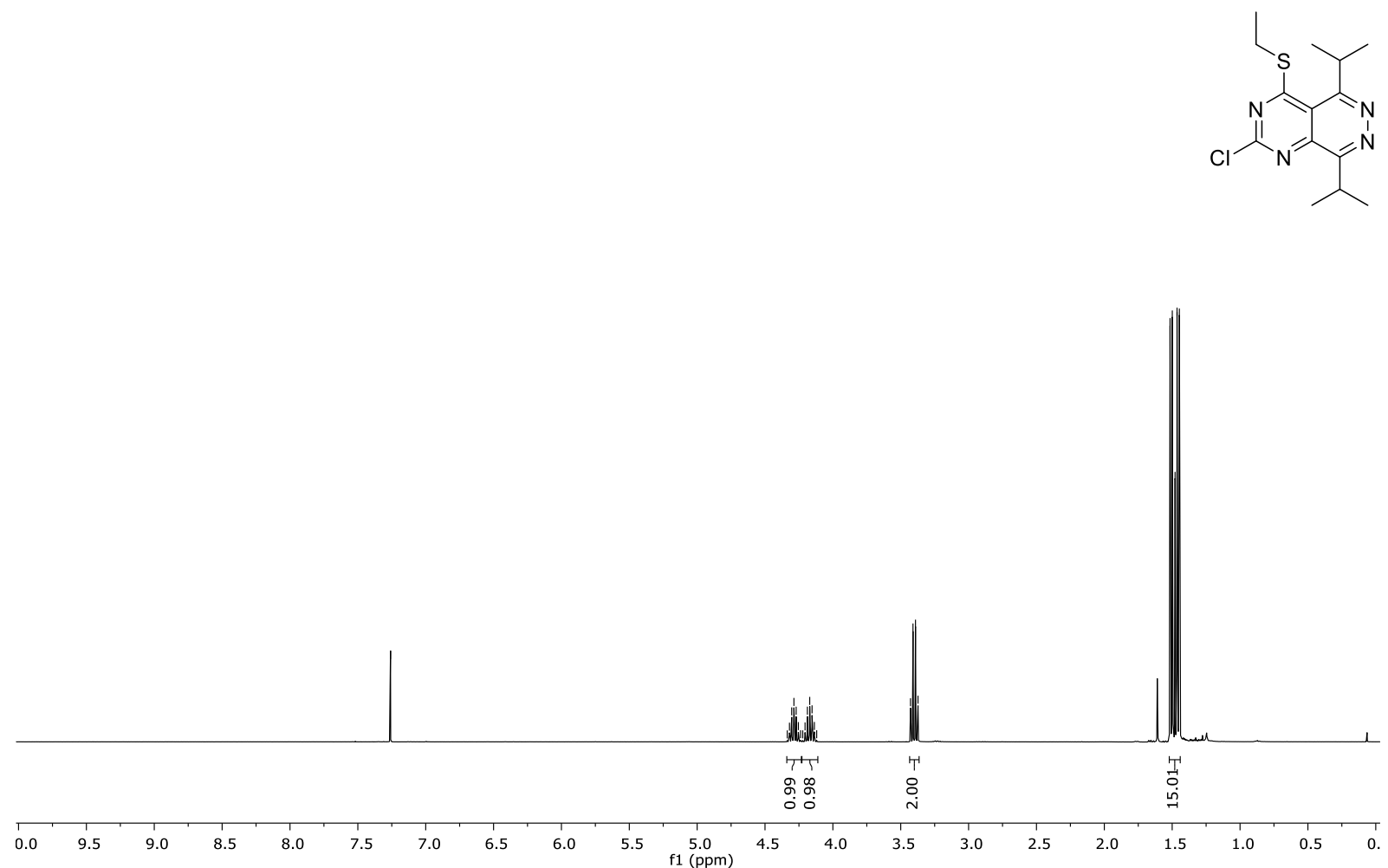

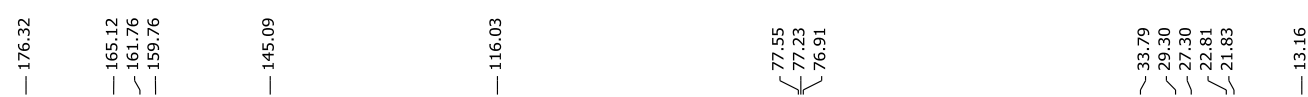

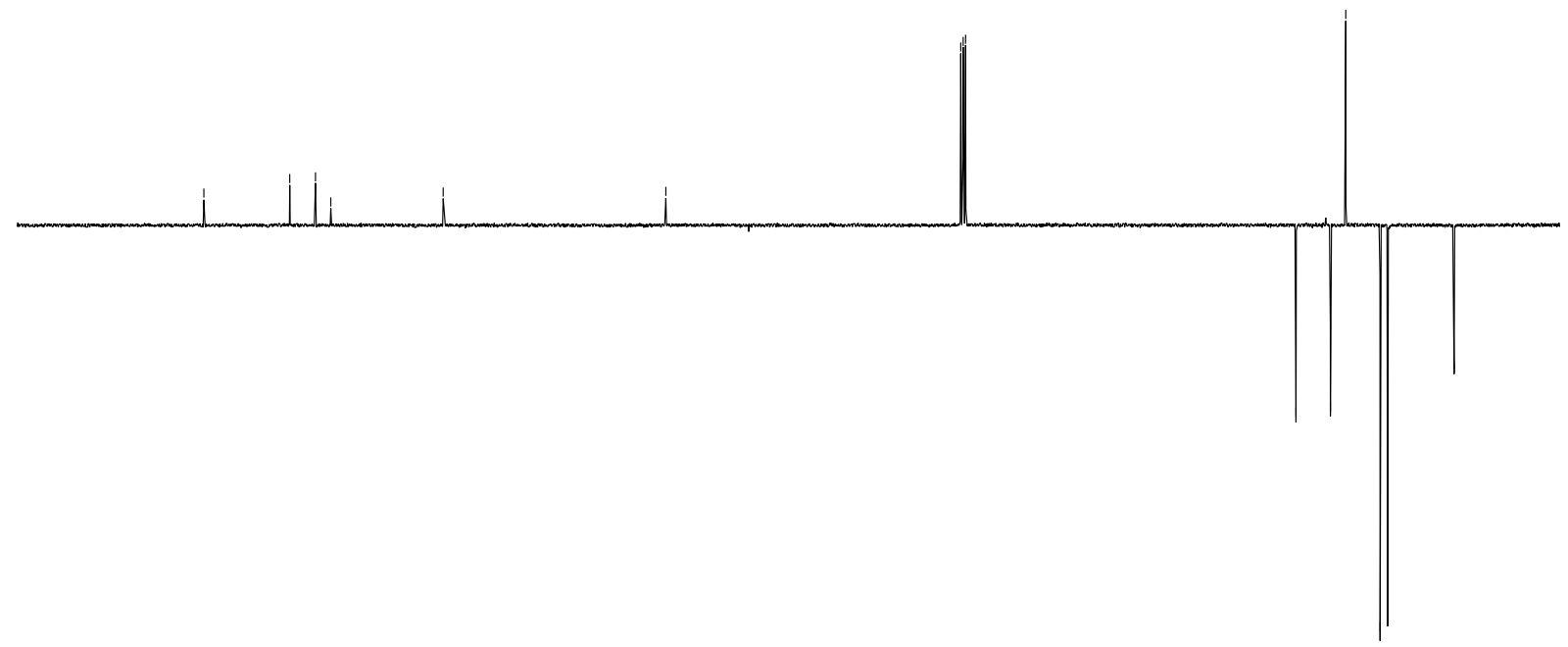

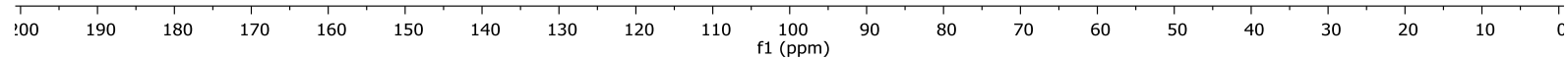




\section{${ }^{1} \mathrm{H}$ and ${ }^{13} \mathrm{C}$ NMR spectra of $6 \mathrm{~g}$}

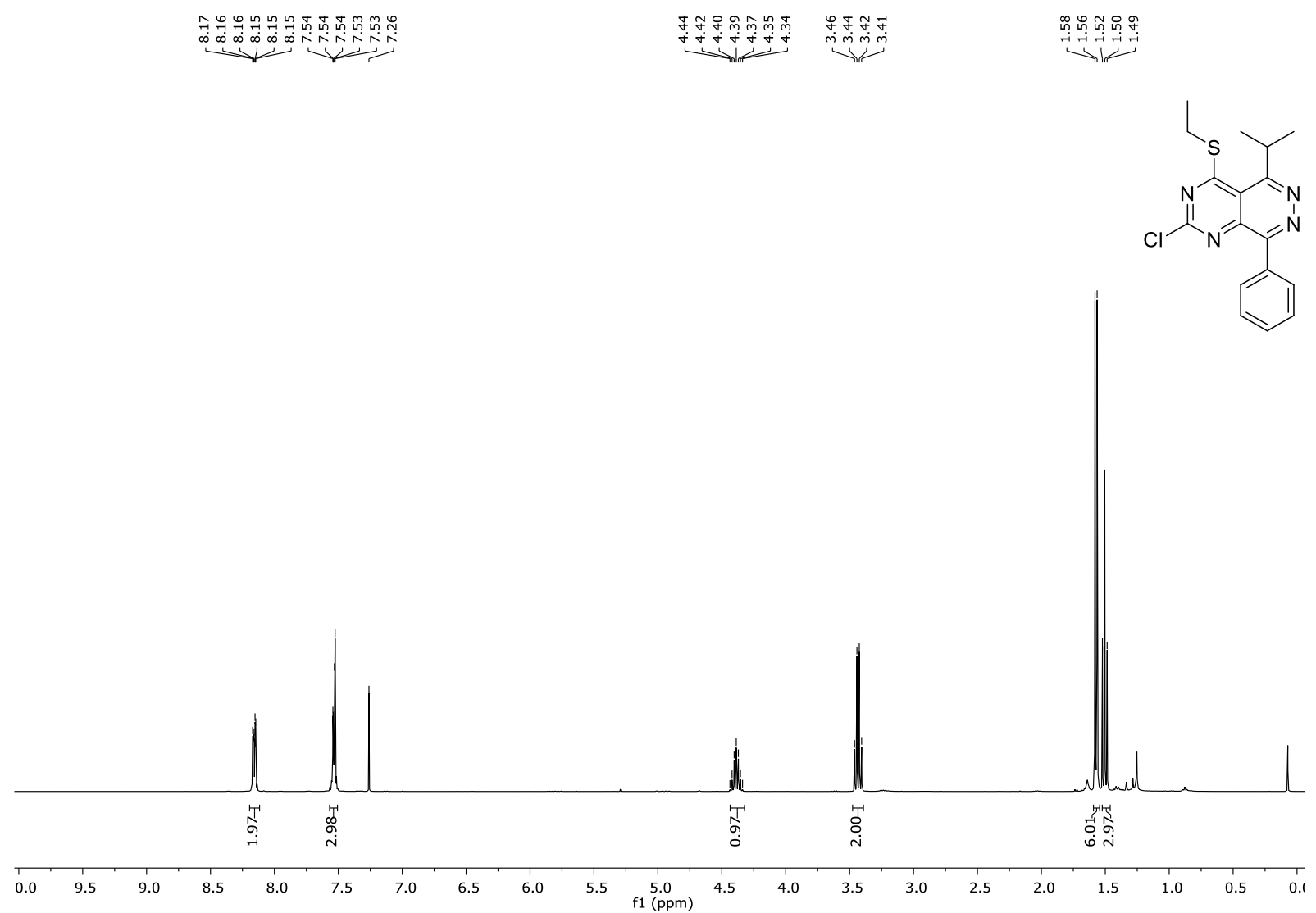

\begin{tabular}{|c|c|c|c|c|}
\hline 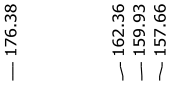 & $\begin{array}{c}\hat{N} \\
\dot{j} \\
\stackrel{j}{\mid} \\
\mid\end{array}$ & 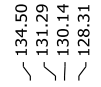 & $\begin{array}{l}\stackrel{0}{\infty} \\
\stackrel{0}{0} \\
\stackrel{1}{1} \\
1\end{array}$ & 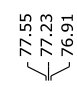 \\
\hline
\end{tabular}

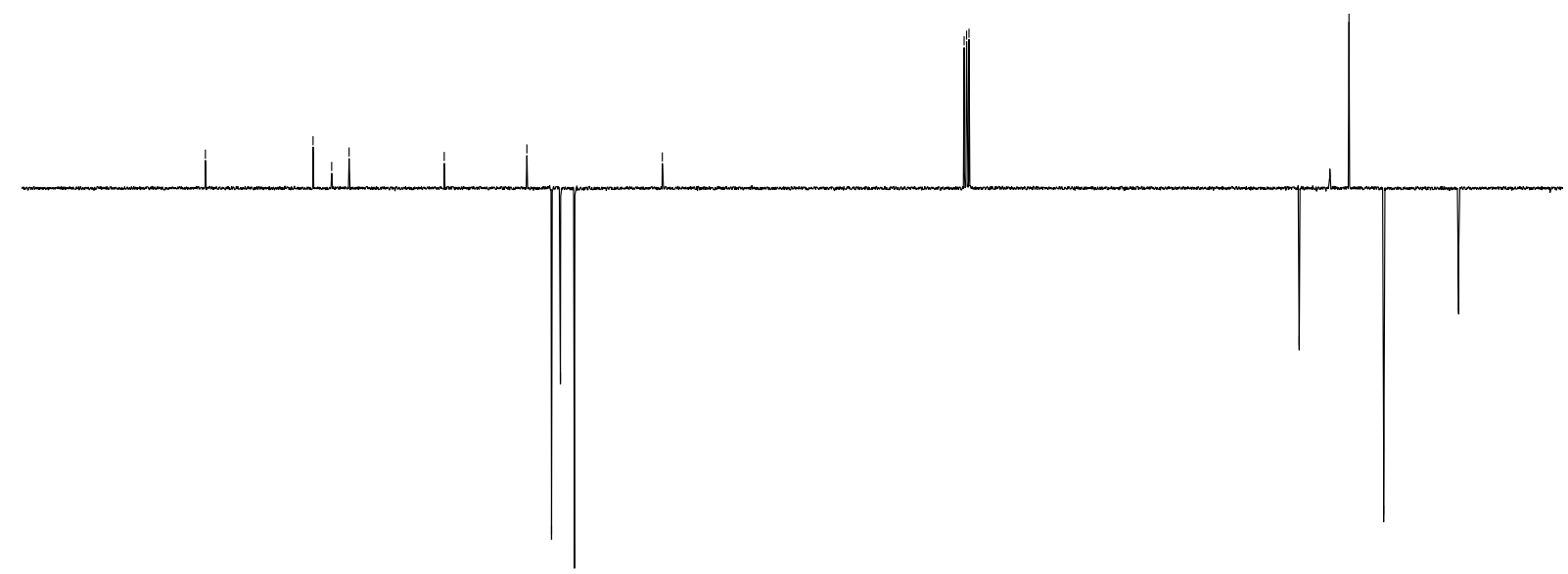

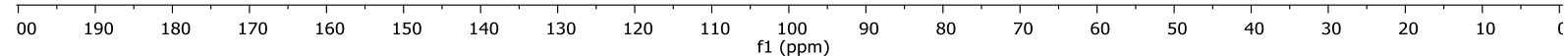


${ }^{1} \mathrm{H}$ and ${ }^{13} \mathrm{C}$ NMR spectra of $6 \mathbf{h}$

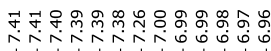

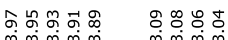

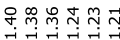

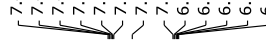

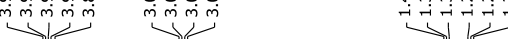

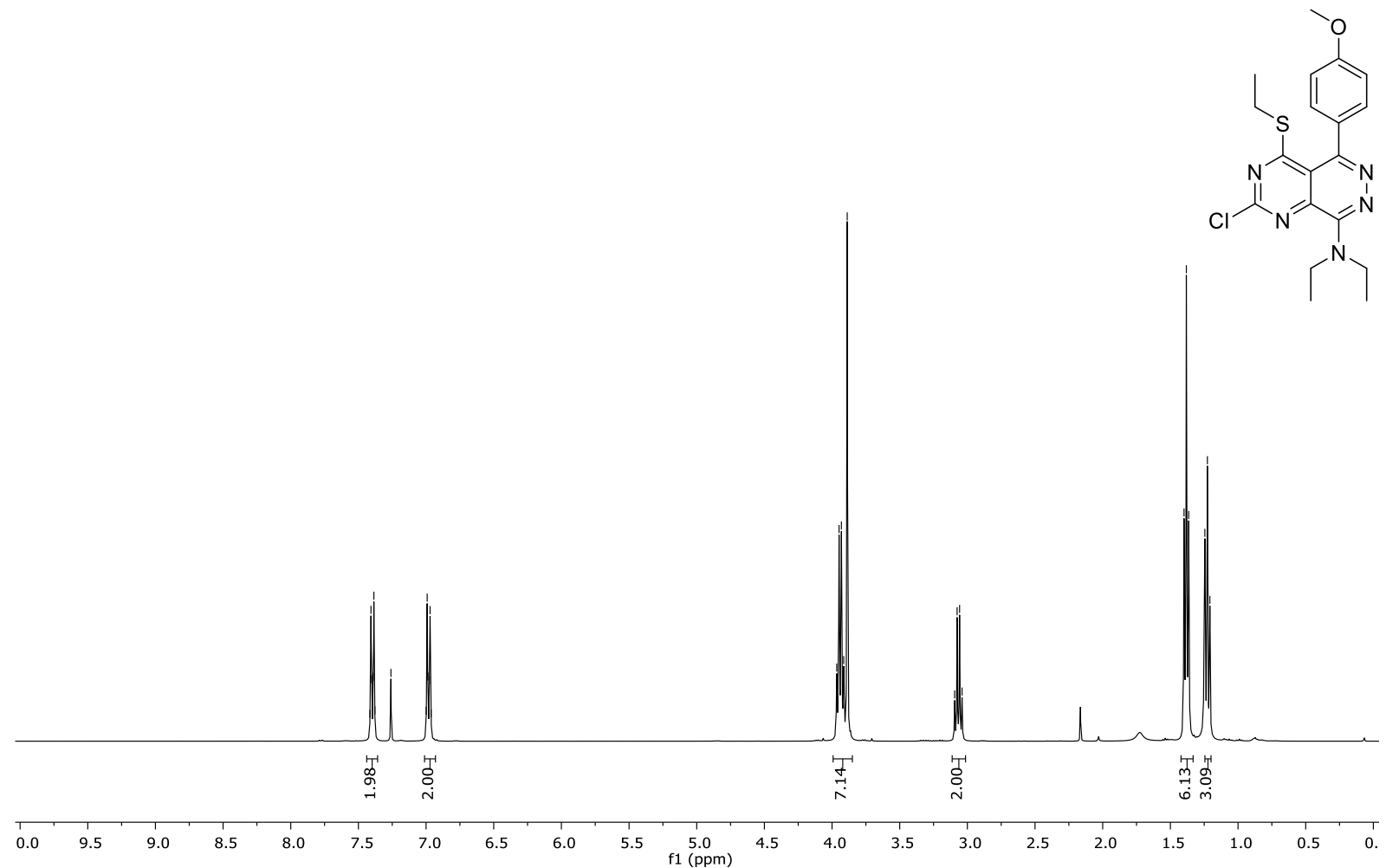

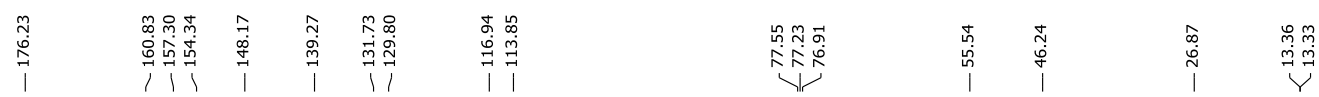

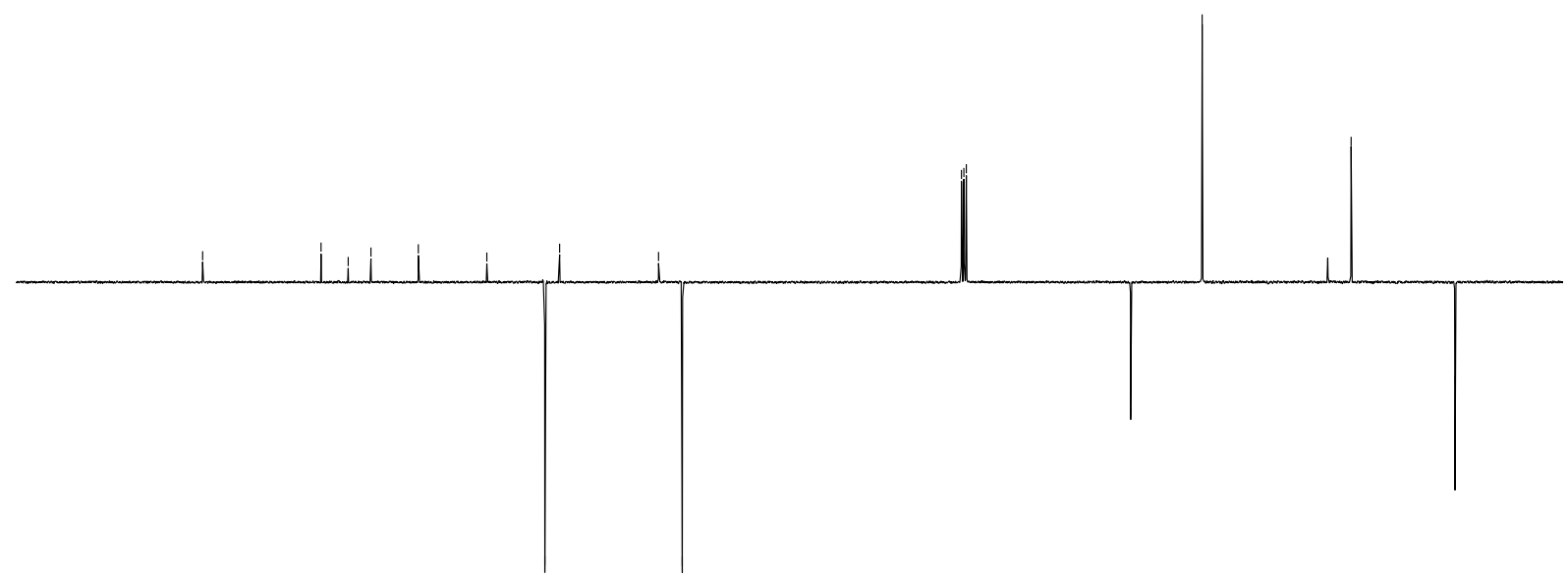

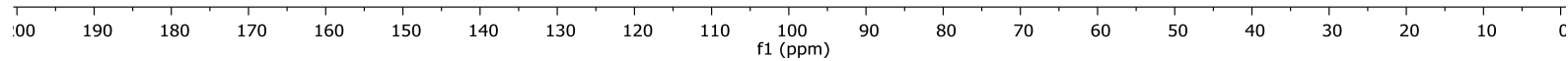


${ }^{1} \mathrm{H}$ and ${ }^{13} \mathrm{C}$ NMR spectra of $\mathbf{6 i}$

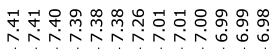

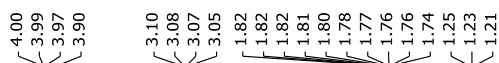

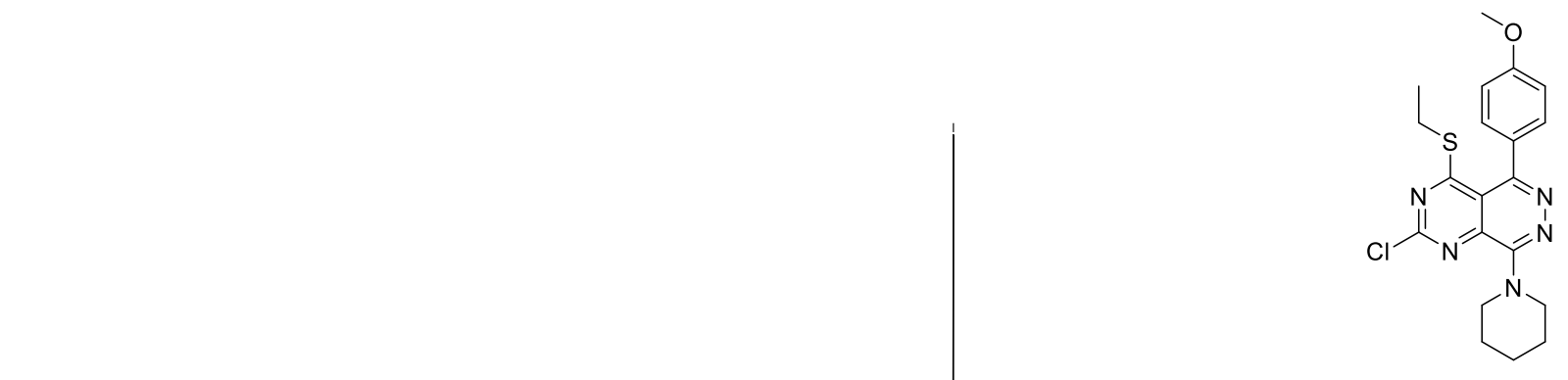

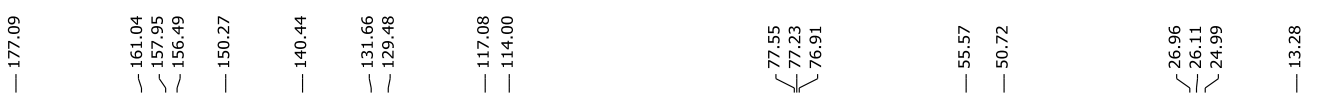

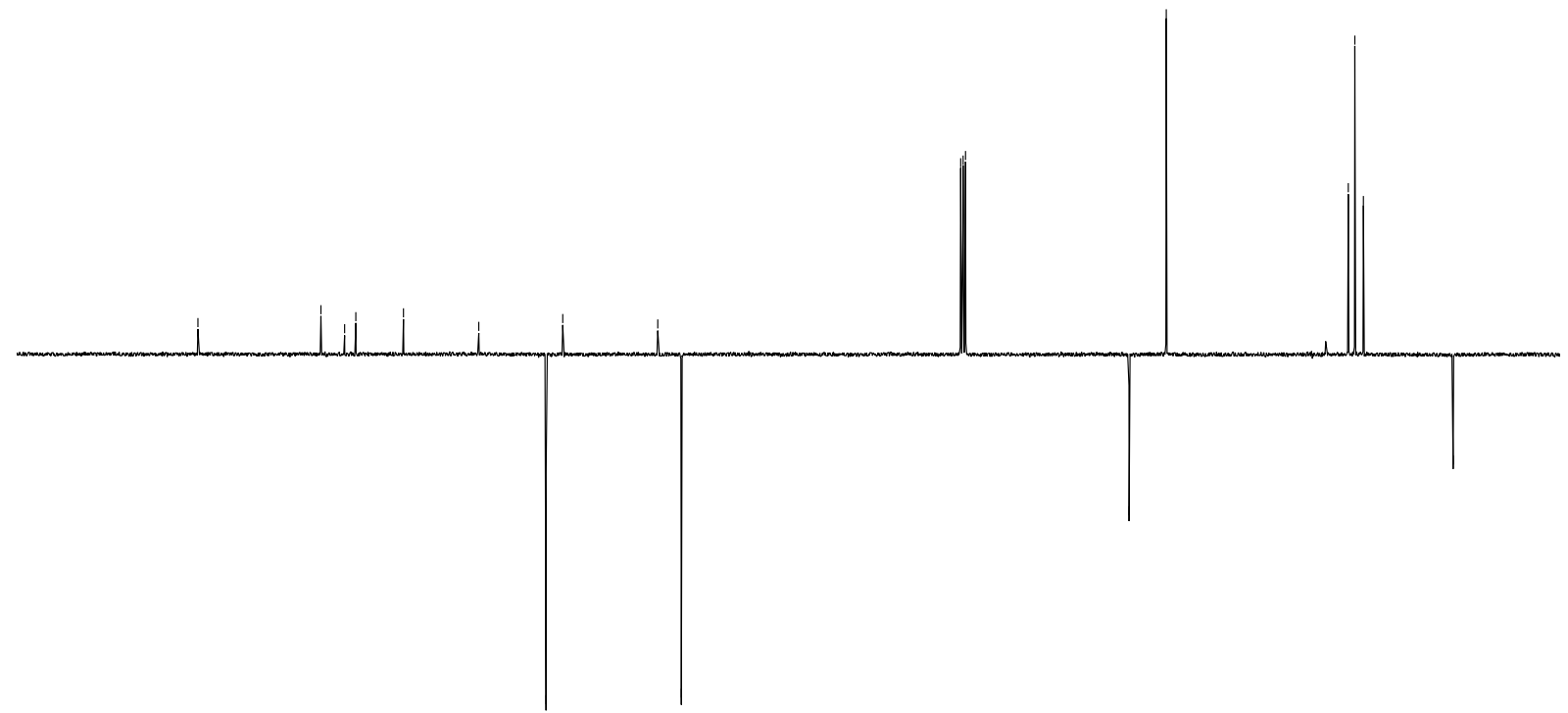

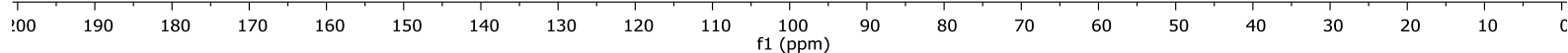




\section{${ }^{1} \mathrm{H}$ and ${ }^{13} \mathrm{C}$ NMR spectra of $\mathbf{6 j}$}

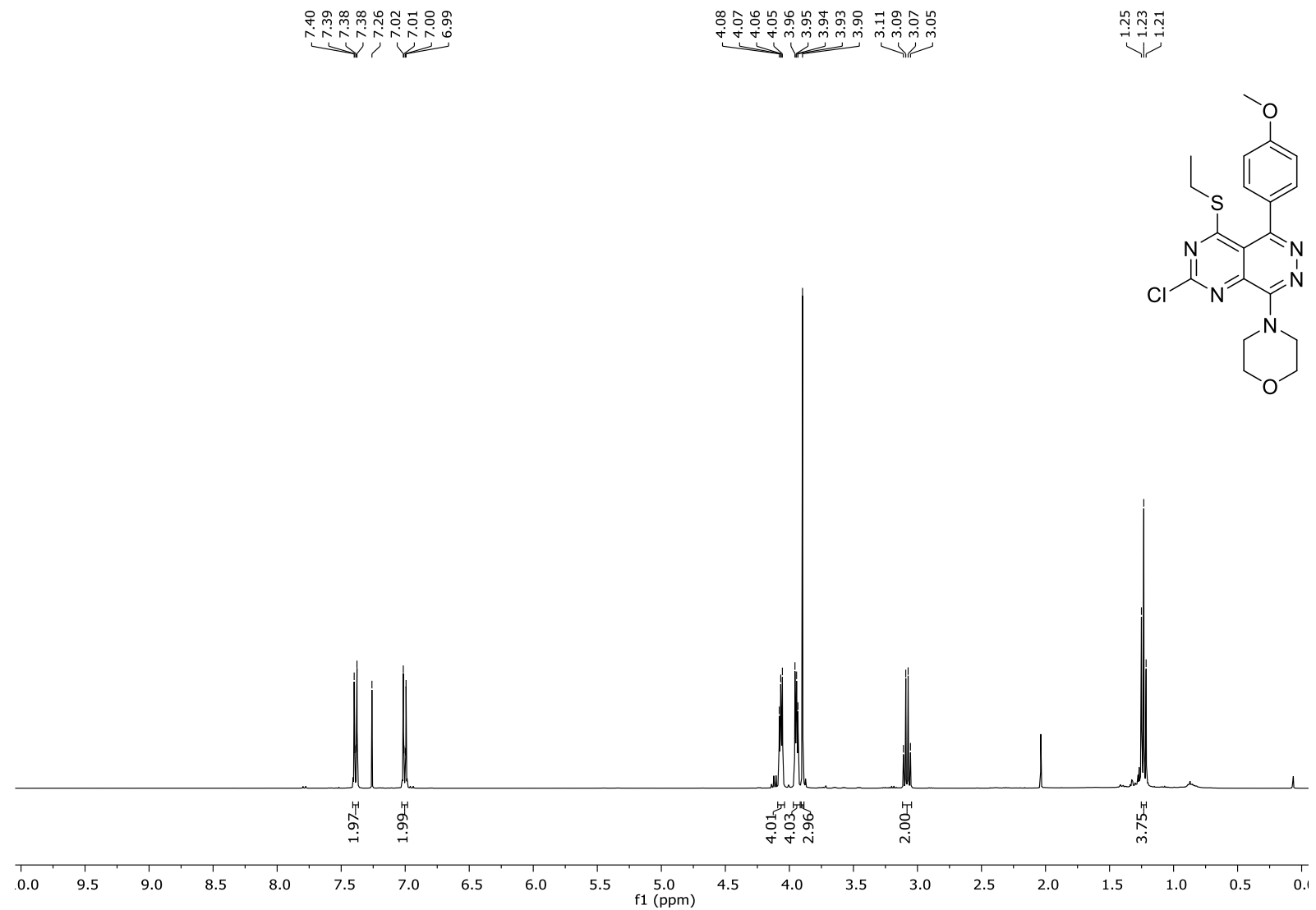

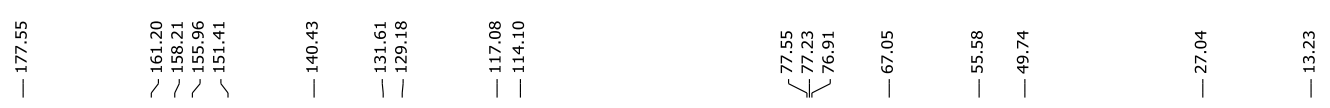

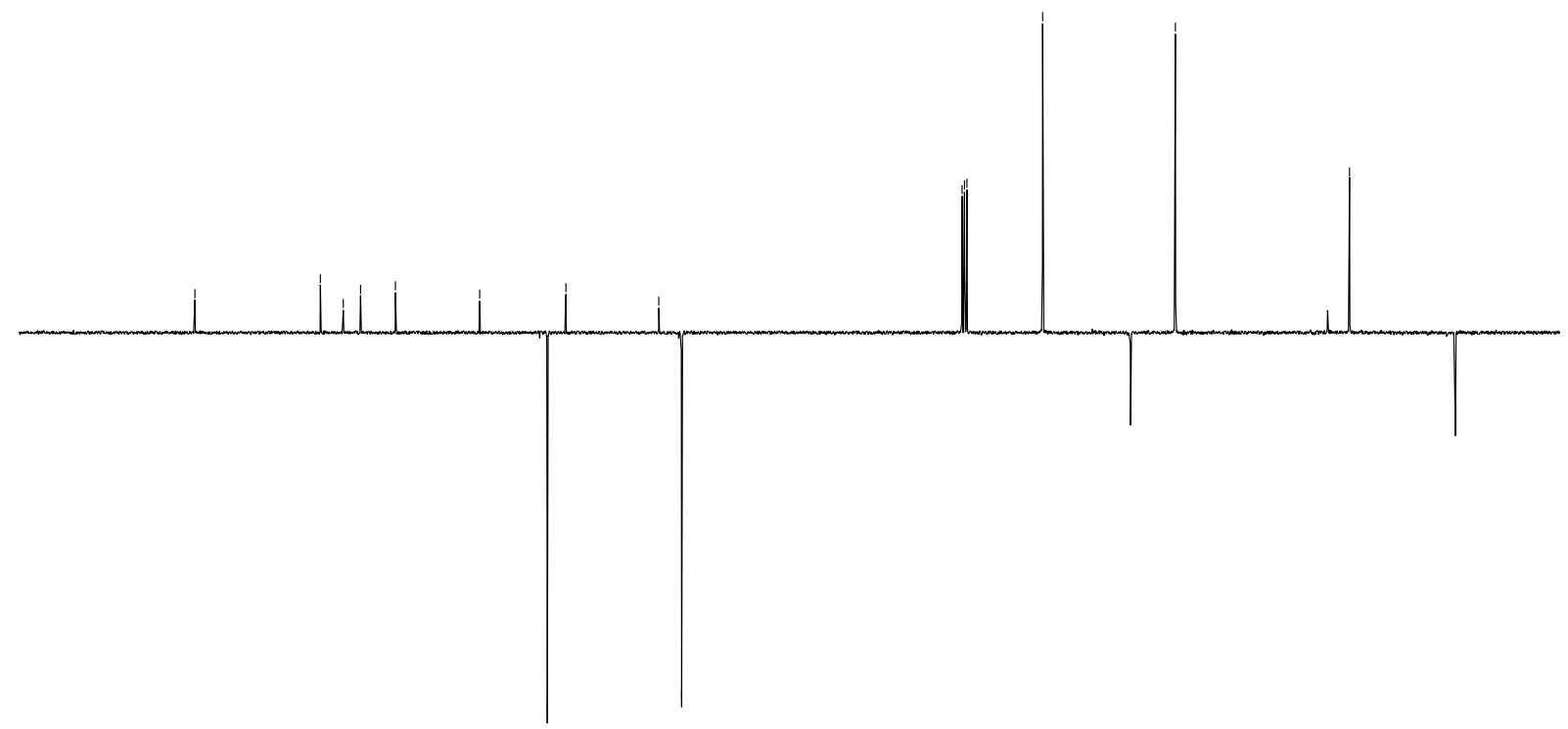

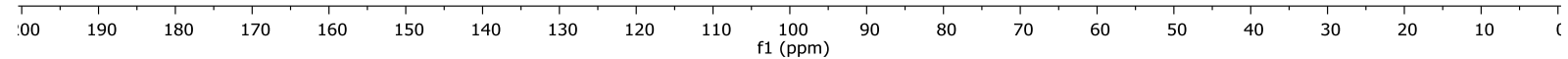


${ }^{1} \mathrm{H}$ and ${ }^{13} \mathrm{C}$ NMR spectra of $\mathbf{6 k}$

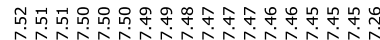

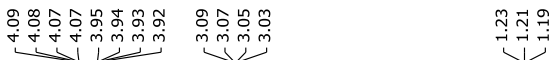

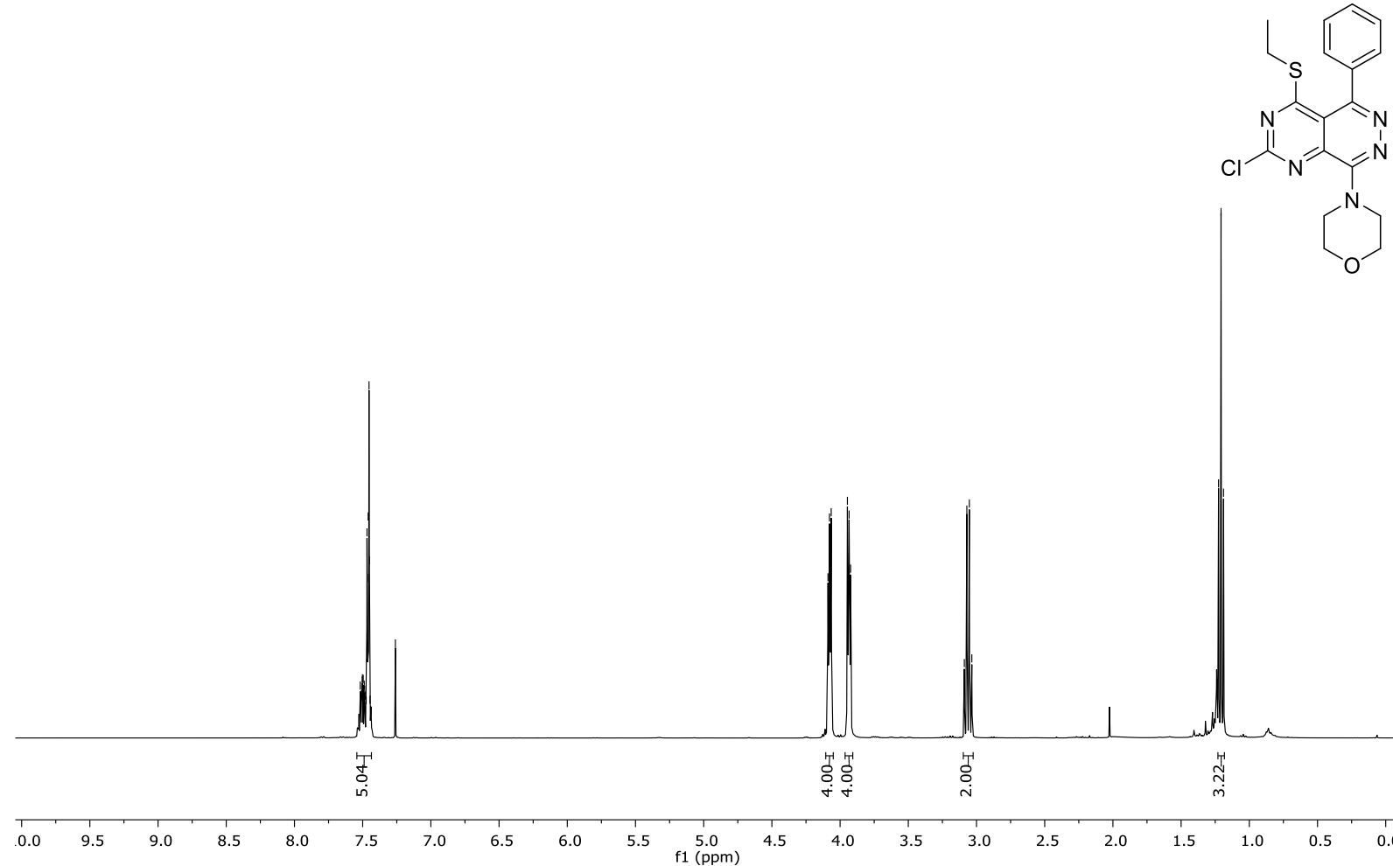

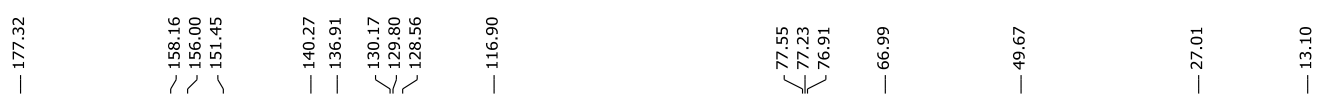

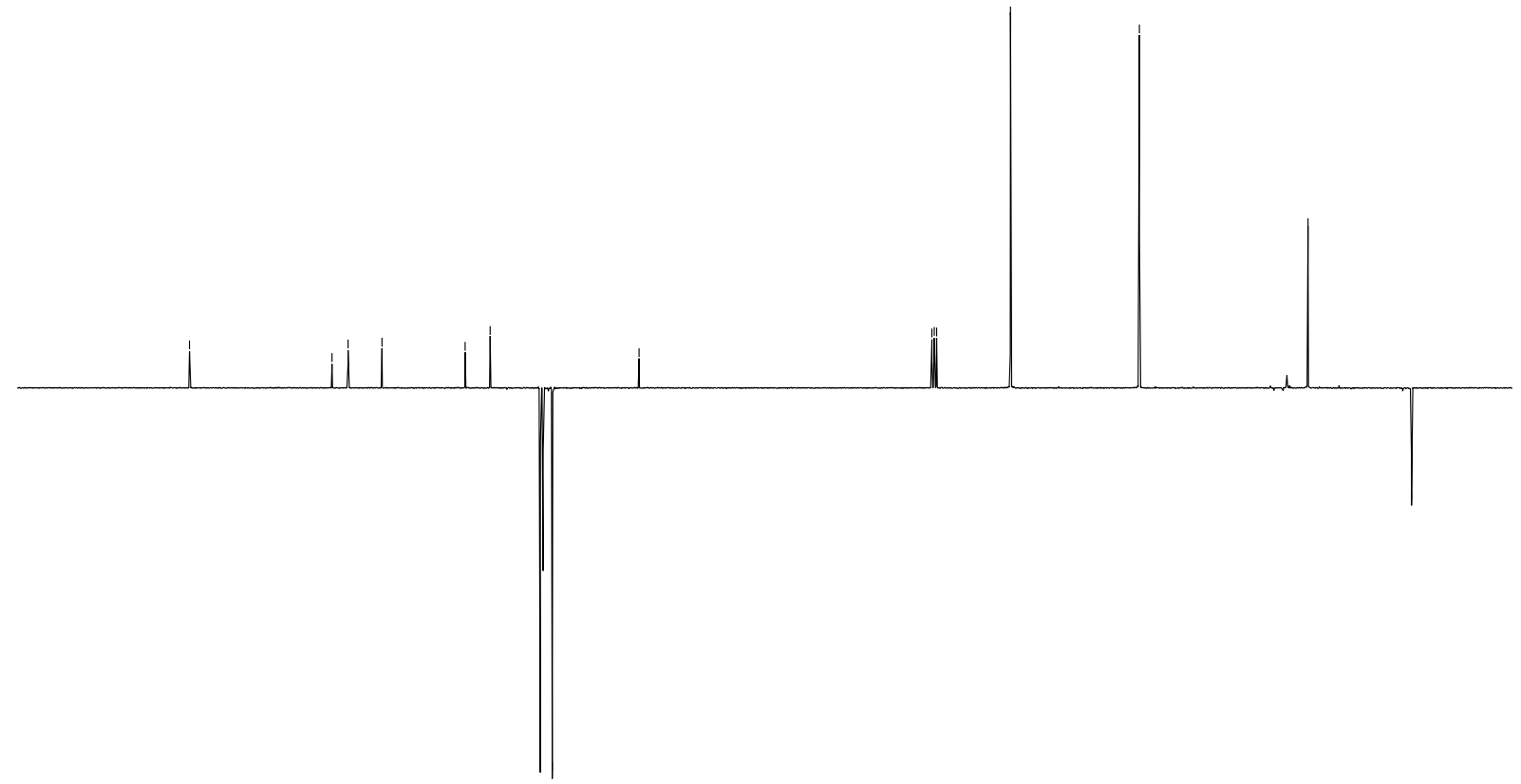

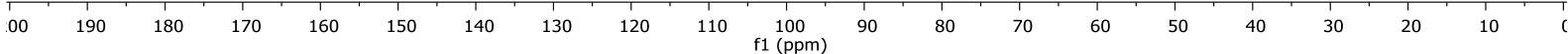


${ }^{1} \mathrm{H}$ and ${ }^{13} \mathrm{C}$ NMR spectra of $6 \mathrm{I}$

年

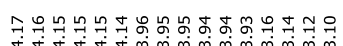

บ

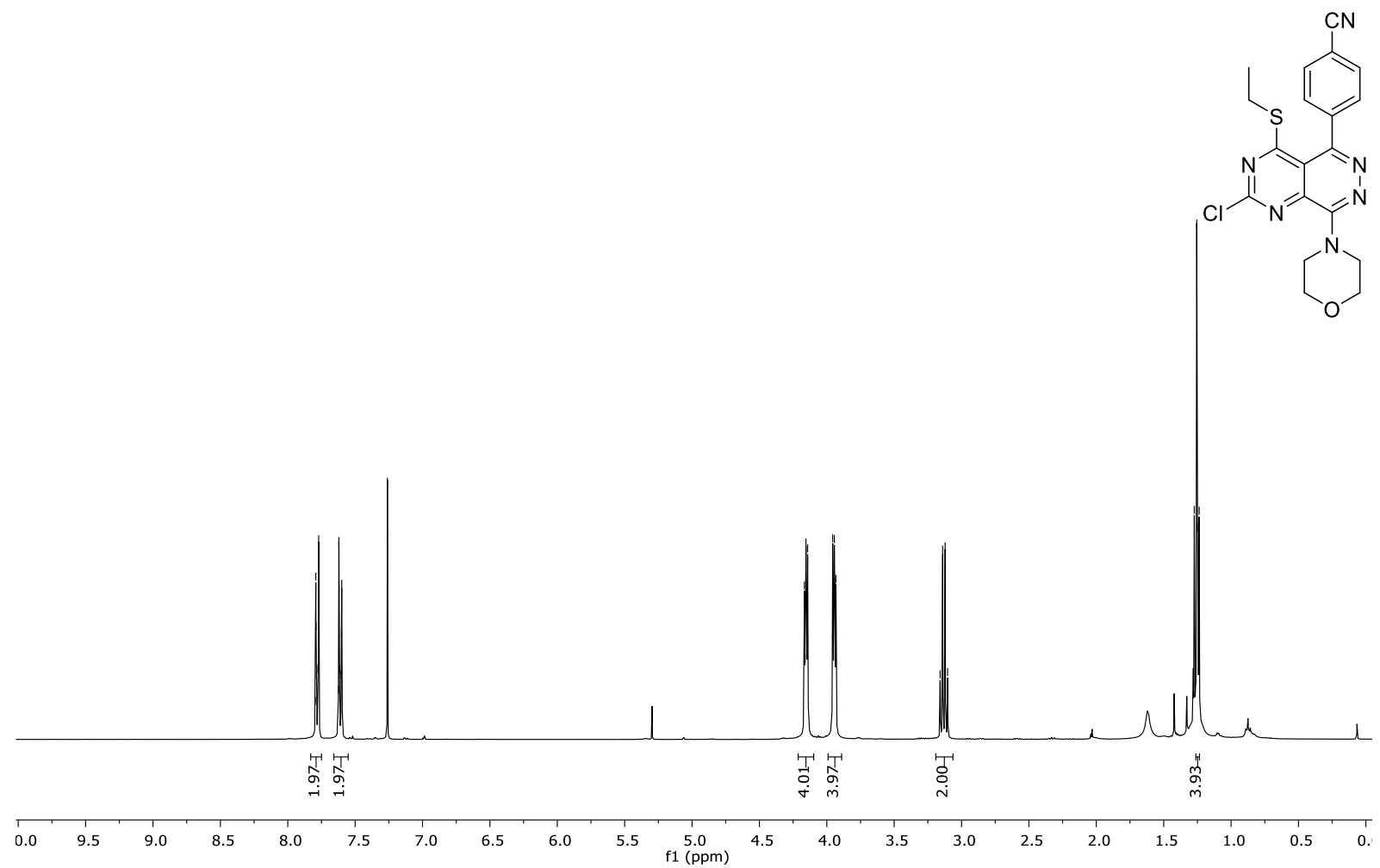

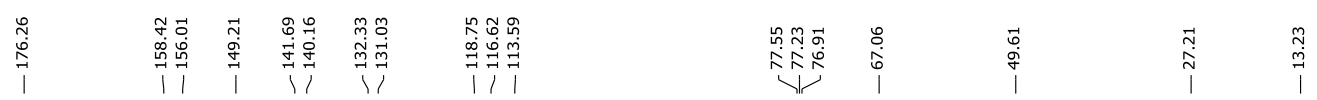

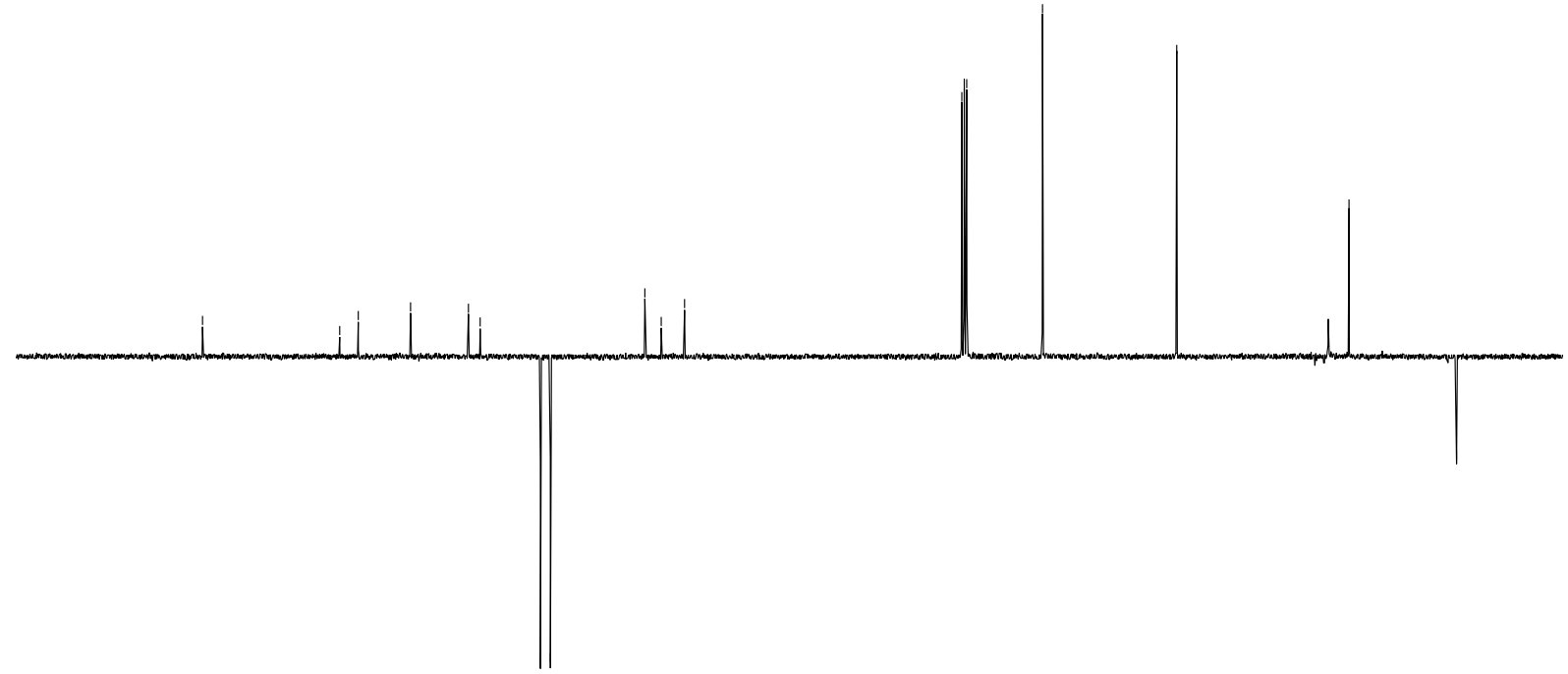

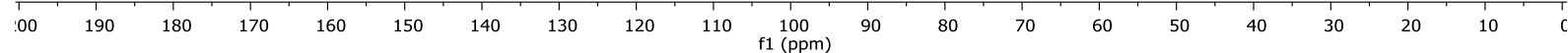


${ }^{1} \mathrm{H}$ and ${ }^{13} \mathrm{C}$ NMR spectra of $6 \mathrm{~m}$

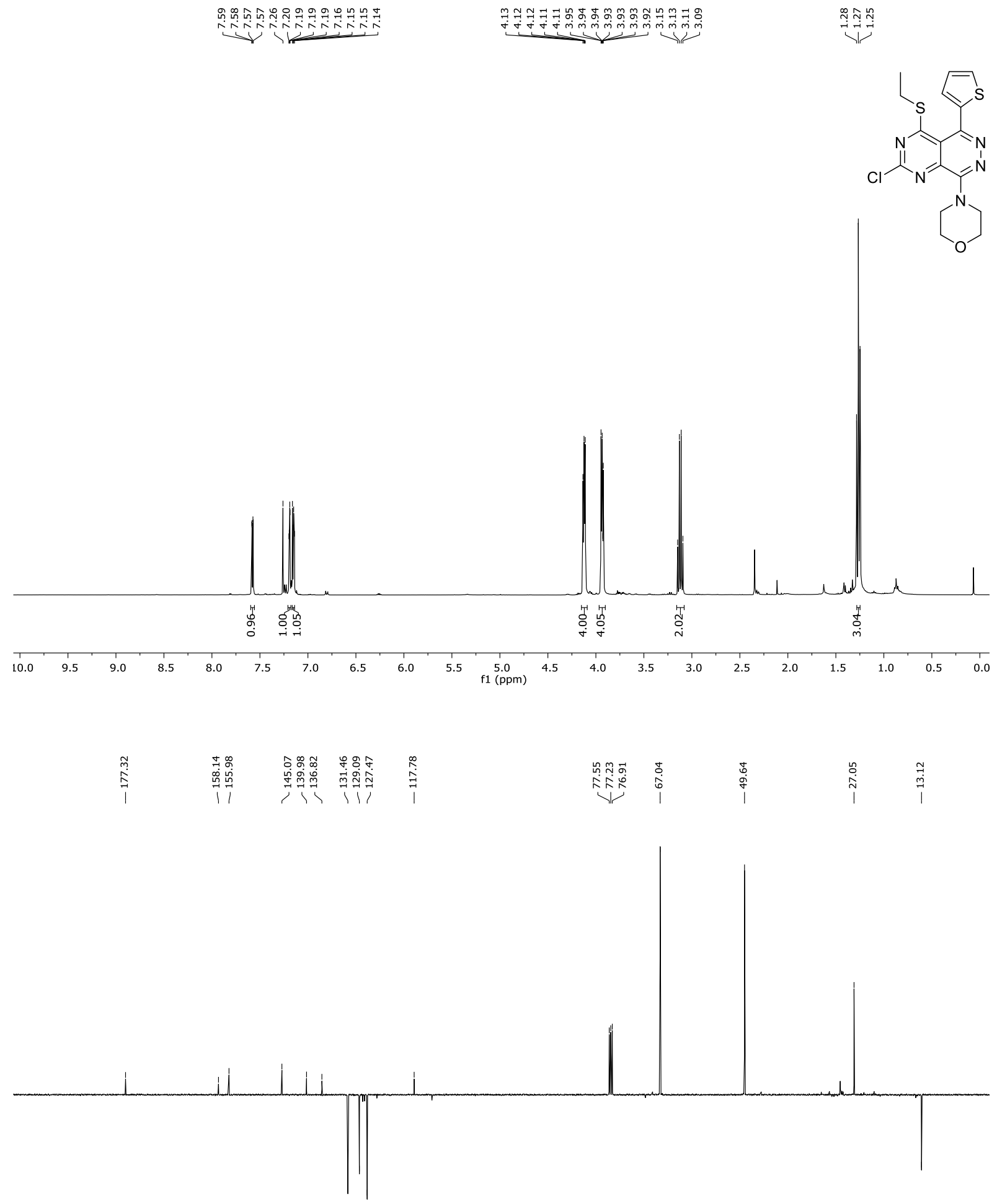

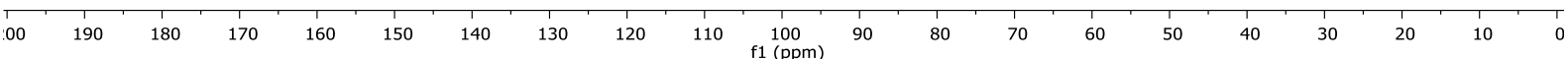


${ }^{1} \mathrm{H}$ and ${ }^{13} \mathrm{C}$ NMR spectra of $6 \mathrm{n}$

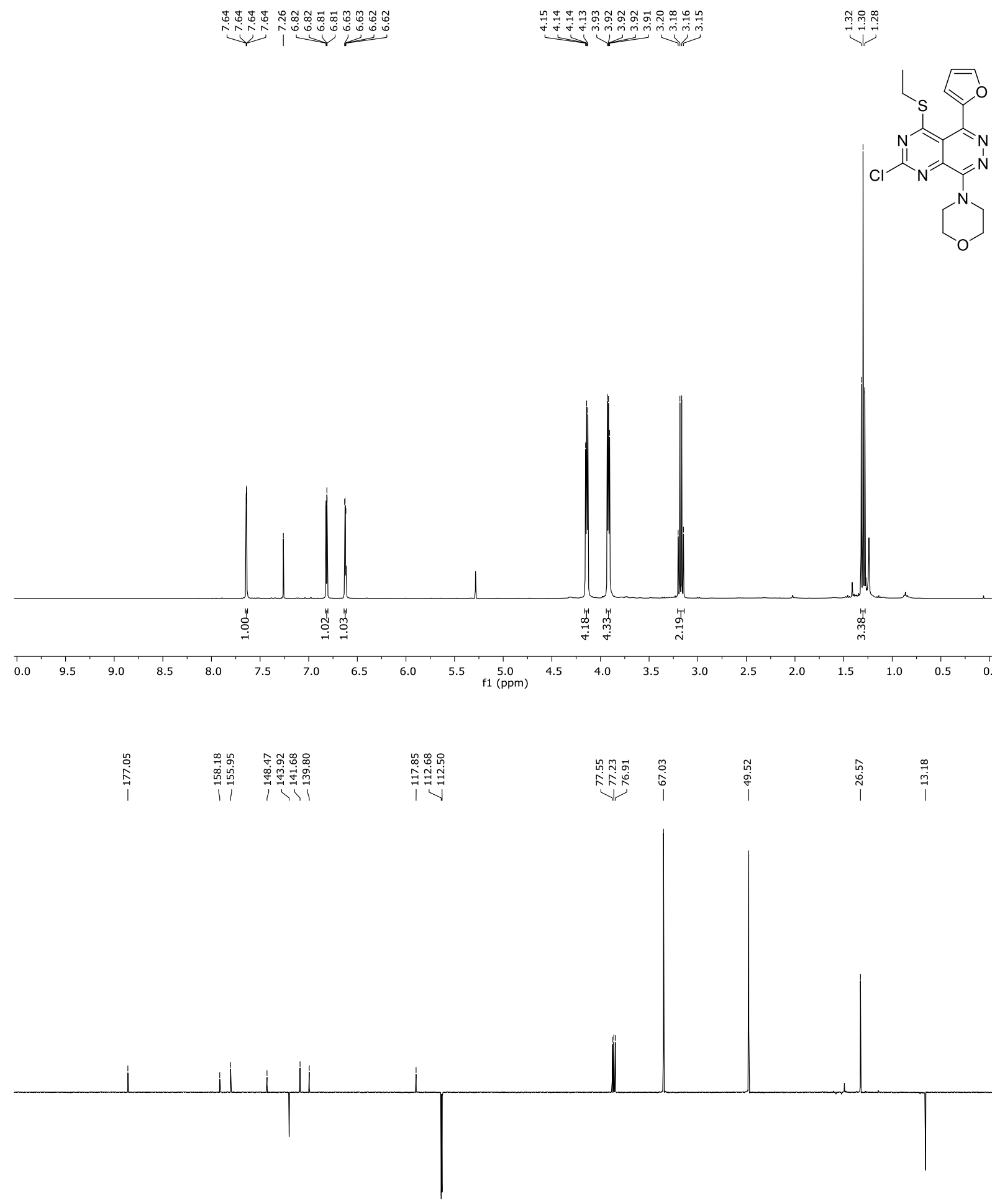

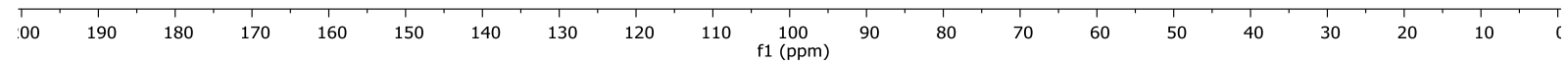


${ }^{1} \mathrm{H}$ and ${ }^{13} \mathrm{C}$ NMR spectra of $6 p$

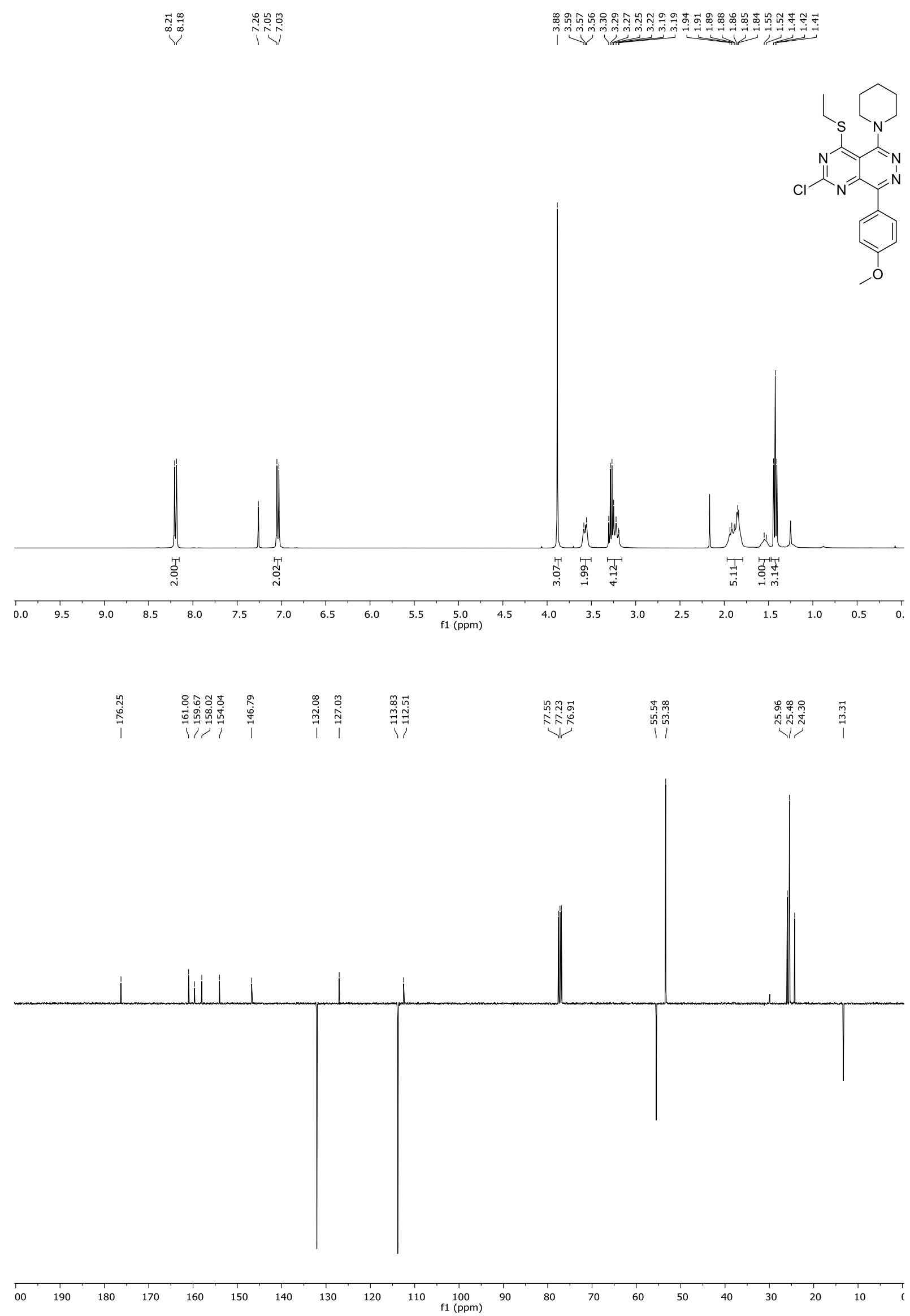




\section{${ }^{1} \mathrm{H}$ and ${ }^{13} \mathrm{C}$ NMR spectra of $\mathbf{6 q}$}

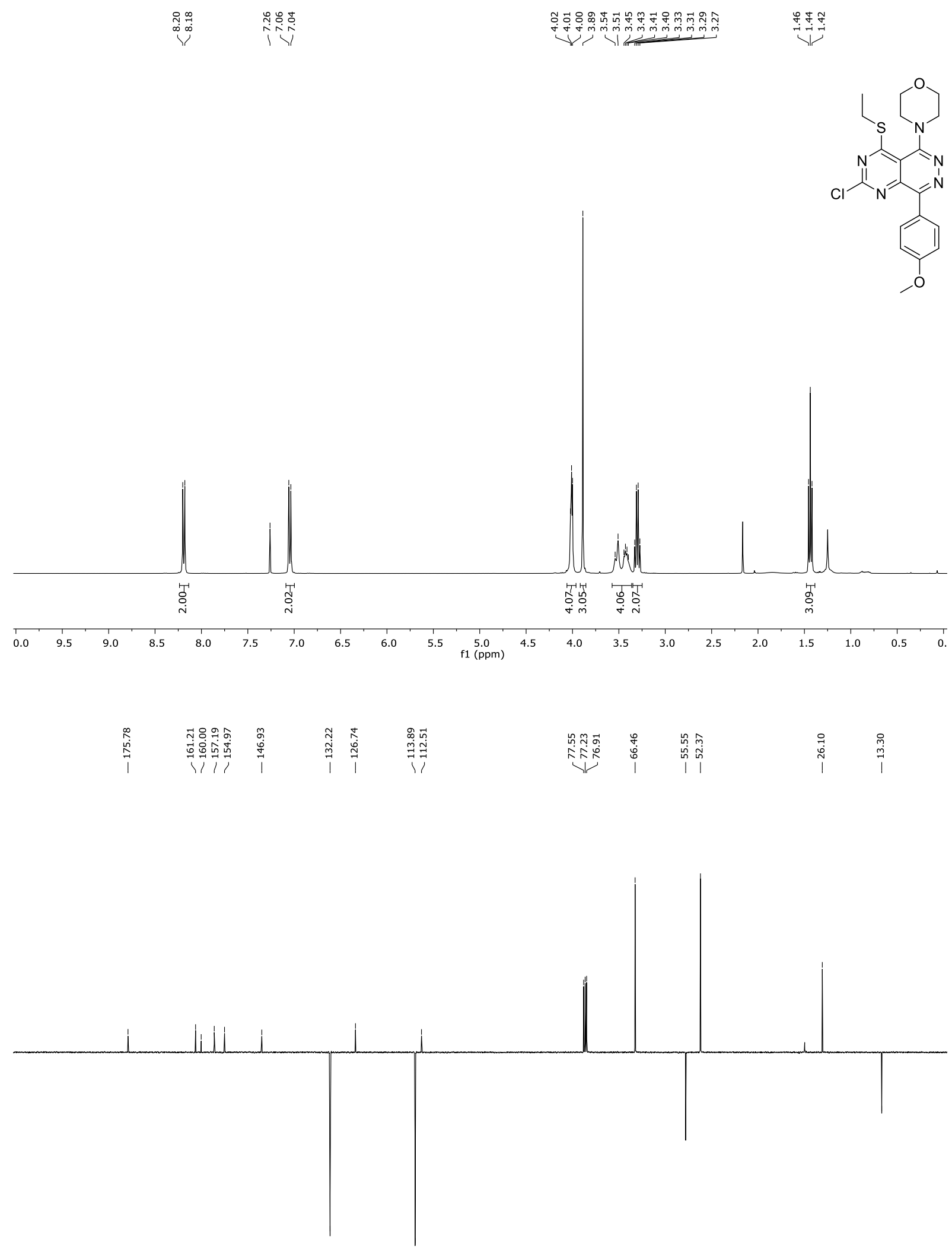

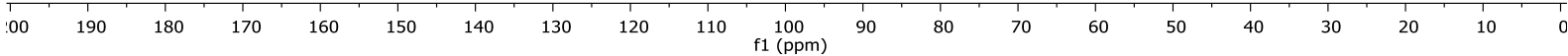


${ }^{1} \mathrm{H}$ and ${ }^{13} \mathrm{C}$ NMR spectra of $6 \mathrm{r}$

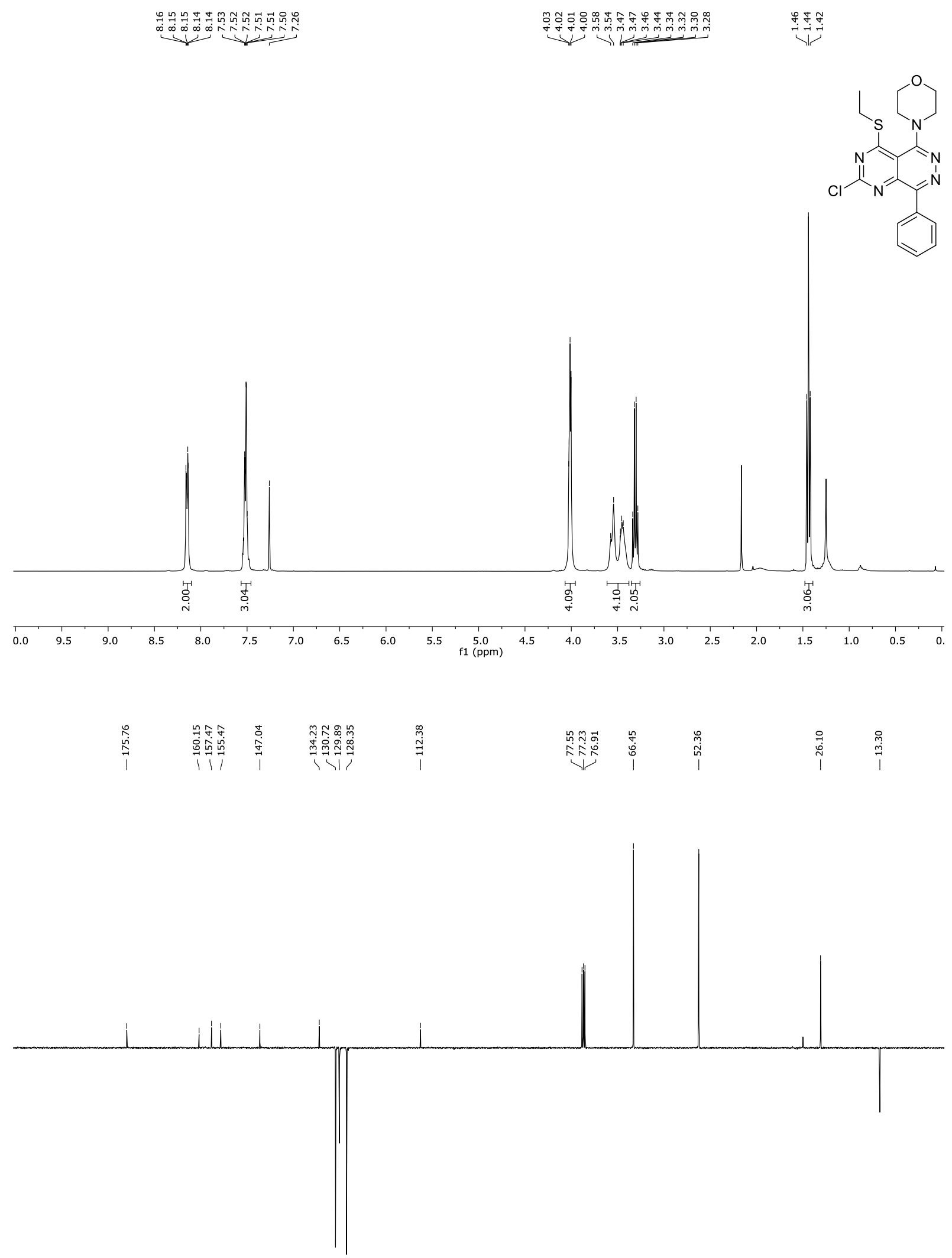

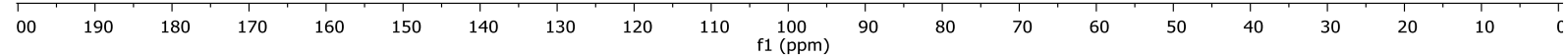


${ }^{1} \mathrm{H}$ and ${ }^{13} \mathrm{C}$ NMR spectra of $6 \mathrm{~s}$

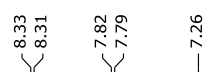

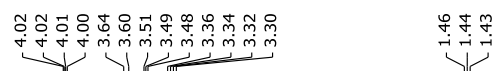

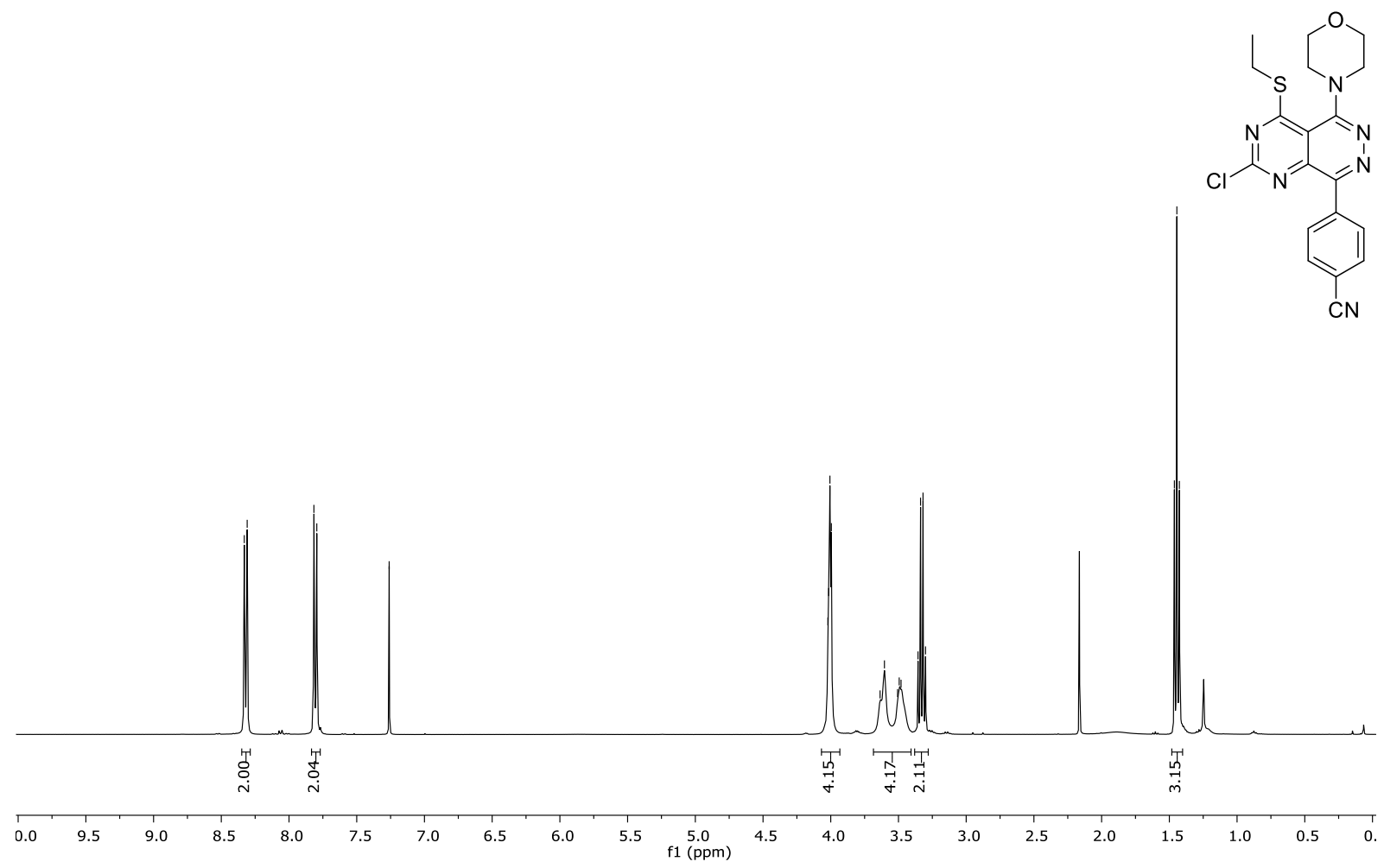

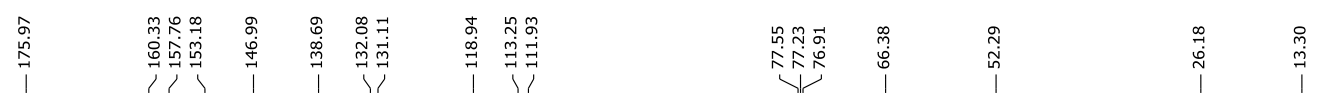

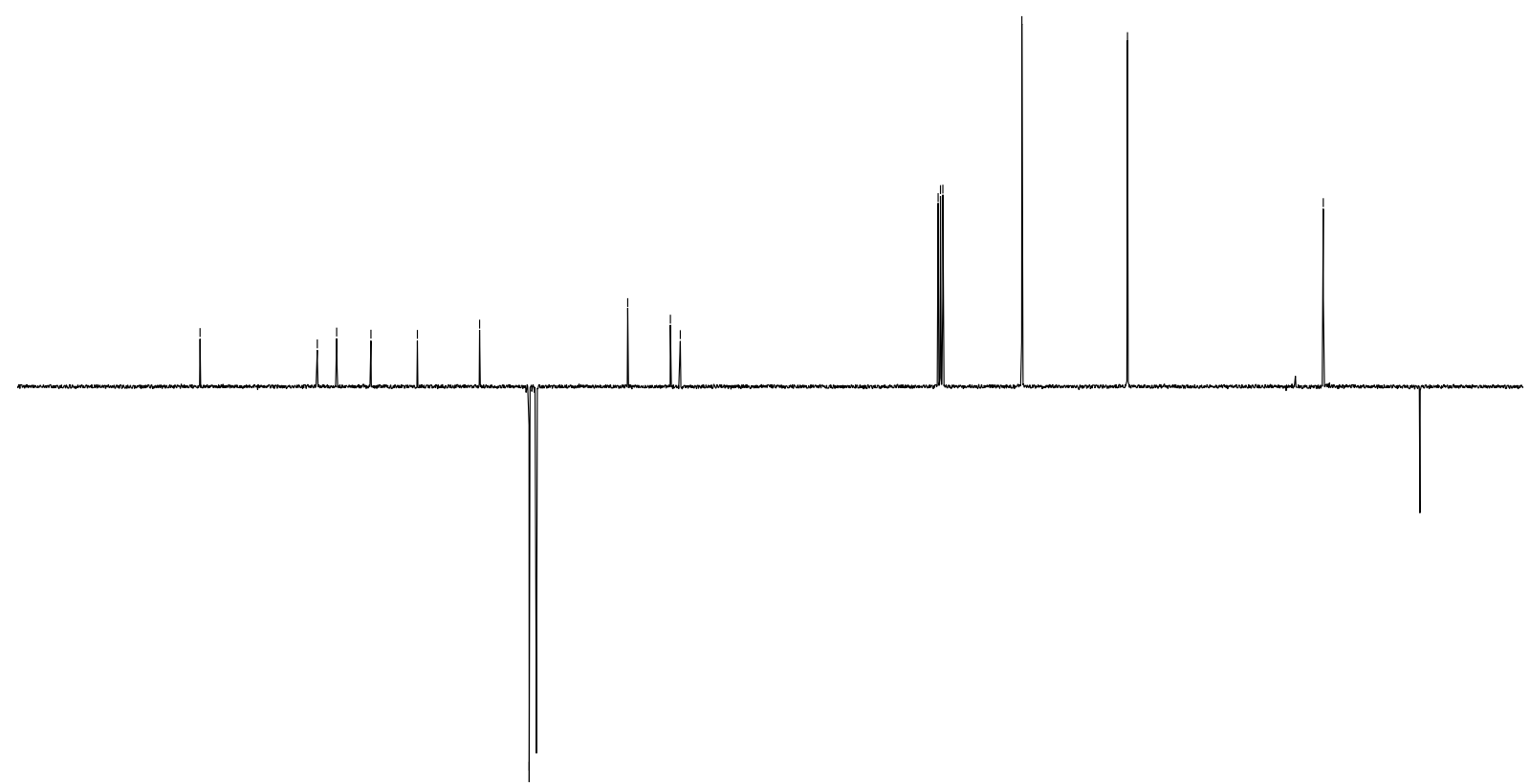

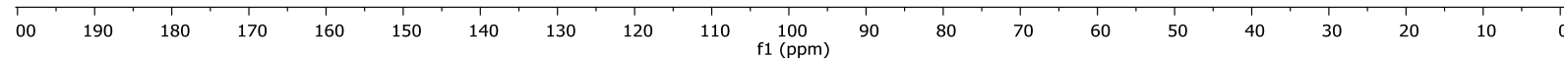


${ }^{1} \mathrm{H}$ and ${ }^{13} \mathrm{C}$ NMR spectra of $6 \mathrm{t}$

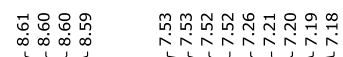

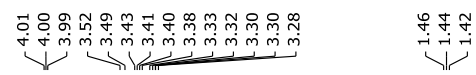

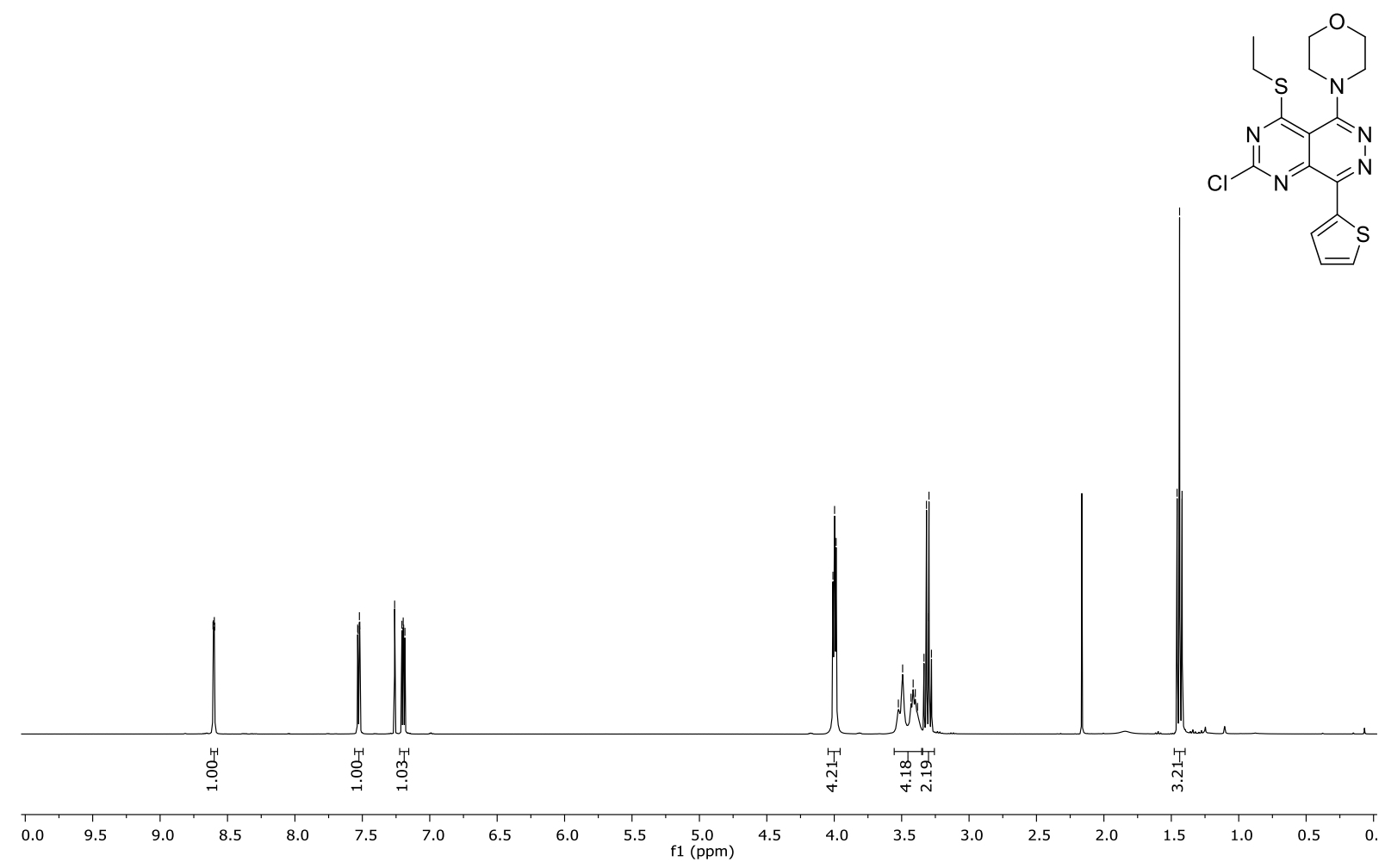

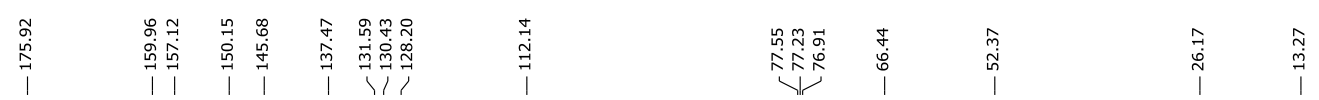

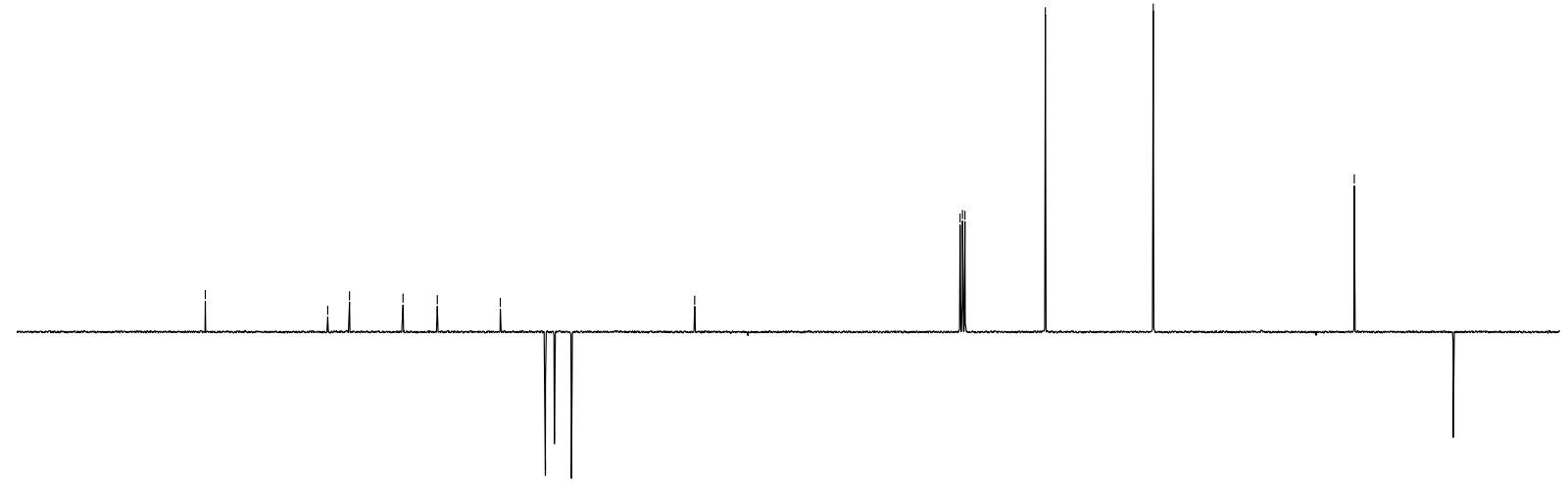

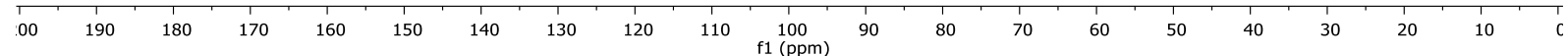


${ }^{1} \mathrm{H}$ and ${ }^{13} \mathrm{C}$ NMR spectra of $6 \mathrm{u}$

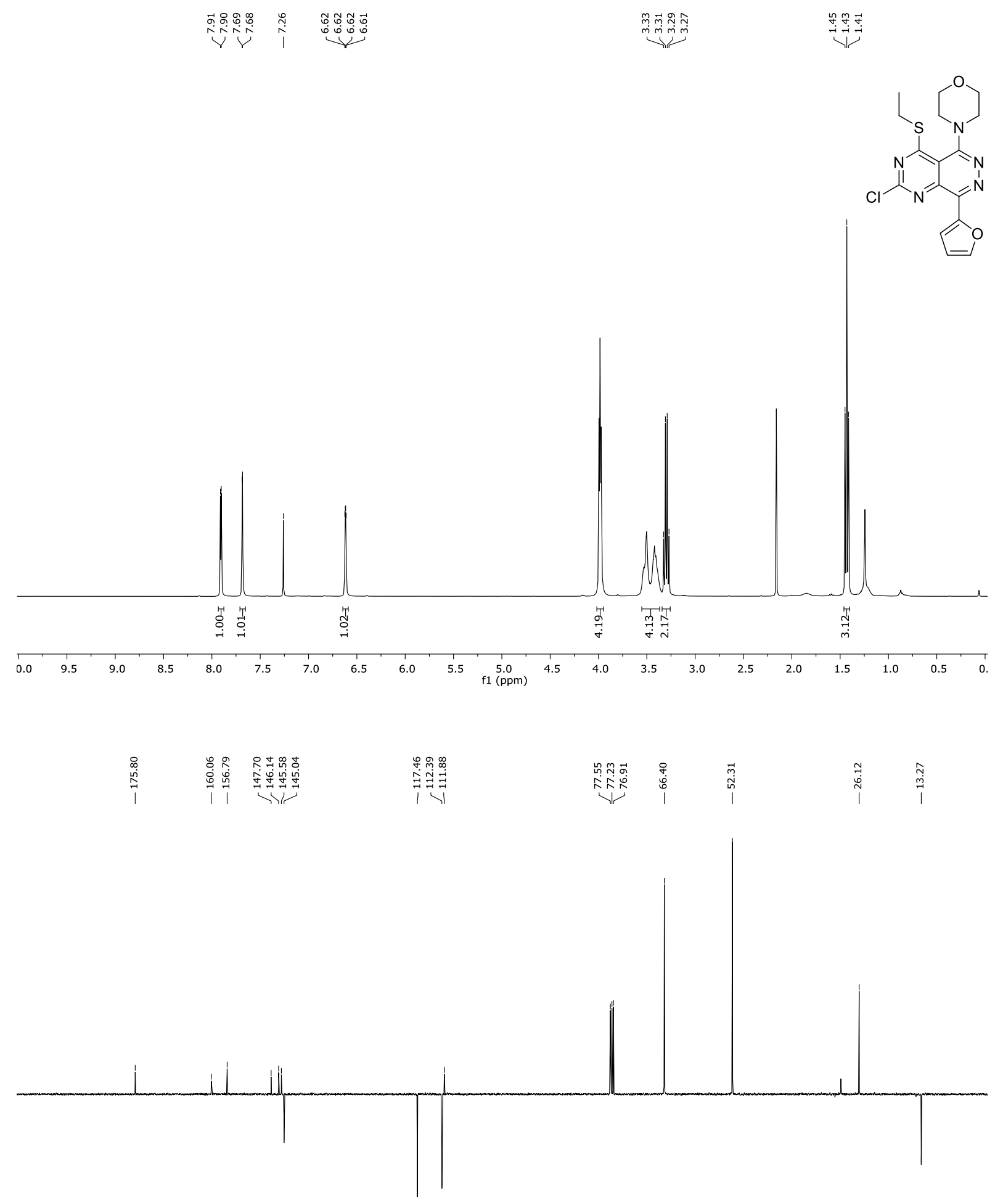

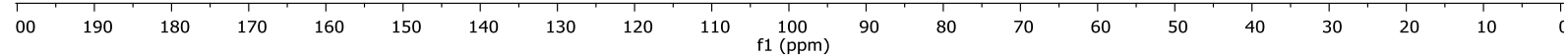


${ }^{1} \mathrm{H}$ and ${ }^{13} \mathrm{C}$ NMR spectra of 7

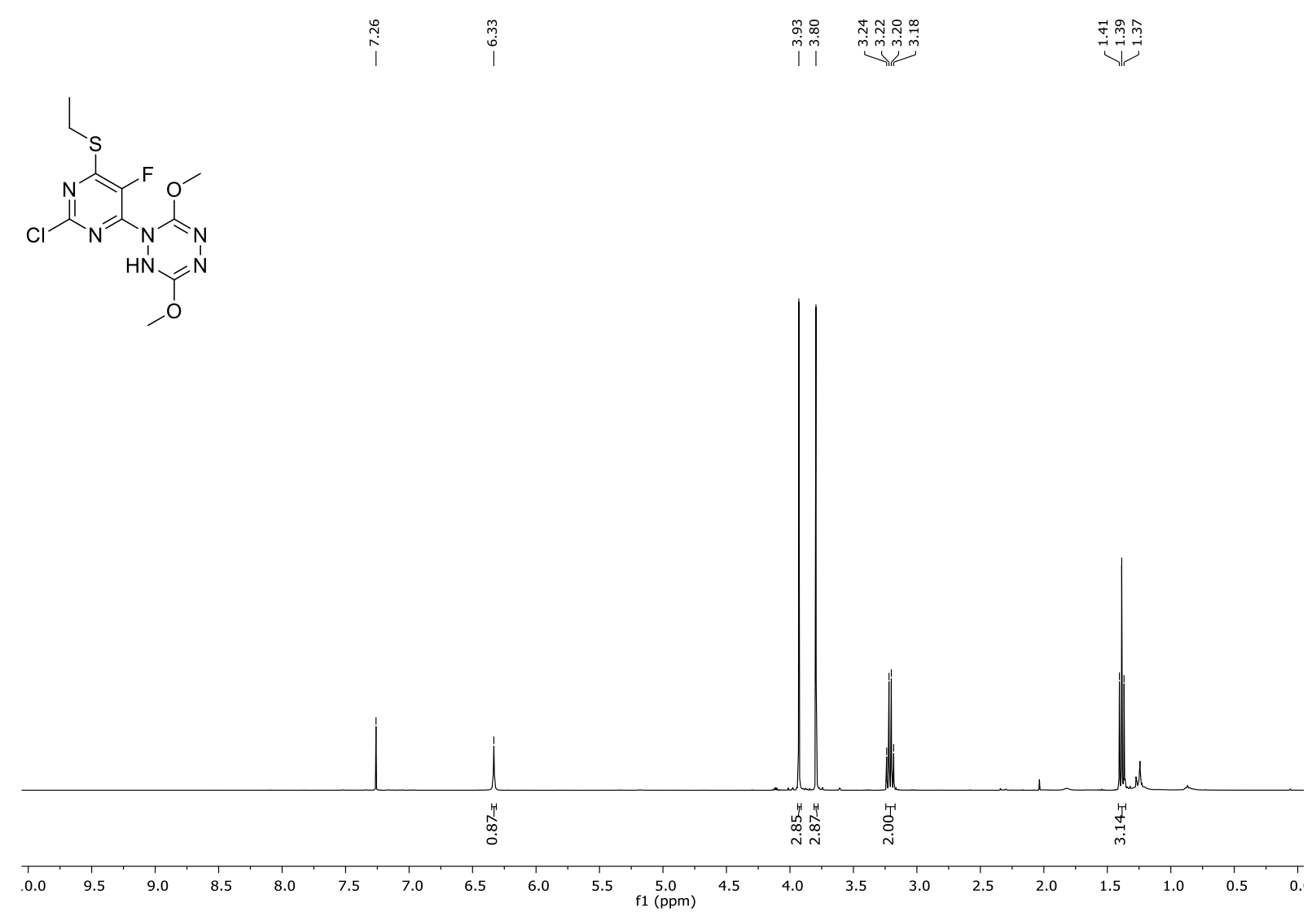

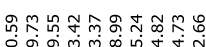

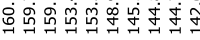

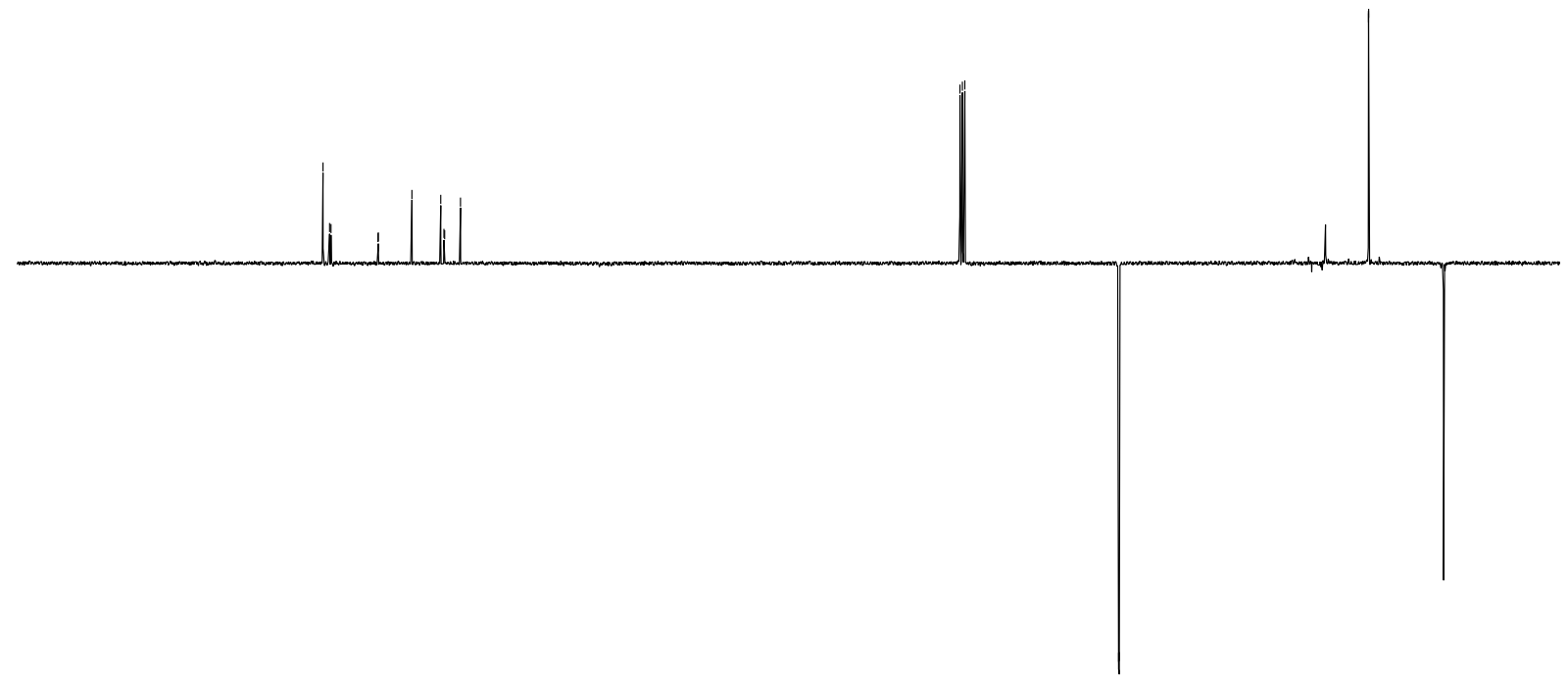

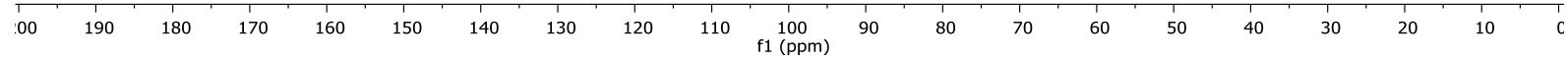


${ }^{1} \mathrm{H}$ and ${ }^{13} \mathrm{C}$ NMR spectra of 8
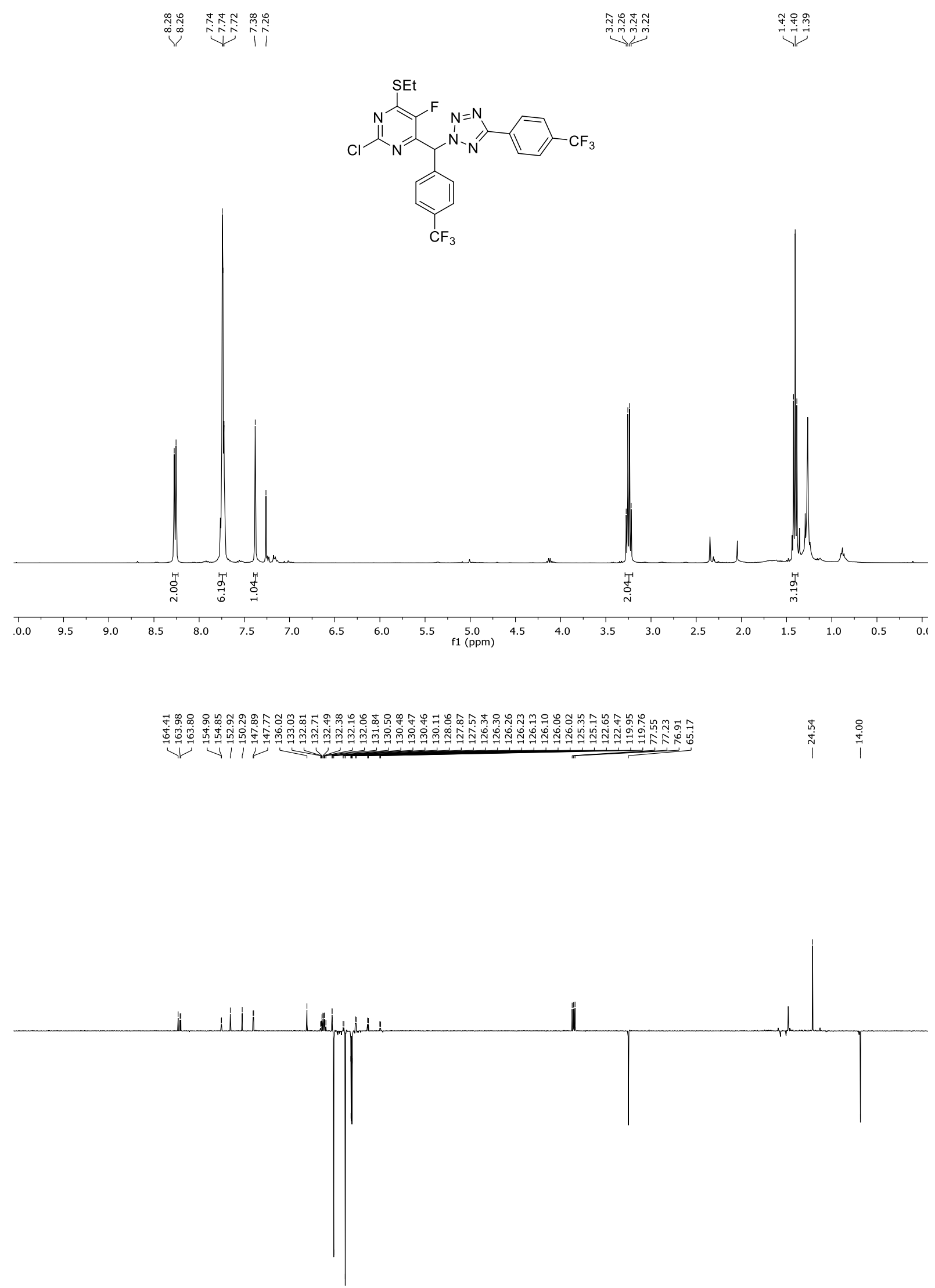

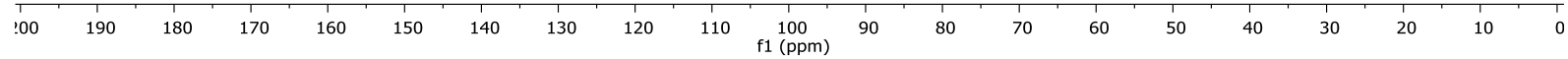


${ }^{1} \mathrm{H}$ and ${ }^{13} \mathrm{C}$ NMR spectra of 9

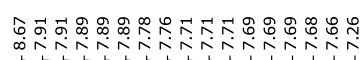

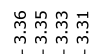

نـ<smiles>CCSc1nc(Cl)nc(C(=N/N=C/c2ccc(C(F)(F)F)cc2)c2ccc(C(F)(F)F)cc2)c1F</smiles>

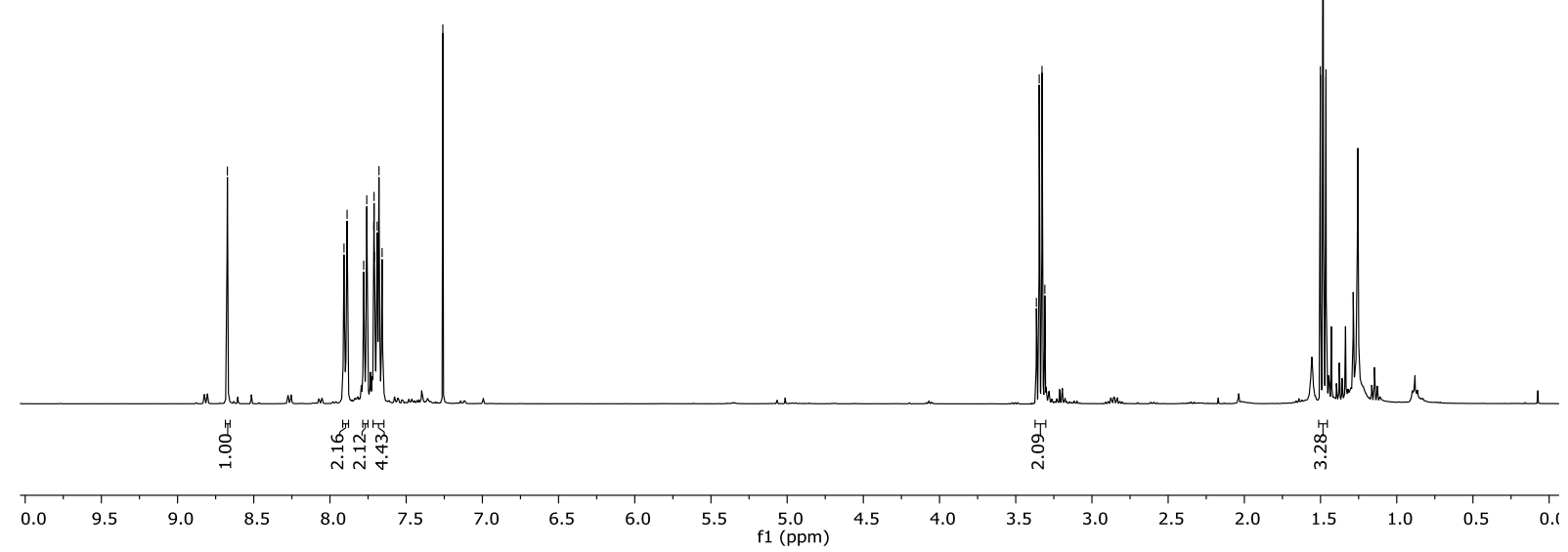

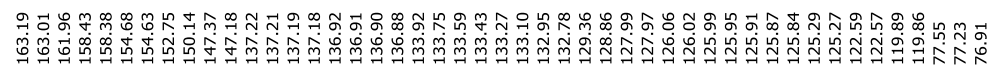

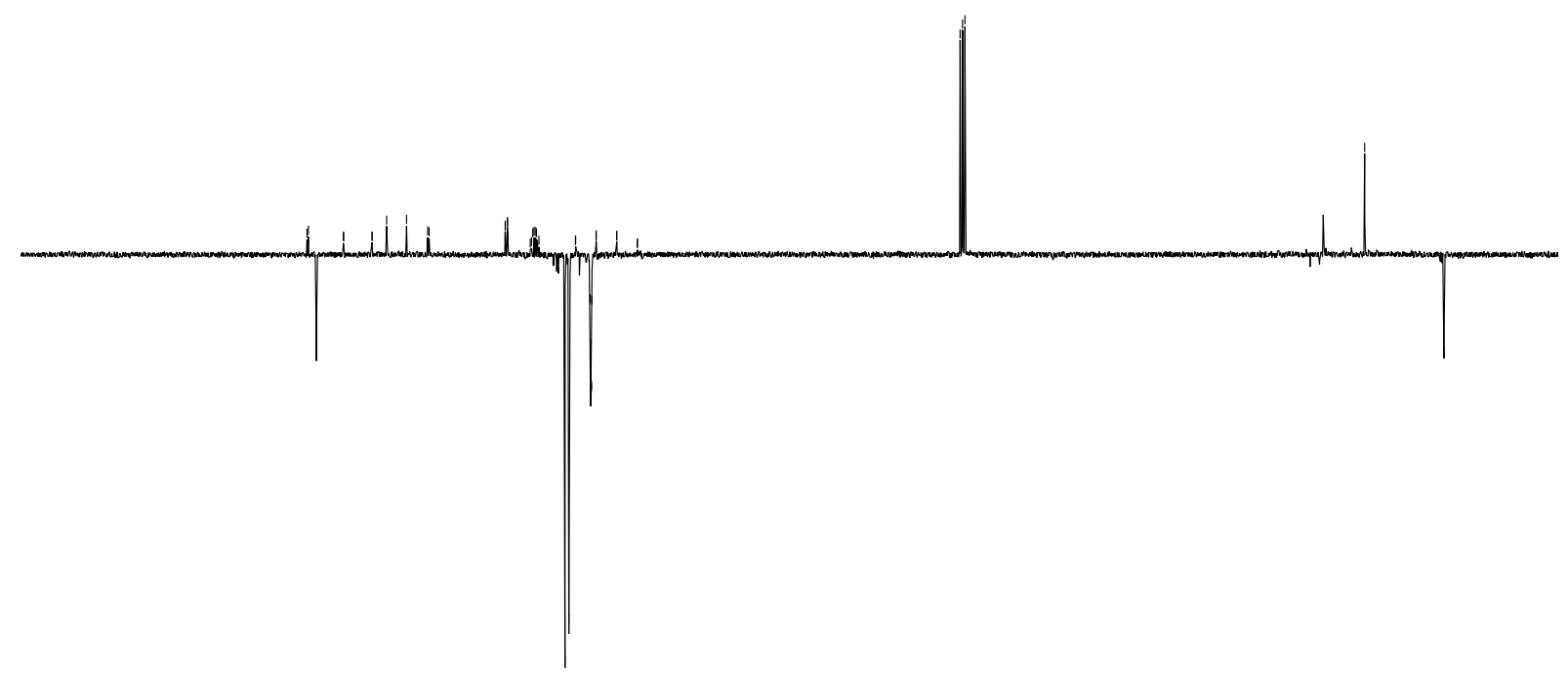

$\begin{array}{lllllllllllllllllllllllllll}1 & 100 & 190 & 180 & 170 & 160 & 150 & 140 & 130 & 120 & 110 & \begin{array}{c}100 \\ \mathrm{f} 1(\mathrm{ppm})\end{array} & 90 & 80 & 70 & 60 & 50 & 40 & 30 & 20 & 10 & 0\end{array}$ 
${ }^{1} \mathrm{H}$ and ${ }^{13} \mathrm{C}$ NMR spectra of $\mathbf{1 1}$

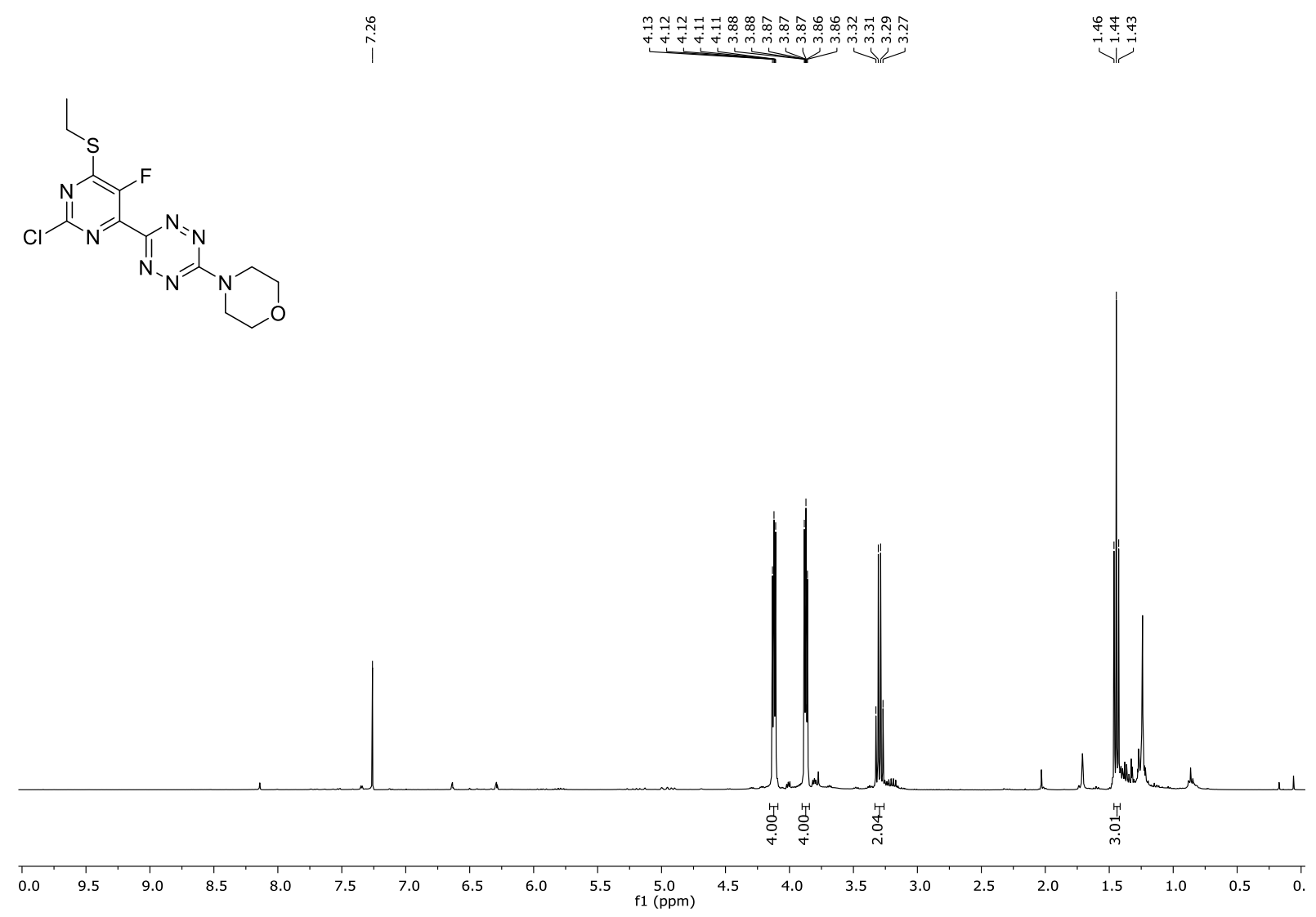

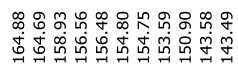

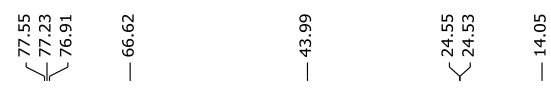

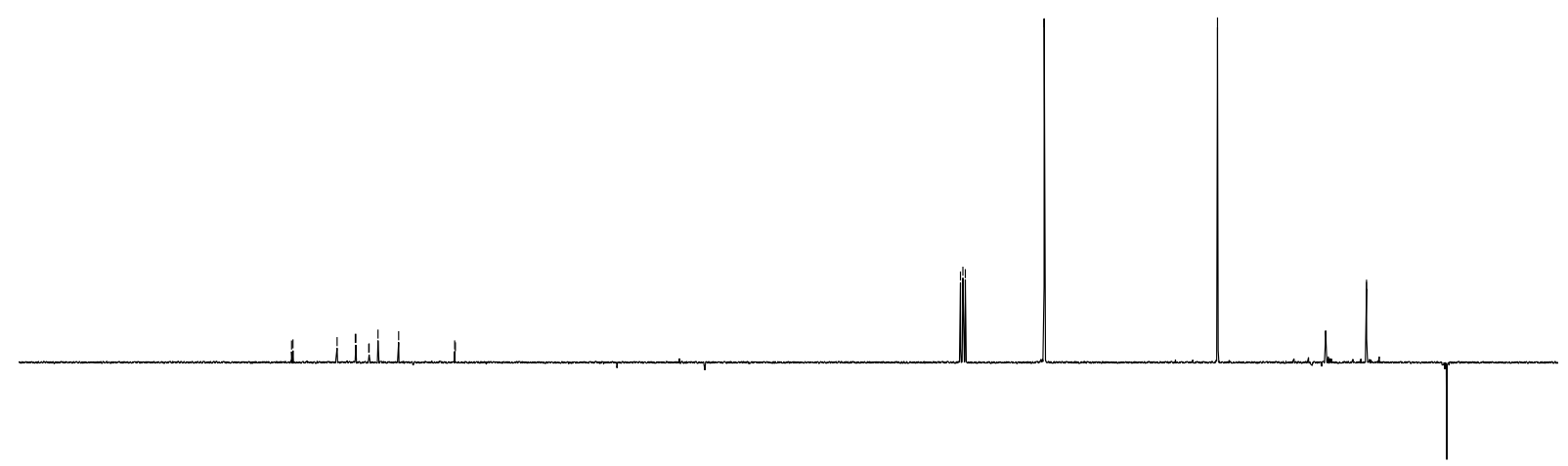

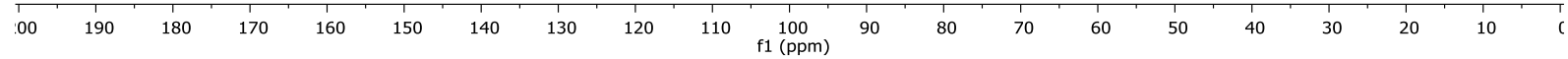




\section{${ }^{1} \mathrm{H}$ and ${ }^{13} \mathrm{C}$ NMR spectra of $\mathbf{1 2}$}

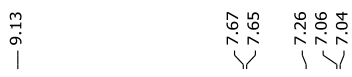

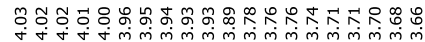

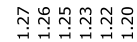

$\hat{1}$

$\longrightarrow$
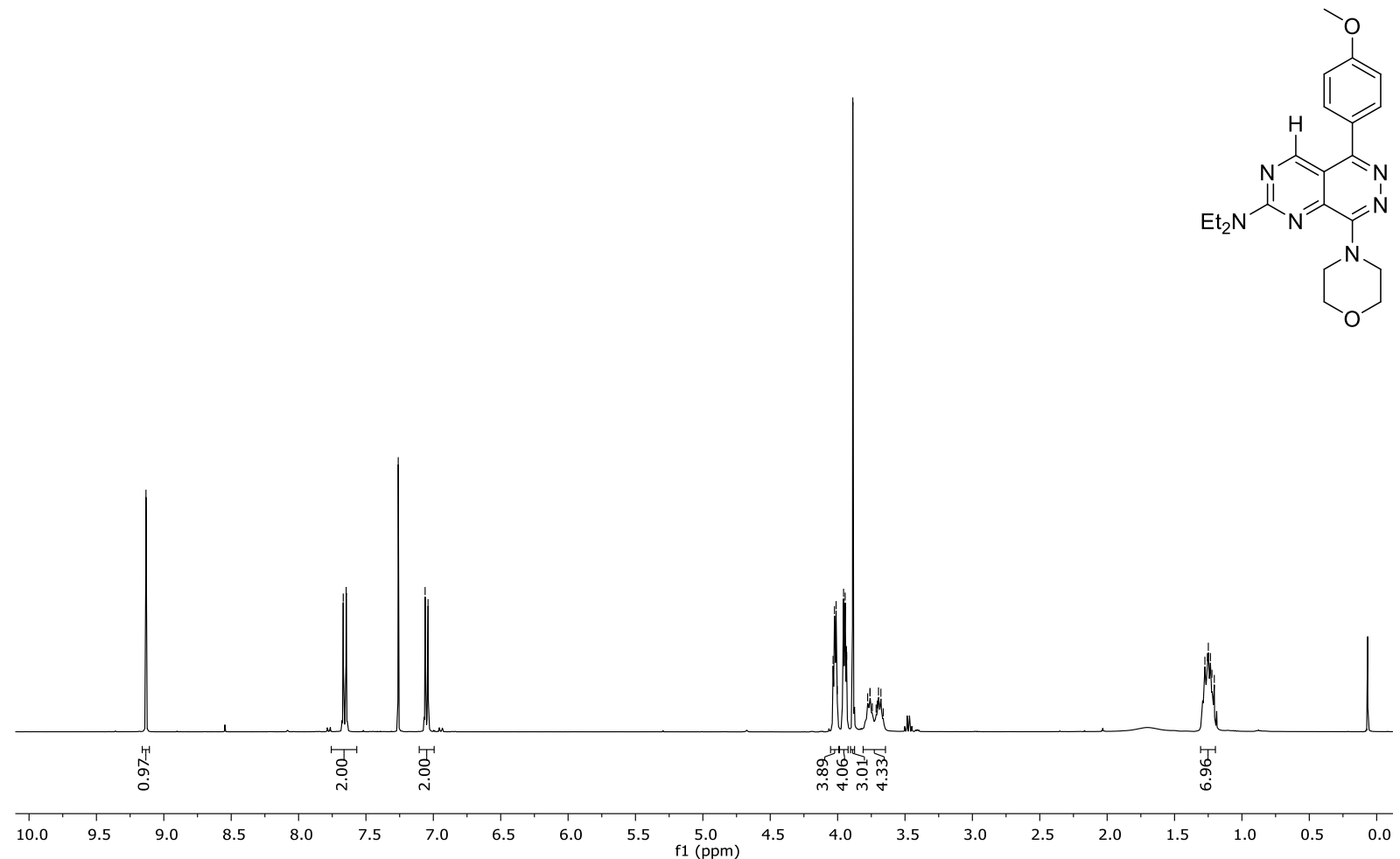

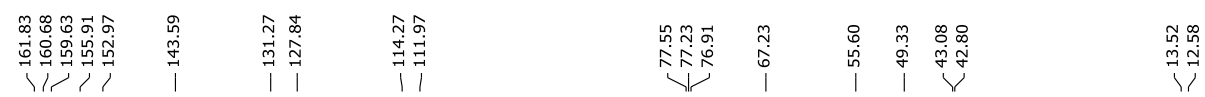

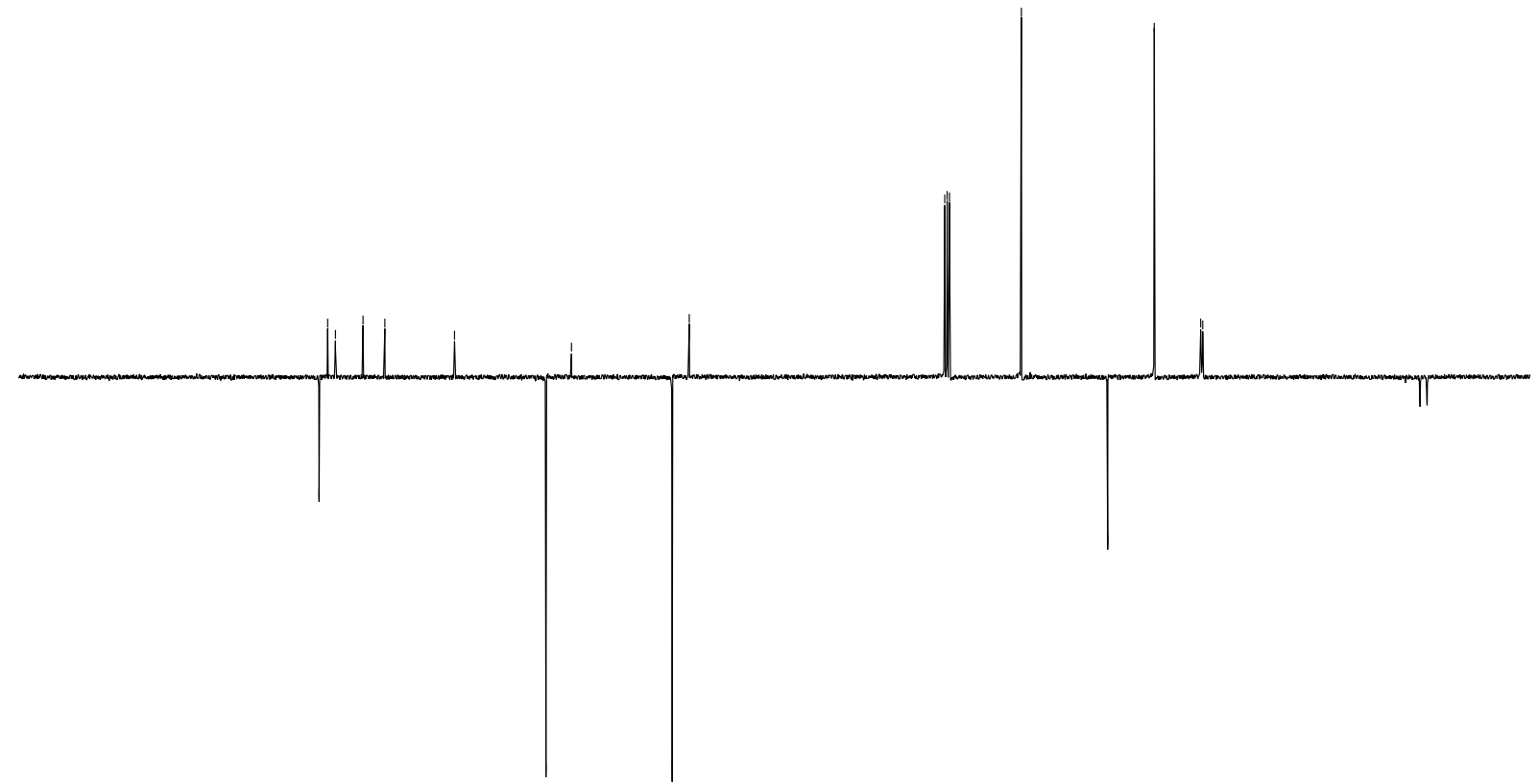

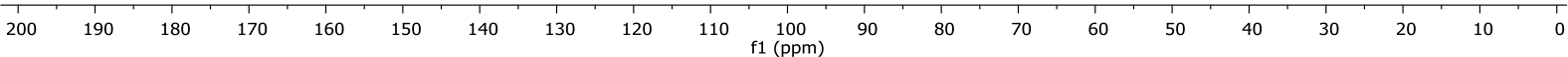




\section{${ }^{1} \mathrm{H}$ and ${ }^{13} \mathrm{C}$ NMR spectra of $\mathbf{1 3}$}

F.
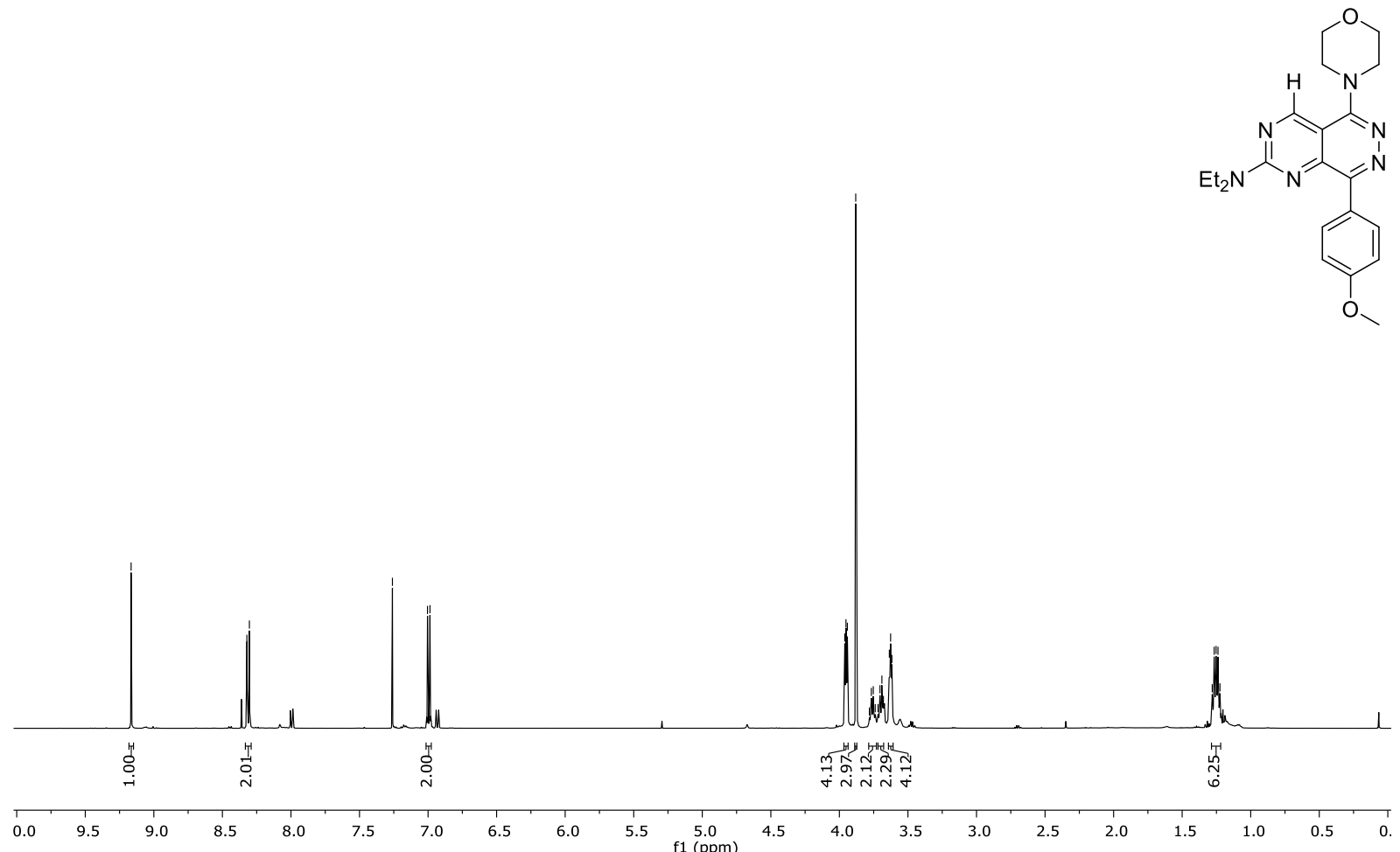

作

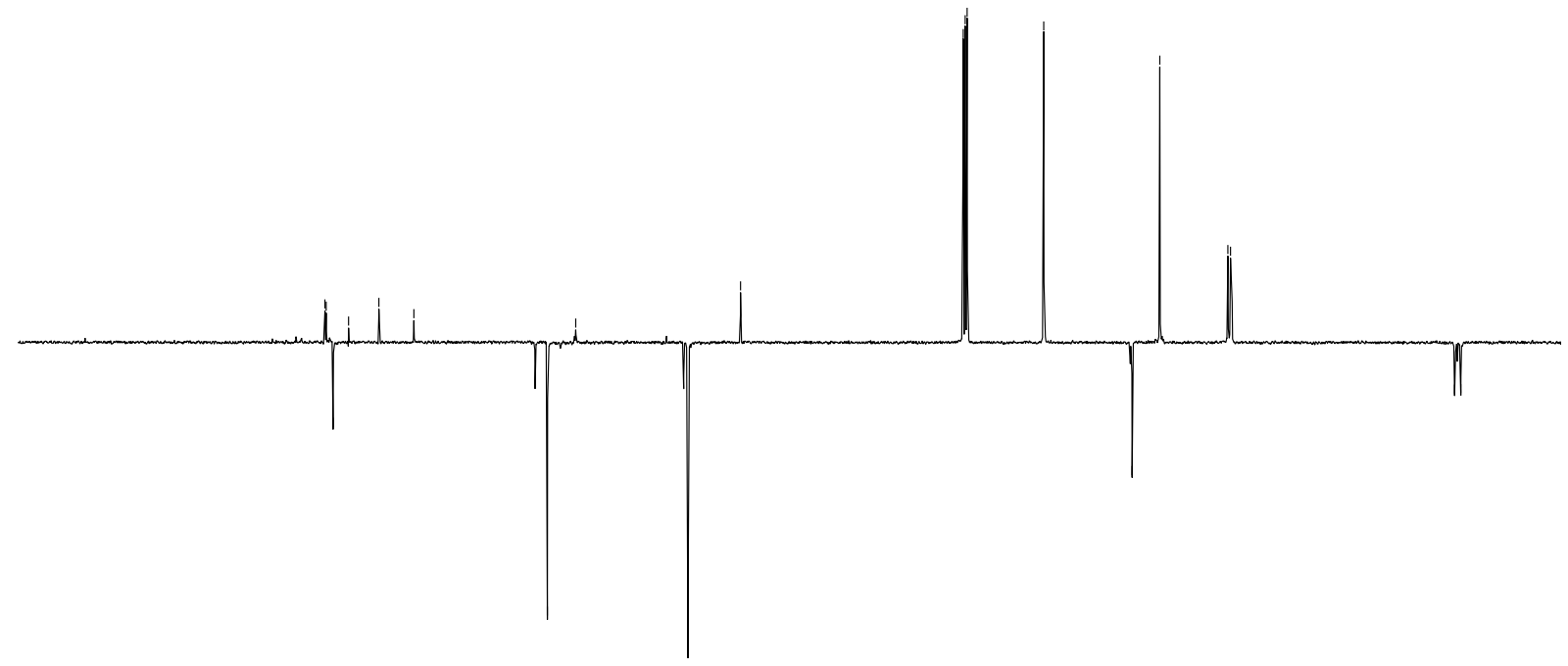

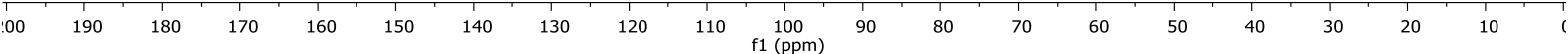

